\title{
Radioactive Materials Released from Nuclear Power Plants
}

Whepe in?

Annual Report 1981

Prepared by J. Tichler, C. Benkovitz

Brookhaven National Laboratory

Prepared for

U.S. Nuclear Regulatory

Commission 
This report was prepared as an account of work sponsored by an agency of the United States Government. Neither the United States Government nor any agency thereof, or any of their employees, makes any warranty, expressed or implied, or assumes any legal liability of responsibility for any third party's use, or the results of such use, of any information, apparatus, product or process disclosed in this report, or represents that its use by such third party would not infringe privately owned rights

\section{NOTICE}

Availability of Reference Materials Cited in NRC Publications

Most documents cited in NRC publications will be available from one of the following sources:

1. The NRC Public Document Room, 1717 H Street, N.W. Washington, DC 20555

2. The NRC/GPO Sales Program, U.S. Nuclear Regulatory Commission, Washington, DC 20555

3. The National Technical Information Service, Springfield, VA 22161

Although the listing that follows represents the majority of documents cited in NRC publications, it is not intended to be exhaustive.

Referenced documents available for inspection and copying for a fee from the NRC Public Document Room include NRC correspondence and internal NRC memoranda; NRC Office of Inspection and Enforcement bulletins, circulars, information notices, inspection and investigation notices; Licensee Event Reports; vendor reports and correspondence; Commission papers; and applicant and licensee documents and correspondence.

The following documents in the NUREG series are available for purchase from the NRC/GPO Sales Program: formal NRC staff and contractor reports, NRC-sponsored conference proceedings, and NRC booklets and brochures. Also available are Regulatory Guides, NRC regulations in the Code of Federal Regulations, and Nuclear Regulatory Commission /ssuances

Documents available from the National Technical Information Service include NUREG series reports and technical reports prepared by other federal agencies and reports prepared by the Atomic Energy Commission, forerunner agency to the Nuclear Regulatory Commission.

Documents available from public and special technical libraries include all open literature items, such as books, journal and periodical articles, and transactions. Federal Register notices, federal and state legislation, and congressional reports can usually be obtained from these libraries.

Documents such as theses, dissertations, foreign reports and translations, and non-NRC conference proceedings are available for purchase from the organization sponsoring the publication cited.

Single copies of NRC draft reports are available free, to the extent of supply, upon written request to the Division of Technical Information and Document Control, U.S. Nuclear Regulatory Commission, Washington, DC 20555.

Copies of industry codes and standards used in a substantive manner in the NRC regulatory process are maintained at the NRC Library, 7920 Norfolk Avenue, Bethesda, Maryland, and are available there for reference use by the public. Codes and standards are usually copyrighted and may be purchased from the originating organization or, if they are American National Standards, from the American National Standards Institute, 1430 Broadway, New York, NY 10018 


\section{DISCLAIMER}

This report was prepared as an account of work sponsored by an agency of the United States Government. Neither the United States Government nor any agency Thereof, nor any of their employees, makes any warranty, express or implied, or assumes any legal liability or responsibility for the accuracy, completeness, or usefulness of any information, apparatus, product, or process disclosed, or represents that its use would not infringe privately owned rights. Reference herein to any specific commercial product, process, or service by trade name, trademark, manufacturer, or otherwise does not necessarily constitute or imply its endorsement, recommendation, or favoring by the United States Government or any agency thereof. The views and opinions of authors expressed herein do not necessarily state or reflect those of the United States Government or any agency thereof. 


\section{DISCLAIMER}

Portions of this document may be illegible in electronic image products. Images are produced from the best available original document. 


\section{Radioactive Materials Released from Nuclear Power Plants}

\section{Annual Report 1981}

Manuscript Completed: May 1984

Date Published: June 1984

Prepared by

J. Tichler, C. Benkovitz

Brookhaven National Laboratory

Upton, NY 11973

\section{Prepared for \\ Division of Budget and Analysis \\ Office of Resource Management \\ U.S. Nuclear Regulatory Commission \\ Washington, D.C. 20555 \\ NRC FIN A3188}

\section{DISCLAIMER}

This report was prepared as an account of work sponsored by an agency of the United States Government. Neither the United States Government nor any agency thereof, nor any of their employees, makes any warranty, express or implied, or assumes any legal liability or responsibility for the accuracy, completeness, or usefulness of any information, apparatus, product, or process disclosed, or represents that its use would not infringe privately owned rights. Reference herein to any specific commercial product, process, or service by trade name, trademark, manufacturer, or otherwise does not necessarily constitute or imply its endorsement, recommendation, or favoring by the United States Government or any agency thereof. The views and opinions of authors expressed herein do not necessarily state or reflect those of the United States Government or any agency thereof. 
1. "Report on Releases of Radioactivity in Effluents and Solid Wastes from Nuclear Power Plants for 1972," Directorate of Regulatory Operations, August 1973.

2. "Summary of Radioactivity Releases in Effluents from Nuclear Power Plants During 1973," NUREG-75/001, January 1975.

3. "Radioactive Materials Released from Nuclear Power Plants, 1974," NUREG-0077, June 1976.

4. "Radioactive Materials Released from Nuclear Power Plants, 1975," NUREG-0218, March 1977.

5. "Radioactive Materials Released from Nuclear Power Plants, 1976," NUREG-0367, March 1978.

6. "Radioactive Materials Released from Nuclear Power Plants, 1977," NUREG-0521, January 1979.

7. "Radioactive Materials Released from Nuclear Power Plants, 1978," NUREG/CR-1497, BNL-NUREG-51192, March 1981.

8. "Radioactive Materials Released from Nuclear Power Plant8, 1979," NUREG/CR-2227, BNL-NUREG-51416, November 1981.

9. "Radioactive Materials Released from Nuclear Power Plant8, 1980," NUREG/CR-2907, BNL-NUREG-51581, Vo1. 1, January 1983. 


\section{NOTICE}

This report was prepared as an account of work sponsored by an agency of the United States Government. Neither the United States Government nor any agency thereof, or any of their employees, makes any warranty, expressed or implied, or assumes any legal liability of responsibility for any third party's use, or the results of such use, of any information, apparatus, product or process disclosed in this report, or represents that its use by such third party would not infringe privately owned rights.

\section{Availability of Reference Materials Cited in NRC Publications}

Most documents cited in NRC publications will be available from one of the following sources:

1. The NRC Public Document Room, 1717 H Street, N.W. Washington, DC 20555

2. The NRC/GPO Sales Program, U.S. Nuclear Regulatory Commission, Washington, DC 20555

3. The National Technical Information Service, Springfield,. VA 22161

Although the listing that follows represents the majority of documents cited in NRC publications. it is not intended to be exhaustive.

Referenced documents available for inspection and copying for a fee from the NRC'Public Document Room include NRC correspondence and irternal NRC memoranda; NRC Office of Inspection and Enforcement bulletins, circulars, information notices, inspection and investigation notices; Licensee Event Reports; vendor reports and correspondence; Commission papers; and applicant and licensee documents and correspondence.

The following documents in the NUREG series are available for purchase from the NRC/GPO Sales Program: formal NRC staff and contractor reports, NRC-sponsored conference proceedings, and NRC booklets and brochures. Also available are Regulatory Guides, NRC regulations in the Code of Federal Regulations, and Nuclear Regulatory Commission Issuances.

Documents available from the National Technical Information Service include NUREG series reports and technical reports prepared by other federal agencies and reports prepared by the Atomic Energy Commission, forerunner agency to the Nuclear Regulatory Commission.

Documents available from public and special technical libraries include all open literature items, such as books, journal and periodical articles, and transactions. Federal Register notices, federal and state legislation, and congressional reports can usually be obtained from these libraries.

Documents such as theses, dissertations, foreıgn reports and translations, and non-NRC conference proceedings are available for purchase trom the organization sponsoring the publication cited.

Single copies of NRC draft reports are available free upon written request to the Division of Tech. nical Information and Document Control, U.S. Nuclear Regulatory Commission. Washington, DC 20555.

Copies of industry codes and standards used in a substantive manner in the NRC regulatory process are maintained at the NRC Library. 7920 Norfolk Avenue, Bethesda, Maryland, and are available there for reference use by the public. Codes and standards are usually copyrighted and may be purchased from the originating organization or, if they are American National Standards, from the American National Standards Institute. 1430 Broadway, New York, NY 10018. 
0

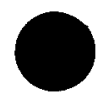


Releases of radioactive materials in alrborne and liquid effluents from commercial light water reactors during 1981 have been complled and reported. Data on solid waste shipments as well as selected operating information have been included. Th1s report supplements earlier annual reports issued by the former Atomic Energy Commission and the Nuclear Regulatory Commission. The 1981 release data are summarized in tabular form. Data covering specific radionuclides are summarized. 


$$
\text { , }
$$




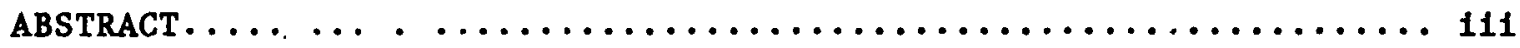

1.0 Introduction. $\ldots \ldots \ldots \ldots \ldots \ldots \ldots \ldots \ldots \ldots \ldots \ldots \ldots \ldots \ldots \ldots \ldots$

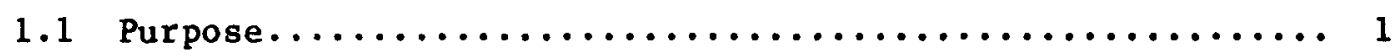

1.2 scope..................................... 1

1.3 Source of Data.............................. 1

2.0 Tabulated Data.............................. 2

2.1 Airborne and Liquid Effluent................... 2

2.2 Solid Waste............................... 2

2.3 Energy Generation............................. 2

2.4 Individual Plant Summaries..................... 2

2.5 Numerical Notation.......................... 3

3.0 summary $\ldots \ldots \ldots \ldots \ldots \ldots \ldots \ldots \ldots \ldots \ldots \ldots \ldots \ldots \ldots \ldots \ldots \ldots \ldots \ldots \ldots$

4.0 List of Tables............................. 4

Appendix A - Individual Plant Summaries

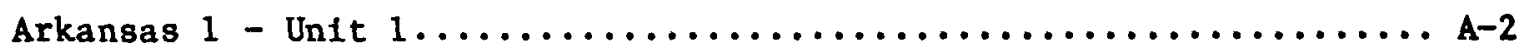

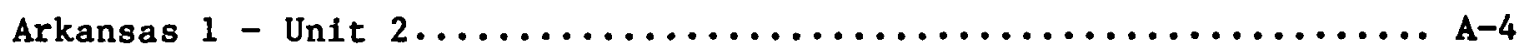

Beaver valley $1 \ldots \ldots \ldots \ldots \ldots \ldots \ldots \ldots \ldots \ldots \ldots \ldots \ldots \ldots \ldots \ldots \ldots$

B1g Rock Polnt $1 \ldots \ldots \ldots \ldots \ldots \ldots \ldots \ldots \ldots \ldots \ldots \ldots \ldots \ldots \ldots \ldots \ldots$

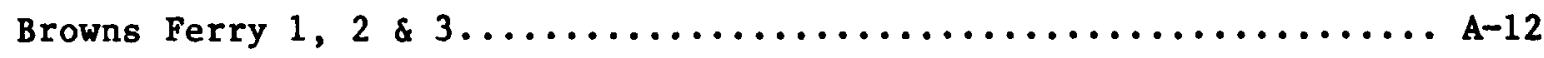

Brunswick $1 \& 2 \ldots \ldots \ldots \ldots \ldots \ldots \ldots \ldots \ldots \ldots \ldots \ldots \ldots \ldots \ldots \ldots \ldots \ldots$

Calvert C11ffs $1 \& 2 \ldots \ldots \ldots \ldots \ldots \ldots \ldots \ldots \ldots \ldots \ldots \ldots \ldots \ldots \ldots \ldots \ldots \ldots$

Cook $1 \& 2 \ldots \ldots \ldots \ldots \ldots \ldots \ldots \ldots \ldots \ldots \ldots \ldots \ldots \ldots \ldots \ldots \ldots \ldots \ldots \ldots \ldots \ldots \ldots \ldots-22$

Cooper.......................................... 


\section{Page}

Crystal River $3 . \ldots \ldots \ldots \ldots \ldots \ldots \ldots \ldots \ldots \ldots \ldots \ldots \ldots \ldots \ldots \ldots \ldots \ldots \ldots$

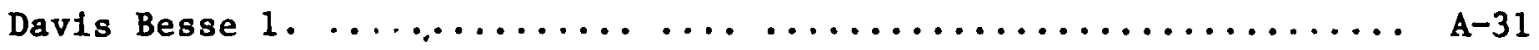

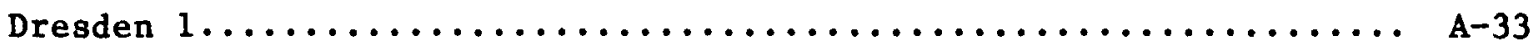

Dresden $2 \& 3 \ldots \ldots \ldots \ldots \ldots \ldots \ldots \ldots \ldots \ldots \ldots \ldots \ldots \ldots \ldots \ldots \ldots \ldots \ldots \ldots \ldots \ldots$

Duane Arnold.................................. A-37

Joseph M. Farley.1........................... A-39

Joseph M. Farley $2 \ldots \ldots \ldots \ldots \ldots \ldots \ldots \ldots \ldots \ldots \ldots \ldots \ldots \ldots \ldots \ldots \ldots$

J.A. Fitzpatrick $1 \ldots \ldots \ldots \ldots \ldots \ldots \ldots \ldots \ldots \ldots \ldots \ldots \ldots \ldots \ldots \ldots \ldots \ldots \ldots$ A-44

Fort Calhoun.1............................... A-47

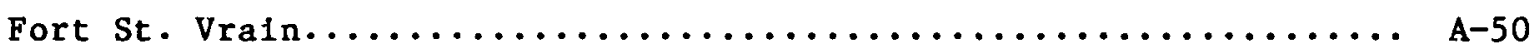

R.E. Ginna.................................. A-51

Haddam Neck................................. A-54

Edwin I. Hatch $1 \ldots \ldots \ldots \ldots \ldots \ldots \ldots \ldots \ldots \ldots \ldots \ldots \ldots \ldots \ldots \ldots$

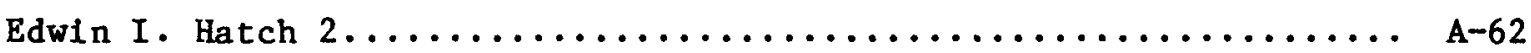

Humboldt Bay $3 . \ldots \ldots \ldots \ldots \ldots \ldots \ldots \ldots \ldots \ldots \ldots \ldots \ldots \ldots \ldots \ldots \ldots \ldots \ldots \ldots$

Indian Polnt $1 \& 2 \ldots \ldots \ldots \ldots \ldots \ldots \ldots \ldots \ldots \ldots \ldots \ldots \ldots \ldots \ldots \ldots$

Indian Point $3 \ldots \ldots \ldots \ldots \ldots \ldots \ldots \ldots \ldots \ldots \ldots \ldots \ldots \ldots \ldots \ldots \ldots \ldots \ldots \ldots \ldots$

Kewaunee $1 . \ldots \ldots \ldots \ldots \ldots \ldots \ldots \ldots \ldots \ldots \ldots \ldots \ldots \ldots \ldots \ldots \ldots \ldots \ldots \ldots \ldots \ldots$

LaCrosse................................... A-79

Maine Yankee................................... A-82

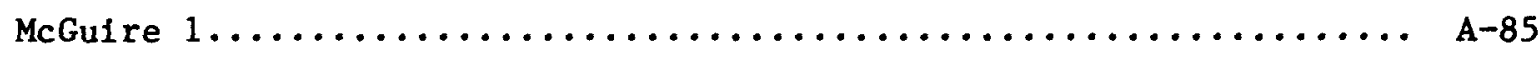

Millstone $1 \ldots \ldots \ldots \ldots \ldots \ldots \ldots \ldots \ldots \ldots \ldots \ldots \ldots \ldots \ldots \ldots \ldots \ldots \ldots \ldots \ldots$

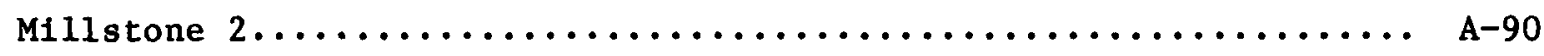

Monticello.................................. A-93

Nine Mile Point $1 \ldots \ldots \ldots \ldots \ldots \ldots \ldots \ldots \ldots \ldots \ldots \ldots \ldots \ldots \ldots \ldots \ldots$

North Anna $1 \& 2 \ldots \ldots \ldots \ldots \ldots \ldots \ldots \ldots \ldots \ldots \ldots \ldots \ldots \ldots \ldots \ldots \ldots \ldots \ldots$ 
Oconee $1,2, \& 3 \ldots \ldots \ldots \ldots \ldots \ldots \ldots \ldots \ldots \ldots \ldots \ldots \ldots \ldots \ldots \ldots \ldots \ldots \ldots \ldots$ A-101

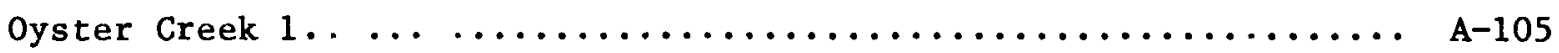

Palisades................................... A-108

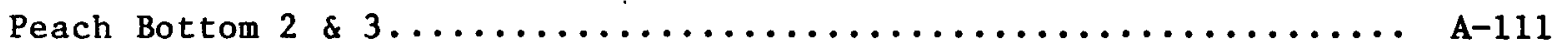

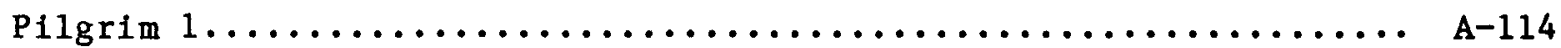

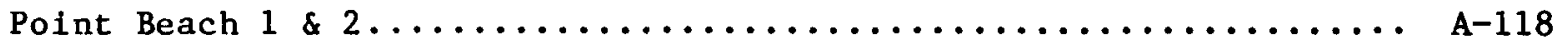

Prairie Island $1 \& 2 \ldots \ldots \ldots \ldots \ldots \ldots \ldots \ldots \ldots \ldots \ldots \ldots \ldots \ldots \ldots \ldots \ldots \ldots \ldots$

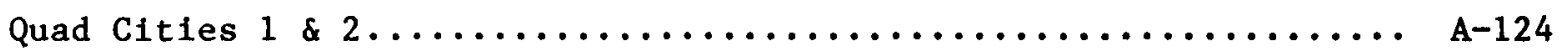

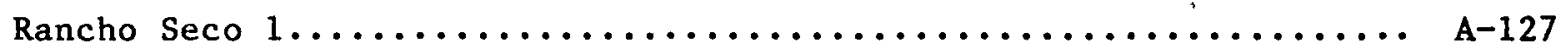

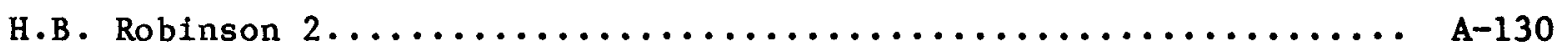

Salem $1 \ldots \ldots \ldots \ldots \ldots \ldots \ldots \ldots \ldots \ldots \ldots \ldots \ldots \ldots \ldots \ldots \ldots \ldots \ldots \ldots \ldots \ldots \ldots \ldots \ldots$

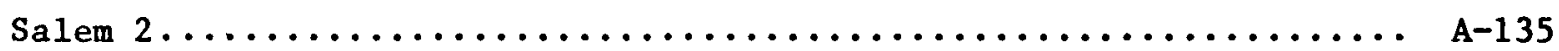

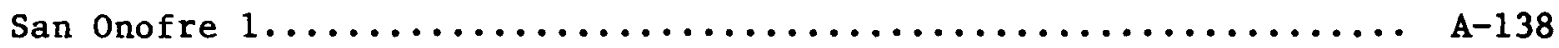

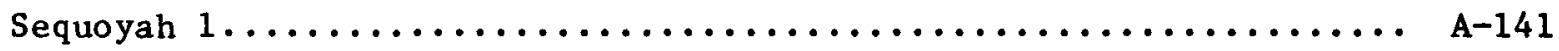

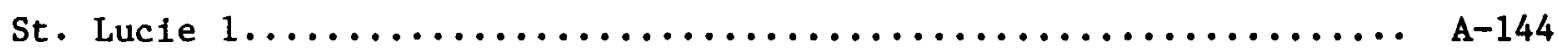

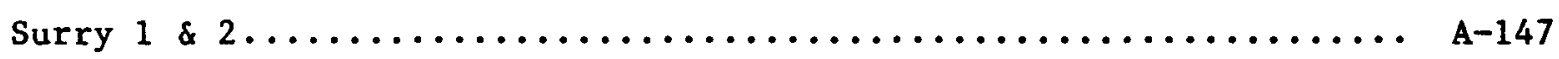

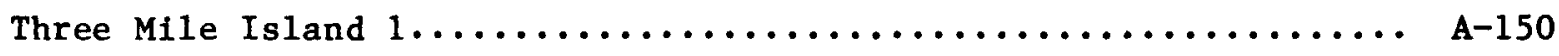

Three Mile Island $2 \ldots \ldots \ldots \ldots \ldots \ldots \ldots \ldots \ldots \ldots \ldots \ldots \ldots \ldots \ldots \ldots \ldots \ldots \ldots$

Three Mile Island $2 /$ EPICOR........................... A-154

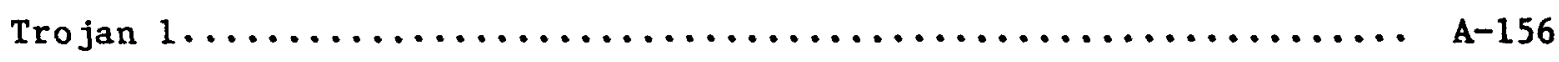

Turkey Point $3 \& 4 \ldots \ldots \ldots \ldots \ldots \ldots \ldots \ldots \ldots \ldots \ldots \ldots \ldots \ldots \ldots \ldots \ldots \ldots$

Vermont Yankee $1 \ldots \ldots \ldots \ldots \ldots \ldots \ldots \ldots \ldots \ldots \ldots \ldots \ldots \ldots \ldots \ldots \ldots \ldots \ldots$

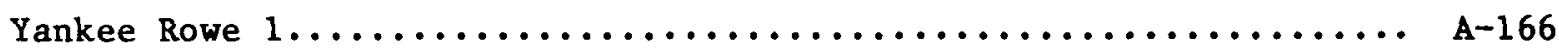

Zion $1 \& 2 \ldots \ldots \ldots \ldots \ldots \ldots \ldots \ldots \ldots \ldots \ldots \ldots \ldots \ldots \ldots \ldots \ldots \ldots \ldots \ldots \ldots$ 


\subsection{Introduction}

\subsection{Purpose}

Th1s report, prepared annually by the staff of the U.S. Nuclear Regulatory Commission, presents measured data on radioactive materials in effluents released from licensed commerclal reactor power plants. These data were reported by licensees for plant operations during 1981. This information supplements earlier annual reports issued by the former Atomfc Energy Commission and Nuclear Regulatory Commission. 1

\subsection{Scope}

Releases of radioactive materials are governed by 10 CFR Part 20 and 50 and by limits established in the Technical Specifications for each facility. The requirement for reporting effluent releases by nuclear power plant operators is described in $10 \mathrm{CFR} 50.36 \mathrm{a}$. Through its office of Inspection and Enforcement, the Nuclear Regulatory Commission maintains a knowledge of radioactive releases from licensed nuclear reactors to ensure that they are within regulatory requirements. This report summarizes data from the 71 licensed nuclear power plants that were declared by the utilities to be in commerclal operation as of December 31, 1981. Data are included for several licensed facilities which are permanently or indefinitely shut down (Dresden 1, Humboldt Bay, Indian Point 1, Three Mile Island 2).

\subsection{Source of Data}

The information included in this report was obtained from data reported by the licensees. Individual licensee reports are available in the NRC Public Document Room, $1717 \mathrm{H}$ Street, NW, Washington, D.C. 20555 and in local Public Document Rooms located near each licensed facility. Licensee reports varied in the format and extent of information provided. This variability will diminish as changes in the Technical Specification for each plant will require adoption of the reporting format of Regulatory Guide 1.21, "Measuring, Evaluating and Reporting Radioactivity in Solid Wastes and Releases of Radioactive Materials in Liquid and Gaseous Effluents from Light Water Cooled Nuclear Power Plants," June 1974.

Data from prior years used in the comparison tables were obtained from the nine previous annual summaries. 1

\footnotetext{
1 "Report on Releases of Radioactivity in Effluents and Solld Wastes from Nuclear Power Plants for 1972," Directorate of Regulatory Operations, August 1973. "Summary of Radloactivity Releases in Effluents from Nuclear Power Plants During 1973," NUREG-75/001, January 1975. "Radloactive Materials Released from Nuclear Power Plants 1974," NUREG-0077, June 1976. "Radioactive Materials Released from Nuclear Power Plants 1975," NUREG-0218, March 1977. "Radioactive Materials Released from Nuclear Power Plants, 1976," NUREG-0367, March 1978. "Radioactive Materials Released from Nuclear Power Plants, 1977," NUREG-0521, January 1979. "Radioactive Materials Released from Nuclear Power Plants, 1978," NUREG/CR-1497, BNL-NUREG-51192, March, 1981. "Radioactive Materials Released from Nuclear Power Plants, 1979, NUREG/CR-2227, BNL-NUREG-51416, November 1981. "Radioactive Materials Released from Nuclear Power Plants, 1980, NUREG/CR-20107, BNL-NUREG-51581, Vol. 1, January 1983.
} 


\subsection{Tabulated Data}

\subsection{Airborne and Liquid Effluents}

Table 1 through 4 list for each reactor, the measured quantities of total noble gases and of I-131 and particulates (with half lives greater than 8 days) released in effluents to the atmosphere during each of the years 1970 through 1981. Tables 5 through 8 11st the total measured quant1ties of tritium and of mixed fission and activation products released in liquid effluents in each of the years.

\subsection{Solid Waste}

The total volumes, activity and the number of shipments of solid waste for each plant during 1981 are summarized in Tables 9 and 10. A comparison for the years 1972 through 1981 is made in Tables 11 and 12 .

\subsection{Energy Generation}

Tables 15 and 16 present a summary of the thermal energy generated by each plant during 1981 and previous years from 1970 . Tables 13 and 14 present a summary of net electrical energy generated by each plant during 19781981. The reader is cautioned against making simplistic comparisons of radioactive releases with the energy generated because of the many factors which affect the amount of radioactive materials released; factors such as the condition of the fuel, primary system integrity, effluent and radloactive waste treatment systems, and the extent to which these systems are used.

\subsection{Individual Plant Summaries}

Individual plant summarles are presented in alphabetical order. The summaries include general plant information, power production, effluent and solid waste data, and a summary of specific radionuclides measured in effluents. When the only type of solid waste reported is type "A", this may be because the plant did not break solid waste into different types but reported all types together. The activity released for each nuclide for the year for both airborne and 1iquid effluents is calculated by sumning releases for each quarter. More detalled summarles in the format of Regulatory Gulde 1.21 such as were used in the 1978 report $^{2}$ can be made avallable since all the data for 1978-1981 are stored in a computerized data base. Individuals interested in obtaining the more detailed summaries should contact the Office of Management and Program Analysis of the Nuclear Regulatory Commission.

\footnotetext{
2"Radioactive Materials Released from Nuclear Power Plants, 1978," NUREG/CR-1497, BNL-NUREG-51192, March, 1981 .
} 


\subsection{Notation}

The following notation is used:

$$
\begin{aligned}
& 1.86 \mathrm{E}+06=1.86 \times 10^{6} \\
& 1.86 \mathrm{E}-03=1.86 \times 10^{-3} \\
& N / R=\text { Not Reported } \\
& N / D=\text { Not Detected } \\
& <\text { may actua1ly mean } \leq
\end{aligned}
$$

\subsection{Summary}

Nearly all of the radioactive material reported as being released in effluents is planned and results from normal operation or is the result of anticipated operational occurrences, $1 . e .$, unplanned releases of radioactive materials from miscellaneous actions such as equipment fallure, operator error, or procedure error that are not of such consequence so as to be considered an accident.

At present, it is difficult to compare effluent releases with those of previous years due to, among other contributors, variability in reporting structure and release requirements. Comparisons with respect to power generation are similarly difficult due to factors which strongly affect the releases such as level of fuel cladding defects, design features of plant radioactive waste treatment systems, operational occurrences and equipment performance.

Though perhaps not identifiable as an important factor at any specific plant from the data in this report, the generic improvement in fuel performance over the last several years has either reduced or has had the potential to reduce the amount of radioactive material released in effluents from most plants. In addition, at Bolling Water Reactors (BWRs), the reduction in the amount of airborne radioactive materials being released at some plants since the early and mid-1970s is due in large part to the installation of augmented offgas (AOG) systems. 


\subsection{List of Tables}

TABLE 1 Alrborne Effluent Comparison by Year, Noble Gases,

Bolling Water Reactors....................... 5

TABLE 2 Alrborne Effluent Comparison by Year, Noble Gases,

Pressurized Water Reactors..................... 7

TABLE 3 Airborne Effluent Comparison by Year, I-131 and

Particulates, Bolling Water Reactors............... 9

TABLE 4 Alrborne Effluent Comparison by Year, I-131 and

Particulates, Pressurized Water Reactors.............. 11

TABLE 5 Liquid Effluent Comparison by Year, Tritium,

Bolling Water Reactors......................... 13

TABLE 6 Liquid Effluent Comparison by Year, Tritium,

Pressurized Water Reactors...................... 15

TABLE 7 Liquid Effluent Comparison by Year, Mixed Fission

and Activation Products, Bolling Water Reactors......... 17

TABLE 8 Liquid Effluent Comparison by Year, Mixed Fission and

Activation Products, Pressurized Water Reactors......... 19

TABLE 9 Solid Waste Summary 1981, Bolling Water Reactors........ 21

TABLE 10 Sol1d Waste Summary 1981, Pressurized Water Reactors..... 22

TABLE 11 Solid Waste Comparison by Year, Boiling Water Reactors.... 23

TABLE 12 Solid Waste Comparison by Year, Pressurized

Water Reactors............................. 25

TABLE 13 Net Electrica1 Energy Generation Comparison

by Year, Bolling Water Reactors.................. 27

TABLE 14 Net Electrical Energy Generation Comparison

by Year, Pressurized Water Reactors..................

TABLE 15 Thermal Energy Generation Comparison by Year,

Bolling Water Reactors....................... 31

TABLE 16 Thermal Energy Generation Comparison by Year,

Pressurized Water Reactors...................... 
TABLE 1

AIRBORNE EFFLUENTS COMPARISON BY YEAR

\section{BOILING WATER REACTORS}

\section{FACILITY}

BIG ROCK POINT

BROWNS FERRY

BRUNSWICK

COOPER

DRESDEN I

DRESDEN 2-3

DUANE ARNOLD

EDWIN I. HATCH 1

EDWIN I . HATCH 2

HUMBOLDT BAY

JAMES A. FITZPATRICK

LACROSSE

MILLSTONE 1

MONTICELLO

NINE MILE POINT

OYSTER CREEK

PEACH BOTTOM

PILGRIM

QUAD-CITIES

VERMONT YANKEE

FORT ST. VRAIN

FISSION AND ACTIVATION GASES (TOTAL CURIES)

\begin{tabular}{|c|c|c|c|c|c|c|c|c|c|}
\hline 1970 & 1971 & 1972 & 1973 & 1974 & 1975 & 1976 & 1977 & 1978 & 1979 \\
\hline \multirow[t]{4}{*}{$2.80 E+05$} & $2.84 E+05$ & $2.58 \mathrm{E}+05$ & $2.30 \mathrm{E}+05$ & 1.88E+05 & 5.06E+04 & 1. $.52 E+04$ & $1.34 E+04$ & $1.89 E+04$ & 6.67E+03 \\
\hline & & & & $6.40 E+04$ & $9.24 E+04$ & $<8.05 E+04$ & $<1.66 \mathrm{E}+05$ & $1.57 \mathrm{E}+05$ & $<2.71 \mathrm{E}+05$ \\
\hline & & & & & $1.90 \mathrm{E}+02$ & $1.90 \mathrm{E}+04$ & $2.46 E+05$ & $9.14 E+04$ & $1.16 \mathrm{E}+05$ \\
\hline & & & & $2.00 \mathrm{E}+03$ & $1.98 \mathrm{E}+04$ & 3.80E+04 & $1.27 E+03$ & 4.09E+03 & 3.04E+04 \\
\hline \multirow[t]{4}{*}{$9.00 \mathrm{E}+05$} & $7.53 E+05$ & $8.77 E+05$ & $8.40 E+05$ & $9.80 E+04$ & 5.20E+05 & $4.52 E+05$ & 5.20E+05 & 8.50E +05 & $1.83 E+02$ \\
\hline & $5.80 E+05$ & $4.29 \mathrm{E}+05$ & $8.80 E+05$ & $6.27 E+05$ & $3.69 E+05$ & 3.23E+04 & $3.13 E+05$ & $4.06 E+04$ & $6.91 \mathrm{E}+04$ \\
\hline & & & & & $1.58 \mathrm{E}+03$ & $5.26 E+03$ & $3.87 \mathrm{E}+03$ & $1.56 \mathrm{E}+03$ & 8.71E+09 \\
\hline & & & & & 2.70E+02 & $2.80 E+03$ & $1.90 E+03$ & $1.62 E+03$ & $1.71 \mathrm{E}+03$ \\
\hline \multirow[t]{2}{*}{ 5. $40 E+05$} & $5.14 E+05$ & 4.30E+05 & 3. $50 E+05$ & 5.72E+05 & $2.97 E+05$ & 9.30E+04 & $4.40 E-05$ & $4.40 \mathrm{E}-0 \mathrm{~S}$ & $<4.4$ \\
\hline & & & & & $4.08 E+03$ & $4.41 E+04$ & $2.33 E+04$ & $5.88 E+03$ & $3.38 E+03$ \\
\hline \multirow[t]{3}{*}{$1.00 E+03$} & $1.00 \mathrm{E}+03$ & 3. 10E+04 & $9.10 E+04$ & $4.90 \mathrm{E}+04$ & $5.71 \mathrm{E}+04$ & $1.24 E+05$ & $4.25 E+04$ & $8.45 E+03$ & $1.04 E+04$ \\
\hline & $2.76 E+05$ & $7.26 E+05$ & $7.90 E+04$ & $9.12 E+05$ & $2.97 E+06$ & $5.07 E+05$ & $6.20 \mathrm{E}+05$ & $5.66 E+05$ & $2.06 E+04$ \\
\hline & $7.60 \mathrm{E}+04$ & $7.51 \mathrm{E}+05$ & $8.70 E+05$ & $1.57 E+06$ & $1.55 \mathrm{E}+05$ & 1. $14 E+04$ & $6.87 \mathrm{E}+03$ & $6.42 E+03$ & $4.03 \mathrm{E}+03$ \\
\hline $1.00 \mathrm{E}+04$ & $2.53 E+05$ & $5.17 E+05$ & $8.72 E+05$ & 5.58E+05 & 1.30E+06 & 1. $76 E+05$ & $3.53 \mathrm{E}+03$ & $3.02 E+03$ & $1.04 E+03$ \\
\hline \multirow[t]{6}{*}{ 1. $10 \mathrm{E}+05$} & $5.16 E+05$ & $8.66 E+05$ & 8. $10 E+05$ & 2.79E+05 & $2.06 E+05$ & $1.67 \mathrm{E}+05$ & $1.77 E+05$ & 9.98E+0B & $1.01 \mathrm{E}+06$ \\
\hline & & & $<1.00 E+03$ & $<1.00 E+00$ & 1. $30 \mathrm{E}+04$ & $2.09 E+05$ & $7.11 E+04$ & $3.85 E+04$ & $1.90 \mathrm{E}+0 \mathrm{~s}$ \\
\hline & & $1.80 E+04$ & $2.30 E+05$ & $5.46 E+05$ & $4.60 E+04$ & $1.83 E+05$ & $4.13 E+05$ & 3.27E+04 & $1.39 E+04$ \\
\hline & & 1.32E+05 & $9.00 E+05$ & $9.50 \mathrm{E}+05$ & 1. $10 E+05$ & 3. $36 E+04$ & $2.56 E+04$ & $3.24 E+04$ & $3.48 E+04$ \\
\hline & & 5.50E+04 & $1.80 E+05$ & $6.40 E+04$ & 4.08E+03 & 3.03E+03 & $3.35 \mathrm{E}+03$ & $4.94 E+03$ & $<8.08 E+03$ \\
\hline & & & & & & & & & $9.30 E+01$ \\
\hline
\end{tabular}


TABLE 1

BOILING WATER REACTORS

\begin{tabular}{lrc} 
FACILITY & \multicolumn{1}{l}{1980} & 1981 \\
BIG ROCK POINT & $2.15 E+04$ & $1.97 E+04$ \\
BROWNS FERRY & $<1.66 E+05$ & $4.52 E+04$ \\
BRUNSWICK & $6.93 E+04$ & $5.22 E+05$ \\
COOPER & $5.03 E+03$ & $2.48 E+03$ \\
DRESDEN 1 & $7.03 E+01$ & N/D \\
DRESDEN 2-3 & $4.30 E+04$ & $3.74 E+04$ \\
DUANE ARNOLD & $2.70 E+03$ & $<4.87 E+02$ \\
EDWIN I. HATCH 1 & $3.82 E+04$ & $2.77 E+04$ \\
EDWIN I. HATCH 2 & $2.95 E+02$ & $2.06 E+02$ \\
HUMBOLDT BAY & $<4.40 E-05$ & $<4.40 E-05$ \\
JAMES A. FITZPATRICK & $7.68 E+04$ & $2.00 E+05$ \\
LACROSSE & $4.71 E+03$ & $5.03 E+03$ \\
MILLSTONE 1 & $1.19 E+04$ & $1.43 E+04$ \\
MONTICELLO & $3.83 E+03$ & $3.74 E+03$ \\
NINE MILE POINT & $5.87 E+02$ & $6.10 E+02$ \\
OYSTER CREEK & $3.12 E+04$ & $5.28 E+04$ \\
PEACH BOTTOM & $1.53 E+04$ & $1.58 E+04$ \\
PILGRIM & $2.62 E+04$ & $<5.30 E+03$ \\
QUAD-CITIES & $2.15 E+04$ & $3.20 E+04$ \\
VERMONT YANKEE & $1.63 E+03$ & $<3.17 E+03$ \\
FORT ST. VRAIN* & $9.13 E+01$ & $4.34 E+01$
\end{tabular}

AIRBORNE EFFLUENTS COMPARISON BY YEAR

FISSION AND ACTIVATION GASES (TOTAL CURIES) 
TABLE 2

AIRBORNE EFFLUENTS COMPARISON BY YEAR

PRESSURIZED WATER REACTORS

FISSION AND ACTIVATION GASES (TOTAL CURIES)

\begin{tabular}{|c|c|c|c|c|c|c|c|c|c|c|}
\hline FACILITY & 1970 & 1971 & 1972 & 1973 & 1974 & 1975 & 1976 & 1977 & 1978 & 1979 \\
\hline ARKANSAS ONE 1 & & & & & 1.96E+02 & 1.03E+03 & $5.69 E+03$ & $1.39 E+04$ & $7.50 E+03$ & $8.51 E+03$ \\
\hline ARKANSAS ONE 2 & & & & & & & & & & 4. 53E+03 \\
\hline BEAVER VALLEY & & & & & & & $1.07 E+00$ & $4.73 E+01$ & $3.90 \mathrm{E}+02$ & $1.75 E+03$ \\
\hline CALVERT CLIFFS & & & & & . & $7.72 E+03$ & $9.40 E+03$ & $2.23 E+04$ & $2.76 E+04$ & $1.02 E+04$ \\
\hline CRYSTAL RIVER & & & & & & & & $3.95 E+03$ & $6.86 E+03$ & $7.26 \mathrm{E}+04$ \\
\hline DAVIS-BESSE & & & & & & & & $1.27 E+03$ & $2.10 E+03$ & $<1.68 E+03$ \\
\hline DONALD C. COOK & & & & & & $2.64 \mathrm{E}+00$ & $9.75 E+02$ & $3.80 E+03$ & $4.85 E+04$ & $1.09 \mathrm{E}+04$ \\
\hline FORT CALHOUN & & & & $6.70 E+01$ & 3.03E+02 & 4.29E+02 & $1.94 E+03$ & $3.81 E+03$ & $1.36 E+03$ & $7.06 E+02$ \\
\hline H. B. ROBINSON & & $1.00 E+00$ & $<1.00 E+00$ & $3.10 E+03$ & $2.31 E+03$ & 1. $17 E+03$ & $6.40 E+02$ & $4.76 E+02$ & $8.84 E+02$ & $1.52 E+03$ \\
\hline HADDAM NECK & 1. $.00 E+00$ & $3.00 E+00$ & $1.00 E+00$ & $9.20 E+01$ & $7.00 E+00$ & $4.80 E+02$ & 4.52E+02 & 3. $12 E+03$ & $2.14 E+03$ & 5.53E+03 \\
\hline INDIAN POINT 1-2 & & & & $1.50 \mathrm{E}+01$ & 5.58E+03 & $8.20 E+03$ & $1.16 E+04$ & $1.60 \mathrm{E}+04$ & $1.41 \mathrm{E}+04$ & $9.03 E+03$ \\
\hline INDIAN POINT 3 & & & & & & & & & $8.09 E+02$ & $2.47 E+02$ \\
\hline $\begin{array}{l}\text { JOSEPH M. FARLEY } 1 \\
\text { JOSEPH }\end{array}$ & & & & & & & & & 3.53E+03 & 3. $18 \mathrm{E}+03$ \\
\hline KEWAUNEE & & & & & $935 F+03$ & & & & & \\
\hline & & & & & & $.452+03$ & $1.40 E+03$ & $2.49 E+03$ & $4.44 E+02$ & $1.52 \mathrm{E}+02$ \\
\hline \multicolumn{7}{|l|}{ MCGUIRE } & E+03 & +02 & to3 & +03 \\
\hline MILLSTONE 2 & & & & & & & 1.57E+03 & $2.28 E+03$ & $7.64 \mathrm{E}+02$ & 3. 59E+02 \\
\hline NORTH ANNA & & & & & & & & & $1.31 \mathrm{E}+04$ & $6.28 E+03$ \\
\hline OCONEE & & & & $9.30 E+03$ & $1.94 E+04$ & $1.51 \mathrm{E}+04$ & $4.39 E+04$ & 3.56E+04 & $4.33 E+04$ & $4.79 \mathrm{E}+04$ \\
\hline PALISADES & & & $1.00 E+00$ & $4.54 E+02$ & $<1.00 E+00$ & $2.61 E+03$ & $2.99 E+01$ & $5.99 \mathrm{E}+01$ & $3.23 E+02$ & $6.84 E+01$ \\
\hline POINT BEACH & & $<1.00 E+00$ & 3.00E +00 & $5.75 E+03$ & $9.74 E+03$ & $4.45 E+04$ & $1.91 E+03$ & $1.13 E+03$ & $5.16 E+02$ & $9.68 E+02$ \\
\hline PRAIRIE ISLAND & & & & $8.72 E+00$ & $3.62 E+02$ & $2.17 E+03$ & 1. $74 E+03$ & $6.73 E+02$ & $1.26 E+03$ & $6.97 E+02$ \\
\hline R. E. GINNA & $1.00 E+01$ & 3. 20E +01 & 1. $20 E+01$ & 5.76E+02 & $7.57 \mathrm{E}+02$ & $1.04 E+04$ & 5. $52 E+03$ & $3.20 E+03$ & $9.72 E+02$ & $7.62 \mathrm{E}+02$ \\
\hline RANCHO SECO & & & & & & 1. $18 E+02$ & 1. $27 E+02$ & $2.00 E+03$ & $7.10 E+03$ & $8.81 \mathrm{E}+03$ \\
\hline SALEM 1 & & & & & & & $<1.00 E-02$ & $1.96 \mathrm{E}+01$ & $1.02 \mathrm{E}+01$ & $2.49 \mathrm{E}+02$ \\
\hline SALEM 2 & & & • & & & & & & & \\
\hline SAN ONOFRE & $<1.00 \mathrm{E}+00$ & $8.00 E+00$ & $1.90 \mathrm{E}+01$ & $1.10 \mathrm{E}+04$ & $1.78 E+03$ & $1.11 \mathrm{E}+03$ & 4. $16 E+02$ & $1.54 \mathrm{E}+02$ & $1.81 E+03$ & $6.37 E+02$ \\
\hline \multicolumn{11}{|l|}{ SEQUOYAH } \\
\hline ST. LUCIE & & & & & & & 1. $72 E+03$ & $2.54 E+04$ & +04 & $1.54 \mathrm{E}+04$ \\
\hline SURRY & & & $<1.00 E+00$ & $8.66 \mathrm{E}+02$ & $6.86 E+03$ & $8.04 E+03$ & $1.91 \mathrm{E}+04$ & $1.90 E+04$ & $4.36 E+03$ & $1.78 E+03$ \\
\hline THREE MILE ISLAND I & & & & & $9.16 E+02$ & $3.63 \mathrm{E}+03$ & $2.76 E+03$ & $1.66 E+04$ & $1.57 E+04$ & $2.24 E+03$ \\
\hline THREE MILE ISLAND 2 & & & & & & & & & B. $73 E+00$ & $9.97 E+06$ \\
\hline \multicolumn{11}{|l|}{ TMI 2/EPICOR } \\
\hline TROJAN & & & & & & & $6.66 \mathrm{E}+02$ & -03 & & $9.27 E+02$ \\
\hline TURKEY POINT & & & & 5.30E+02 & $4.66 E+03$ & 1. $34 \mathrm{E}+04$ & $1.56 E+04$ & $2.33 E+04$ & $2.35 E+04$ & $1.06 \mathrm{E}+04$ \\
\hline YANKEE ROWE & $<1.00 E+00$ & $<1.00 \mathrm{E}+00$ & $<1.00 E+00$ & 3.50E+01 & $4.00 E+01$ & $2.24 E+01$ & $2.57 E+01$ & 1.25E+02 & $6.56 E+02$ & $1.82 E+02$ \\
\hline ZION & & & & $4.00 E+00$ & $2.99 E+03$ & $4.88 E+04$ & $1.14 E+05$ & $3.22 E+04$ & $6.77 \mathrm{E}+04$ & $3.41 \mathrm{E}+04$ \\
\hline
\end{tabular}


TABLE 2

PRESSURIZED WATER REACTORS

198

ARKANSAS ONE 1

ARKANSAS ONE 2

BEAVER VALLEY

CALVERT CLIFFS

CRYSTAL RIVER

DAVIS-BESSE

DONAL.D C. COOK

FORT GALHOUN

H. B. ROBINSON

HADDAM NECK

INDIAN POINT 1-2

INDIAN POINT 8

JOSEPH M. FARLEY 1

JOSEPH M. FARLEY 2

KEWAUNEE

MAINE YANKEE

MCGUIRE

MILLSTONE 2

NORTH ANNA

OCONEE

PALISADES

POINT BEACH

PRAIRIE ISLAND

R. E. GINNA

RANCHO SECO

SALEM 1

SALEM 2

SAN ONOFRE

SEQUOYAH

ST. LUCIE

SURRY

THREE MILE ISLAND

THREE MILE ISLAND 2

TMI 2/EPICOR

TROJAN

TURKEY POIN

YANKEE ROWE

ZION
FISSION AND ACTIVATION GASES (TOTAL CURIES)

$9.37 E+03 \quad 4.35 E+03$

$8.64 \mathrm{E}+01 \quad 8.06 \mathrm{E}+02$

$2.96 \mathrm{E}+03 \quad 2.18 \mathrm{E}+03$

$3.65 E+04 \quad 3.96 E+04$

$3.35 E+03 \quad 1.01 E+03$

3. $76 \mathrm{E}+035.42 \mathrm{E}+03$

$2.97 E+02 \quad 1.22 E+03$

$5.82 E+02 \quad 5.13 E+02$

$2.68 \mathrm{E}+03 \quad 1.83 \mathrm{E}+03$

9.38E+03 9.13E+03

$111 \mathrm{E}+03 \quad 6.57 \mathrm{E}+03$

$1.92 E+04 \quad 2.21 E+02$

$2.60 \mathrm{E}+00$

1.22E+02 1.18E+02

$3.87 \mathrm{E}+03 \quad 3.28 \mathrm{E}+02$

1. $58 \mathrm{E}-01$

$1.33 \mathrm{E}+03 \quad 2.24 \mathrm{E}+03$

$3.50 \mathrm{E}+035.30 \mathrm{E}+03$

$1.92 \mathrm{E}+04 \quad 1.63 \mathrm{E}+04$

1. 40E +02 3.00E+03

$6.41 \mathrm{E}+02 \quad 6.11 \mathrm{E}+02$

$2.60 \mathrm{E}+02 \quad 4.65 \mathrm{E}+01$

$8.61 \mathrm{E}+02 \quad 5.46 \mathrm{E}+02$

$1.58 E+03 \quad 1.37 E+03$

$7.82 \mathrm{E}+01,1.06 \mathrm{E}+03$

$7.74 E+00 \quad 6.09 E+02$

$1.05 \mathrm{E}+03 \quad 4.17 \mathrm{E}+02$

$3.01 \mathrm{E}+03 \quad 9.03 \mathrm{E}+03$

$8.97 E+032.30 E+04$

$6.17 E+03 \quad 1.41 E+04$

4.64E-03 5.81E-02

4.72E+04 2.88E+02

$2.16 E+00 \quad 1.84 E+02$

$3.91 \mathrm{E}+02 \quad 1.16 \mathrm{E}+03$

4.24E+03 4.33E+03

$7.07 \mathrm{E}+01 \quad 1.72 \mathrm{E}+02$

5. 78E+03 6.91E+03

\section{AIRBORNE EFFLUENTS COMPARISON BY YEAR}


TABLE 3

AIRBORNE EFFLUENTS COMPARISON BY YEAR (CURIES)

\section{I-131 AND PARTICULATES}

BOILING WATER REACTORS

\begin{tabular}{|c|c|c|c|c|c|c|c|c|c|c|}
\hline FACILITY & 1970 & 1971 & 1972 & 1973 & 1974 & 1975 & 1976 & 1977 & 1978 & 1979 \\
\hline BIG ROCK POINT & $1.30 \mathrm{E}-01$ & $6.10 \mathrm{E}-01$ & $1.50 \mathrm{E}-01$ & $4.60 E+00$ & $1.60 \mathrm{E}-01$ & $1.20 \mathrm{E}-01$ & $5.00 \mathrm{E}-02$ & 1.00E-02 & $8.91 \mathrm{E}-03$ & $1.90 \mathrm{E}-03$ \\
\hline BROWNS FERRY & & & & & $1.20 \mathrm{E}-01$ & $2.70 \mathrm{E}-01$ & $<7.00 \mathrm{E}-02$ & $1.04 \mathrm{E}-01$ & $2.27 \mathrm{E}-01$ & 5.03E-02 \\
\hline BRUNSWI CK & & & & & & $<1.00 \mathrm{E}-02$ & $4.60 \mathrm{E}-01$ & 9.32E-01 & 4.07E-01 & $9.52 \mathrm{E}-01$ \\
\hline COOPER & & & & & $2.40 E-01$ & 5.00E-02 & $<4.00 \mathrm{E}-02$ & $<1.91 \mathrm{E}-02$ & $5.41 \mathrm{E}-03$ & $<1.79 \mathrm{E}-01$ \\
\hline DRESDEN 1 & $3.30 E+00$ & $6.70 E-01$ & $2.75 E+00$ & $4.00 E-02$ & $6.80 \mathrm{E}-01$ & $9.60 \mathrm{E}-01$ & $8.40 \mathrm{E}-01$ & $4.93 \mathrm{E}+00$ & $2.28 \mathrm{E}+00$ & $2.38 E-02$ \\
\hline DRESDEN 2-3 & $1.60 \mathrm{E}+00$ & $8.68 E+00$ & $5.89 \mathrm{E}+00$ & $6.70 E+00$ & $6.50 \mathrm{E}+00$ & $4.31 E+00$ & $5.49 E+00$ & $6.86 E+00$ & 3. $13 E+00$ & $6.97 \mathrm{E}+00$ \\
\hline DUANE ARNOLD & & & & & & $1.10 \mathrm{E}-03$ & $8.18 E-02$ & 2. 29E-02 & 3. $65 \mathrm{E}-02$ & 3. 35E-02 \\
\hline EDWIN I. HATCH 1 & & & & & & $<1.00 E-02$ & $<1.00 \mathrm{E}-02$ & 5.67E-03 & $4.13 \mathrm{E}-03$ & $2.59 \mathrm{E}-02$ \\
\hline EDWIN I HATCH 2 & & & & & & & & & & \\
\hline HUMBOLDT BAY & $3.50 \mathrm{E}-01$ & $3.00 \mathrm{E}-01$ & $4.80 E-01$ & $2.90 \mathrm{E}-01$ & $8.40 \mathrm{E}-01$ & $1.06 \mathrm{E}+00$ & $8.36 \mathrm{E}-02$ & 4.04E-03 & $7.26 \mathrm{E}-04$ & $1.07 \mathrm{E}-04$ \\
\hline JAMES A. FITZPATR ICK & & & & & & $<4.00 E-02$ & $6.80 \mathrm{E}-01$ & $1.73 E-01$ & $2.79 E-01$ & 1. $42 E-02$ \\
\hline LACROSSE & $<6.00 \mathrm{E}-02$ & $<1.00 \mathrm{E}-02$ & $7.10 \mathrm{E}-01$ & $2.00 \mathrm{E}-01$ & $4.00 \mathrm{E}-02$ & $1.00 \mathrm{E}-01$ & $<7.06 \mathrm{E}-02$ & 1. 67E-01 & $2.79 \mathrm{E}-02$ & 2.53E-02 \\
\hline MILLSTONE 1 & & $4.00 E+00$ & $1.32 \mathrm{E}+00$ & $2.00 \mathrm{E}-01$ & $3.26 \mathrm{E}+00$ & 9.98E+00 & $2.33 E+00$ & $4.86 E+00$ & $4.55 E+00$ & $5.90 \mathrm{E}-01$ \\
\hline MONT ICELLO & & 3.60E-02 & $5.78 \mathrm{E}-01$ & $1.20 \mathrm{E}+00$ & $5.70 \mathrm{E}+00$ & $3.71 E+00$ & $1.71 \mathrm{E}-01$ & $8.51 \mathrm{E}-02$ & $5.49 \mathrm{E}-02$ & 3. 39E-02 \\
\hline NINE MILE POINT & $<1.00 \mathrm{E}-02$ & $6.00 \mathrm{E}-02$ & $9.70 \mathrm{E}-01$ & $1.98 E+00$ & $8.90 \mathrm{E}-01$ & $2.78 E+00$ & $2.20 E+00$ & 1.99E-01 & $1.35 E-01$ & $4.71 E-02$ \\
\hline OYSTER CREEK & $3.20 \mathrm{E}-01$ & $2.14 E+00$ & $6.48 E+00$ & $7.02 E+00$ & $3.51 \mathrm{E}+00$ & $5.64 \mathrm{E}+00$ & $6.39 \mathrm{E}+00$ & $9.05 E+00$ & $1.81 \mathrm{E}+01$ & $9.32 E+00$ \\
\hline РЕACH ВOTTOM & & & & $<1.00 \mathrm{E}-02$ & $1.00 \mathrm{E}-02$ & 4.00E-02 & $9.75 E-01$ & $2.73 \mathrm{E}-01$ & $9.62 \mathrm{E}-02$ & $2.58 E-01$ \\
\hline PILGRIM & & & 3.00E-02 & $4.70 \mathrm{E}-01$ & 1. $45 E+00$ & $2.58 E+00$ & $6.74 E-01$ & $6.90 \mathrm{E}-01$ & $1.81 \mathrm{E}-01$ & $1.45 \mathrm{E}-01$ \\
\hline QUAD-CITIES & & & $7.50 \mathrm{E}-01$ & $5.50 E+00$ & $8.88 E+00$ & $1.31 \mathrm{E}+00$ & $1.33 \mathrm{E}+00$ & 1. $69 \mathrm{E}+00$ & $2.15 \mathrm{E}+00$ & 1. $57 \mathrm{E}+00$ \\
\hline VERMONT YANKEE & & & $1.70 \mathrm{E}-01$ & $7.00 \mathrm{E}-02$ & $3.60 \mathrm{E}-01$ & $1.00 \mathrm{E}-02$ & $<1.00 \mathrm{E}-02$ & $1.44 \mathrm{E}-02$ & $2.18 \mathrm{E}-01$ & $4.43 E-01$ \\
\hline FORT ST. VRAIN & & & & & & & & & & $6.89 E-07$ \\
\hline
\end{tabular}


TABLE 3

AIRBORNE EFFLUENTS COMPARISON BY YEAR (CURIES)

I-131 AND PARTICULATES

BOILING WATER REACTORS

(HALF-LIFE EQUAL TO OR GREATER THAN 8 DAYS)

\begin{tabular}{lrr} 
FACILITY & \multicolumn{1}{l}{1980} & \multicolumn{1}{l}{1981} \\
BIG ROCK POINT & $2.94 \mathrm{E}-02$ & $6.10 \mathrm{E}-03$ \\
BROWNS FERRY & $1.05 \mathrm{E}-01$ & $4.00 \mathrm{E}-02$ \\
BRUNSWICK & $2.12 \mathrm{E}+00$ & $8.89 \mathrm{E}-01$ \\
COOPER & $1.52 \mathrm{E}-01$ & $<1.09 \mathrm{E}-02$ \\
DRESDEN 1 & $1.46 \mathrm{E}-02$ & $9.94 \mathrm{E}-03$ \\
DRESDEN 2-3 & $1.10 \mathrm{E}+01$ & $9.87 \mathrm{E}+00$ \\
DUANE ARNOLD & $8.50 \mathrm{E}-02$ & $3.25 \mathrm{E}-02$ \\
EDWIN I. HATCH 1 & $4.29 \mathrm{E}-01$ & $2.12 \mathrm{E}-01$ \\
EDWIN I. HATCH 2 & $1.33 \mathrm{E}-02$ & $9.42 \mathrm{E}-03$ \\
HUMBOLDT BAY & $5.11 \mathrm{E}-04$ & $<3.82 \mathrm{E}-04$ \\
JAMES A. FITZPATRICK & $1.25 \mathrm{E}-01$ & $2.80 \mathrm{E}-01$ \\
LACROSSE & $1.32 \mathrm{E}-02$ & $1.69 \mathrm{E}-02$ \\
MILLSTONE 1 & $3.32 \mathrm{E}-01$ & $1.48 \mathrm{E}-01$ \\
MONTICELLO & $2.83 \mathrm{E}-02$ & $3.45 \mathrm{E}-02$ \\
NINE MILE POINT & $2.55 \mathrm{E}-02$ & $1.49 \mathrm{E}-02$ \\
OYSTER CREEK & $1.25 \mathrm{E}+00$ & $2.24 \mathrm{E}+00$ \\
PEACH BOTTOM & $2.94 \mathrm{E}-02$ & $<4.19 \mathrm{E}-02$ \\
PILGRIM & $1.04 \mathrm{E}-01$ & $<6.87 \mathrm{E}-02$ \\
QUAD-CITIES & $5.90 \mathrm{E}-01$ & $1.27 \mathrm{E}+00$ \\
VERMONT YANKEE & $1.70 \mathrm{E}-02$ & $4.53 \mathrm{E}-03$ \\
FORT ST. VRAIN* & $1.25 \mathrm{E}-06$ & $1.40 \mathrm{E}-06$
\end{tabular}


TABLE 4

AIRBORNE EFFLUENTS COMPARISON BY YEAR (CURIES)

I-131 AND PARTICULATES

PRESSURIZED WATER REACTORS

(HALF-LIFE EQUAL TO OR GREATER THAN 8 DAYS)

\begin{tabular}{|c|c|c|c|c|c|c|c|c|c|c|}
\hline FACILITY & 1970 & 1971 & 1972 & 1973 & 1974 & 1975 & 1976 & 1977 & 1978 & 1979 \\
\hline ARKANSAS ONE 1 & & & & & $5.00 \mathrm{E}-02$ & $7.40 \mathrm{E}-01$ & 5. $73 E-02$ & $9.04 \mathrm{E}-03$ & 3. $19 \mathrm{E}-03$ & $4.47 E-03$ \\
\hline ARKANSAS ONE 2 & & & & & & & & & & $4.65 E-03$ \\
\hline BEAVER VALLEY & & & & & & & $<1.00 \mathrm{E}-02$ & $1.52 E-04$ & $7.21 \mathrm{E}-02$ & $4.07 E-04$ \\
\hline CALVERT CLIFFS & & & & & & $7.00 \mathrm{E}-02$ & $1.38 \mathrm{E}-01$ & $3.07 E-01$ & $1.35 \mathrm{E}-01$ & $2.05 E+00$ \\
\hline CRYSTAL RIVER & & & & & & & & $2.53 \mathrm{E}-03$ & $1.05 E-03$ & $1.88 \mathrm{E}-02$ \\
\hline DAVIS-BESSE & & & & & & & & $2.57 \mathrm{E}-04$ & $4.30 E-04$ & $5.69 \mathrm{E}-08$ \\
\hline DONALD C. COOK & & & & & & $<1.00 \mathrm{E}-02$ & $<1.00 \mathrm{E}-02$ & 7. 45E-02 & 1. 10E-0I & $7.36 \mathrm{E}-02$ \\
\hline FORT CALHOUN & & & & $<1.00 \mathrm{E}-02$ & $<1.00 \mathrm{E}-02$ & $<1.00 \mathrm{E}-02$ & $<2.04 \mathrm{E}-02$ & 1. $34 \mathrm{E}-02$ & $8.30 \mathrm{E}-03$ & 1. 58E-03 \\
\hline H. B. ROBINSON & & & 3.00E-02 & $3.00 \mathrm{E}-01$ & 5.00E-02 & $2.00 \mathrm{E}-02$ & $9.96 \mathrm{E}-02$ & 3. $88 \mathrm{E}-03$ & $9.26 E-04$ & 4. 10E-04 \\
\hline HADDAM NECK & $<1.00 \mathrm{E}-02$ & & $2.00 \mathrm{E}-02$ & $5.00 \mathrm{E}-02$ & $<1.00 \mathrm{E}-02$ & $<1.00 E-02$ & $<1.00 \mathrm{E}-02$ & $1.74 \mathrm{E}-03$ & $5.21 \mathrm{E}-03$ & $4.77 E-02$ \\
\hline INDIAN POINT 1-2 & & & & $<1.00 \mathrm{E}-02$ & $4.30 \mathrm{E}-01$ & $1.62 \mathrm{E}+00$ & 2.42E-01 & $5.59 \mathrm{E}-02$ & $2.05 \mathrm{E}-01$ & $4.50 \mathrm{E}-01$ \\
\hline INDIAN POINT 3 & & & & & & & & & 1. $29 \mathrm{E}-02$ & 3.89E-03 \\
\hline JOSEPH M. FARLEY 1 & & & & & & & & & 4. $11 \mathrm{E}-02$ & $2.20 \mathrm{E}-02$ \\
\hline JOSEPH M. FARLEY 2 & & & & & & & & & & \\
\hline KEWAUNEE & & & & & $2.00 \mathrm{E}-02$ & $6.60 \mathrm{E}-01$ & $<1.00 \mathrm{E}-02$ & $2.40 \mathrm{E}-02$ & $5.48 E-03$ & $6.18 \mathrm{E}-04$ \\
\hline MAINE YANKEE & & & $<1.00 \mathrm{E}-02$ & $9.40 \mathrm{E}-01$ & 5.00E-02 & $<1.00 \mathrm{E}-02$ & $<1.00 \mathrm{E}-02$ & $5.05 \mathrm{E}-03$ & 2.07E-03 & 5. 52E-02 \\
\hline \multicolumn{11}{|l|}{ MCGUIRE } \\
\hline MILLSTONE 2 & & & & & & 1.00E-02 & $1.25 \mathrm{E}-02$ & $4.47 E-03$ & $2.97 E-03$ & $9.79 E-03$ \\
\hline NORTH ANNA & & & & & & & & & 3. 19E-02 & 5.71E-02 \\
\hline OCONEE & & & & $1.00 \mathrm{E}-02$ & $3.00 \mathrm{E}-02$ & $1.00 E-02$ & $2.72 E-01$ & $5.35 \mathrm{E}-01$ & $2.22 \mathrm{E}-01$ & $2.28 \mathrm{E}-01$ \\
\hline PALI SADES & & & $<1.00 \mathrm{E}-02$ & $3.10 \mathrm{E}-01$ & $1.00 \mathrm{E}-02$ & 3. $80 \mathrm{E}-01$ & $4.16 \mathrm{E}-02$ & $1.63 \mathrm{E}-02$ & $2.07 \mathrm{E}-02$ & $2.46 \mathrm{E}-02$ \\
\hline POINT BEACH & & $<1.00 \mathrm{E}-02$ & $3.00 \mathrm{E}-02$ & $5.50 \mathrm{E}-01$ & $1.60 \mathrm{E}-01$ & $7.00 E-02$ & $1.85 E-02$ & $5.02 E-03$ & $2.88 E-02$ & 1. $35 \mathrm{E}-02$ \\
\hline PRAIRIE ISLAND & & & & $<1.00 \mathrm{E}-02$ & $<1.00 \mathrm{E}-02$ & 2. 12E-02 & 1. 14E-02 & 7. $56 \mathrm{E}-03$ & 8.96E-04 & 3.86E-03 \\
\hline R. E. GINNA & 5.00E-02 & $1.70 \mathrm{E}-01$ & 4.00E-02 & $<1.00 \mathrm{E}-02$ & $<1.00 \mathrm{E}-02$ & $2.00 \mathrm{E}-02$ & 3. 17E-02 & $2.55 \mathrm{E}-02$ & $1.04 \mathrm{E}-02$ & $1.88 \mathrm{E}-02$ \\
\hline RANCHO SECO & & & & & & $<1.00 \mathrm{E}-02$ & $<1.00 E-02$ & $5.02 \mathrm{E}-03$ & 3.21E-02 & $5.75 E-03$ \\
\hline SALEM 1 & & & & & & & N/D & $2.34 \mathrm{E}-07$ & $4.01 E-02$ & $7.68 \mathrm{E}-03$ \\
\hline \multicolumn{11}{|l|}{ SALEM 2} \\
\hline SAN ONOFRE & $<1.00 \mathrm{E}-02$ & $<1.00 \mathrm{E}-02$ & $<1.00 \mathrm{E}-02$ & $1.61 \mathrm{E}+00$ & $<1.00 \mathrm{E}-02$ & 4.00E-02 & $<1.00 E-02$ & 1. $86 \mathrm{E}-04$ & $2.71 E-03$ & 1. $43 E-04$ \\
\hline \multicolumn{11}{|l|}{ SEQUOYAH } \\
\hline ST. LUCIE & & & & & & & $<1.00 \mathrm{E}-02$ & $1.48 E-01$ & 5. 17E-01 & $2.02 E-01$ \\
\hline SURRY & & & $<1.00 \mathrm{E}-02$ & $4.00 \mathrm{E}-02$ & $1.40 \mathrm{E}-01$ & $5.00 \mathrm{E}-02$ & $3.46 \mathrm{E}-01$ & $1.20 \mathrm{E}-01$ & $6.49 E-02$ & $7.61 \mathrm{E}-03$ \\
\hline THREE MILE ISLAND I & & & & & $<1.00 \mathrm{E}-02$ & $<1.00 \mathrm{E}-02$ & 1.07E-02 & 3.39E-02 & $1.35 E-01$ & 1. 24E-02 \\
\hline THREE MILE ISLAND 2 & & & & & & & & & $2.30 E-03$ & 1. $42 E+01$ \\
\hline \multicolumn{11}{|l|}{ TMI 2/EPICOR } \\
\hline TROJAN & & & & & & & 1.64E-02 & 5. $05 \mathrm{E}-02$ & $1.03 \mathrm{E}-02$ & 3.39E-02 \\
\hline TURKEY POINT & & & & $6.00 \mathrm{E}-02$ & $3.63 \mathrm{E}+00$ & 4. 30E-01 & $4.22 \mathrm{E}-01$ & $1.04 \mathrm{E}+00$ & $4.59 \mathrm{E}-01$ & $7.91 \mathrm{E}-02$ \\
\hline YANKEE ROWE & $<1.00 \mathrm{E}-02$ & $<1.00 \mathrm{E}-02$ & $<1.00 \mathrm{E}-02$ & $1.90 \mathrm{E}-01$ & $5.30 E-01$ & $1.00 \mathrm{E}-02$ & $<1.00 \mathrm{E}-02$ & $8.70 E-05$ & $2.25 E-04$ & $2.49 E-04$ \\
\hline ZION & & & & $<1.00 E-02$ & $1.00 E-02$ & $1.40 \mathrm{E}-01$ & $9.00 E-02$ & 5. $98 E-02$ & $8.91 E-02$ & 6. $74 E-02$ \\
\hline
\end{tabular}




\section{TABLE 4}

AIRBORNE EFFLUENTS COMPARISON BY YEAR (CURIES)

\section{I-131 AND PARTICULATES}

PRESSURIZED WATER REACTORS

(HALF-LIFE EQUAL TO OR GREATER THAN 8 DAYS)

\begin{tabular}{|c|c|c|}
\hline FACILITY & 1980 & 1981 \\
\hline ARKANSAS ONE 1 & $1.66 E-01$ & 5.58E-03 \\
\hline ARKANSAS ONE 2 & $6.90 \mathrm{E}-03$ & $1.41 \mathrm{E}-02$ \\
\hline BEAVER VALLEY & $1.91 E-03$ & $6.85 E-03$ \\
\hline ALVERT CLIFFS & 7.44E-02 & $4.69 \mathrm{E}-02$ \\
\hline RYSTAL RIVER & 6.77E-03 & 1. 78E-02 \\
\hline AVIS-BESSE & $2.01 E-03$ & 5.79E-02 \\
\hline ONALD C. COOK & $6.88 \mathrm{E}-02$ & 3.55E-01 \\
\hline FORT CALHOUN & $2.42 E-03$ & 3. 59E-0s \\
\hline . B. ROBINSON & 1. $13 \mathrm{E}-03$ & 32E-04 \\
\hline ADDAM NECK & 8.01E-03 & $<1.28 \mathrm{E}-02$ \\
\hline INDIAN POINT 1-2 & $6.42 E-02$ & 4.42E-02 \\
\hline INDIAN POINT 3 & 2.53E-02 & 3. $63 \mathrm{E}-03$ \\
\hline JOSEPH M. FARLEY 1 & $2.37 E-03$ & $6.24 \mathrm{E}-01$ \\
\hline JOSEPH M. FARLEY 2 & & 3.22E-08 \\
\hline KEWAUNEE & $2.61 E-04$ & $1.21 \mathrm{E}-04$ \\
\hline MAINE YANKEE & $1.88 E-03$ & $6.13 \mathrm{E}-04$ \\
\hline MCGUIRE & & $1.21 \mathrm{E}-11$ \\
\hline MILLSTONE 2 & $1.94 E-02$ & $1.06 \mathrm{E}-01$ \\
\hline NORTH ANNA & $1.26 E-02$ & $4.81 \mathrm{E}-01$ \\
\hline OCONEE & $1.33 E-01$ & $3.24 E-01$ \\
\hline PALI SADES & $2.76 E-02$ & 4.15E-02 \\
\hline POINT BEACH & 1.28E-03 & $2.03 \mathrm{E}-01$ \\
\hline PRAIRIE ISLAND & 1. 83E-03 & $4.49 E-04$ \\
\hline R. E. GINNA & $9.00 E-03$ & 5.88E-0 \\
\hline RANCHO SECO & $9.96 \mathrm{E}-03$ & $4.65 \mathrm{E}-03$ \\
\hline SALEM 1 & $2.17 E-01$ & $4.84 E-01$ \\
\hline SALEM 2 & 5.4 & E-03 \\
\hline SAN ONOFRE & & 8E-02 \\
\hline SEQUOYAH & 2. & $1.30 \mathrm{E}-02$ \\
\hline ST. LUCIE & 6.20 & $7.69 E-02$ \\
\hline SURRY & 1.85 & $6.53 \mathrm{E}-02$ \\
\hline THREE MILE ISLAND I & 2.93 & 5.05E-04 \\
\hline THREE MILE ISLAND 2 & E-04 & 3.69E-05 \\
\hline TMI 2/EPICOR & $6.83 E-06$ & $2.63 E-06$ \\
\hline ROJAN & $2.51 \mathrm{E}-02$ & $7.67 \mathrm{E}-02$ \\
\hline URKEY POINT & $7.05 E-02$ & $2.94 \mathrm{E}-02$ \\
\hline ANKEE ROWE & $9.56 E-05$ & $2.13 \mathrm{E}-04$ \\
\hline ION & 3.00E-03 & $1.25 E-0$ \\
\hline
\end{tabular}


TABLE 5

LIQUID EFFLUENTS COMPARISON BY YEAR (CURIES)

TRITIUM

BOILING WATER REACTORS

\begin{tabular}{|c|c|c|c|c|c|c|c|c|c|c|}
\hline FACILITY & 1970 & 1971 & 1972 & 1973 & 1974 & 1975 & 1976 & 1977 & 1978 & 1979 \\
\hline BIG ROCK POINT & $5.40 E+01$ & $1.03 E+01$ & 1.04E+01 & $1.97 E+01$ & $5.10 E+00$ & $5.73 E+00$ & $2.41 E+00$ & $8.83 E+00$ & 4. $05 E+00$ & $5.45 E+00$ \\
\hline BROWNS FERRY & & & & & $2.80 E+00$ & $1.04 E+01$ & $<4.02 E+00$ & $2.40 E+01$ & 3.08E+01 & $1.32 E+01$ \\
\hline BRUNSWICK & & & & & & $3.20 \mathrm{E}+00$ & $5.90 \mathrm{E}+00$ & $8.93 E+00$ & $1.41 E+01$ & $3.09 E+01$ \\
\hline COOPER & & & & & $1.70 \mathrm{E}+00$ & $8.25 E+00$ & $8.43 E+00$ & 9.04E+00 & $7.51 \mathrm{E}+00$ & $6.63 E+00$ \\
\hline DRESDEN 1 & 5.00E+00 & 8. 70E+00 & $4.33 E+01$ & $1.85 \mathrm{E}+01$ & $1.88 \mathrm{E}+01$ & $2.70 E-01$ & $2.00 \mathrm{E}-02$ & $8.90 \mathrm{E}-02$ & $1.31 E+01$ & 1. $50 \mathrm{E}+00$ \\
\hline DRESDEN 2-3 & 3. $10 \mathrm{E}+01$ & $3.85 E+01$ & $2.59 E+01$ & $2.58 E+01$ & $2.26 \mathrm{E}+01$ & $5.40 E+01$ & $1.97 E+01$ & $5.00 E+00$ & $1.92 E+01$ & $1.93 \mathrm{E}+01$ \\
\hline DUANE ARNOLD & & & & & & $3.30 \mathrm{E}-01$ & 3. $40 \mathrm{E}-01$ & $2.13 \mathrm{E}-01$ & 1. $19 \mathrm{E}+02$ & $2.90 \mathrm{E}-01$ \\
\hline EDWIN I . HATCH 1 & & & & & & $6.12 E+00$ & $8.98 E+00$ & $1.20 \mathrm{E}+01$ & $9.00 \mathrm{E}+00$ & 1. $23 \mathrm{E}+01$ \\
\hline EDWIN I. HATCH 2 & & & & & & & & & & \\
\hline HUMBOLDT BAY & $7.00 \mathrm{E}+00$ & $7.50 \mathrm{E}+00$ & $1.30 \mathrm{E}+01$ & 5. $13 E+01$ & 3. $17 E+01$ & $2.01 E+01$ & $1.30 \mathrm{E}+01$ & 5.26E-01 & 3.63E-02 & 3.91E-02 \\
\hline JAMES A. FITZPATR ICK & & & & & & 5.03E+00 & 4. $20 \mathrm{E}+00$ & 3. $35 \mathrm{E}+00$ & 1. $90 \mathrm{E}+00$ & 1. $.52 \mathrm{E}+00$ \\
\hline LACROSSE & $2.00 E+01$ & $9.14 \mathrm{E}+01$ & 1. $20 \mathrm{E}+02$ & $1.03 E+02$ & $1.15 \mathrm{E}+02$ & 1.27E+02 & 4. $10 \mathrm{E}+01$ & $4.86 \mathrm{E}+01$ & $4.72 E+01$ & 3. $54 \mathrm{E}+01$ \\
\hline MILLSTONE 1 & & $1.27 E+01$ & $2.09 \mathrm{E}+01$ & 3. $70 E+00$ & $2.41 \mathrm{E}+01$ & 8.03E+01 & $2.01 \mathrm{E}+01$ & $4.41 \mathrm{E}+00$ & $\mathbf{E}+\mathbf{0 0}$ & $7.92 \mathrm{E}+00$ \\
\hline MONTICELLO & & $5.92 \mathrm{E}-01$ & $<1.00 \mathrm{E}-01$ & $0.00 E+00$ & $0.00 \mathrm{E}+00$ & $0.00 E+00$ & $0.00 \mathrm{E}+00$ & $0.00 \mathrm{E}+00$ & $0.00 E+00$ & $0.00 \mathrm{E}+00$ \\
\hline NINE MILE POINT & $2.00 \mathrm{E}+01$ & $1.24 E+01$ & $2.78 E+01$ & $4.65 E+01$ & $1.87 E+01$ & $2.81 E+01$ & $2.46 E+00$ & $2.49 E+00$ & $\mathbf{N} / \mathbf{R}$ & $6.78 E+00$ \\
\hline OYSTER CREEK & $2.20 \mathrm{E}+01$ & $2.15 \mathrm{E}+01$ & $6.16 E+01$ & 3. $59 \mathrm{E}+01$ & $1.41 \mathrm{E}+01$ & $1.79 E+01$ & $3.86 E+01$ & $1.88 E+01$ & $1.96 \mathrm{E}+01$ & $1.40 E+00$ \\
\hline РЕАCH ВОТТОМ & & & & $<1.00 E-01$ & $1.00 E+01$ & $3.08 E+01$ & $7.37 \mathrm{E}+01$ & $7.09 \mathrm{E}+01$ & 3. $24 E+01$ & $4.28 E+01$ \\
\hline PILGRIM & & & $4.20 E+00$ & $4.00 \mathrm{E}-01$ & $1.05 \mathrm{E}+01$ & $1.82 E+01$ & $4.67 E+01$ & $3.27 \mathrm{E}+01$ & $2.98 \mathrm{E}+00$ & $1.34 \mathrm{E}+01$ \\
\hline QUAD-CITIES & & & $4.70 E+00$ & $2.45 E+01$ & $3.40 E+01$ & 5.37E+01 & $4.98 \mathrm{E}+01$ & $2.64 E+01$ & $172 E+01$ & $1.76 E+01$ \\
\hline VERMONT YANKEE & & & & $1.00 E-01$ & $0.00 \mathrm{E}+00$ & $0.00 \mathrm{E}+00$ & $1.60 \mathrm{E}+00$ & 8. 44E-01 & $\mathbf{N} / \mathbf{R}$ & 4. $04 \mathrm{E}+00$ \\
\hline FORT ST. VRAIN* & & & & & & & & & & 1. $23 E+02$ \\
\hline
\end{tabular}


TABLE 5

LIQUID EFFLUENTS COMPARISON BY YEAR (CURIES)

\section{TRITIUM}

BOILING WATER REACTORS

$\begin{array}{lcc}\text { FACILITY } & 1980 & 1981 \\ \text { BIG ROCK POINT } & 6.18 E+00 & 3.13 E+00 \\ \text { BROWNS FERRY } & 2.18 E+01 & 2.42 E+01 \\ \text { BRUNSWICK } & 1.28 E+01 & 2.26 E+01 \\ \text { COOPER } & 8.77 E+00 & <8.37 E+00 \\ \text { DRESDEN 1 } & \text { N/R } & 0.00 E+00 \\ \text { DRESDEN 2-3 } & 6.20 E+01 & 6.05 E+00 \\ \text { DUANE ARNOLD } & \text { N/R } & 0.00 E+00 \\ \text { EDWIN 1. HATCH 1 } & 1.42 E+01 & 1.16 E+01 \\ \text { EDWIN I. HATCH 2 } & 1.07 E+01 & 9.28 E+00 \\ \text { HUMBOLDT BAY } & 9.70 E-02 & <1.62 E-01 \\ \text { JAMES A. FITZPATRICK } & 2.81 E+00 & 4.11 E+00 \\ \text { LACROSSE } & 7.20 E+01 & 7.74 E+01 \\ \text { MILLSTONE 1 } & 2.73 E+01 & 2.62 E+00 \\ \text { MONTICELLO } & 0.00 E+00 & 4.17 E-03 \\ \text { NINE MILE POINT } & \text { N/R } & 5.05 E+00 \\ \text { OYSTER CREEK } & 1.54 E+02 & 2.67 E+01 \\ \text { PEACH BOTTOM } & 3.73 E+01 & 3.68 E+01 \\ \text { PILGRIM } & 4.00 E+01 & 3.41 E+01 \\ \text { QUAD-CITIES } & 1.03 E+01 & 1.19 E+01 \\ \text { VERMONT YANKEE } & \text { N/R } & 3.70 E-01 \\ \text { FORT ST. VRAIN* } & 2.06 E+02 & 2.19 E+02\end{array}$


TABLE 6

LIQUID EFFLUENTS COMPARISON BY YEAR (CURIES)

TRITIUM

\section{PRESSURIZED WATER REACTORS}

\begin{tabular}{|c|c|c|c|c|c|c|c|c|c|c|}
\hline FACILITY & 1970 & 1971 & 1972 & 1973 & 1974 & 1975 & 1976 & 1977 & 1978 & 1979 \\
\hline ARKANSAS ONE 1 & & & & & $2.56 E+01$ & $4.60 \mathrm{E}+02$ & $2.12 E+02$ & $2.45 E+02$ & $2.94 E+02$ & $1.68 \mathrm{E}+02$ \\
\hline ARKANSAS ONE 2 & & & & & & & & & & $5.27 E+01$ \\
\hline BEAVER VALLEY & & & & & & & $8.60 E+00$ & $1.08 E+02$ & $3.49 E+02$ & $9.59 E+01$ \\
\hline CALVERT CLIFFS & & & & & & $2.63 E+02$ & $2.74 E+02$ & 5. $75 E+02$ & $4.56 E+02$ & 5. 14E+02 \\
\hline CRYSTAL RIVER & & & & & & & & $1.66 \mathrm{E}+02$ & $1.54 \mathrm{E}+02$ & $1.66 \mathrm{E}+02$ \\
\hline DAVIS-BESSE & & & & & & & & $9.01 \mathrm{E}+00$ & $2.15 \mathrm{E}+02$ & $2.45 E+02$ \\
\hline DONALD C. COOK & & & & & & $5.64 E+01$ & $1.92 E+02$ & $2.86 \mathrm{E}+02$ & $6.24 E+02$ & 1.22E+03 \\
\hline FORT CALHOUN & & & & $1.58 E+01$ & 1. $24 \mathrm{E}+02$ & $1.11 E+02$ & 1. $22 E+02$ & 1.57E+02 & 1.50E+02 & $2.58 \mathrm{E}+02$ \\
\hline H. B. ROBINSON & & 1. $18 \mathrm{E}+02$ & $4.05 E+02$ & 4.32E+02 & 4. 49E+02 & 6. 24E+02 & $9.80 E+02$ & $6.85 E+02$ & $4.73 E+02$ & $4.29 E+02$ \\
\hline HADDAM NECK & $7.40 E+03$ & $5.83 E+03$ & $5.89 \mathrm{E}+03$ & $3.90 E+03$ & $2.24 E+03$ & $5.67 E+03$ & $4.85 E+03$ & $6.67 E+03$ & $3.94 \mathrm{E}+03$ & $3.55 E+03$ \\
\hline INDIAN POINT 1-2 & & & & $2.75 E+01$ & $4.79 \mathrm{E}+01$ & $7.94 \mathrm{E}+01$ & 3.32E+02 & $3.71 \mathrm{E}+02$ & 5. 12E+02 & $3.75 E+02$ \\
\hline INDIAN POINT 3 & & & & & & & & & $2.56 \mathrm{E}+02$ & 1. $15 E+02$ \\
\hline JOSEPH M. FARLEY 1 & & & & & & & & & $5.91 \mathrm{E}+01$ & $9.40 E+01$ \\
\hline JOSEPH M. FARLEY 2 & & & & & & & & & & \\
\hline KEWAUNEE & & & & & $9.24 E+01$ & $2.77 \mathrm{E}+02$ & $1.80 \mathrm{E}+02$ & $2.95 \mathrm{E}+02$ & $2.96 \mathrm{E}+02$ & $2.49 E+02$ \\
\hline MAINE YANKEE & & & $9.20 E+00$ & $1.54 E+02$ & $2.19 E+02$ & $1.77 \mathrm{E}+02$ & 3.67E+02 & $1.53 \mathrm{E}+02$ & 3.15E+02 & $2.02 E+02$ \\
\hline MCGUIRE & & & & & & & & & & \\
\hline MILLSTONE 2 & & & & & & $7.60 \mathrm{E}+00$ & $2.77 E+02$ & $2.11 \mathrm{E}+02$ & $2.01 E+02$ & $2.54 E+02$ \\
\hline NORTH ANNA & & & & & & & & & $2.82 E+02$ & 3. 13E+02 \\
\hline OCONEE & & & & $7.07 E+01$ & 3.50E+02 & 3.55E+03 & $2.19 E+03$ & $1.92 E+03$ & 1. 17E+03 & $8.94 E+02$ \\
\hline PALISADES & & & $2.08 \mathrm{E}+02$ & $1.85 E+02$ & 8. $10 E+00$ & $4.16 E+01$ & $9.63 E+00$ & $5.58 E+01$ & $1.01 \mathrm{E}+02$ & $1.26 E+02$ \\
\hline POINT BEACH & & $2.66 \mathrm{E}+02$ & $5.63 E+02$ & $5.56 E+02$ & 8.33E+02 & $8.85 E+02$ & $6.94 E+02$ & $9.99 E+02$ & $1.29 \mathrm{E}+03$ & $8.92 \mathrm{E}+02$ \\
\hline PRAIRIE ISLAND & & & & $<1.00 \mathrm{E}-01$ & $1.42 E+02$ & $4.54 E-01$ & $1.00 \mathrm{E}-01$ & $1.35 E+03$ & $5.51 \mathrm{E}+02$ & $6.25 E+02$ \\
\hline R. E. GINNA & 1. $10 \mathrm{E}+02$ & 1. $.54 \mathrm{E}+02$ & 1. $19 E+02$ & $2.86 E+02$ & $1.95 E+02$ & $2.60 \mathrm{E}+02$ & $2.42 E+02$ & $1.19 \mathrm{E}+02$ & $2.42 E+02$ & $2.40 \mathrm{E}+02$ \\
\hline RANCHO SECO & & & & & & 1.32E+02 & $0.00 E+00$ & $8.55 \mathrm{E}-02$ & $\mathbf{N} / \mathbf{R}$ & $\mathbf{N} / \mathbf{R}$ \\
\hline SALEM 1 & & & & & & & $4.00 E-02$ & $2.96 E+02$ & $4.46 E+02$ & $7.26 \mathrm{E}+02$ \\
\hline SALEM 2 & & & & & & & & & & \\
\hline SAN ONOFRE & $4.80 E+03$ & 4. 57E+03 & $3.48 E+03$ & $4.07 E+03$ & $3.81 E+03$ & $4.00 E+03$ & $3.39 E+03$ & $1.79 E+03$ & $2.50 E+03$ & $2.32 E+03$ \\
\hline SEQUOYAH & & & & & & & & & & \\
\hline ST, LUCIE & & & & & & & $1.33 E+01$ & $2.42 E+02$ & $1.28 E+02$ & 1. 28E +02 \\
\hline SURRY & & & $5.00 E+00$ & 4.88E+02 & $2.45 E+02$ & $4.42 E+02$ & $7.82 E+02$ & $4.08 E+02$ & $7.47 E+02$ & 3. $57 \mathrm{E}+02$ \\
\hline THREE MILE ISLAND 1 & & & & & $1.30 E+02$ & $4.63 \mathrm{E}+02$ & $1.89 \mathrm{E}+02$ & $1.92 E+02$ & 1.55E+02 & 5.59E+01 \\
\hline $\begin{array}{l}\text { THREE MILE ISLAND } 2 \\
\text { TMI } 2 / \text { EPICOR }\end{array}$ & & & & & & & & & $3.83 \mathrm{E}+01$ & $7.81 \mathrm{E}+01$ \\
\hline TROJAN & & & & & & & $3.60 E+01$ & 3.11E+02 & $1.59 E+02$ & $6.80 \mathrm{E}+01$ \\
\hline TURKEY POINT & & & & 3. 29E+02 & 5.80E+02 & $7.97 E+02$ & $7.71 E+02$ & $9.24 E+02$ & $1.17 \mathrm{E}+03$ & $9.40 E+02$ \\
\hline YANKEE ROWE & $1.50 E+03$ & $1.68 \mathrm{E}+03$ & $8.03 E+02$ & $6.94 \mathrm{E}+02$ & 3. 14E+02 & $2.47 E+02$ & 1. $56 \mathrm{E}+02$ & 1. $39 \mathrm{E}+02$ & $1.96 E+02$ & $1.75 E+02$ \\
\hline ZION 1 & & & & $1.00 \mathrm{E}-01$ & $2.74 E+02$ & $1.03 E+03$ & $7.47 E+02$ & $7.24 E+02$ & $7.25 \mathrm{E}+02$ & $6.01 \mathrm{E}+02$ \\
\hline
\end{tabular}


TABLE 6

LIQUID EFFLUENTS COMPARISON BY YEAR (CURIES)

\author{
TRITIUM
}

PRESSURIZED WATER REACTORS

\begin{tabular}{|c|c|c|}
\hline FACILITY & 1980 & 1981 \\
\hline ARKANSAS ONE 1 & $2.12 \mathrm{E}+02$ & $4.42 E+02$ \\
\hline ARKANSAS ONE 2 & $2.89 E+02$ & $2.44 E+02$ \\
\hline BEAVER VALLEY & $3.98 \mathrm{E}+01$ & $1.40 \mathrm{E}+02$ \\
\hline CALVERT CLIFFS & +02 & \\
\hline CRYSTAL RIVER & 02 & E+02 \\
\hline DAVIS-BESSE & $.08 E+02$ & $1.57 \mathrm{E}+02$ \\
\hline DONALD C. COOK & $7.82 E+02$ & $9.15 \mathrm{E}+02$ \\
\hline FORT CALHOUN & $.44 \mathrm{E}+01$ & $2.42 E+02$ \\
\hline H. B. ROBINSON & $1.89 E+02$ & $1.86 \mathrm{E}+02$ \\
\hline HADDAM NECK & $.29 \mathrm{E}+03$ & $5.29 E+03$ \\
\hline INDIAN POINT 1-2 & $2.76 E+02$ & $2.41 \mathrm{E}+02$ \\
\hline INDIAN POINT 3 & $4.27 E+02$ & $6.42 \mathrm{E}+02$ \\
\hline JOSEPH M. FARLEY 1 & 5. $70 \mathrm{E}+02$ & $1.65 \mathrm{E}+02$ \\
\hline JOSEPH M. FARLEY 2 & & $6.34 \mathrm{E}+02$ \\
\hline REWAUNEE & $2.39 E+02$ & $2.51 \mathrm{E}+02$ \\
\hline MAINE YANKEE & $2.18 E+02$ & $2.16 \mathrm{E}+02$ \\
\hline MCGU IRE & & $6.25 \mathrm{E}+00$ \\
\hline MILLSTONE 2 & $E+02$ & 3. $71 \mathrm{E}+02$ \\
\hline NORTH ANNA & :02 & $1.28 \mathrm{E}+03$ \\
\hline OCONEE & t02 & $\mathbf{E}+02$ \\
\hline PAL I SADES & +01 & $E+02$ \\
\hline POINT BEACH & E+02 & $6.52 \mathrm{E}+02$ \\
\hline PRAIRIE ISLAND & $E+02$ & $8+02$ \\
\hline R. E. GINNA & +02 & $10 \mathrm{E}+02$ \\
\hline RANCHO SECO & -02 & E+01 \\
\hline SALEM 1 & $0.00 E+00$ & $3 \mathrm{E}+02$ \\
\hline SALEM 2 & N/R & 8.42E+02 \\
\hline SAN ONOFRE & $1.03 E+03$ & $2.97 E+02$ \\
\hline SEQUOYAH & $3.23 E-01$ & $7.65 \mathrm{E}+01$ \\
\hline ST. LUCIE & $2.72 E+02$ & 3. 25E+02 \\
\hline SURRY & $3.85 E+02$ & $5.31 \mathrm{E}+02$ \\
\hline THREE MILE ISLAND 1 & $3.26 E+01$ & $7.11 \mathrm{E}+00$ \\
\hline THREE MILE ISLAND 2 & $6.10 \mathrm{E}-04$ & 5.06E-02 \\
\hline TMI 2/EPICOR & $\mathbf{N} / \mathbf{R}$ & $0.00 \mathrm{E}+00$ \\
\hline TROJAN & $1.24 \mathrm{E}+02$ & $1.03 \mathrm{E}+02$ \\
\hline TURKEY POINT & $7.49 E+02$ & $1.95 \mathrm{E}+02$ \\
\hline YANKEE ROWE & $5.84 E+01$ & $1.03 E+02$ \\
\hline ZION 1 & $7.45 E+02$ & $6.04 E+02$ \\
\hline ZION & & $2.66 \mathrm{E}+02$ \\
\hline
\end{tabular}


TABLE 7

LIQUID EFFLUENTS COMPARISON BY YEAR (CURIES)

BOILING WATER REACTORS

BIG ROCK POINT

BROWNS FERRY

BRUNSWICK

COOPER

DRESDEN 1

DRESDEN 2-3

DUANE ARNOLD

EDWIN I . HATCH 1

EDWIN 1. HATCH 2

HUMBOLDT BAY

JAMES A. FITZPATR ICK

LACROSSE

MILLSTONE I

MONTI IELLO

NINE MILE POINT

OYSTER CREEK

PEACH BOTTOM

PILGR IM

QUAD-CITIES

VERMONT YANKEE

FORT ST. VRAIN*

MIXED FISSION AND ACTIVATION PRODUCTS

\begin{tabular}{|c|c|c|c|c|c|c|c|c|c|}
\hline 1970 & 1971 & 1972 & 1973 & 1974 & 1975 & 1976 & 1977 & 1978 & 1979 \\
\hline \multirow[t]{4}{*}{$4.70 \mathrm{E}+00$} & 3. $50 \mathrm{E}+00$ & 1. $10 \mathrm{E}+00$ & $2.70 \mathrm{E}+00$ & 1. $10 E+00$ & $2.02 E+00$ & $7.70 \mathrm{E}-01$ & $3.92 \mathrm{E}-01$ & $2.74 \mathrm{E}-01$ & $9.03 \mathrm{E}-01$ \\
\hline & & & & $8.00 \mathrm{E}-01$ & $2.70 \mathrm{E}+00$ & $<3.95 E+00$ & 1. $19 \mathrm{E}+00$ & $1.32 E+01$ & $1.02 \mathrm{E}+01$ \\
\hline & & & & & $1.89 E+00$ & $3.29 \mathrm{E}+00$ & $6.22 \mathrm{E}+00$ & $3.48 E+00$ & 5. 10E+00 \\
\hline & & & & 1. $40 \mathrm{E}+00$ & $1.74 \mathrm{E}+00$ & $7.00 \mathrm{E}-02$ & $7.50 \mathrm{E}-01$ & $3.05 E+00$ & $<2.48 E+00$ \\
\hline \multirow[t]{4}{*}{$8.20 \mathrm{E}+00$} & $6.20 E+00$ & $6.80 E+00$ & $9.20 E+00$ & $6.90 E+00$ & $8.40 E-01$ & $3.60 \mathrm{E}-01$ & $6.00 \mathrm{E}-01$ & $3.26 \mathrm{E}-01$ & $2.65 \mathrm{E}-02$ \\
\hline & $2.30 E+01$ & $2.20 \mathrm{E}+01$ & $2.59 \mathrm{E}+01$ & $3.31 \mathrm{E}+01$ & 8. 10E-01 & $1.21 \mathrm{E}+00$ & $4.40 \mathrm{E}-01$ & $3.99 \mathrm{E}-01$ & $2.65 \mathrm{E}-01$ \\
\hline & & & & & $<1.00 \mathrm{E}-02$ & $<1.00 \mathrm{E}-02$ & $2.32 \mathrm{E}-03$ & $2.73 \mathrm{E}-01$ & $5.10 \mathrm{E}-04$ \\
\hline & & & & & $6.00 \mathrm{E}-02$ & $4.00 \mathrm{E}-02$ & $2.50 \mathrm{E}+01$ & 4.03E-02 & $4.82 E-02$ \\
\hline \multirow[t]{2}{*}{$2.40 E+00$} & 1. $.80 \mathrm{E}+00$ & 1. $40 \mathrm{E}+00$ & $2.40 \mathrm{E}+00$ & $4.40 E+00$ & 3.79E+00 & $9.90 \mathrm{E}-01$ & $9.17 \mathrm{E}-01$ & $1.95 \mathrm{E}-01$ & $9.55 \mathrm{E}-02$ \\
\hline & & & & & $5.32 \mathrm{E}+00$ & $6.01 \mathrm{E}+00$ & $8.85 \mathrm{E}-01$ & $1.58 E+00$ & $6.46 \mathrm{E}-01$ \\
\hline \multirow[t]{3}{*}{$6.40 \mathrm{E}+00$} & $1.71 E+01$ & $4.85 E+01$ & 3.59E+01 & $1.31 \mathrm{E}+01$ & $1.42 E+01$ & $<5.78 E+00$ & $2.13 \mathrm{E}+01$ & $8.86 E+00$ & $1.67 E+00$ \\
\hline & $1.97 E+0 I$ & $5.15 E+01$ & 3. 34E+01 & 1. $.98 \mathrm{E}+02$ & 1. $99 \mathrm{E}+02$ & $9.65 \mathrm{E}+00$ & $5.27 \mathrm{E}-0 \mathrm{I}$ & $1.75 \mathrm{E}-01$ & $2.10 E-01$ \\
\hline & $<1.00 \mathrm{E}-01$ & $<1.00 \mathrm{E}-0 \mid$ & $0.00 E+00$ & $0.00 E+00$ & $0.00 E+00$ & $0.00 \mathrm{E}+00$ & $0.00 E+00$ & $0.00 E+00$ & $0.00 E+00$ \\
\hline $2.80 \mathrm{E}+01$ & 3. $22 E+01$ & $3.46 E+01$ & $4.08 \mathrm{E}+01$ & $2.56 \mathrm{E}+01$ & $2.10 E+01$ & $2.14 E+00$ & $3.03 \mathrm{E}-01$ & N/R & $1.89 \mathrm{E}+00$ \\
\hline \multirow[t]{6}{*}{$1.85 E+01$} & $1.20 \mathrm{E}+01$ & 1. $.00 E+01$ & $4.20 E+00$ & $7.00 \mathrm{E}-01$ & $4.10 E-01$ & $2.20 \mathrm{E}-01$ & $9.81 \mathrm{E}-02$ & 1.59E-02 & $6.59 \mathrm{E}-03$ \\
\hline & & & $\langle 1.00 \mathrm{E}-0|$ & $9.00 \mathrm{E}-01$ & $9.30 \mathrm{E}-01$ & $3.38 E+00$ & $2.23 E+00$ & $5.11 \mathrm{E}+00$ & 1. $95 \mathrm{E}+01$ \\
\hline & & 1. $50 \mathrm{E}+00$ & $9.00 \mathrm{E}-01$ & $4.20 E+00$ & $8.01 E+00$ & $2.33 E+00$ & $3.41 E+00$ & 1. $.77 E+00$ & 5. 12E-01 \\
\hline & & $2.40 \mathrm{E}+00$ & $2.14 E+01$ & $3.88 E+01$ & $1.71 \mathrm{E}+01$ & $6.99 \mathrm{E}+00$ & 1. $34 \mathrm{E}+00$ & $2.24 \mathrm{E}+00$ & $1.31 E+00$ \\
\hline & & & $<1.00 \mathrm{E}-01$ & $0.00 \mathrm{E}+00$ & $<1.00 \mathrm{E}-02$ & $<1.00 \mathrm{E}-02$ & $1.55 \mathrm{E}-01$ & N/R & $2.40 \mathrm{E}-04$ \\
\hline & & & & & & & & & $1.89 \mathrm{E}-04$ \\
\hline
\end{tabular}


TABLE 7

LIQUID EFFLUENTS COMPARISON BY YEAR (CURIES)

BOILING WATER REACTORS

\begin{tabular}{|c|c|c|}
\hline FACILITY & 1980 & 1981 \\
\hline BIG ROCK POINT & $7.82 \mathrm{E}-01$ & $3.91 \mathrm{E}-01$ \\
\hline BROWNS FERRY & $9.38 E+00$ & $2.24 E+00$ \\
\hline BRUNSW I CK & $1.26 \mathrm{E}+00$ & $2.20 E+00$ \\
\hline COOPER & $<1.10 E+01$ & $<3.61 E+00$ \\
\hline DRESDEN 1 & $\mathbf{N} / \mathbf{R}$ & $0.00 E+00$ \\
\hline DRESDEN 2-3 & $7.16 \mathrm{E}-01$ & $6.12 \mathrm{E}-02$ \\
\hline DUANE ARNOLD & $\mathbf{N} / \mathbf{R}$ & $0.00 E+00$ \\
\hline EDWIN I HATCH 1 & $6.83 \mathrm{E}-02$ & $3.73 E-01$ \\
\hline EDWIN I, HATCH 2 & 4.57E-02 & $1.63 \mathrm{E}-01$ \\
\hline HUMBOLDT BAY & 1.39E-01 & 1. $55 \mathrm{E}-01$ \\
\hline JAMES A. FITZPATRICK & 1. $51 \mathrm{E}+00$ & $2.51 \mathrm{E}+00$ \\
\hline LACROSSE & $2.13 \mathrm{E}+00$ & $2.26 \mathrm{E}-01$ \\
\hline MILLSTONE 1 & $7.24 \mathrm{E}-01$ & 3.94E-01 \\
\hline MONTICELLO & $0.00 E+00$ & $3.11 \mathrm{E}-06$ \\
\hline NINE MILE POINT & $\mathbf{N} / \mathbf{R}$ & $5.35 E+00$ \\
\hline OYSTER CREEK & $5.06 \mathrm{E}-01$ & $2.48 E-01$ \\
\hline РEACH ВОТTOM & 1. $.90 E+00$ & $1.97 E+00$ \\
\hline PILGRIM & $2.73 E+00$ & 1. $94 E+00$ \\
\hline QUAD-CITIES & $1.31 \mathrm{E}+01$ & 3.27E+00 \\
\hline VERMONT YANKEE & N/R & 1. $02 E-02$ \\
\hline FORT ST, VRAIN* & $6.37 E-05$ & $3.64 E-04$ \\
\hline
\end{tabular}

MIXED FISSION AND ACTIVATION PRODUCTS 
TABLE 8

LIQUID EFFLUENTS COMPARISON BY YEAR (CURIES)

\section{PRESSURIZED WATER REACTORS}

\section{FACILITY}

ARKANSAS ONE 1 ARKANSAS ONE 2 BEAVER VALLEY

CALVERT CLIFFS

CRYSTAL RIVER

DAVIS-BESSE

DONALD C. COOK

FORT CALHOUN

H. B, ROBINSON

HADDAM NECK

INDIAN POINT 1-2

INDIAN POINT 3

JOSEPH M. FARLEY

JOSEPH $M$. FARLEY 2

KEWAUNEE

MAINE YANKEE

MCGUIRE

MILLSTONE 2

NORTH ANNA

OCONEE

PALI SADES

POINT BEACH

PRAIRIE ISLAND

R. E. GINNA

RANCHO SECO

SALEM 1

SALEM 2

SAN ONOFRE

SEQUOYAH

ST. LUCIE

SURRY

THREE MILE ISLAND

THREE MILE ISLAND 2

TMI 2/EPICOR

TROJAN

TURKEY POINT

YANKEE ROWE

ZION 1

ZION 2

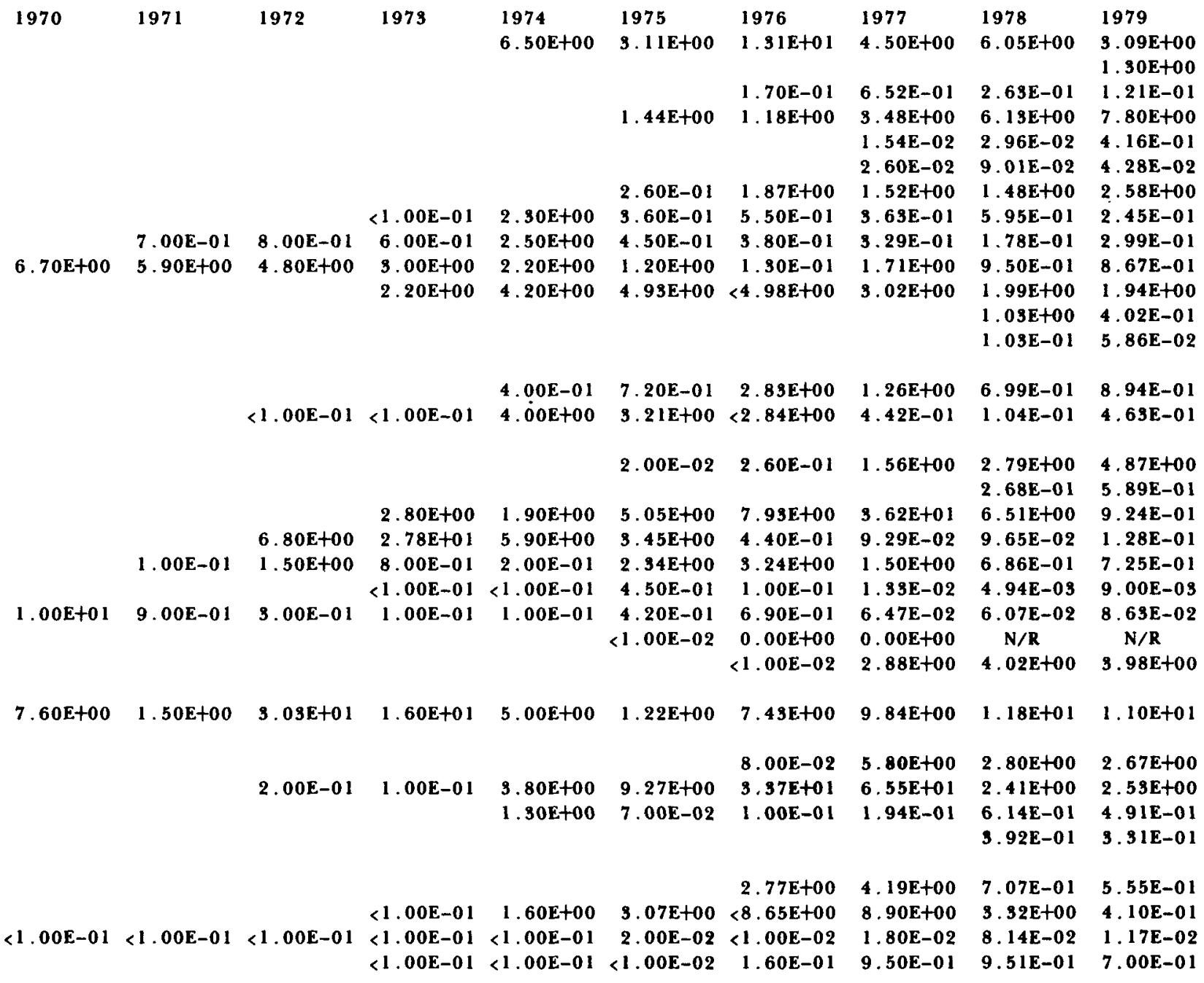


TABLE 8

LIQUID EFFLUENTS COMPARISON BY YEAR (CURIES)

\section{PRESSURIZED WATER REACTORS}

\begin{tabular}{llll} 
FACILITY & 1980 & 1981 \\
ARKANSAS ONE 1 & $3.42 E+00$ & $7.50 E+00$ \\
ARKANSAS ONE 2 & $4.13 E+00$ & $2.95 E+00$ \\
BEAVER VALLEY & $1.04 E-01$ & $1.44 E-01$ \\
CALVERT CLIFFS & $4.53 E+00$ & $2.68 E+00$ \\
CRYSTAL RIVER & $1.46 E-01$ & $1.29 E-01$ \\
DAVIS-BESSE & $2.07 E-01$ & $7.92 E-01$ \\
DONALD C. COOK & $1.37 E+00$ & $1.86 E+00$ \\
FORT CALHOUN & $5.04 E-01$ & $1.65 E-01$ \\
H. B. ROBINSON & $3.58 E-01$ & $1.84 E+00$ \\
HADDAM NECK & $2.76 E-01$ & $7.12 E-01$ \\
INDIAN POINT 1-2 & $1.26 E+00$ & $5.67 E+00$ \\
INDIAN POINT 3 & $2.90 E+00$ & $2.62 E+00$ \\
JOSEPH M. FARLEY 1 & $6.18 E-02$ & $1.31 E-01$ \\
JOSEPH M. FARLEY 2 & & $2.69 E-02$ \\
KEWAUNEE & $6.17 E-01$ & $8.15 E-01$ \\
MAINE YANKEE & $2.97 E-01$ & $4.36 E-01$ \\
MCGUIRE & & $3.94 E-01$ \\
MILLSTONE 2 & $2.81 E+00$ & $4.18 E+00$ \\
NORTH ANNA & $1.05 E+00$ & $6.76 E-01$ \\
OCONEE & $1.54 E+00$ & $1.75 E+00$ \\
PALISADES & $8.73 E-03$ & $3.31 E-02$ \\
POINT BEACH & $6.29 E-01$ & $1.01 E+00$ \\
PRAIRIE ISLAND & $1.32 E-02$ & $9.12 E-03$ \\
R. E. GINNA & $1.96 E-02$ & $3.85 E-02$ \\
RANCHO SECO & $3.78 E-03$ & $5.92 E-01$ \\
SALEM 1 & $2.65 E+00$ & $2.80 E+00$ \\
SALEM 2 & $3.89 E-01$ & $1.51 E+00$ \\
SAN ONOFRE & $1.12 E+01$ & $3.64 E+00$ \\
SEQUOYAH & N/R & $2.76 E+00$ \\
ST. LUCIE & $2.36 E+00$ & $2.46 E+00$ \\
SURRY & $3.85 E+00$ & $6.11 E+00$ \\
THREE MILE ISLAND 1 & $1.83 E-01$ & $8.69 E-02$ \\
THREE MILE ISLAND 2 & $1.45 E-05$ & $2.22 E-05$ \\
TMI 2/EPICOR & N/R & $0.00 E+00$ \\
TROJAN & $7.87 E-01$ & $9.94 E-01$ \\
TURKEY POINT & $6.78 E-01$ & $3.03 E-01$ \\
YANKEE ROWE & $1.75 E-02$ & $1.43 E-02$ \\
ZION 1 & $4.74 E-01$ & $1.61 E+00$ \\
ZION 2 & & $1.05 E+00$ \\
& & \\
\hline
\end{tabular}
$3.42 \mathrm{E}+00 \quad 7.50 \mathrm{E}+00$
$4.13 \mathrm{E}+00 \quad 2.95 \mathrm{E}+00$
$\begin{array}{ll}1.04 \mathrm{E}-01 & 1.44 \mathrm{E}-01 \\ 4.53 \mathrm{E}+00 & 2.68 \mathrm{E}+00\end{array}$
$7.92 \mathrm{E}-01$
$5.67 \mathrm{E}+00$
$\begin{array}{ll}2.90 \mathrm{E}+00 & 2.62 \mathrm{E}+00 \\ 6.18 \mathrm{E}-02 & 1.31 \mathrm{E}-01\end{array}$
- 01
$8 E+00$
$6.76 E-01$
54E+00 1.75E+00
$6.29 \mathrm{E}-01 \quad 1.01 \mathrm{E}+00$
32E-02 $9.12 \mathrm{E}-09$
96E-02 3.85E-02
$2.65 \mathrm{E}+00 \quad 2.80 \mathrm{E}+00$
$1.51 \mathrm{E}+00$
$12 E+01$ 3.64E+00
2.76E+00
$0.00 E+00$
$6.78 E-013.03 E-01$
$1.05 E+00$

MIXED FISSION AND ACTIVATION PRODUCTS 
TABLE 9

BOILING WATER REACTORS

FACI IITY

BIG ROCK POINT

BROWNS FERRY

BRUNSWI CK

COOPER

DRESDEN

DUANE ARNOLD

EDWIN I . HATCH 1

EDWIN I HATCH 2

HUMBOLDT BAY

JAMES A. FITZPATR ICK

LACROSSE

MILLSTONE 1

MONTICELLO

NINE MILE POINT

OYSTER CREEK

PEACH BOTTOM

PILGRIM

QUAD-CITIES

VERMONT YANKEE

FORT ST. VRAIN*
SOLID WASTE SUMMARY 1981

VOLUME
(CUB IC METERS)
1.44E+02
N/R
$4.30 E+03$
$4.99 E+02$
$1.14 E+03$
$6.97 E+02$
$1.29 E+03$
$1.40 E+03$
$8.43 E+01$
$8.61 E+02$
$4.82 E+00$
$1.96 E+03$
$5.54 E+02$
$5.31 E+02$
$1.78 E+03$
$2.34 E+03$
$1.06 E+03$
$1.72 E+03$
$4.39 E+02$
$0.00 E+00$

3.17E+02$$
N / R
$$

7.47E+03

4. . 43E+02

$4.59 E+03$

$1.07 E+03$

4. $46 E+03$

3.05E+02

5. 46E-01

1. $63 \mathrm{E}+03$

$6.11 E+01$

1. 82E+03

4. 42E+02

1. 72E+03

$4.21 \mathrm{E}+02$

5. 33E+03

5. 16E+03

1. $11 \mathrm{E}+03$

$0.00 E+00$
No. OF

SHI PMENTS

14
$N / R$
374
74
230
118
128
92
3
102
2
129
52
76
156
335
78
335
44
0


TABLE 10

PRESSURIZED WATER REACTORS

FACILITY

ARKANSAS ONE 1

ARKANSAS ONE

BEAVER VALLEY

CALVERT CLIFFS

CRYSTAL RIVER

DAVIS-BESSE

DONALD C. COOK

FORT GALHOUN

H. B. ROBINSON

HADDAM NECK

INDIAN POINT 1-2

INDIAN POINT 9

JOSEPH $M$. FARLEY

KEWAUNEE

MAINE YANKEE

MCGUIRE

MILLSTONE 2

NORTH ANNA

OCONEE

PALISADES

POINT BEACH

PRAIRIE ISLAND

R. E. GINNA

RANCHO SECO

SALEM $1 \& 2$

SAN ONOFRE

SEQUOYAH

ST. LUCIE

SURRY

THREE MILE ISLAND

THREE MILE ISLAND 2

TMI 2/EPICO

TROJAN

TURKEY POINT

YANKEE ROWE

ZION
SOLID WASTE SUMMARY 1981

VOLUME

ACTIVITY

$\mathbf{N} / \mathrm{R}$

N/R

9. $30 \mathrm{E}+01$

9. 86E+01

1. 38E+03

3. $95 \mathrm{E}+01$

$1.43 \mathrm{E}+03$

1. $01 \mathrm{E}+02$

1. $88 \mathrm{E}+01$

$6.61 \mathrm{E}+02$

$1.71 \mathrm{E}+03$

6. 40E+01

7. 20E+02

1. $98 \mathrm{E}+02$

1. $67 \mathrm{E}+03$

1. $31 \mathrm{E}-01$

3. $21 \mathrm{E}+02$

2.62E+03

1. $12 \mathrm{E}+04$

1. $57 \mathrm{E}+04$

4. 87E+0

5. $64 \mathrm{E}+01$

$6.35 \mathrm{E}+02$

1. $44 \mathrm{E}+02$

1. $14 \mathrm{E}+03$

1. $26 \mathrm{E}+03$

2. $92 \mathrm{E}+01$

2.96E+02

1. 36E+03

2.34E+02

5. 11E+01

3. $50 \mathrm{E}+02$

1. 04E+03

1. $17 \mathrm{E}+02$

6. 79E+01

3. 44 E+03

$.51 \mathrm{E}+02$

. 75E+02

3. 08E+02

1. $53 \mathrm{E}+03$
NO. OF

SH I PMENTS

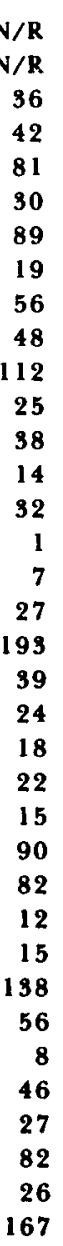


TABLE 11

BOILING WATER REACTORS

FACILITY

BIG ROCK POINT

BROWNS FERRY

BRUNSWICK

COOPER

DRESDEN

DUANE ARNOLD

EDWIN 1 . HATCH 1

EDWIN 1. HATCH 2

HUMBOLDT BAY

JAMES A. FITZPATR ICK

LACROSSE

MILLSTONE 1

MONTICELLO

NINE MILE POINT

OYSTER CREEK

PEACH BOTTOM

PILGRIM

QUAD-CITIES

VERMONT YANKEE

FORT ST. VRAIN*

SOLID WASTE COMPARISON BY YEAR

VOLUME (CUB IC METERS) - ACTIVITY (CUR IES)

1975

1976

$\begin{array}{rlllllll}2.88 E+01 & 3.69 E+00 & 7.22 E+01 & 9.68 E+02 & 3.10 E+01 & 2.56 E+01 & 8.99 E+01 & 2.77 E+02 \\ 3.20 E+02 & 1.03 E+02 & 1.82 E+03 & 1.10 E+04 & 2.90 E+03 & 1.33 E+03 & 2.29 E+03 & 4.17 E+03 \\ 1.79 E+03 & 6.46 E+02 & 2.47 E+03 & 3.24 E+03 & 2.02 E+03 & 2.14 E+03 & 3.09 E+03 & 4.29 E+03 \\ 3.01 E+02 & 3.20 E+02 & 2.83 E+02 & 2.85 E+02 & 3.29 E+02 & 3.84 E+02 & 5.65 E+02 & 9.69 E+01 \\ 7.12 E+03 & 4.33 E+03 & 2.25 E+03 & 1.13 E+04 & 1.77 E+03 & 1.88 E+03 & 1.04 E+03 & 8.45 E+02 \\ 5.95 E+02 & 1.87 E+02 & 5.45 E+02 & 4.98 E+02 & 1.10 E+03 & 1.86 E+03 & 7.99 E+02 & 8.01 E+02 \\ 4.12 E+02 & 2.91 E+02 & 5.39 E+02 & 3.81 E+02 & 7.50 E+02 & 1.09 E+04 & 9.78 E+02 & 2.70 E+02 \\ & & & & & & & \\ 8.49 E+01 & 4.09 E+00 & 3.77 E+02 & 2.00 E+01 & 1.78 E+02 & 7.91 E-01 & 9.06 E+01 & 3.35 E+03 \\ 6.19 E+02 & 3.41 E+02 & 1.23 E+03 & 6.17 E+03 & 8.70 E+02 & 3.19 E+02 & 8.04 E+02 & 1.06 E+03 \\ N / R & N / R & 4.65 E+00 & 5.88 E+02 & 3.80 E+01 & 6.18 E+01 & 5.09 E+00 & 1.25 E+02 \\ 1.33 E+03 & 1.70 E+03 & 1.77 E+03 & 3.03 E+03 & 2.00 E+03 & 8.15 E+04 & 2.11 E+03 & 1.16 E+03 \\ 2.85 E+02 & 3.79 E+03 & 5.73 E+02 & 2.91 E+04 & 4.99 E+02 & 6.35 E+04 & 4.74 E+02 & 1.31 E+04 \\ 5.38 E+02 & 2.51 E+03 & 6.65 E+02 & 2.51 E+04 & 3.85 E+02 & 2.24 E+04 & 4.97 E+02 & 1.52 E+03 \\ 1.20 E+03 & 1.29 E+03 & 1.74 E+03 & 2.73 E+02 & 1.54 E+03 & 1.15 E+03 & 1.13 E+03 & 1.34 E+03 \\ 1.20 E+03 & 5.85 E+02 & 2.52 E+03 & 1.82 E+03 & 1.96 E+03 & 4.97 E+03 & 2.40 E+03 & 8.03 E+03 \\ 9.12 E+02 & 3.69 E+04 & 5.84 E+02 & 5.70 E+03 & 1.97 E+03 & 4.92 E+04 & 3.03 E+03 & 2.22 E+04 \\ 1.00 E+03 & 2.35 E+03 & 1.20 E+03 & 7.53 E+03 & 1.34 E+03 & 3.27 E+03 & 7.82 E+02 & 4.26 E+03 \\ 2.38 E+02 & 2.93 E+01 & 1.08 E+02 & 1.76 E+02 & 3.99 E+02 & 5.39 E+04 & 2.71 E+02 & 9.99 E+02 \\ & & & & & & 0.00 E+00 & 0.00 E+00\end{array}$


TABLE, 11

SOLID WASTE COMPARISON BY YEAR

BOILING WATER REACTORS

FACILITY

BIG ROCK POINT

BROWNS FERRY

BRUNSWICK

COOPER

DRESDEN

DUANE ARNOLD

EDWIN 1. HATCH 1

EDWIN 1. HATCH 2

HUMBOLDT BAY

JAMES A. FITZPATRICK

LACROSSE

MILLSTONE 1

MONTICELLO

NINE MILE POINT

OYSTER CREEK

PEACH BOTTOM

PILGR IM

QUAD-CITIES

VERMONT YANKEE

FORT ST. VRAIN
VOLUME(CUBIC METERS) - ACTIVITY(CURIES)

1980

$\begin{array}{llll}4.20 E+01 & 3.09 E+01 & 1.44 E+02 & 3.17 E+02 \\ 2.49 E+03 & 6.46 E+03 & N / R & N / R \\ 6.73 E+03 & 7.55 E+03 & 4.30 E+03 & 7.47 E+03 \\ 4.35 E+02 & 7.05 E+02 & 4.99 E+02 & 4.43 E+02 \\ 1.16 E+03 & 4.46 E+03 & 1.14 E+03 & 4.59 E+03 \\ 7.35 E+02 & 7.00 E+02 & 6.97 E+02 & 1.07 E+03 \\ 4.64 E+02 & 9.62 E+02 & 1.29 E+03 & 4.46 E+03 \\ 2.59 E+02 & 8.27 E+01 & 1.40 E+03 & 3.05 E+02 \\ 8.20 E+01 & 6.95 E+01 & 8.43 E+01 & 5.46 E-01 \\ 7.50 E+02 & 8.86 E+02 & 8.61 E+02 & 1.63 E+03 \\ 4.32 E+01 & 2.02 E+01 & 4.82 E+00 & 6.11 E+01 \\ 2.30 E+03 & 2.36 E+03 & 1.96 E+03 & 1.82 E+03 \\ 7.42 E+02 & 7.57 E+02 & 5.54 E+02 & 4.42 E+02 \\ 8.14 E+02 & 2.32 E+04 & 5.31 E+02 & 1.72 E+03 \\ 2.03 E+03 & 1.32 E+03 & 1.78 E+03 & 4.21 E+02 \\ 2.64 E+03 & 6.69 E+03 & 2.34 E+03 & 5.33 E+03 \\ 2.94 E+03 & 1.60 E+03 & 1.06 E+03 & 9.38 E+02 \\ 1.67 E+03 & 4.07 E+03 & 1.72 E+03 & 5.16 E+03 \\ 4.84 E+02 & 9.20 E+02 & 4.39 E+02 & 1.11 E+03 \\ 0.00 E+00 & 0.00 E+00 & 0.00 E+00 & 0.00 E+00\end{array}$


TABLE 12

SOLID WASTE COMPARISON BY YEAR

PRESSURIZED WATER REACTORS

FACILITY

ARKANSAS ONE 1

ARKANSAS ONE 2

BEAVER VALLEY

CALVERT CLIFFS

CRYSTAL RIVER

DAVIS-BESSE

DONALD C. COOK

FORT CALHOUN

H. B. ROBINSON

HADDAM NECK

INDIAN POINT 1-2

INDIAN POINT 3

JOSEPH M. FARLEY

KEWAUNEE

MAINE YANKEE

MCGUIRE

MILLSTONE 2

NORTH ANNA

OCONEE

PALISADES

POINT BEACH

PRAIRIE ISLAND

R. E. GINNA

RANCHO SECO

SALEM 182

SAN ONOFRE

SEQUOYAH

ST. LUCIE

SURRY

THREE MILE I SLAND I

THREE MILE ISLAND 2

TMI 2/EPICOR

TROJAN

TURKEY POINT

YANKEE ROWE

ZION
1975

VOLUME (CUB IC METERS) - ACTIVITY(CURIES)

\begin{tabular}{|c|c|c|c|c|c|c|c|}
\hline \multicolumn{2}{|c|}{1976} & \multicolumn{2}{|c|}{1977} & \multicolumn{2}{|c|}{1978} & \multicolumn{2}{|c|}{1979} \\
\hline $\mathbf{N} / \mathbf{R}$ & $\mathbf{N} / \mathbf{R}$ & 3. $17 E+02$ & 1. $26 \mathrm{E}+02$ & $\mathbf{N} / \mathbf{R}$ & $\mathbf{N} / \mathbf{R}$ & N/R & $\mathbf{N} / \mathbf{R}$ \\
\hline & & & & & & $N / R$ & $\mathbf{N} / \mathbf{R}$ \\
\hline $4.30 \mathrm{E}+01$ & 4.26E-02 & $2.67 \mathrm{E}+02$ & 8. 18E+00 & $4.39 E+02$ & $2.25 E+02$ & $2.44 E+02$ & $2.95 \mathrm{E}+02$ \\
\hline $1.18 E+02$ & $1.22 \mathrm{E}+02$ & 3.09E+02 & $9.83 E+02$ & $6.03 E+02$ & 1. $12 \mathrm{E}+03$ & $4.32 E+02$ & $9.71 E+02$ \\
\hline & & $4.48 E+02$ & $3.48 \mathrm{E}+00$ & $6.87 E+02$ & $2.72 E+04$ & 1. $24 \mathrm{E}+03$ & 1. $20 \mathrm{E}+03$ \\
\hline & & $0.00 \mathrm{E}+00$ & $0.00 E+00$ & 3. $40 \mathrm{E}+02$ & 3. $30 \mathrm{E}+00$ & $2.60 \mathrm{E}+02$ & $2.86 \mathrm{E}+00$ \\
\hline $1.69 \mathrm{E}+02$ & $2.62 \mathrm{E}-01$ & $6.84 E+02$ & $8.28 E+01$ & 1. $28 \mathrm{E}+03$ & $2.25 E+02$ & 1. $09 \mathrm{E}+03$ & $3.37 \mathrm{E}+02$ \\
\hline $7.57 E+02$ & $1.27 E+02$ & 5.97E+02 & $6.46 \mathrm{E}+02$ & $5.84 E+02$ & 1.06E+02 & $2.44 E+02$ & $2.99 E+01$ \\
\hline $3.16 E+02$ & $6.29 \mathrm{E}+01$ & $2.59 \mathrm{E}+02$ & $1.24 E+03$ & $8.22 E+02$ & $2.40 E+02$ & $8.34 \mathrm{E}+02$ & $8.72 E+01$ \\
\hline $7.67 E+02$ & $7.46 \mathrm{E}+02$ & $1.68 \mathrm{E}+03$ & $8.41 \mathrm{E}+02$ & 2. 29E +02 & $1.44 E+02$ & 1. $29 E+03$ & $3.05 E+02$ \\
\hline $19 \mathrm{E}+02$ & $9.46 \mathrm{E}+02$ & $1.06 \mathrm{E}+03$ & $1.45 E+03$ & $8.43 E+03$ & $2.37 E+03$ & $1.17 E+03$ & $2.16 E+03$ \\
\hline & & & & $5.94 E+02$ & $6.49 E+01$ & $2.25 \mathrm{E}+02$ & 1.63E+02 \\
\hline & & & & $2.69 \mathrm{E}+02$ & $5.72 E+00$ & $1.11 \mathrm{E}+03$ & $2.32 E+02$ \\
\hline $.94 E+02$ & $4.94 E+01$ & 3.37E+01 & $3.66 \mathrm{E}+02$ & $7.98 E+01$ & 1. $50 \mathrm{E}+03$ & $1.70 E+02$ & $3.54 E+02$ \\
\hline $.84 E+02$ & $5.04 \mathrm{E}+02$ & 1.84E+02 & $1.53 E+04$ & 5.81E+02 & 4. $14 E+03$ & 3.63E+02 & $2.77 E+03$ \\
\hline $2.80 \mathrm{E}+02$ & $1.80 \mathrm{E}+00$ & $9.35 E+01$ & $5.80 \mathrm{E}+01$ & 1. $55 \mathrm{E}+02$ & $1.70 \mathrm{E}+01$ & $2.46 \mathrm{E}+02$ & $1.78 E+09$ \\
\hline & & & & $2.14 E+01$ & 3. $59 \mathrm{E}+00$ & $2.95 \mathrm{E}+02$ & $5.89 E+01$ \\
\hline $.22 E+03$ & 7.83E+02 & $1.07 E+03$ & $7.37 E+03$ & $1.58 E+03$ & $5.93 E+03$ & $1.63 E+03$ & $2.59 E+03$ \\
\hline $6.81 \mathrm{E}+01$ & 9.58E+01 & $4.43 E+02$ & $8.71 \mathrm{E}+01$ & $7.17 E+02$ & 3. $40 \mathrm{E}+03$ & $6.84 E+02$ & 3.92E+02 \\
\hline 1. $.99 \mathrm{E}+02$ & $3.04 \mathrm{E}+02$ & $6.84 E+03$ & 5.68E+02 & $1.61 E+02$ & 1. $51 E+03$ & $2.69 E+02$ & 1. $22 \mathrm{E}+03$ \\
\hline $1.52 \mathrm{E}+02$ & $6.53 E+01$ & $6.43 E+02$ & $2.46 \mathrm{E}+02$ & 1. $95 \mathrm{E}+02$ & 1. $53 \mathrm{E}+02$ & $1.99 E+01$ & $8.83 E+01$ \\
\hline $2.80 E+02$ & $9.78 E+01$ & $3.49 E+02$ & $6.90 \mathrm{E}+02$ & $5.96 \mathrm{E}+01$ & $6.27 \mathrm{E}+02$ & $3.08 \mathrm{E}+02$ & $1.53 E+02$ \\
\hline $1.11 \mathrm{E}+02$ & $2.80 \mathrm{E}+01$ & $5.06 \mathrm{E}+01$ & $1.21 \mathrm{E}+03$ & 1. $29 E+02$ & 1. $27 E+03$ & $1.01 \mathrm{E}+02$ & $4.03 E+00$ \\
\hline $0.00 \mathrm{E}+00$ & $0.00 E+00$ & 4.25E+02 & $2.20 \mathrm{E}+00$ & 2. 27E+02 & 1.94E+02 & $6.86 E+02$ & 1. $28 \mathrm{E}+02$ \\
\hline $1.45 E+02$ & $6.98 \mathrm{E}+02$ & 3.68E+02 & $6.02 E+01$ & $1.31 E+02$ & $7.17 E+00$ & $8.35 \mathrm{E}+01$ & $9.24 \mathrm{E}+01$ \\
\hline $8.68 E+01$ & $1.57 \mathrm{E}+00$ & $85 E+02$ & $.27 \mathrm{E}+03$ & $3.58 \mathrm{E}+02$ & $1.26 \mathrm{E}+04$ & 3.08E+02 & 1. $.79 \mathrm{E}+02$ \\
\hline $7.00 E+02$ & $6.17 E+02$ & $7.93 E+02$ & $6.10 E+02$ & $6.03 E+02$ & $5.66 \mathrm{E}+02$ & $2.74 E+03$ & 3. 45E+02 \\
\hline 4.06E+02 & $1.85 E+02$ & 2. 18E+02 & $4.73 E+01$ & 3.89E+02 & $2.34 E+02$ & $7.51 \mathrm{E}+02$ & 3. $12 E+01$ \\
\hline $4.37 E+01$ & $4.32 E+00$ & $1.01 \mathrm{E}+02$ & $8.31 \mathrm{E}+01$ & $2.26 \mathrm{E}+02$ & $4.48 E+02$ & $6.37 E+02$ & $3.30 E+02$ \\
\hline $1,44 E+03$ & 4.77E+02 & $1.07 E+03$ & $4.26 E+02$ & $1.75 E+03$ & $1.72 E+03$ & $9.20 \mathrm{E}+02$ & $2.48 E+02$ \\
\hline 3.60E+02 & $2.65 \mathrm{E}+01$ & $2.81 E+02$ & 3. $54 \mathrm{E}+00$ & $2.60 E+02$ & $9.75 E+00$ & $2.36 E+02$ & $1.63 \mathrm{E}+02$ \\
\hline
\end{tabular}

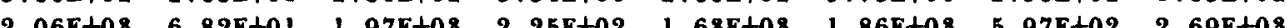


TABLE 12

SOLID WASTE COMPARISON BY YEAR

PRESSURIZED WATER REACTORS

VOLUME (CUBIC METERS) - ACTIVITY(CURIES)

\begin{tabular}{|c|c|c|c|c|}
\hline FACILITY & 198 & & 198 & \\
\hline ARRANSAS ONE 1 & $\mathbf{N} / \mathbf{R}$ & $\mathbf{N} / \mathbf{R}$ & $\mathbf{N} / \mathbf{R}$ & $N / R$ \\
\hline ARKANSAS ONE 2 & $\mathbf{N} / \mathbf{R}$ & $N / R$ & $\mathbf{N} / \mathbf{R}$ & $N / R$ \\
\hline BEAVER VALLEY & $2.84 \mathrm{E}+02$ & 5.34E+02 & 2. 13E+02 & 9.30E+01 \\
\hline CALVERT CLIFFS & $2.51 \mathrm{E}+02$ & $1.48 E+04$ & 5.00E +02 & $9.86 \mathrm{E}+01$ \\
\hline CRYSTAL RIVER & $9.27 \mathrm{E}+02$ & $2.05 E+03$ & 1. $27 E+03$ & $1.38 \mathrm{E}+03$ \\
\hline DAVIS-BESSE & 3.30E+02 & 3.00E+01 & 3. $25 \mathrm{E}+02$ & $3.95 \mathrm{E}+01$ \\
\hline DONALD C. COOK & 2. $10 \mathrm{E}+03$ & $1.04 \mathrm{E}+03$ & $9.63 \mathrm{E}+02$ & $1.43 \mathrm{E}+03$ \\
\hline FORT CALHOUN & $4.06 \mathrm{E}+02$ & 1. $32 E+03$ & $2.53 E+02$ & $1.01 \mathrm{E}+02$ \\
\hline H. B. ROBIINSON & $3.99 E+03$ & 3.08E+02 & $9.02 E+02$ & 1. 88E+01 \\
\hline HADDAM NECK & $1.26 E+03$ & $4.89 E+02$ & $4.38 E+02$ & $6.61 E+02$ \\
\hline INDIAN POINT 1-2 & $1.03 E+03$ & 3. $32 E+02$ & $1.58 E+03$ & $1.71 \mathrm{E}+0$ \\
\hline INDIAN POINT 3 & 3.47E+02 & $2.02 E+02$ & 3. $17 \mathrm{E}+02$ & $6.40 E+01$ \\
\hline JOSEPH $M$. FARLEY & $4.41 E+02$ & $2.26 E+02$ & 5.64E+02 & $7.20 E+02$ \\
\hline KEWAUNEE & $1.03 E+02$ & $1.37 E+03$ & $7.38 E+01$ & $1.98 \mathrm{E}+02$ \\
\hline MAINE YANKEE & $4.57 \mathrm{E}+02$ & 4.79E+03 & 4. 14E+02 & $1.67 \mathrm{E}+03$ \\
\hline MCGUIRE & & & $1.98 E+01$ & $1.31 \mathrm{E}-01$ \\
\hline MILLSTONE 2 & $7.51 \mathrm{E}+00$ & $2.28 \mathrm{E}+02$ & $1.63 E+01$ & $3.21 \mathrm{E}+02$ \\
\hline NORTH ANNA & 2.6 & 1.5 & 3.0 & 2.6 \\
\hline OCONEE & $1.32 E+03$ & 2.9 & 2.48 & 1. $12 E+04$ \\
\hline PALI I SADES & $7.31 E+02$ & 1.18 & 8.54 & I. $57 \mathrm{E}+04$ \\
\hline POINT BEACH & $4.49 \mathrm{E}+02$ & 9.3 & 1.77 & $4.87 \mathrm{E}+02$ \\
\hline PRAIRIE ISLAND & 5. 25E+02 & 1.98 & $2.97 E+02$ & $5.64 E+01$ \\
\hline R. E. GINNA & $4.00 E+02$ & 4.60 & 3.76 & $6.35 \mathrm{E}+02$ \\
\hline RANCHO SECO & $4.60 E+02$ & 1.18 & $2.31 E$ & $1.44 E+02$ \\
\hline SALEM $1 \& 2$ & 1.0 & 4.5 & 9.3 & 1. 14E+03 \\
\hline SAN ONOFRE & $7.12 E+02$ & 4.35 & 1.6 & 1. $26 \mathrm{E}+03$ \\
\hline SEQUOYAH & $\mathbf{N} / \mathbf{R}$ & $\mathbf{N}$ & 1.6 & $\mathbf{E}+01$ \\
\hline ST. LUCIE & 3. $12 \mathrm{E}+02$ & 7.4 & 2.5 & $2.96 \mathrm{E}+02$ \\
\hline SURRY & 2.0 & 7.0 & 2.80 & $1.36 \mathrm{E}+03$ \\
\hline THREE MILE ISLAND I & $4.62 \mathrm{E}+02$ & 2.3 & $7.98 E+02$ & $2.34 E+02$ \\
\hline THREE MILE ISLAND 2 & $7.67 E+02$ & 1. 26E+02 & $2.74 E+02$ & $5.11 \mathrm{E}+01$ \\
\hline TMI 2/EPICOR & $\mathbf{N} / \mathbf{R}$ & $\mathbf{N} / \mathbf{R}$ & $1.51 \mathrm{E}+02$ & 3. $50 \mathrm{E}+02$ \\
\hline TROJAN & 5. 14E+02 & $4.59 \mathrm{E}+01$ & $3.75 E+02$ & $1.04 \mathrm{E}+03$ \\
\hline URKEY POINT & $7.24 E+02$ & $1.61 \mathrm{E}+02$ & $1.25 E+03$ & $1.17 \mathrm{E}+02$ \\
\hline YANKEE ROWE & $2.07 E+02$ & $9.57 \mathrm{E}+01$ & $3.08 E+02$ & $6.79 E+01$ \\
\hline ZION & $1.64 \mathrm{E}+03$ & $2.55 E+03$ & 1.53E+03 & $3.44 E+03$ \\
\hline
\end{tabular}


TABLE 13

NET ELECTRICAL ENERGY GENERATION COMPARISON BY YEAR

BOILING WATER REACTORS

$\begin{array}{lr}\text { FACILITY } & \text { CRITICALITY } \\ \text { BIG ROCK POINT } & 09 / 27 / 62 \\ \text { BROWNS FERRY } & 08 / 17 / 73 \\ \text { BROWNS FERRY } & 07 / 20 / 74 \\ \text { BROWNS FERRY } & 08 / 08 / 76 \\ \text { BRUNSWICK } & 10 / 08 / 76 \\ \text { BRUNSWICK } & 03 / 20 / 75 \\ \text { COOPER } & 02 / 21 / 74 \\ \text { DRESDEN 1 } & 10 / 15 / 59 \\ \text { DRESDEN 2-3 } & 01 / 07 / 70 \\ \text { DRESDEN 2-3 } & 01 / 31 / 71 \\ \text { DUANE ARNOLD } & 03 / 23 / 74 \\ \text { EDWIN I. HATCH 1 } & 09 / 12 / 74 \\ \text { EDWIN I. HATCH } 2 & 07 / 04 / 78 \\ \text { HUMBOLDT BAY } & 02 / 16 / 63 \\ \text { JAMES A. FITZPATRICK } 11 / 17 / 74 \\ \text { LACROSSE } & 07 / 11 / 67 \\ \text { MILLSTONE 1 } & 10 / 26 / 70 \\ \text { MONTICELLO } & 12 / 10 / 70 \\ \text { NINE MILE POINT } & 09 / 05 / 69 \\ \text { OYSTER CREEK } & 05 / 03 / 69 \\ \text { PEACH BOTTOM } & 09 / 16 / 73 \\ \text { PEACH BOTTOM } & 08 / 07 / 74 \\ \text { PILGRIM } & 06 / 16 / 72 \\ \text { QUAD-CITIES } & 10 / 18 / 71 \\ \text { QUAD-CITIES } & 04 / 26 / 72 \\ \text { VERMONT YANKEE } & 03 / 24 / 72 \\ \text { FORT ST. VRAIN } & 01 / 31 / 74\end{array}$

\section{MEGAWATT HOURS}

\begin{tabular}{|c|c|c|c|c|c|c|c|}
\hline 1972 & 1973 & 1974 & 1975 & 1976 & 1977 & $\begin{array}{c}1978 \\
4.01 E+05 \\
1.69 E+07\end{array}$ & $\begin{array}{c}1979 \\
1.14 E+05 \\
2.04 E+07\end{array}$ \\
\hline & & & & & & $9.91 \mathrm{E}+06$ & $6.82 E+06$ \\
\hline & & & & & & $4.89 E+06$ & $4.99 E+06$ \\
\hline & & & & & & $7.59 E+05$ & $0.00 \mathrm{E}+00$ \\
\hline & & & & & & $9.53 \mathrm{E}+06$ & $8.42 E+06$ \\
\hline & & & & & & 1. $23 \mathrm{E}+06$ & $2.90 E+06$ \\
\hline & & & & & & 4.77E+06 & 5. $10 \mathrm{E}+06$ \\
\hline & & & & & & $0.00 E+00$ & $0.00 E+00$ \\
\hline & & & & & & $4.20 E+06$ & $2.96 E+06$ \\
\hline & & & & & & $1.74 E+05$ & $2.01 \mathrm{E}+05$ \\
\hline & & & & & & $4.65 E+06$ & $4.22 E+06$ \\
\hline & & & & & & 3.86E+06 & $4.40 E+06$ \\
\hline & & & & & & 4.47E+06 & 3.00E +06 \\
\hline & & & & & & $3.65 E+06$ & $4.56 E+06$ \\
\hline & & & & & & $1.38 E+07$ & $1.47 E+07$ \\
\hline & & & & & & $4.38 E+06$ & $4.84 E+06$ \\
\hline & & & & & & $9.15 E+06$ & $8.76 E+06$ \\
\hline & & & & & & $3.24 E+06$ & $3.45 E+06$ \\
\hline & & & & & & $6.09 \mathrm{E}+05$ & 1.24E+05 \\
\hline
\end{tabular}


TABLE 13

NET ELECTRICAL ENERGY GENERATION COMPARISON BY YEAR

BOILING WATER REACTIORS

INITIAL

FACILITY

BIG ROCK POINT

BROWNS FERRY

BROWNS FERRY

BROWNS FERRY

BRUNSWICK

BRUNSWICK

COOPER

DRESDEN 1

DRESDEN 2-3

DRESDEN 2-3

DUANE ARNOLD

EDWIN I . HATCH

EDWIN 1. HATCH 2

HUMBOLDT BAY

JAMES A. FITZPATR ICK $11 / 17 / 74$

LCROSSE

MILLSTONE

MONTICELLO

NINE MILE POINT

OYSTER CREEK

PEACH BOTTOY

PEACH BOTTOM

PILGRIM

QUAD-CITIES

QUAD-CITIES

VERMONT YANKEE

FORT ST. VRAIN

$09 / 27 / 62$

$08 / 17 / 73$

$07 / 20 / 74$

$08 / 08 / 76$

$0 / 08 / 76$

$03 / 20 / 75$

$02 / 21 / 74$

$10 / 15 / 59$

$01 / 07 / 70$

$01 / 31 / 71$

$03 / 23 / 74$

$09 / 12 / 74$

$07 / 04 / 78$

$07 / 11 / 67$
$10 / 26 / 70$

$12 / 10 / 70$

$09 / 05 / 69$

$05 / 03 / 69$

$09 / 16 / 73$

$08 / 07 / 74$

$06 / 16 / 72$

$10 / 18 / 71$

$04 / 26 / 79$

$03 / 24 / 72$

$01 / 31 / 74$

1980

$+05 \quad 4.70 E+05$

6.06E+06 4.41E+06

5.62E+06 7.47E+06

$6.94 \mathrm{E}+06 \quad 6.26 \mathrm{E}+06$

$3.94 E+06 \quad 2.56 E+06$

1.86E+05 3.28E+06

$3.79 E+06 \quad 3.85 E+06$

$0.00 E+00 \quad 0.00 E+00$

$4.58 \mathrm{E}+06 \quad 3.41 \mathrm{E}+06$

4.33E+06 5.18E+06

$2.80 \mathrm{E}+06 \quad 2.22 \mathrm{E}+06$

4.79E+06 $2.76 E+06$

$3.64 E+06 \quad 4.48 E+06$

$0.00 \mathrm{E}+00 \quad 0.00 \mathrm{E}+00$

4.33E+06 4.78E+06

$2.15 E+06 \quad 2.41 E+05$

$3.40 E+06 \quad 2.52 E+06$

$3.45 E+063.26 E+06$

4.54E+06 3.27E+06

$1.96 \mathrm{E}+06 \quad 2.63 \mathrm{E}+06$

4.34E+06 6.63E+06

7.23E+06 3.13E+06

$3.04 E+063.44 E+06$

$3.44 E+06 \quad 3.73 E+06$

$3.61 \mathrm{E}+06 \quad 3.77 \mathrm{E}+06$

2.98E+06 3.57E+06

6.76E+05 7.55E+05

MEGAWATT HOURS 
TABLE 14

NET ELECTRICAL ENERGY GENERATION COMPARISON BY YEAR

PRESSURIZED WATER REACTORS

INITIAL

FACIIITY

CRITICALITY

ARRANSAS ONE 1

$12 / 05 / 78$

RANSAS ONE 2

$05 / 10 / 76$

BEAVER VALLEYY

CALVERT CLIFFS

$10 / 07 / 74$

CRYSTAL RIVER

$11 / 30 / 76$

$01 / 14 / 77$

DAVIS-BESSE

DONALD C. COOX

$08 / 12 / 77$

$01 / 18 / 73$

DONALD C COO

$07101 / 78$

$08 / 06 / 73$

H. B. ROBINSON

$07 / 24 / 67$

$08 / 02 / 62$

$05 / 22 / 73$

$04 / 06 / 76$

INDIAN POINT $1-2$

JOSEPH $M$. FARLEY 1 08/09/77

JOSEPH $M$. FARLEY 2 05/05/8

KEWAUNEE $\quad 03 / 07 / 74$

MAINE YANKEE $10 / 23 / 72$

MCGUIRE 08/08/8

MILLSTONE $2 \quad 10 / 17 / 75$

NORTH ANNA $04 / 05 / 78$

NORTH ANNA $\quad 06 / 12 / 80$

OCONEE

$04 / 19 / 73$

OCONEE

$11 / 11 / 73$

OCONEE

$09 / 05 / 74$

$05 / 24 / 71$

POINT BEACH

POINT BEACH

$05 / 30 / 72$

$12 / 01 / 73$

PRAIRIE ISLAND $12 / 17 / 74$

R. E. CINNA

$\begin{array}{ll}\text { RANCHO SECO } & 09 / 16 / 74 \\ \text { SALEM I } & 12 / 11 / 76\end{array}$

SALEM 1

$08 / 02 / 80$

$06 / 14 / 67$

SAN ONOFRE

$07 / 05 / 80$

SEQUOYAH

$\begin{array}{ll}\text { ST. LUCIE } & 04 / 22 / 76 \\ \text { SURRY } & 07 / 01 / 72\end{array}$

SURRY

$03 / 07 / 73$

THREE MILE ISLAND I 06/05/74

THREE MILE ISLAND $203 / 28 / 78$

TMI 2/EPICOR $03 / 28 / 78$

TROJ AN

$12 / 15 / 75$

TURKEY POINT

$10 / 20 / 72$

$06 / 11 / 73$

$08 / 19 / 60$

$06 / 10 / 73$

$12 / 24 / 73$
MEGAWATT HOURS

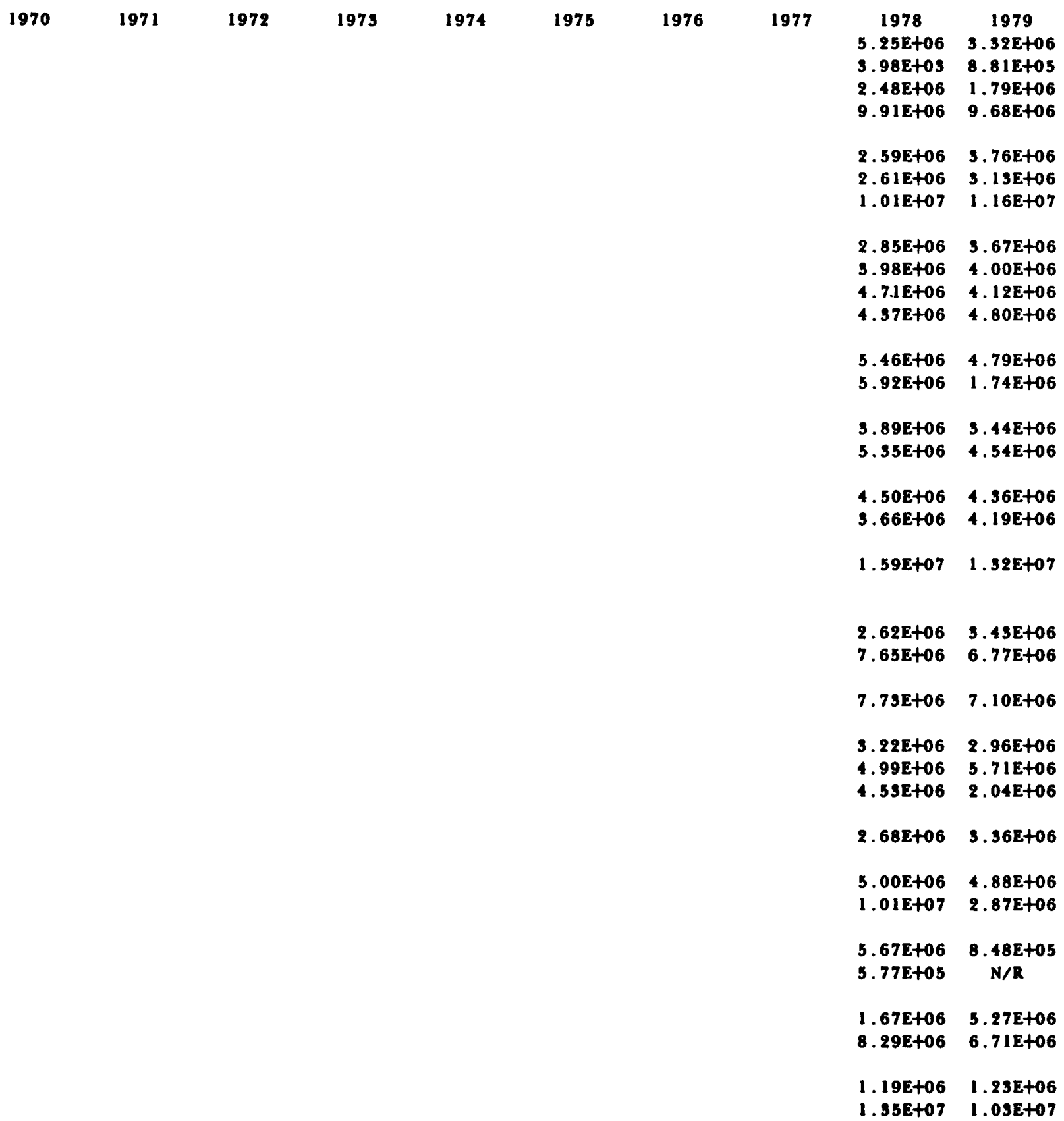

$1.35 E+07 \quad 1.035+07$ 
TABLE 14

NET ELECTR ICAL ENERGY GENERATION COMPARISON BY YEAR

PRESSURIZED WATER REACTORS

INITIAL

\begin{tabular}{|c|c|c|c|}
\hline FACILITY & CRITICALITY & 1980 & 1981 \\
\hline ARKANSAS ONE 1 & $08 / 06 / 74$ & 3.78E+06 & 4. $90 \mathrm{E}+06$ \\
\hline ARKANSAS ONE 2 & $12 / 05 / 78$ & $3.65 E+06$ & $4.32 E+06$ \\
\hline BEAVER VALLEY & $05 / 10 / 76$ & $3.01 E+05$ & $4.66 E+06$ \\
\hline CALVERT CLIFFS & $10 / 07 / 74$ & $4.53 E+06$ & $6.11 E+06$ \\
\hline CALVERT CLIFFS & $11 / 30 / 76$ & $6.41 E+06$ & $5.42 E+06$ \\
\hline CRYSTAL RIVER & $01 / 14 / 77$ & 3.35E+06 & $4.01 E+06$ \\
\hline DAVIS-BESSE & $08 / 12 / 77$ & $2.09 E+06$ & $4.36 E+06$ \\
\hline DONALD C. COOK & $01 / 18 / 75$ & $6.46 E+06$ & $6.78 E+06$ \\
\hline DONALD C. COOK & $07 / 01 / 78$ & $6.70 E+06$ & $6.38 \mathrm{E}+06$ \\
\hline FORT CALHOUN & $08 / 06 / 73$ & $2.01 E+06$ & $2.15 E+06$ \\
\hline H. B. ROB I NSON & $09 / 20 / 70$ & 3.21E+06 & 3. $50 \mathrm{E}+06$ \\
\hline HADDAM NECK & $07 / 24 / 67$ & $3.56 \mathrm{E}+06$ & $a+06$ \\
\hline INDIAN POINT 1-2 & $08 / 02 / 62$ & $0.00 E+00$ & +00 \\
\hline INDIAN POINT 1-2 & $05 / 22 / 73$ & $4.26 E+06$ & $2+06$ \\
\hline INDIAN POINT 3 & 04/06/76 & 3.07E+06 & $3.03 E+06$ \\
\hline JOSEPH M. FARLEY I & $08 / 09 / 77$ & $4.60 \mathrm{E}+06$ & $2.62 E+06$ \\
\hline JOSEPH M. FARLEY 2 & $05 / 05 / 81$ & & $2.92 E+06$ \\
\hline KEWAUNEE & $03 / 07 / 74$ & 3.63E+06 & 3. $77 E+06$ \\
\hline MAINE YANKEE & $10 / 23 / 72$ & $4.40 E+06$ & $5.21 E+06$ \\
\hline MCGUIRE & $08 / 08 / 81$ & & $1.91 E+04$ \\
\hline MILLSTONE 2 & $10 / 17 / 75$ & $4.88 E+06$ & $6.09 \mathrm{E}+06$ \\
\hline NORTH ANNA & $04 / 05 / 78$ & $5.63 E+06$ & $4.64 E+06$ \\
\hline NORTH ANNA & $06 / 12 / 80$ & $3.50 E+05$ & $5.65 E+06$ \\
\hline OCONEE & $04 / 19 / 73$ & $5.12 E+06$ & $3.00 E+06$ \\
\hline OCONEE & $11 / 11 / 73$ & 3.. 88E+06 & $5.19 E+06$ \\
\hline OCONEE & $5 / 74$ & $5.22 E+06$ & $5.64 E+06$ \\
\hline PALI SADES & $4 / 71$ & $2.38 E+06$ & $3.46 \mathrm{E}+06$ \\
\hline POINT BEACH & $2 / 70$ & $2.48 E+06$ & 2.61 \\
\hline POINT BEACH & $1 / 72$ & 3.59E+06 & 3.7 \\
\hline PRAIRIE ISLAND & $1 / 73$ & $3.11 E+06$ & 3.8 \\
\hline PRAIRIE ISLAND & $7 / 74$ & 3.471 & 3.0 \\
\hline R. E. GINNA & $8 / 69$ & $3.09 E+06$ & $E+06$ \\
\hline RANCHO SECO & $09 / 16 / 74$ & 4.42E+06 & $2.63 \mathrm{E}+06$ \\
\hline SALEM 1 & $12 / 11 / 76$ & $5.68 E+06$ & $6.19 E+06$ \\
\hline SALEM 2 & $08 / 02 / 80$ & $0.00 E+00$ & $1.63 \mathrm{E}+06$ \\
\hline SAN ONOFRE & $06 / 14 / 67$ & $8.17 E+05$ & $7.79 E+05$ \\
\hline SEQUOYAH & $07 / 05 / 80$ & $5.18 E+05$ & 2.53E+06 \\
\hline ST. LUCIE & $04 / 22 / 76$ & $5.20 E+06$ & $4.95 E+06$ \\
\hline SURRY & $07 / 01 / 72$ & $2.47 E+06$ & $2.38 E+06$ \\
\hline SURRY & $03 / 07 / 73$ & $2.24 E+06$ & 5.15E+06 \\
\hline THREE MILE ISLAND & $06 / 05 / 74$ & $0.00 E+00$ & $0.00 \mathrm{E}+00$ \\
\hline THREE MILE ISLAND 2 & $03 / 28 / 78$ & $0.00 E+00$ & $0.00 E+00$ \\
\hline TMI 2/EPICOR & $03 / 28 / 78$ & $0.00 E+00$ & $0.00 E+00$ \\
\hline TROJAN & $12 / 15 / 75$ & 6.07E+06 & $6.42 E+06$ \\
\hline TURKEY POINT & $10 / 20 / 72$ & $4.39 E+06$ & $9.12 E+05$ \\
\hline TURKEY POINT & $06 / 11 / 73$ & $3.85 E+06$ & $4.50 \mathrm{E}+06$ \\
\hline ZANKEE ROWI & $08 / 19 / 60$ & $2.92 E+05$ & $8.85 E+05$ \\
\hline 1 & $06 / 19 / 73$ & $6.51 E+06$ & $6.19 E+06$ \\
\hline & $12 / 24 / 73$ & $5.28 E+06$ & $5.26 E+06$ \\
\hline
\end{tabular}
3.78E+06

\section{MEGAWATT HOURS}

6

.

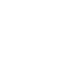

6


TABLE 15

THERMAL ENERGY GENERATION COMPARISON BY YEAR

BOILING WATER RFACTORS

$\begin{array}{lr}\text { FACILITY } & \text { CRITICALITY } \\ \text { BIG ROCK POINT } & 09 / 27 / 62 \\ \text { BROWNS FERRY } & 08 / 17 / 79 \\ \text { BROWNS FERRY } & 07 / 20 / 74 \\ \text { BROWNS FERRY } & 08 / 08 / 76 \\ \text { BRUNSWICK } & 10 / 08 / 76 \\ \text { BRUNSWICK } & 03 / 20 / 75 \\ \text { COOPER } & 02 / 21 / 74 \\ \text { DRESDEN 1 } & 10 / 15 / 59 \\ \text { DRESDEN 2-3 } & 01 / 07 / 70 \\ \text { DRESDEN 2-3 } & 01 / 31 / 71 \\ \text { DUANE ARNOLD } & 03 / 23 / 74 \\ \text { EDWIN I. HATCH 1 } & 09 / 12 / 74 \\ \text { EDWIN I HATCH } 2 & 07 / 04 / 78 \\ \text { HUMBOLDT. BAY } & 02 / 16 / 63 \\ \text { JAMES A. FITZPATRICK } 11 / 17 / 74 \\ \text { LACROSSE } & 07 / 11 / 67 \\ \text { MILLSTONE 1 } & 10 / 26 / 70 \\ \text { MONTICELLO } & 12 / 10 / 70 \\ \text { NINE MILE POINT } & 09 / 05 / 69 \\ \text { OYSTER CREEK } & 05 / 03 / 69 \\ \text { PEACH BOTTOM } & 09 / 16 / 73 \\ \text { PEACH BOTTOM } & 08 / 07 / 74 \\ \text { PILGRIM } & 06 / 16 / 72 \\ \text { QUAD-CITIES } & 10 / 18 / 71 \\ \text { QUAD-CITIES } & 04 / 26 / 72 \\ \text { VERMONT YANKEE } & 03 / 24 / 72 \\ \text { FORT ST. VRAIN* } & 01 / 31 / 74\end{array}$

\section{MEGAWATT HOURS}

\begin{tabular}{|c|c|c|c|c|c|c|c|c|c|}
\hline 1970 & 1971 & 1972 & 1973 & 1974 & 1975 & 1976 & 1977 & 1978 & 1979 \\
\hline \multirow[t]{4}{*}{ 1. $18 E+06$} & $1.21 \mathrm{E}+06$ & $1.20 \mathrm{E}+06$ & $1.41 \mathrm{E}+06$ & $1.13 \mathrm{E}+06$ & $9.80 \mathrm{E}+05$ & $8.30 \mathrm{E}+05$ & $1.23 E+06$ & $1.37 \mathrm{E}+06$ & 3. $96 \mathrm{E}+05$ \\
\hline & & & 1. $37 E+06$ & $1.64 E+07$ & $8.75 \mathrm{E}+06$ & $1.34 \mathrm{E}+07$ & $5.37 E+07$ & $5.32 \mathrm{E}+07$ & $6.31 E+07$ \\
\hline & & & & & $4.72 E+06$ & $7.81 E+06$ & 1.59E+07 & 3.07E+07 & $2.12 \mathrm{E}+07$ \\
\hline & & & & $6.90 E+06$ & 1. $24 E+07$ & $1.19 E+07$ & $1.45 E+07$ & $1.54 \mathrm{E}+07$ & $1.58 \mathrm{E}+07$ \\
\hline $4.77 E+06$ & $2.38 E+06$ & $3.76 \mathrm{E}+06$ & $2.43 E+06$ & 1. $36 E+06$ & $2.56 E+06$ & $3.42 E+06$ & $2.21 E+06$ & $2.73 E+06$ & $0.00 E+00$ \\
\hline \multirow[t]{3}{*}{ 4. $11 \mathrm{E}+06$} & 1. $23 \mathrm{E}+07$ & $2.52 \mathrm{E}+07$ & $2.83 \mathrm{E}+07$ & 2. $18 E+07$ & $1.70 E+07$ & $2.74 E+07$ & $2.89 E+07$ & 3. $13 E+07$ & $2.77 E+07$ \\
\hline & & & & & $7.42 \mathrm{E}+06$ & $8.02 E+06$ & $9.32 \mathrm{E}+06$ & $3.96 \mathrm{E}+06$ & $9.07 \mathrm{E}+06$ \\
\hline & & & & & $9.75 \mathrm{E}+06$ & $1.38 E+07$ & $1.22 E+07$ & 3.37E+07 & $1.62 \mathrm{E}+07$ \\
\hline \multirow[t]{2}{*}{ 1. $40 \mathrm{E}+06$} & 1. $14 \mathrm{E}+06$ & $1.25 E+06$ & $1.47 \mathrm{E}+06$ & $1.27 E+06$ & $1.32 \mathrm{E}+06$ & $6.80 \mathrm{E}+05$ & $0.00 \mathrm{E}+00$ & $0.00 E+00$ & $0.00 \mathrm{E}+00$ \\
\hline & & & & & $6.81 E+06$ & $1.26 E+07$ & $1.18 E+07$ & $1.30 \mathrm{E}+07$ & $8.97 E+06$ \\
\hline $4.50 E+05$ & $7.10 E+05$ & $8.20 E+05$ & $6.90 E+05$ & $1.08 E+06$ & $9.20 \mathrm{E}+05$ & $6.10 \mathrm{E}+05$ & 3. 43E +05 & $6.54 E+05$ & $7.48 \mathrm{E}+05$ \\
\hline \multirow[t]{2}{*}{$2.60 E+05$} & $1.11 \mathrm{E}+07$ & $9.69 \mathrm{E}+06$ & $5.96 \mathrm{E}+06$ & 1. $12 E+07$ & $1.21 E+07$ & $1.16 E+07$ & $1.48 E+07$ & $1.43 E+07$ & $1.30 E+07$ \\
\hline & $4.37 E+06$ & $1.10 \mathrm{E}+07$ & $9.90 E+06$ & $8.28 E+06$ & $8.88 E+06$ & $1.23 E+07$ & 1. 10E+07 & 1. $18 E+07$ & $1.35 E+07$ \\
\hline $5.94 E+06$ & $9.94 \mathrm{E}+06$ & $1.00 E+07$ & $1.10 E+07$ & 1. $05 \mathrm{E}+07$ & $9.68 E+06$ & $1.31 E+07$ & $9.15 E+06$ & 1. $39 E+07$ & $9.67 E+06$ \\
\hline \multirow{5}{*}{$1.06 \mathrm{E}+07$} & 1. 17E+07 & $1.30 \mathrm{E}+07$ & $1.09 \mathrm{E}+07$ & 1. $13 \mathrm{E}+07$ & $9.81 \mathrm{E}+06$ & $1.18 E+07$ & $9.82 E+06$ & 1. 10E+07 & 1. $38 E+07$ \\
\hline & & & & 1. $23 E+07$ & $3.34 E+07$ & 3. $72 \mathrm{E}+07$ & $2.86 E+07$ & $4.39 E+07$ & $4.58 \mathrm{E}+07$ \\
\hline & & $2.65 \mathrm{E}+06$ & $1.25 \mathrm{E}+07$ & $6.00 E+06$ & $8.10 E+06$ & $7.60 \mathrm{E}+06$ & $8.26 E+06$ & 1.33E+07 & $1.47 E+07$ \\
\hline & & $1.25 E+07$ & 3. $17 \mathrm{E}+07$ & $2.61 \mathrm{E}+07$ & $2.31 \mathrm{E}+07$ & $2.59 \mathrm{E}+07$ & $2.68 E+07$ & 3. $14 \mathrm{E}+07$ & 3. $00 \mathrm{E}+07$ \\
\hline & & $1.48 \mathrm{E}+06$ & $6.08 E+06$ & $8.20 E+06$ & $1.13 \mathrm{E}+07$ & $1.02 E+07$ & $1.11 E+07$ & $\begin{array}{l}1.00 E+07 \\
2.04 E+06\end{array}$ & $\begin{array}{l}1.08 E+07 \\
4.78 E+05\end{array}$ \\
\hline
\end{tabular}


TABLE 15

THERMAL ENERGY GENERATION COMPARISON BY YEAR

BOILING WATER REACTORS

INITIAL

FACILITY

BIC ROCK POINT

BROWNS FERRY $08 / 17 / 73$

BROWNS FERRY $07 / 20 / 74$

BROWNS FERRY $08 / 08 / 76$

CORUNS

DRESDEN

DRESDEN 2-3

DRESDEN 2-3

DUANE ARNOLD

EDWIN I. HATCH 1

EDWIN I . HATCH 2

HUMBOLDT BAY

LACROSSE

MILLSTONE 1

MONTICELLO

NINE MILE POINT

OYSTER CREEK

PEACH BOTTOM

PEACH BOTTOM

PILGRIM

QUAD-CITIES

QUAD-CITIES

VERMONT YANKEE

FORT ST. VRAIN
$10 / 08 / 76$

$03 / 20 / 75$

$02 / 21 / 74$

$10 / 15 / 59$

$01 / 07 / 70$

$01 / 31 / 71$

$03 / 23 / 74$

$09 / 12 / 74$

$07 / 04 / 78$

$02 / 16 / 63$

$07 / 11 / 67$

$10 / 26 / 70$

$12 / 10 / 70$

$09 / 05 / 69$

$05 / 03 / 69$

$09 / 16 / 73$

$08 / 07 / 74$

$06 / 16 / 72$

$10 / 18 / 71$

$04 / 26 / 72$

$01 / 31 / 74$
MEGAWATT HOURS

$\begin{array}{cc}1980 & 1981 \\ 1.40 E+06 & 1.63 E+06 \\ 1.92 E+07 & 1.36 E+07 \\ 1.74 E+07 & 2.31 E+07 \\ 2.13 E+07 & 1.95 E+07 \\ 1.23 E+07 & 8.29 E+06 \\ 5.38 E+06 & 1.04 E+07 \\ 1.36 E+07 & 1.39 E+07 \\ 0.00 E+00 & 0.00 E+00 \\ 1.57 E+07 & 1.13 E+07 \\ 1.42 E+07 & 1.71 E+07 \\ 8.87 E+06 & 7.05 E+06 \\ 1.54 E+07 & 8.97 E+06 \\ 1.16 E+07 & 1.47 E+07 \\ 0.00 E+00 & 0.00 E+00 \\ 1.30 E+07 & 1.42 E+07 \\ 8.00 E+05 & 9.11 E+05 \\ 1.04 E+07 & 8.60 E+06 \\ 1.07 E+07 & 1.01 E+07 \\ 1.41 E+07 & 1.01 E+07 \\ 6.27 E+06 & 8.44 E+06 \\ 1.37 E+07 & 2.08 E+07 \\ 2.26 E+07 & 9.85 E+06 \\ 9.20 E+06 & 1.05 E+07 \\ 1.17 E+07 & 1.88 E+07 \\ 1.22 E+07 & 1.27 E+07 \\ 9.38 E+06 & 1.13 E+07 \\ 2.23 E+06 & 2.23 E+06\end{array}$

$2.23 E+06 \quad 2.23 E+06$ 
TABLE 16

THERMAL ENERGY GENERATION COMPARISON BY YEAR

PRESSURIZED WATER REACTORS

INITIAL

\section{FACILITY}

ARKANSAS ONE 1

CRITICAI

BEAVER VALLEY

CALVERT CLIFFS $\quad 10 / 07 / 74$

CALVERT CLIFFS $\quad 11 / 30 / 76$

CRYSTAL RIVER $01 / 14 / 77$

DAVIS-BESSE $08 / 12 / 77$

DONALD C. COOK $01 / 18 / 75$

DONALD C. COOK $07 / 01 / 78$

FORT CALHOUN $08 / 06 / 73$

H. B. ROBINSON $09 / 20 / 70$

HADDAM NECK $07 / 24 / 67$

INDIAN POINT 1-2 08/02/62

INDIAN POINT 1-2 $05 / 22 / 73$

INDIAN POINT $3 \quad 04 / 06 / 76$

JOSEPH $M$. FARLEY $1 \quad 08 / 09 / 77$

JOSEPH $M$. FARLEY $205 / 05 / 81$

KEWAUNEE $\quad 03 / 07 / 74$

MAINE YANKEE $10 / 23 / 72$

MCGUIRE $08 / 08 / 8$

MILLSTONE $2 \quad 10 / 17 / 75$

NORTH ANNA $\quad 04 / 05 / 78$

NORTH ANNA $06 / 12 / 80$

OCONEE $\quad 04 / 19 / 79$

OCONEE $\quad 11 / 11 / 79$

OCONEE $\quad 09 / 05 / 74$

PALISADES $\quad 05 / 24 / 71$

POINT BEACH $11 / 02 / 70$

POINT BEACH 05/30/72

PRAIRIE ISLAND $12 / 01 / 79$

PRAIRIE ISLAND $12 / 17 / 74$

R. E. GINNA $11 / 08 / 69$

RANCHO SECO $09 / 16 / 74$

SALEM $1 \quad 12 / 11 / 76$

SALEM 2

SAN ONOFRE $\quad 06 / 14 / 67$

SEQUOYAH $07 / 05 / 80$

ST. LUCIE $04 / 22 / 76$

SURRY 07/01/72

SURRY $\quad 03 / 07 / 73$

THREE MILE ISLAND 1 06/05/74

THREE MILE ISLAND $203 / 28 / 78$

TMI 2/EPICOR $03 / 28 / 78$

TROJAN

TURKEY POINT $10 / 20 / 72$

TURKEY POINT $06 / 11 / 73$

YANKEE ROWE $08 / 19 / 60$

ZION $1 \quad 06 / 19 / 73$

ZION $212 / 24 / 79$

MEGAWATT HOURS

\begin{tabular}{|c|c|c|c|c|c|c|c|c|c|}
\hline 1970 & 1971 & 1972 & 1973 & 1974 & 1975 & 1976 & 1977 & 1978 & 1979 \\
\hline & & & & $1.99 E+06$ & $1.54 \mathrm{E}+07$ & $1.21 \mathrm{E}+07$ & $1.64 \mathrm{E}+07$ & $1.64 E+07$ & $1.05 E+07$ \\
\hline & & & & & & & & $4.45 E+04$ & $3.45 E+06$ \\
\hline & & & & & & 1.97E+06 & $1.01 E+07$ & $8.80 E+06$ & $6.11 E+06$ \\
\hline & & & & & $1.40 E+07$ & $1.98 E+07$ & $2.97 E+07$ & 3. $14 E+07$ & 3. $15 E+07$ \\
\hline & & & & & & & $1.26 \mathrm{E}+07$ & $7.97 E+06$ & $1.17 E+07$ \\
\hline & & & & & & & $1.66 \mathrm{E}+06$ & 8. $52 \mathrm{E}+06$ & $1.00 E+07$ \\
\hline & & & & & 1. $46 \mathrm{E}+07$ & $2.15 \mathrm{E}+07$ & $1.55 \mathrm{E}+07$ & $3.29 E+07$ & 3. $68 \mathrm{E}+07$ \\
\hline & & & $2.03 E+06$ & $7.58 E+06$ & $6.71 E+06$ & $7.15 E+06$ & $9.40 \mathrm{E}+06$ & $8.98 E+06$ & $1.16 \mathrm{E}+07$ \\
\hline $6.00 \mathrm{E}+04$ & $7.85 E+06$ & 1. $55 E+07$ & $1.25 E+07$ & $1.56 E+07$ & $1.36 E+07$ & 1. $59 E+07$ & $1.43 E+07$ & 1. $33 E+07$ & $1.30 E+07$ \\
\hline $1.14 E+07$ & $1.34 \mathrm{E}+07$ & $1.38 E+07$ & $7.73 E+06$ & $1.42 E+07$ & $1.34 \mathrm{E}+07$ & 1. $30 \mathrm{E}+07$ & $1.31 E+07$ & $1.51 \mathrm{E}+07$ & 1. $32 \mathrm{E}+07$ \\
\hline & & & $1.47 \mathrm{E}+06$ & $1.15 \mathrm{E}+07$ & 1. $64 \mathrm{E}+07$ & $7.60 \mathrm{E}+06$ & $3.50 E+07$ & $1.48 E+07$ & $1.61 \mathrm{E}+07$ \\
\hline & & & & & & & & $1.70 \mathrm{E}+07$ & $1.57 \mathrm{E}+07$ \\
\hline & & & & & & & & $1.95 \mathrm{E}+07$ & 5. $77 \mathrm{E}+06$ \\
\hline & & & & $5.03 E+06$ & $1.08 \mathrm{E}+07$ & $1.08 E+07$ & $1.11 \mathrm{E}+07$ & $1.24 \mathrm{E}+07$ & $1.09 \mathrm{E}+07$ \\
\hline & & $1.44 E+06$ & $1.08 \mathrm{E}+07$ & $1.14 E+07$ & $1.47 \mathrm{E}+07$ & $1.94 \mathrm{E}+07$ & $1.65 \mathrm{E}+07$ & $1.69 E+07$ & $1.41 \mathrm{E}+07$ \\
\hline & & & & & $6.40 E+05$ & 1. $.52 E+07$ & 1. $42 E+07$ & $1.44 E+07$ & 1. $38 E+07$ \\
\hline & & & & & & & & 1. $22 E+07$ & $1.41 \mathrm{E}+07$ \\
\hline & & & $6.62 \mathrm{E}+06$ & $1.70 \mathrm{E}+07$ & $4.68 E+07$ & $3.97 \mathrm{E}+07$ & $4.00 \mathrm{E}+07$ & $4.84 E+07$ & $4.37 \mathrm{E}+07$ \\
\hline & & $5.91 \mathrm{E}+06$ & $7.80 \mathrm{E}+06$ & 4.00E+05 & 8.91E+06 & $9.66 E+06$ & $1.73 E+07$ & $9.44 E+06$ & 1. 20E +07 \\
\hline $6.30 \mathrm{E}+05$ & $1.00 \mathrm{E}+07$ & $9.96 \mathrm{E}+06$ & $1.84 \mathrm{E}+07$ & $2.04 \mathrm{E}+07$ & $2.09 E+07$ & $2.18 E+07$ & $2.23 \mathrm{E}+07$ & $2.33 E+07$ & $2.08 E+07$ \\
\hline & & & 1. $28 \mathrm{E}+05$ & $5.26 \mathrm{E}+06$ & $2.25 E+07$ & $2.06 E+07$ & $2.46 E+07$ & $2.52 E+07$ & 1. $12 \mathrm{E}+08$ \\
\hline $6.84 \mathrm{E}+06$ & $8.50 E+06$ & $7.71 \mathrm{E}+06$ & $1.08 E+07$ & $6.71 \mathrm{E}+06$ & $9.71 \mathrm{E}+06$ & $6.98 \mathrm{E}+06$ & $1.11 \mathrm{E}+07$ & $1.05 \mathrm{E}+07$ & $9.35 \mathrm{E}+06$ \\
\hline & & & & & $4.11 E+06$ & $6.91 \mathrm{E}+06$ & $1.81 E+07$ & 1.59E+07 & 1. $79 E+07$ \\
\hline & & & & & & $5.00 E+04$ & $6.70 E+06$ & $1.43 \mathrm{E}+07$ & $6.60 E+06$ \\
\hline 9. $19 E+06$ & $9.60 \mathrm{E}+06$ & $8.53 E+06$ & $7.09 E+06$ & $9.73 E+06$ & 1. $.00 E+07$ & $7.75 \mathrm{E}+06$ & $7.29 \mathrm{E}+06$ & $8.54 \mathrm{E}+06$ & $1.05 \mathrm{E}+07$ \\
\hline & & & & & & 3. $50 \mathrm{E}+05$ & $1.75 E+07$ & $1.64 \mathrm{E}+07$ & $1.60 E+07$ \\
\hline & & 1. $29 \mathrm{E}+06$ & $2.26 \mathrm{E}+07$ & $1.92 \mathrm{E}+07$ & $2.90 \mathrm{E}+07$ & $2.51 \mathrm{E}+07$ & 3. $10 E+07$ & 3. $27 E+07$ & $9.32 \mathrm{E}+06$ \\
\hline & & & & $6.20 E+06$ & 1. $76 E+07$ & $1.39 E+07$ & $1.76 E+07$ & $1.83 \mathrm{E}+07$ & $2.83 E+06$ \\
\hline & & & & & & & & 3. $16 E+06$ & $\mathbf{N} / \mathbf{R}$ \\
\hline & & & & & & $7.54 \mathrm{E}+06$ & $2.12 E+07$ & $5.63 \mathrm{E}+06$ & $1.70 \mathrm{E}+07$ \\
\hline & & $2.90 \mathrm{E}+05$ & $1.53 \mathrm{E}+07$ & $2.55 E+07$ & $2.78 E+07$ & $2.68 E+07$ & $2.70 \mathrm{E}+07$ & $2.81 E+07$ & $2.28 E+07$ \\
\hline $4.18 E+06$ & 5.02E+06 & $2.40 E+06$ & $3.57 \mathrm{E}+06$ & 3.07E+06 & $4.02 E+06$ & $4.25 E+06$ & 3. $52 \mathrm{E}+06$ & $4.16 E+06$ & 4. $17 \mathrm{E}+06$ \\
\hline & & & $2.73 E+06$ & $1.69 \mathrm{E}+07$ & 3. 28E+07 & 3.11E+07 & $3.66 E+07$ & $4.30 E+07$ & 3.31E+07 \\
\hline
\end{tabular}


TABLE 16

THERMAL ENERGY GENERATION COMPARISON BY YEAR

PRESSURIZED WATER REACTORS

INITIAL

FACILITY

ARKANSAS ONE

ARKANSAS ONE 2

BEAVER VALLEY

CALVERT GLIFFS

CALVERT CLIFFS

CRYSTAL RIVER

DAVIS-BESSE

DONALD C. COOK

FORT GALHOUN

H. B. ROBINSON

HADDAM NECK

INDIAN POINT 1-2

INDIAN POINT 1-2

INDIAN POINT 3

JOSEPH M. FARLEY 1

JOSEPH M. FARLEY 2

KEWAUNEE

MAINE YANKEE

MCGUIRE

MILLSTONE 2

NORTH ANNA

NORTH ANNA

OCONEE

OCONEE

OCONEE

PALISADES

POINT BEACH

POINT BEACH

PRAIRIE ISLAND

PRAIRIE ISLAND

R. E. GINNA

RANCHO SECO

SALEM 1

SALEM 2

SAN ONOFRE

SEQUOYAH

ST. LUC1E

SURRY

SURRY

THREE MILE ISLAND 1

THREE MILE ISLAND 2

TMI 2/EPICOR

TROJAN

TURKEY POINT

TURKEY POINT

YANKEE ROWE

TON 1

$\begin{array}{ccc}\text { CRITICALITY } & 1980 & 1981 \\ 08 / 06 / 74 & 1.29 E+07 & 1.54 E+07 \\ 12 / 05 / 78 & 1.18 E+07 & 1.39 E+07 \\ 05 / 10 / 76 & 1.13 E+06 & 1.55 E+07 \\ 10 / 07 / 74 & 1.52 E+07 & 1.96 E+07 \\ 11 / 30 / 76 & 2.05 E+07 & 1.71 E+07 \\ 01 / 14 / 77 & 1.04 E+07 & 1.27 E+07 \\ 08 / 12 / 77 & 6.71 E+06 & 1.40 E+07 \\ 01 / 18 / 75 & 2.02 E+07 & 2.11 E+07 \\ 07 / 01 / 78 & 2.14 E+07 & 2.04 E+07 \\ 08 / 06 / 73 & 6.48 E+06 & 6.98 E+06 \\ 09 / 20 / 70 & 1.07 E+07 & 1.19 E+07 \\ 07 / 24 / 67 & 1.14 E+07 & 1.31 E+07 \\ 08 / 02 / 62 & 0.00 E+00 & 0.00 E+00 \\ 05 / 22 / 73 & 1.50 E+07 & 1.05 E+07 \\ 04 / 06 / 76 & 1.15 E+07 & 1.14 E+07 \\ 08 / 09 / 77 & 1.54 E+07 & 8.96 E+06 \\ 05 / 05 / 81 & & 9.47 E+06 \\ 03 / 07 / 74 & 1.15 E+07 & 1.21 E+07 \\ 10 / 23 / 72 & 1.41 E+07 & 1.67 E+07 \\ 08 / 08 / 81 & & 8.50 E+04 \\ 10 / 17 / 75 & 1.55 E+07 & 1.92 E+07 \\ 04 / 05 / 78 & 1.89 E+07 & 1.51 E+07 \\ 06 / 12 / 80 & 1.12 E+06 & 1.77 E+07 \\ 04 / 19 / 73 & 1.52 E+07 & 9.00 E+06 \\ 11 / 11 / 73 & 1.20 E+07 & 1.59 E+07 \\ 09 / 05 / 74 & 1.59 E+07 & 1.72 E+07 \\ 05 / 24 / 71 & 8.19 E+06 & 1.17 E+07 \\ 11 / 02 / 70 & 8.09 E+06 & 8.51 E+06 \\ 05 / 30 / 72 & 1.11 E+07 & 1.15 E+07 \\ 12 / 01 / 73 & 1.06 E+07 & 1.25 E+07 \\ 12 / 17 / 74 & 1.14 E+07 & 1.01 E+07 \\ 11 / 08 / 69 & 9.93 E+06 & 1.07 E+07 \\ 09 / 16 / 74 & 1.39 E+07 & 8.92 E+06 \\ 12 / 11 / 76 & 1.84 E+07 & 2.02 E+07 \\ 08 / 02 / 80 & 0.00 E+00 & 5.11 E+06 \\ 06 / 14 / 67 & 2.55 E+06 & 2.59 E+06 \\ 07 / 05 / 80 & 1.67 E+06 & 8.06 E+06 \\ 04 / 22 / 76 & 1.70 E+07 & 1.61 E+07 \\ 07 / 01 / 72 & 8.67 E+06 & 7.94 E+06 \\ 03 / 07 / 73 & 7.26 E+06 & 1.68 E+07 \\ 06 / 05 / 74 & 0.00 E+00 & 0.00 E+00 \\ 03 / 28 / 78 & 0.00 E+00 & 0.00 E+00 \\ 03 / 28 / 78 & 0.00 E+00 & 0.00 E+00 \\ 12 / 15 / 75 & 1.97 E+07 & 2.10 E+07 \\ 10 / 20 / 72 & 1.47 E+07 & 3.03 E+06 \\ 06 / 11 / 73 & 1.32 E+07 & 1.48 E+07 \\ 08 / 19 / 60 & 1.13 E+06 & 3.63 E+06 \\ 06 / 19 / 73 & 2.22 E+07 & 1.98 E+07 \\ 12 / 24 / 73 & 1.75 E+07 & 1.77 E+07\end{array}$

\section{MEGAWATT HOURS}


APPENDIX A

INDIVIDUAL PLANT SUMMARIES

$A-1$ 
EFFLUENT AND WASTE DISPOSAL ANNUAL REPORT FOR YEAR 1981 AIRBORNE AND LIQUID EFFLUENTS

UNIT NUMBER: I

TYPE : PWR

DOCKET NO. : 50-313

COOLING WATER SOURCE:DARDANELLE RESERVOIR
LICENSEE : ARKANSAS POWER \&LIGHT

LICENSED POWER (WWT) : 2568.00 INITIAL CRITICALITY:08/06/74

\begin{tabular}{|c|c|}
\hline \\
\hline $\begin{array}{l}\text { AIRBORNE EFFLUENTS } \\
\text { NUCLIDES RELEASED }\end{array}$ & ACTIVITY (CI) \\
\hline$K-40$ & 1. 36E-05 \\
\hline AR-41 & $0.00 E+00$ \\
\hline MN-54 & $7.14 E-07$ \\
\hline $\mathrm{CO}-58$ & $1.45 \mathrm{E}-05$ \\
\hline C0-60 & $6.36 \mathrm{E}-06$ \\
\hline KR-85 & $5.40 E+01$ \\
\hline KR-85M & $1.41 \mathrm{E}-02$ \\
\hline KR-87 & $0.00 E+00$ \\
\hline KR-88 & $1.41 \mathrm{E}-03$ \\
\hline SR-89 & $7.16 E-07$ \\
\hline SR-90 & $6.95 E-07$ \\
\hline NB-95 & 9. $40 E-07$ \\
\hline$I-191$ & 5.52E-03 \\
\hline$X E-131 M$ & $1.45 E+02$ \\
\hline $1-133$ & $7.10 \mathrm{E}-05$ \\
\hline $\mathrm{XE}-133$ & $3.43 E+03$ \\
\hline$X E-133 M$ & 5. $70 E+00$ \\
\hline Cs-134 & 3. $12 E-06$ \\
\hline$X E-135$ & $9.91 E+01$ \\
\hline CS-137 & $1.42 E-05$ \\
\hline$B A-140$ & $2.02 E-07$ \\
\hline TH-228 & 3.02E-06 \\
\hline LIQUID EFFLUENTS & \\
\hline NUCLIDES RELEASED & ACTIVITY (CI) \\
\hline BE-7 & $6.68 E-03$ \\
\hline NA-24 & $6.51 E-04$ \\
\hline CR-51 & $2.18 \mathrm{E}-01$ \\
\hline$M N-54$ & $4.98 \mathrm{E}-02$ \\
\hline$M N-56$ & $0.00 E+00$ \\
\hline $\mathrm{CO}-57$ & 3.24E-03 \\
\hline $\mathrm{CO}-58$ & 1. $80 E+00$ \\
\hline FE-59 & 3. $36 \mathrm{E}-02$ \\
\hline $\mathrm{CO}-60$ & $9.50 \mathrm{E}-01$ \\
\hline NI -65 & $0.00 E+00$ \\
\hline $\mathrm{ZN}-63$ & $2.35 E-04$ \\
\hline SE-75 & 2.68E-04 \\
\hline$K R-85$ & $8.15 \mathrm{E}-02$ \\
\hline $\mathbf{K R}-\mathbf{8 5 M}$ & $8.06 \mathrm{E}-05$ \\
\hline $\mathbf{K R}-\mathbf{8 7}$ & 4. 53E-06 \\
\hline $\mathbf{K R}-88$ & $1.60 \mathrm{E}-03$ \\
\hline SR-89 & 4. $16 E-03$ \\
\hline SR-90 & $1.01 \mathrm{E}-03$ \\
\hline SR-91 & $7.50 E-05$ \\
\hline N/A=NOT APPLICABLE & \\
\hline N/D=NOT DETECTED & \\
\hline N/R=NOT REPORTED & \\
\hline
\end{tabular}


EFFLUENT AND WASTE DISPOSAL ANNUAL REPORT FOR YEAR 1981 AIRBORNE AND LIQUID EFFLUENTS

$\begin{array}{lc}\text { LIQUID EFFLUENTS } & \\ \text { NUCLIDES RELEASED } & \text { ACTIVITY (CI) } \\ \text { SR-92 } & 6.13 E-04 \\ \text { NB-95 } & 8.60 E-02 \\ \text { ZR-95 } & 4.65 E-02 \\ \text { NB-97 } & 7.18 E-02 \\ \text { ZR-97 } & 4.29 E-03 \\ \text { MO-99 } & 3.89 E-04 \\ \text { RU-103 } & 2.17 E-02 \\ \text { RU-106 } & 5.83 E-02 \\ \text { AG-110M } & 1.46 E-01 \\ \text { I-131 } & 1.13 E-01 \\ \text { XE-19IM } & 4.80 E-01 \\ \text { BA-139 } & 3.15 E-03 \\ \text { I-133 } & 7.73 E-05 \\ \text { XE-133 } & 5.60 E+01 \\ \text { XE-133M } & 3.60 E-01 \\ \text { CS-134 } & 6.30 E-01 \\ \text { I-134 } & 3.95 E-05 \\ \text { XE-135 } & 5.75 E-02 \\ \text { CS-196 } & 4.70 E-04 \\ \text { CS-137 } & 1.24 E+00 \\ \text { CS-138 } & 1.18 E-05 \\ \text { BA-140 } & 9.93 E-04 \\ \text { LA-140 } & 8.64 E-02 \\ \text { CE-144 } & 3.48 E-02 \\ \text { EU-152 } & 1.35 E-05 \\ \text { W-187 } & 1.13 E-04 \\ \text { NP-239 } & 1.56 E-04 \\ & \\ \text { TRITIUM } & \\ \text { TOTAL AIRBORNE RELEASE } & 1.25 E+02 \\ \text { TOTAL LIQUID RELEASE } & 4.42 E+02 \\ & \\ \text { VOLUME OF LIQUID WASTE RELEASED (PRIOR TO DILUTION) } \\ \text { VOLUME OF DILUTION WATER USED DURING PERIOD } \\ \end{array}$

N/A $=$ NOT APPLICABLE

$N / D=$ NOT DETECTED

N/R $=$ NOT REPORTED 
EFFLUENT AND WASTE DISPOSAL ANNUAL REPORT FOR YEAR 1981 AIRBORNE AND LIQUID EFFLUENTS

UNIT NUMBER: 2

TYPE : PWR

DOCKET NO . : 50-368

COOLING WATER SOURCE : COOLING TOWER
LICENSEE : ARKANSAS POWER \& LIGHT

LICENSED POWER (MWT) : 2815.00

INITIAL CRITICALITY: $12 / 05 / 78$
ACTIVITY (CI)

2.72E-06

$1.90 \mathrm{E}-05$

1. $97 \mathrm{E}-05$

1. 74E-07

$2.21 \mathrm{E}-05$

4 . $10 E-06$

1. $99 \mathrm{E}-07$

2.70E+00

6. $50 \mathrm{E}+00$

$0.00 \mathrm{E}+00$

4. 10E+00

7.37E-06

1. . 90E-06

6. $94 \mathrm{E}-06$

1. 62E-06

1. 14E-07

4. 14E-07

1. $91 \mathrm{E}-06$

1. 85E-07

4. $32 E-08$

1. 37 E-02

3. $90 \mathrm{E}+01$

2.02E-07

3. 53E-04

4. $03 \mathrm{E}+03$

1. $90 \mathrm{E}+00$

2. 02E-06

6. 14E-06

2.75E+02

4. 34E-06

1. 28E-06

$6.62 \mathrm{E}-05$

4. 23E-06

1. $98 \mathrm{E}-07$

$6.54 \mathrm{E}-05$

ACTIVITY (CI)

$1.45 \mathrm{E}-03$

1. 85E-03

1. $19 \mathrm{E}-01$

2. 55E-02

5. $37 \mathrm{E}-04$

4. 40E-01

N/A=NOT APPLICABLE

$N / D=N O T$ DETECTED

N/R=NOT REPORTED 
EFFLUENT AND WASTE DISPOSAL ANNUAL REPORT FOR YEAR 1981 AIRBORNE AND LIQUID EFFLUENTS

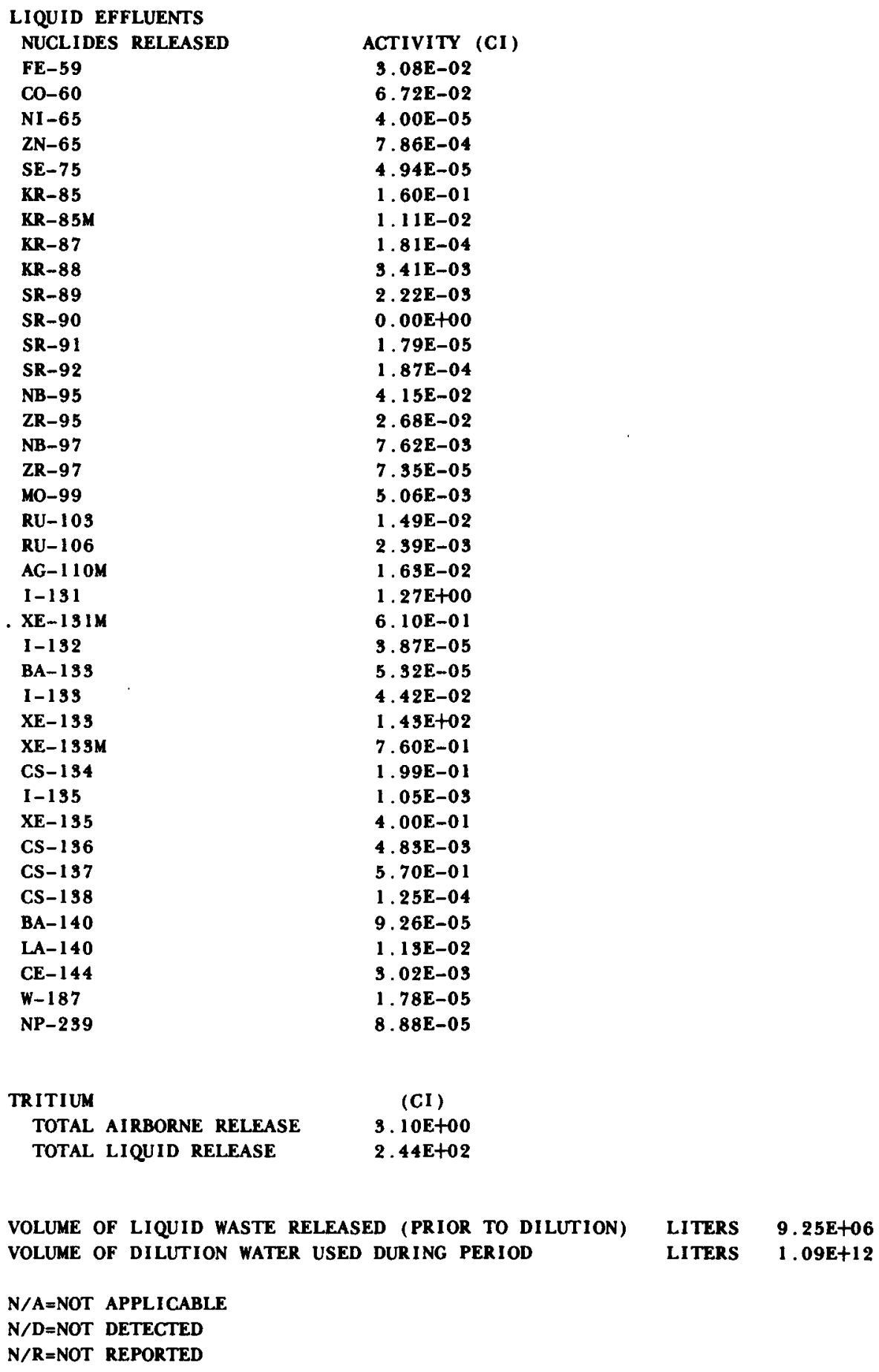


EFFLUENT AND WASTE DISPOSAL ANNUAL REPORT FOR YEAR 1981 AIRBORNE AND LIQUID EFFLUENTS

UNIT NUMBER: 1

TYPE :

PWR

DOCKET NO , : 50-334

COOLING WATER SOURCE:OHIO RIVER

\section{AIRBORNE EFFLUENTS}

NUCL.IDES RELEASED

MN-54

CO-58

CO-60

$\mathrm{KR}-85$

KR-85M

KR-87

$K R-88$

SR-89

SR-90

I- 131

$\mathrm{XE}-131 \mathrm{M}$

I-133

$\mathrm{XE}-133$

XE-1 133M

CS-134

I-135

$\mathrm{XE}-135$

$\mathrm{XE}-135 \mathrm{M}$

CS- 137

XE-1 38

BA-LA- 140

LIQUID EFFLUENTS

NUCLIDES RELEASED

CR-5 I

MN -54

CO-58

FE-59

$\mathrm{CO}-60$

$\mathrm{ZN}-65$

SR-89

SR-90

ZR-NB-95

MO-99

TC-99M

I-131

I- 133

$\mathrm{XE}-133$

CS-134

$\mathrm{XE}-135$

CS-137

BA- 140

LA- 140

CE-141
LICENSEE : DUQUESNE LIGHT + OHIO EDISON + PENNSYLVANIA POWER LICENSED POWER (MWT) :2652.00 INITIAL. CRITICALITY: 05/10/76

N/A=NOT APPLICABLE $N / D=N O T$ DETECTED $N / R=$ NOT REPORTED

ACTIVITY (CI)

4. 43E-07

$1.50 \mathrm{E}-05$

$5.52 \mathrm{E}-05$

$<6.58 \mathrm{E}+00$

$<4.50 \mathrm{E}-01$

$<1.17 \mathrm{E}-01$

$<1.94 \mathrm{E}-01$

$<1.27 E-05$

$<1.02 E-04$

$<6.41 \mathrm{E}-03$

$8.33 \mathrm{E}+00$

$<2.77 \mathrm{E}-04$

$<7.82 E+02$

4. $06 E+00$

$<1.03 \mathrm{E}-07$

$<9.28 \mathrm{E}-05$

$<3.77 \mathrm{E}+00$

$<1.76 \mathrm{E}-01$

$<5.16 \mathrm{E}-05$

$<6.64 \mathrm{E}-07$

<1.62E-07

ACTIVITY (CI)

$<2.84 E-05$

6. $05 \mathrm{E}-03$

2. 50E-02

$<2.09 \mathrm{E}-06$

8. 99E-02

$<6.60 \mathrm{E}-07$

5. 40E-04

1.07E-05

$<8.90 E-04$

$<7.69 \mathrm{E}-07$

$<3.06 E-06$

2. 90E-03

1. 65E-04

3. $07 \mathrm{E}-02$

5. 10E-03

$<1.63 E-04$

1. 33E-02

$\langle 2.64 \mathrm{E}-07$

$<1.24 E-07$

$<4.11 E-07$ 
EFFLUENT AND WASTE DISPOSAL ANNUAL REPORT FOR YEAR 1981 AIRBORNE AND LIQUID EFFLUENTS

\begin{abstract}
LIQUID EFFLUENTS
NUCLIDES RELEASED
\end{abstract}

TRITIUM

TOTAL AIRBORNE RELEASE

TOTAL LIQUID RELEASE
ACTIVITY (CI)

(CI)

9.49E-02

$1.40 E+02$

VOLUME OF LIQUID WASTE RELEASED (PRIOR TO DILUTION)

LITERS 4.30E+06

VOLUME OF DILUTION WATER USED DURING PERIOD

LITERS $4.29 E+09$ 
EFFLUENT AND WASTE DISPOSAL ANNUAL REPORT FOR YEAR 1981 SOLID EFFLUENTS

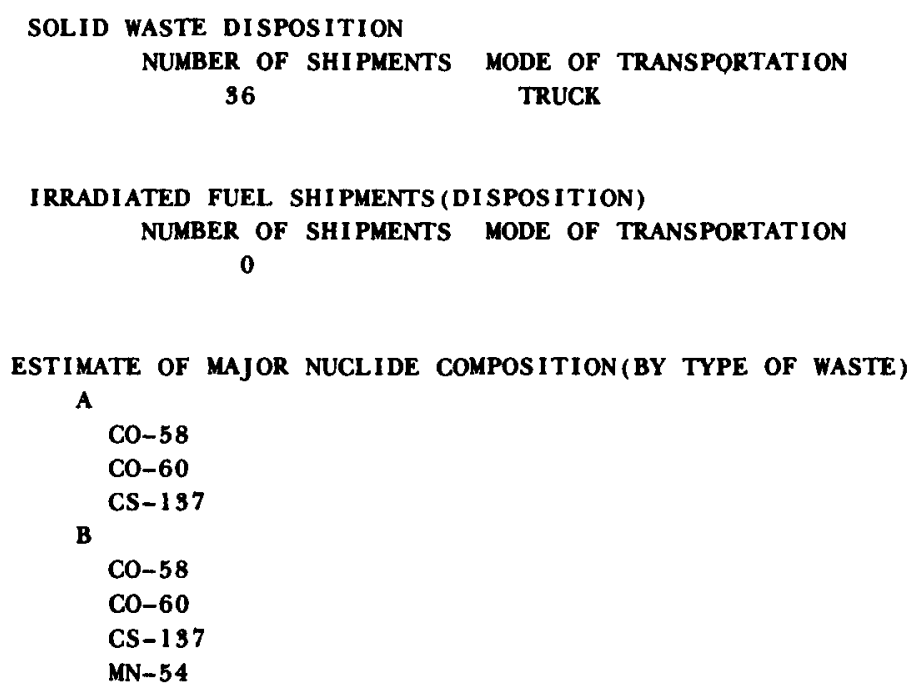

$\begin{array}{ccc} & \text { JAN-JUNE } & \text { JULY-DEC } \\ & & \\ \% & 2.26 E+01 & 1.16 E+01 \\ \% & 6.65 E+01 & 6.92 E+01 \\ \% & 4.40 E+00 & 9.10 E+00 \\ & & \\ \% & 2.26 E+01 & 9.40 E+00 \\ \% & 6.09 E+01 & 8.58 E+01 \\ \% & & 2.70 E+00\end{array}$

TYPE OF WASTE
A. SPENT RESINS, FILTER SLUDGES, EVAPORATOR BOTTOMS, ETC.
B. DRY COMPRESSIBLE WASTE, CONTAMINATED EQUI PMENT, ETC.
C. IRRADIATED COMPONENTS, CONTROL RODS, ETC.
D. OTHER

$\begin{array}{lc}\text { UNIT } & \text { YEAR TOTAL } \\ \text { M3 } & 1.07 E+02 \\ \text { CI } & 8.73 E+01 \\ \text { M3 } & 1.06 E+02 \\ \text { CI } & 5.68 E+00 \\ \text { M3 } & \\ \text { CI } & \\ \text { M3 } & \\ \text { CI } & \end{array}$


EFFLUENT AND WASTE DISPOSAL ANNUAL REPORT FOR YEAR 1981 AIRBORNE AND LIQUID EFFLUENTS

UNIT NUMBER : 1

TYPE: $\quad$ BWR

DOCKET NO. : 50-155

COOLING WATER SOURCE: LAKE MICHIGAN

\section{AIRBORNE EFFLUENTS}

NUCLIDES RELEASED

$\mathrm{N}-13$

NA-24

CR-5 1

MN-54

CO-60

BR-82

KR-83M

KR-85

KR-85M

KR-87

KR-88

KR-89

KR-90

KR-9 1

I-131

$\mathrm{XE}-131 \mathrm{M}$

I- 133

XE-133

$\mathrm{XE}-133 \mathrm{M}$

I-135

XE-135

$\mathrm{XE}-135 \mathrm{M}$

CS-136

CS -137

$\mathrm{XE}-137$

$\mathrm{XE}-138$

$\mathrm{XE}-139$

BA-140

LA- 140

XE-140

UNIDENTIFIED

LIQUID EFFLUENTS

NUCLIDES RELEASED

CR-5 1

$\mathrm{MN}-54$

CO-58

FE-59

CO-60

$\mathrm{ZN}-65$

SR-89

SR-90

AG- $110 \mathrm{M}$

SB-1 24

N/A $=$ NOT APPLICABLE

$N / D=N O T$ DETECTED

N/R=NOT REPORTED
LICENSEE : CONSUMERS POWER

LICENSED POWER (MWT) : 240.00

INITIAL CRITICALITY: 09/27/62
ACTIVITY (CI)

1. $81 E+03$

8. 38E-04

5. $42 \mathrm{E}-04$

4. 99E-05

2. 10E-04

1. 25E-02

$3.09 \mathrm{E}+02$

3. $63 \mathrm{E}+00$

3. $67 \mathrm{E}+02$

$1.36 E+03$

$9.53 \mathrm{E}+02$

8.53E+02

$9.55 \mathrm{E}+02$

1. $19 \mathrm{E}+01$

2. 38E-03

1. 34E+00

3. 39E-02

2.85E+02

7. 74E+00

2. 79E-02

1. 30E+03

1. 39E+03

4. $60 \mathrm{E}-05$

1. 50E-04

1. 35E+03

$7.34 \mathrm{E}+03$

1. $27 \mathrm{E}+03$

1. 45E-03

$9.55 \mathrm{E}-03$

1. $29 E+02$

1. 28E-03

ACTIVITY (CI)

1. $91 \mathrm{E}-02$

1. 69E-02

2. 25E-04

2.37E-03

4. 99E-02

1. 82E-04

$6.87 \mathrm{E}-04$

1. $02 E-03$

1. 70E-04

1. 37E-04 
EFFLUENT AND WASTE DISPOSAL ANNUAL REPORT FOR YEAR 1981

AIRBORNE AND LIQUID EFFLUENTS

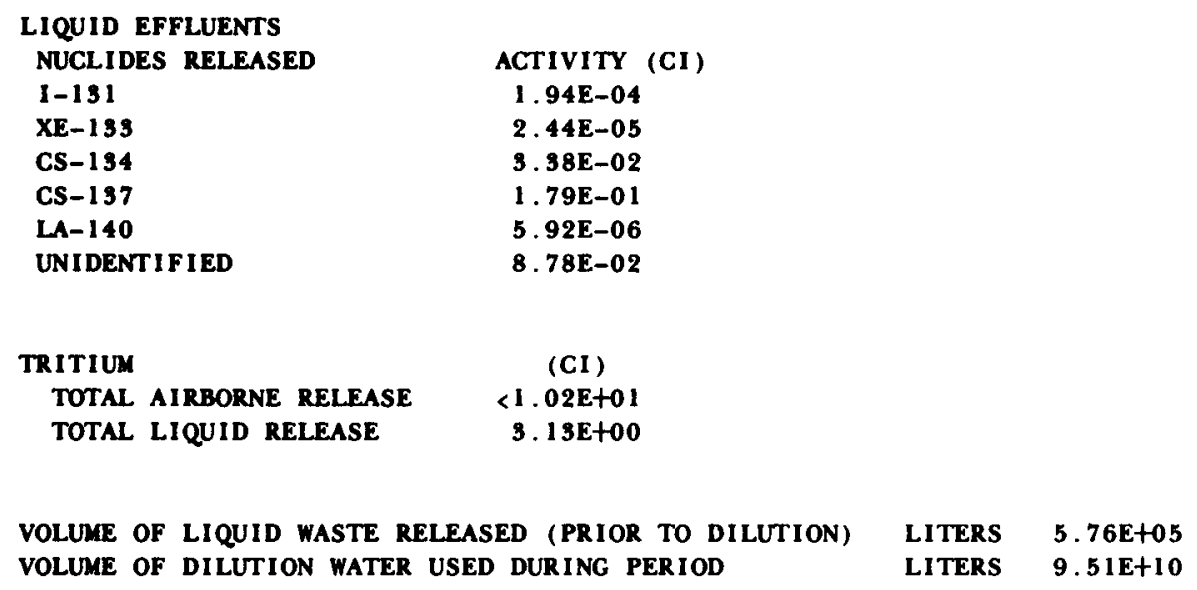

N/A=NOT APPLICABLE

$N / D=N O T$ DETECTED

N/R=NOT REPORTED 
INSTALLATION:BIG ROCK POINT

EFFLUENT AND WASTE DISPOSAL ANNUAL REPORT FOR YEAR 1981 SOLID EFFLUENTS

SOLID WASTE DISPOSITION NUMBER OF SHIPMENTS MODE OF TRANSPORTATION 10 TRUCK

4 TRUCK

IRRADIATED FUEL SHI PMENTS (DISPOS ITION) NUMBER OF SHIPMENTS MODE OF TRANSPORTATION o

DESTINATION

TYPE OF WASTE

A. SPENT RESINS, FILTER SLUDGES, EVAPORATOR BOTTOMS, ETC.

B. DRY COMPRESSIBLE WASTE, CONTAMINATED EQUI PMENT, ETC.

C. IRRADIATED COMPONENTS, CONTROL RODS, ETC.

DESTINATION

BARNWELL, S.C.

RICHLAND, WA

D. OTHER

UNIT YEAR TOTAL

M3 4.04E+O 1

CI $\quad 3.05 E+02$

M3 9.84E+OI

CI 4.44E+00

M3 5.66E +00

CI 8.00E+00

N/A=NOT APPLICABLE

N/D=NOT DETECTED

N/R=NOT REPORTED 
EFFLUENT AND WASTE DISPOSAL ANNUAL REPORT FOR YEAR 1981 AIRBORNE AND LIQUID EFFLUENTS

UNIT NUMBER : 1

TYPE : BWR

DOCKET NO . : 50-259

COOLING WATER SOURCE:TENNESSEE RIVER UNIT NUMBER : 2

TYPE : BWR

DOCKET NO . : 50-260

COOLING WATER SOURCE : TENNESSEE RIVER

UNIT NUMBER : 3

TYPE : BWR

DOCKET NO . : 50-296

COOLING WATER SOURCE:TENNESSEE RIVER

AIRBORNE EFFLUENTS

NUCLIDES RELEASED

H-3

AR-4 1

MN-54

$\mathrm{CO}-58$

FE -59

CO-60

KR-85

KR-85M

KR-87

KR-88

SR-89

SR-90

NB-95

ZR-95

I- 131

$M I-131$

I -133

MI-133

XE-1 13

CS-134

I-135

MI -135

XE-1 135

XE-135M

CS-137

$\mathrm{XE}-138$

BA-140

LA-140

\section{LIQUID EFFLUENTS}

NUCLIDES RELEASED

H-3

NA-24

CR-5 1

MN-54

MN-56
ACTIVITY (CI)

3. 80E+01

4. $49 \mathrm{E}+02$

3. 45E-03

2.53E-03

5. 59E-03

1. 70E-02

8. $42 E+02$

2. $67 \mathrm{E}+03$

1. $01 \mathrm{E}+03$

1. $22 \mathrm{E}+04$

3. 08E-03

3. 21 E-03

2.67E-03

8. 16E-03

4. 00E-02

4. 00E-02

1. $50 \mathrm{E}-02$

1. 50E-02

9. $35 E+03$

3. 76E-03

3. 73E-02

3. $73 E-02$

5. 48E+02

1. $84 \mathrm{E}+03$

4. 33E-03

1. $62 \mathrm{E}+04$

3. 43E-03

1. 05E-02

ACTIVITY (CI)

2. 42E+01

9. 22E-02

2. 34E-01

7. 45E-02

2.92E-02
LICENSEE : TENNESSEE VALLEY AUTHORITY

LICENSED POWER (MWT) : 3293.00

INITIAL CRITICALITY:08/17/73

LICENSEE : TENNESSEE VALLEY AUTHORITY

LICENSED POWER (MWT) : $\mathbf{3 2 9 3 . 0 0}$

INITIAL CRITICALITY:07/20/74

LICENSEE : TENNESSEE VALLEY AUTHORITY

LICENSED POWER (MWT) :3293.00

INITIAL CRITICALITY :08/08/76

N/A=NOT APPLICABLE

$N / D=N O T$ DETECTED

N/R=NOT REPORTED 
EFFLUENT AND WASTE DISPOSAL ANNUAL REPORT FOR YEAR 1981 AIRBORNE AND LIQUID EFFLUENTS

LIQUID EFFLUENTS
NUCLIDES RELEASED
CO-58
FE-59
CO-60
CU-64
ZN-65
BR-82
SR-89
SR-90
NB-95
ZR-95
MO-99
TC-99M
AG-110M
SB-124
SB-125
I-131
I-133
XE-133
CS-134
I-135
XE-135
CS-136
CS-137
BA-140
LA-140
CE-141
SM-153

ACTIVITY (CI)
$1.99 E-02$
$1.54 E-01$
$3.48 E-01$
$1.43 E-01$
$3.79 E-01$
$1.14 E-02$
$5.50 E-02$
$2.72 E-02$
$1.72 E-02$
$1.72 E-02$
$4.73 E-03$
$4.73 E-03$
$3.02 E-02$
$9.37 E-03$
$1.21 E-02$
$3.96 E-02$
$1.45 E-02$
$6.90 E-02$
$1.38 E-01$
$7.56 E-03$
$6.56 E-02$
$7.49 E-03$
$1.95 E-01$
$2.58 E-03$
$2.58 E-03$
$2.94 E-02$
$1.16 E-03$


EFFLUENT AND WASTE DISPOSAL ANNUAL REPORT FOR YEAR 1981 AIRBORNE AND LIQUID EFFLUENTS

UNIT NUMBER: 1

TYPE: $\quad$ BWR

DOCKET NO. : 50-325

COOLING WATER SOURCE : CAPE FEAR RIVER UNIT NUMBER : 2

TYPE : $\quad$ BWR

DOCKET NO. : 50-324

COOLING WATER SOURCE:CAPE FEAR RIVER

\section{AIRBORNE EFFLUENTS}

NUCLI DES RELEASED

AR-4 1

CR-51

MN-54

CO-57

C0-58

FE-59

CO-60

$\mathrm{ZN}-65$

KR-85

KR-85M

SR-85

KR-87

KR-88

ZR-NB-95

MO-99

TC-99M

RU- 103

RH-106

AG- $110 \mathrm{M}$

SN-117M

I-131

1-132

I-133

XE-133

XE-1 33M

CS-134

1-134

I-135

$\mathrm{XE}-135$

XE-135M

CS-136

CS-137

$\mathrm{XE}-138$

BA- 139

BA-LA-1 40

CE- 141

CE-144

NP-239

UNIDENTIFIED
LICENSEE : CAROLINA POWER \&LIGHT LICENSED POWER (MWT) : 2436.00 IN ITIAL. CRITICALITY : 10/08/76

LICENSEE : CAROLINA POWER \&LIGHT LICENSED POWER (MWT) :2436.00 INITIAL CRITICALITY : 03/20/75

N/A=NOT APPLICABLE

$N / D=N O T$ DETECTED

N/R=NOT REPORTED

ACTIVITY (CI)
$2.76 E+03$
$3.78 E-02$
$2.06 E-03$
$2.79 E-08$
$3.47 E-04$
$1.27 E-04$
$3.38 E-03$
$3.75 E-06$
N/D
$2.64 E+04$
$2.79 E-07$
$5.73 E+04$
$5.95 E+04$
$1.62 E-06$
$1.65 E-04$
$1.52 E-03$
$2.56 E-07$
$4.42 E-04$
$1.04 E-05$
$3.37 E-05$
$2.66 E-01$
$9.81 E-02$
$9.99 E-01$
$7.83 E+04$
$2.88 E+03$
$8.67 E-04$
$6.36 E-02$
$8.48 E-01$
$9.75 E+04$
$3.54 E+04$
NDD
$2.05 E-03$
$1.40 E+05$
$2.71 E-03$
$3.32 E-03$
$2.72 E-05$
$1.47 E-06$
$2.00 E-05$
$2.21 E+04$


EFFLUENT AND WASTE DISPOSAL ANNUAL REPORT FOR YEAR 1981 AIRBORNE AND LIQUID EFFLUENTS

\begin{tabular}{l} 
LIQUID EFFLUENTS \\
NUCLIDES RELEASED \\
F-18 \\
NA-24 \\
AR-41 \\
CR-51 \\
MN-54 \\
MN-56 \\
CO-57 \\
CO-58 \\
FE-59 \\
CO-60 \\
CU-64 \\
NI-65 \\
ZN-65 \\
AS-76 \\
KR-85 \\
KR-85M \\
SR-85 \\
SR-89 \\
SR-90 \\
SR-91 \\
Y-91M \\
SR-92 \\
ZR-NB-95 \\
NB-97 \\
NB-97M \\
ZR-97 \\
MO-99 \\
TC-99M \\
TC-101 \\
RU-103 \\
AG-110M \\
IN-119M \\
SN-113 \\
SN-117M \\
SB-122 \\
TE-129M \\
I-131 \\
XE-131M \\
I-132 \\
TE-132 \\
I-133 \\
XE-133 \\
XE-133M \\
CS-134 \\
I-134 \\
XE-135 \\
\\
\hline
\end{tabular}

$$
\begin{aligned}
& \text { ACTIVITY (CI) } \\
& 3.15 E-02 \\
& 2.50 E-01 \\
& 3.80 E-04 \\
& 4.31 E-01 \\
& 2.21 E-01 \\
& 4.84 E-05 \\
& 1.65 E-05 \\
& 4.15 E-02 \\
& 1.01 E-02 \\
& 4.68 E-01 \\
& 4.51 E-02 \\
& \text { N/D } \\
& 1.58 E-03 \\
& 9.25 E-04 \\
& 1.16 E-02 \\
& 4.06 E-05 \\
& 4.68 E-05 \\
& 1.02 E-02 \\
& 1.36 E-03 \\
& 1.10 E-03 \\
& 3.65 E-04 \\
& 4.58 E-04 \\
& 5.57 E-05 \\
& 7.49 E-04 \\
& 2.08 E-06 \\
& \text { 3.50E-05 } \\
& \text { N/D } \\
& 3.11 E-03 \\
& 1.56 E-05 \\
& 6.88 E-05 \\
& 1.51 E-03 \\
& \text { N/D } \\
& \text { N/D } \\
& 1.01 E-05 \\
& 1.60 E-02 \\
& \text { N/D } \\
& 9.40 E-02 \\
& 1.21 E-02 \\
& 1.75 E-04 \\
& 2.55 E-03 \\
& 2.71 E-02 \\
& 2.35 E-01 \\
& 3.62 E-03 \\
& 1.71 E-01 \\
& 1.52 E-05 \\
& 6.25 E-04 \\
& 1.18 E-01 \\
& 1.33 E-03 \\
&
\end{aligned}
$$

N/A=NOT APPLICABLE $N / D=N O T$ DETECTED N/R=NOT REPORTED 
EFFLUENT AND WASTE DISPOSAL ANNUAL REPORT FOR YEAR 1981 AIRBORNE AND LIQUID EFFLUENTS

$\begin{array}{ll}\text { LIQUID EFFLUENTS } & \\ \text { NUCLIDES RELEASED } & \text { ACTIVITY } \\ \text { CS-136 } & 1.79 E-03 \\ \text { CS-137 } & 2.55 E-01 \\ \text { CS-138 } & 2.15 E-06 \\ \text { BA-139 } & 8.59 E-04 \\ \text { CE-139 } & \text { N/D } \\ \text { BA-LA-140 } & 2.65 E-02 \\ \text { CE-141 } & 9.86 E-05 \\ \text { CE-144 } & 9.72 E-04 \\ \text { W-187 } & \text { N/D } \\ \text { NP-239 } & 1.29 E-02 \\ \text { UNIDENTIFIED } & 6.51 E-02 \\ & \\ \text { TRITIUM } & \\ \text { TOTAL AIRBORNE RELEASE } & 1.76 E+01 \\ \text { TOTAL LIQUID RELEASE } & 2.26 E+01\end{array}$

VOLUME OF LIQUID WASTE RELEASED (PRIOR TO DILUTION) LITERS 6.20E+07 VOLUME OF DILUTION WATER USED DURING PERIOD

LITERS $1.63 \mathrm{E}+11$

N/A=NOT APPLICABLE

N/D=NOT DETECTED

N/R=NOT REPORTED 
INSTALLATION : BRUNSWICK

EFFLUENT AND WASTE DISPOSAL ANNUAL REPORT FOR YEAR 1981 SOLID EFFLUENTS

SOLID WASTE DISPOSITION NUMBER OF SHIPMENTS $\mathbf{3 1 8}$ 56 MODE OF TRANSPORTATION SOLE USE VEHICLE SOLE USE VEHICLE

I RRADIATED FUEL SHI PMENTS (DISPOSITION) NUMBER OF SHIPMENTS MODE OF TRANSPORTATION 0

ESTIMATE OF MAJOR NUCLIDE COMPOSITION(BY TYPE OF WASTE) A

BA-140

Co-58

CO-60

CR-5 1

CS-134

CS-137

FE-59

I -131

I - 133

LA-140

MN-54

MO-99

NA-24

NB-97

SB-1 22

SR-92

TC-99M

TE-1 31

ZN-65

$\mathrm{ZN}-69 \mathrm{M}$

B

BA- 140

CO-58

CO-60

CR-5 1

CS-134

CS-197

FE-59

I - 18 I

I-133

LA-140

MN-54

MO-99

NA-24

NB-97

SB-1 22

N/A $=$ NOT APPLICABLE

$N / D=N O T$ DETECTED

N/R $=$ NOT REPORTED
DESTINATION

BARNWELL, S.C.

RICHLAND, WA

DESTINATION

JAN-JUNE JULY-DEC

7.59E-01

2.94E+01 3.16E+01

2.44E+01 3.28E-01

$6.68 \mathrm{E}+00 \quad 5.37 \mathrm{E}+00$

$1.09 \mathrm{E}+01 \quad 8.46 \mathrm{E}+00$

$2.44 E+00 \quad 6.65 E-01$

1.85E-01 2.52E+00

4. 56E-02

$1.02 E+00$

2.07E+01 1.35E-01

6. 97E-04

2.46E-02 1.25E-01

1. $13 \mathrm{E}-03$

$8.45 \mathrm{E}-01 \quad 7.56 \mathrm{E}-01$

$1.02 \mathrm{E}-01$ 1.01E-04

9. 88E-02

5.30E-02

3.36E-01 1.85E-01

8.37E-06

4.00E+00

7. 59E-01

2.00E+00

2.94E+01 3.16E+01

2.44E+01 3.28E-01

$6.68 \mathrm{E}+00 \quad 5.37 \mathrm{E}+00$

1.09E+01 8.46E+00

$2.44 E+00 \quad 6.65 E-01$

I.85E-01 2.52E+00

4. 56E-02

$1.02 E+00$

2.07E+01 1.35E-01

6.97E-04

$2.46 \mathrm{E}-02 \quad 1.25 \mathrm{E}-01$

1. 13E-03

8.45E-01 7.56E-01 
INSTALLATION : BRUNSWICK

EFFLUENT AND WASTE DISPOSAL ANNUAL REPORT FOR YEAR 1981 SOLID EFFLUENTS

ESTIMATE OF MAJOR NUCLIDE COMPOSITION(BY TYPE OF WASTE) SR-92

TC-99M

TE-131

$\mathrm{ZN}-65$

$\mathrm{ZN}-69 \mathrm{M}$

$\begin{array}{ccc} & \text { JAN-JUNE } & \text { JULY-DEC } \\ \% & 1.02 E-01 & 1.01 E-04 \\ \% & & 9.88 E-02 \\ \% & & 5.30 E-02 \\ \% & 3.36 E-01 & 1.85 E-01 \\ \$ & & 8.37 E-06\end{array}$

TYPE OF WASTE

A. SPENT RES INS, FILTER SLUDGES, EVAPORATOR BOTTOMS , ETC.

B. DRY COMPRESS IBLE WASTE, CONTAMINATED EQUI PMENT, ETC.

UNIT YEAR TOTAL

MS $\quad 1.37 E+03$

CI $7.41 \mathrm{E}+03$

M3 2.93E+03

CI 6.03E+OI

C. IRRADIATED COMPONENTS, CONTROL RODS, ETC

M3

CI

D. OTHER 
EFFLUENT AND WASTE DISPOSAL ANNUAL REPORT FOR YEAR 1981 AIRBORNE AND LIQUID EFFLUENTS

UNIT NUMBER : 1

TYPE: PWR

DOCKET NO. : 50-317

COOLING WATER SOURCE:CHESAPEAKE BAY UNIT NUMBER : 2

TYPE : PWR

DOCKET NO. : 50-318

COOLING WATER SOURCE : CHESAPEAKE BAY

\section{AIRBORNE EFFLUENTS}

NUCLIDES RELEASED

AR-4 1

CO-58

CO-60

BR-82

KR-85M

KR-87

KR-88

RB-88

SR-89

SR-90

I -131

$\mathrm{XE}-131 \mathrm{M}$

I- 132

I- 133

XE-193

XE-1 39M

CS-134

I-195

XE-135

CS-137

CS-138

BA-139

CE-1 199

BA-140

LA-140

CE-141

LIQUID EFFLUENTS

NUCLIDES RELEASED

CR-51

$M N-54$

MN -56

CO-57

CO-58

CO-60

SR-89

SR-90

NB-95

ZR-95

ZR-97

N/A=NOT APPLICABLE $N / D=N O T$ DETECTED N/R=NOT REPORTED
LICENSEE : BALTIMORE GAS \& ELECTRIC LICENSED POWER (MWT) :2700.00 INITIAL CRITICALITY : 10/07/74

LICENSEE : BALTIMORE GAS \& ELECTRIC LICENSED POWER (MWT): 2700.00 INITIAL CRITICALITY: $11 / 30 / 76$

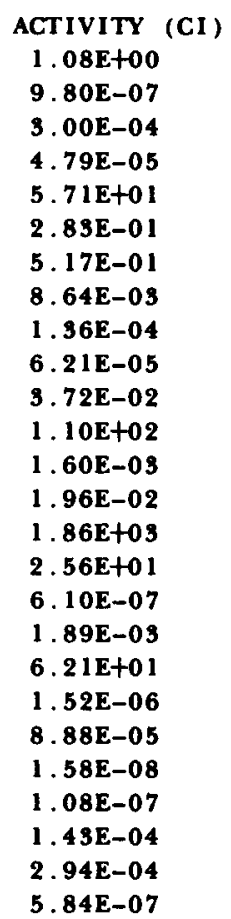


EFFLUENT AND WASTE DISPOSAL ANNUAL REPORT FOR YEAR 1981 AIRBORNE AND LIQUID EFFLUENTS

$\begin{array}{lc}\text { LIQUID EFFLUENTS } & \\ \text { NUCLIDES RELEASED } & \text { ACTIVITY (CI) } \\ \text { MO-99 } & 1.66 \mathrm{E}-03 \\ \text { RU-103 } & 4.45 \mathrm{E}-04 \\ \text { RU-106 } & 1.07 \mathrm{E}-02 \\ \text { AG-110M } & 1.62 \mathrm{E}-02 \\ \text { SN-113 } & 3.26 \mathrm{E}-03 \\ \text { SB-124 } & 2.86 \mathrm{E}-04 \\ \text { SB-125 } & 7.58 \mathrm{E}-02 \\ \text { I-131 } & 3.07 \mathrm{E}-01 \\ \text { XE-131M } & 2.68 \mathrm{E}-02 \\ \text { I-133 } & 5.89 \mathrm{E}-02 \\ \text { XE-133 } & 3.91 \mathrm{E}+00 \\ \text { XE-133M } & 1.49 \mathrm{E}-02 \\ \text { CS-134 } & 5.07 \mathrm{E}-02 \\ \text { XE-135 } & 3.00 \mathrm{E}-02 \\ \text { CS-137 } & 1.03 \mathrm{E}-01 \\ \text { BA-140 } & 4.03 \mathrm{E}-03 \\ \text { LA-140 } & 3.77 \mathrm{E}-02 \\ \text { CE-141 } & 6.58 \mathrm{E}-06 \\ \text { CE-144 } & 2.87 \mathrm{E}-03 \\ \text { UNIDENTIFIED } & 9.38 \mathrm{E}-03 \\ & \\ \text { TRITIUM } & \\ \text { TOTAL AIRBORNE RELEASE } & 5.81 \mathrm{E}+00 \\ \text { TOTAL LIQUID RELEASE } & 1.00 \mathrm{E}+03\end{array}$

VOLUME OF LIQUID WASTE RELEASED (PRIOR TO DILUTION) LITERS 3.87E+07 VOLUME OF DILUTION WATER USED DURING PERIOD 
EFFLUENT AND WASTE DISPOSAL ANNUAL REPORT FOR YEAR 1981 SOLID EFFLUENTS

SOLID WASTE DISPOSITION
NUMBER OF SHIPMENTS MODE OF TRANSPORTATION
42
MOTOR SURFACE TRANS I

ESTIMATE OF MAJOR NUCLIDE COMPOSITION(BY TYPE OF WASTE)

A

C0-58

C0-60

CS-134

CS-137

MN-54

SB-122

B

C0-58

C0-60

CS-134

CS-137

MN-54

NB-95

C

C0-58

CO-60

CR-5 1

CS-134

CS-137

MN-54

NB-95

SB-1 22

SB-1 25

ZR-95
DESTINATION

BARNWELL, SC
JAN-JUNE JULY-DEC

\begin{tabular}{|c|c|c|}
\hline ж & $2.52 E+01$ & $3.48 \mathrm{E}+01$ \\
\hline$\%$ & 1. $60 E+01$ & $5.80 \mathrm{E}+00$ \\
\hline 5 & $1.87 E+01$ & $2.03 \mathrm{E}+01$ \\
\hline & $2.34 \mathrm{E}+01$ & $2.97 \mathrm{E}+01$ \\
\hline & $1.61 \mathrm{E}+01$ & 4. $70 E+00$ \\
\hline & & $1.12 \mathrm{E}+00$ \\
\hline & $1.60 E+01$ & $2.63 E+01$ \\
\hline & $7.23 \mathrm{E}+01$ & 1.74E+01 \\
\hline$\%$ & $2.26 E+00$ & 1.06E +01 \\
\hline$\%$ & $6.38 E+00$ & $4.13 \mathrm{E}+01$ \\
\hline$\%$ & $1.66 \mathrm{E}+00$ & $2.60 \mathrm{E}+00$ \\
\hline$\%$ & $1.03 \mathrm{E}+00$ & \\
\hline & 1. $72 \mathrm{E}+01$ & $2.18 \mathrm{E}+01$ \\
\hline & $2.21 \mathrm{E}+01$ & $1.98 \mathrm{E}+01$ \\
\hline & $2.30 E+00$ & \\
\hline & $7.28 E+00$ & \\
\hline & $3.53 \mathrm{E}+01$ & \\
\hline & 3. $79 E+00$ & $3.30 E+00$ \\
\hline & $6.68 \mathrm{E}+00$ & $6.30 \mathrm{E}+00$ \\
\hline & & $2.80 E+00$ \\
\hline & 3. $96 E+00$ & $4.31 \mathrm{E}+01$ \\
\hline & 1. $36 \mathrm{E}+00$ & \\
\hline
\end{tabular}

UNIT YEAR TOTAL

M3 5. 29E+01

CI $9.40 \mathrm{E}+01$

M3 $2.92 E+02$

CI $2.40 \mathrm{E}+00$

M3 $1.56 \mathrm{E}+02$

CI 2.17E+00

TYPE OF WASTE BOTTOMS , ETC.

B. DRY COMPRESS IBLE WASTE, CONTAMINATED EQUI PMENT, ETC.

C. IRRADIATED COMPONENTS, CONTROL RODS , ETC

D. OTHER

N/A=NOT APPLICABLE

$N / D=N O T$ DETECTED

N/R=NOT REPORTED 
EFFLUENT AND WASTE DISPOSAL ANNUAL REPORT FOR YEAR 1981 AIRBORNE AND LIQUID EFFLUENTS

UNIT NUMBER : 1

TYPE:

PWR

DOCKET NO. : 50-315

COOLING WATER SOURCE: LAKE MICHIGAN

UNIT NUMBER : 2

TYPE: $\quad$ PWR

DOCKET NO . : 50-316

COOLING WATER SOURCE: LAKE MICHIGAN

AIRBORNE EFFLUENTS

NUCLIDES RELEASED

NA-24

AR-4 1

MN-54

C0-58

C0-60

KR-85

KR-85M

KR-87

KR-88

NB-95

ZR-95

I-190

I-131

XE-131M

$I-133$

XE-133

$\mathrm{XE}-133 \mathrm{M}$

CS-134

I-135

XE- 135

CS-136

CS-137

CE-139

LIQUID EFFLUENTS

NUCLIDES RELEASED

NA-24

CR-5 1

MN-54

CO-57

C0-58

FE-59

C0-60

$\mathrm{ZN}-65$

SR-89

SR-90

ZR-NB-95

ZR-97

CD-109

AG-1 10M

$N / A=N O T$ APPLICABLE

$N / D=N O T$ DETECTED

N/R=NOT REPORTED
LICENSEE : INDIANA \& MICHIGAN ELECTRIC

LICENSED POWER (MWT) : 3250.00

INITIAL CRITICALITY : $01 / 18 / 75$

LICENSEE : INDIANA \& MICHIGAN ELECTRIC

LICENSED POWER (MWT):3391.00

INITIAL CRITICALITY : 07/01/78
ACTIVITY (CI)

2. 59E-01

2.30E-01

$6.08 \mathrm{E}-05$

8. 30E-04

2.13E-03

4. 50E+00

3. $16 \mathrm{E}+00$

9.94E-01

8.01E-01

1. $38 \mathrm{E}-06$

8. 57E-07

$6.47 \mathrm{E}-06$

4. $66 \mathrm{E}-02$

3. $75 \mathrm{E}+00$

4. 77E-02

2. 22E+04

2.07E+01

3. 06E-03

3. 52E-05

2.47E+02

1. 26E-04

1. 33E-02

1. $99 \mathrm{E}-04$

ACTIVITY (CI)

4. 79E-01

2.82E-02

2.75E-02

3. $77 \mathrm{E}-03$

4. 57E-01

6. $14 \mathrm{E}-04$

2. 26E-01

3. $00 \mathrm{E}-03$

1. $46 \mathrm{E}-03$

8.44E-04

2. 78E-02

2. 23E-03

1.71E-03

6. 50E-02 
EFFLUENT AND WASTE DISPOSAL ANNUAL REPORT FOR YEAR 1981 AIRBORNE AND LIQUID EFFLUENTS

LIQUID EFFLUENTS NUCLIDES RELEASED

$\mathrm{SN}-113$

SB-124

I-191

I-133

CS-134

CS-136

CS-137

CE-139

BA-LA-140

TRITIUM

TOTAL AIRBORNE RELEASE

TOTAL LIQUID RELEASE
ACTIVITY (CI)

4. 33E-04

5. $48 \mathrm{E}-03$

1. $53 \mathrm{E}-01$

5. 67E-02

3.92E-02

5. 27E-03

7. 87E-02

8.05E-05

2.48E-04

(CI)

$5.47 \mathrm{E}+00$

9. 15E+02
VOLUME OF LIQUID WASTE RELEASED (PRIOR TO DILUTION) VOLUME OF DILUTION WATER USED DURING PERIOD
LITERS 3.83E+08

LITERS 2.47E+12

N/A=NOT APPLICABLE

$N / D=$ NOT DETECTED

N/R=NOT REPORTED 
INSTALLATION : DONALD C. COOK

EFFLUENT AND WASTE DISPOSAL ANNUAL REPORT FOR YEAR 1981 SOLID EFFLUENTS

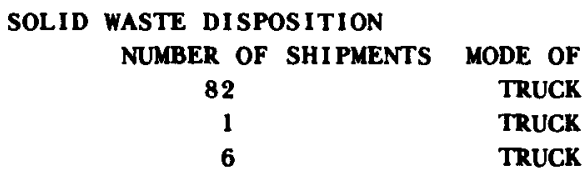

ESTIMATE OF MAJOR NUCLIDE COMPOSITION(BY TYPE OF WASTE)

A

$$
\begin{aligned}
& \text { CO-58 } \\
& \text { CO-60 } \\
& \text { CS-134 } \\
& \text { CS-137 } \\
& \text { CO-58 } \\
& \text { Co-60 } \\
& \text { CS-137 }
\end{aligned}
$$
A. SPENT RESINS, FILTER SLUDGES, EVAPORATOR BOTTOMS , ETC.
B. DRY COMPRESS IBLE WASTE, CONTAMINATED EQUI PMENT, ETC
C. IRRADIATED COMPONENTS, CONTROL RODS, ETC.
D. OTHER

OF WASTE

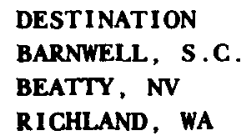

DESTINATION

JAN-JUNE JULY-DEC

$\begin{array}{lll}\$ & 4.50 E+01 & 4.50 E+01 \\ \$ & 2.00 E+01 & 1.00 E+01 \\ \$ & 1.00 E+01 & 1.50 E+01 \\ \% & 2.50 E+01 & 3.00 E+01 \\ & & \\ & 7.00 E+01 & 7.50 E+01 \\ \$ & 5.00 E+00 & 1.00 E+01 \\ \$ & 2.50 E+01 & 1.50 E+01\end{array}$

UNIT YEAR TOTAI

M3 5.19E+02

CI $1.29 \mathrm{E}+03$

M3 $4.45 \mathrm{E}+02$

CI $1.42 \mathrm{E}+02$

M3

CI

M3

CI 
EFFLUENT AND WASTE DISPOSAL ANNUAL REPORT FOR YEAR 1981 AIRBORNE AND LIQUID EFFLUENTS

\begin{abstract}
UNIT NUMBER: I
TYPE: BWR
\end{abstract}

DOCKET NO. : 50-298

COOLING WATER SOURCE :MISSOUR I RIVER

\section{AIRBORNE EFFLUENTS}

NUCLIDES RELEASED

CR-5 1

MN-54

CO-58

CO-60

$\mathrm{ZN}-65$

KR-83M

KR-85

KR-85M

KR-87

KR-88

KR-89

SR-89

SR-90

I-131

XE-131M

1-133

XE- 133

XE-133M

CS-194

I-135

XE- 135

XE-135M

CS-137

$\mathrm{XE}-137$

$X E-138$

BA-LA-140

\section{LIQUID EFFLUENTS}

NUCLIDES RELEASED

NA-24

CR-51

MN-54

MN-56

CO- 58

FE-59

CO-60

$\mathrm{ZN}-65$

SR-89

SR-90

SR-92

ZR-NB-95

MO-99

TC-99M

AG-110M

N/A=NOT APPLICABLE N/D=NOT DETECTED N/R=NOT REPORTED
LICENSEE : NEBRASKA PUBLIC POWER \&IOWA POWER \&LIGHT LICENSED POWER (WWT) :2381.00 INITIAL CRITICALITY:02/21/74

$$
\begin{array}{r}
\text { ACTIVITY (CI) } \\
4.83 E-04 \\
7.35 E-04 \\
1.86 E-04 \\
1.77 E-03 \\
4.65 E-05 \\
5.99 E+01 \\
4.59 E+01 \\
2.40 E+02 \\
6.30 E+03 \\
4.41 E+02 \\
3.02 E+00 \\
1.66 E-04 \\
3.05 E-05 \\
<5.26 E-03 \\
3.55 E+00 \\
<1.18 E-02 \\
4.87 E+02 \\
1.39 E+01 \\
<2.24 E-04 \\
<1.27 E-01 \\
6.71 E+02 \\
4.09 E+01 \\
<3.73 E-04 \\
8.24 E+00 \\
2.11 E+02 \\
<1.66 E-03
\end{array}
$$

ACTIVITY (CI)

$1.85 \mathrm{E}-02$

$<5.25 \mathrm{E}-0\}$

$<6.28 \mathrm{E}-01$

$4.61 \mathrm{E}-04$

$<3.09 E-01$

$<8.77 \mathrm{E}-03$

$<9.76 \mathrm{E}-01$

$<1.98 \mathrm{E}-02$

4. 34E-02

1. 59E-03

$<6.19 E-05$

$<2$. 12E-02

1. 47E-02

<2. 17E-02

< 1.06E-02 
EFFLUENT AND WASTE DJSPOSAL ANNUAL REPORT FOR YEAR 1981 AIRBORNE AND LIQUID EFFLUENTS

$\begin{array}{lc}\text { LIQUID EFFLUENTS } & \\ \text { NUCLIDES RELEASED } & \text { ACTIVITY } \\ \text { SB-1 } 124 & <1.91 E-02 \\ \text { I-131 } & <7.86 E-02 \\ \text { XE-133 } & <1.31 E-01 \\ \text { CS-134 } & <3.34 E-01 \\ \text { XE-13,5 } & <1.13 E-02 \\ \text { CS-136 } & <2.40 E-03 \\ \text { CS-137 } & <4.40 E-01 \\ \text { BA-LA-140 } & <2.18 E-02 \\ \text { CE-141 } & <1.56 E-02 \\ \text { UNIDENTIFIED } & <9.14 E-02 \\ & \\ \text { TRITIUM } & (\mathrm{CI}) \\ \text { TOTAL AIRBORNE RELEASE } & 4.54 E+00 \\ \text { TOTAL LIQUID RELEASE } & <8.37 E+00\end{array}$

VOLUME OF LIQUID WASTE RELEASED (PRIOR TO DILUTION) LITERS 1.12E+07 VOLUME OF DILUTION WATER USED DURING PERIOD
LITERS $5,16 E+10$

N/A=NOT APPLICABLE

N/D=NOT DETECTED

$N / R=$ NOT REPORTED 
INSTALLATION : COOPER

EFFLUENT AND WASTE DISPOSAL ANNUAL REPORT FOR YEAR 1981 SOLID EFFLUENTS

SOLID WASTE DISPOSITION NUMBER OF SHIPMENTS MODE OF TRANSPORTATION 71 SOLE USE VEHICLE SOLE USE VEHICLE

DESTINATION BEATTY, NV

RICHLAND, WA

IRRADIATED FUEL SHI PMENTS (DISPOS ITION)

NUMBER OF SHIPMENTS MODE OF TRANSPORTATION

DESTINATION

O

ESTIMATE OF MAJOR NUCLIDE COMPOS ITION(BY TYPE OF WASTE)

$A$

AG-110M

BA-LA-140

CO-58

CO-60

CR-51

CS-134

CS-137

FE-59

I-13|

MN-54

$\mathrm{ZN}-65$

ZR-NB-95

B

AG- $110 \mathrm{M}$

CO-58

CO-60

CR-51

CS-134

CS-137

FE-59

I-13I

MN -34

$\mathrm{ZN}-63$

ZR-NB-95

\begin{tabular}{|c|c|c|}
\hline & JAN-JUNE & JULY-DEC \\
\hline$\%$ & $2.09 E+00$ & $2.94 E+00$ \\
\hline$\%$ & & 1. $52 \mathrm{E}-01$ \\
\hline$\%$ & $3.35 E+00$ & $2.50 \mathrm{E}+00$ \\
\hline$\%$ & $2.49 \mathrm{E}+01$ & 3. $89 E+01$ \\
\hline$\%$ & $2.43 E+01$ & $4.37 E+00$ \\
\hline$\%$ & $1.23 E+01$ & $1.535+01$ \\
\hline$\%$ & $1.71 E+01$ & $2.03 E+01$ \\
\hline$\%$ & $4.49 \mathrm{E}-01$ & $1.93 E-01$ \\
\hline$\%$ & $1.69 \mathrm{E}-01$ & $1.25 E-01$ \\
\hline ж & $1.37 E+01$ & $1.36 \mathrm{E}+01$ \\
\hline$\%$ & $1.06 \mathrm{E}+00$ & $1.28 \mathrm{E}+00$ \\
\hline$\%$ & $4.29 E-01$ & $2.50 \mathrm{E}-01$ \\
\hline$\%$ & $1.60 \mathrm{E}+00$ & $2.14 E+00$ \\
\hline$\%$ & $1.43 E+00$ & $2.18 E+00$ \\
\hline$\%$ & $2.57 E+01$ & $2.55 E+01$ \\
\hline$\%$ & 2. $29 E+01$ & $2.42 E+01$ \\
\hline$\%$ & $1.46 E+01$ & $1.35 E+01$ \\
\hline x & $2.03 \mathrm{E}+01$ & $1.88 E+01$ \\
\hline 8 & & $2.07 E-01$ \\
\hline$\%$ & $6.31 \mathrm{E}-01$ & $1.30 \mathrm{E}-01$ \\
\hline$\%$ & $1.14 E+01$ & $1.23 E+01$ \\
\hline 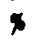 & $9.57 \mathrm{E}-01$ & $9.79 \mathrm{E}-01$ \\
\hline$\%$ & $1.43 \mathrm{E}-01$ & $2.72 \mathrm{E}-01$ \\
\hline
\end{tabular}

TYPE OF WASTE

A. SPENT RESINS, FILTER SLUDGES, EVAPORATOR BOTTOMS, ETC.

B. DRY COMPRESSIBLE WASTE, CONTAMINATED EQUI PMENT, ETC.

1. 43E-01

2.72E-01

C. IRRADIATED COMPONENTS, CONTROL RODS, ETC

D. OTHER

UNIT YEAR TOTAL

M3 $1.51 \mathrm{E}+02$

CI $4.40 E+02$

M3 $3.48 \mathrm{E}+02$

CI $2.82 E+00$

M3

CI

M3

CI

N/A=NOT APPLICABLE

N/D $=$ NOT DETECTED

N/R=NOT REPORTED 
EFFLUENT AND WASTE DISPOSAL ANNUAL REPORT FOR YEAR 1981 AIRBORNE AND LIQUID EFFLUENTS

UNIT NUMBER: 3

TYPE : PWR

DOCKET NO . : 50-302

COOLING WATER SOURCE: GULF OF MEXICO

\begin{tabular}{|c|c|}
\hline \multicolumn{2}{|l|}{ A IRBORNE EFFLUENTS } \\
\hline NUCLIDES RELEASED & ACTIVITY (CI) \\
\hline$A R-41$ & $<3.23 E+02$ \\
\hline CR-5I & $<6.97 \mathrm{E}-04$ \\
\hline MN -54 & $<7.01 \mathrm{E}-05$ \\
\hline $\mathrm{CO}-57$ & $<3.51 \mathrm{E}-05$ \\
\hline C0-58 & $<1.05 \mathrm{E}-04$ \\
\hline FE-59 & $<1.74 \mathrm{E}-04$ \\
\hline $\mathrm{CO}-6 \mathrm{Q}$ & $<1.03 \mathrm{E}-04$ \\
\hline $\mathrm{ZN}-65$ & $<2.17 \mathrm{E}-04$ \\
\hline KR-85 & $2.03 \mathrm{E}+03$ \\
\hline$K R-85 M$ & $1.40 \mathrm{E}+02$ \\
\hline $\mathbf{K R}-87$ & $<3.84 \mathrm{E}+01$ \\
\hline$K R-88$ & $<1.38 \mathrm{E}+02$ \\
\hline$K R-89$ & $<1.34 E+05$ \\
\hline SR-89 & $1.08 E-09$ \\
\hline SR-90 & $<1.71 \mathrm{E}-04$ \\
\hline NB-95 & $<7.05 E-05$ \\
\hline ZR-95 & $<1.19 \mathrm{E}-04$ \\
\hline AG-110M & $<1.00 \mathrm{E}-04$ \\
\hline SB-126 & $<7.10 \mathrm{E}-05$ \\
\hline $1-131$ & $<9.04 \mathrm{E}-03$ \\
\hline$X E-131 M$ & 1. $71 E+02$ \\
\hline $1-133$ & $<2.79 E-03$ \\
\hline$X E-133$ & $3.36 \mathrm{E}+04$ \\
\hline $\mathrm{XE}-133 \mathrm{M}$ & $6.42 E+02$ \\
\hline CS-134 & $<1.47 \mathrm{E}-03$ \\
\hline I-195 & $<1.47 \mathrm{E}+00$ \\
\hline$X E-135$ & $2.29 \mathrm{E}+09$ \\
\hline$X E-135 M$ & $<2.18 \mathrm{E}+02$ \\
\hline $\operatorname{cs}-137$ & $<1.96 \mathrm{E}-03$ \\
\hline $\mathrm{XE}-137$ & $<2.58 \mathrm{E}+04$ \\
\hline $\mathrm{XE}-138$ & $<7.16 \mathrm{E}+02$ \\
\hline BA-LA-140 & $<1.86 \mathrm{E}-04$ \\
\hline CE-141 & $<9.12 \mathrm{E}-05$ \\
\hline \multicolumn{2}{|l|}{ LIQUID EFFLUENTS } \\
\hline NUCLIDES RELEASED & ACTIVITY (CI) \\
\hline RB & $1.47 \mathrm{E}-07$ \\
\hline NA-24 & $<2.85 \mathrm{E}-03$ \\
\hline CR-51 & $<2.62 \mathrm{E}-02$ \\
\hline $\mathbf{Y N}-54$ & $<3.35 E-03$ \\
\hline Co-57 & $9.65 E-06$ \\
\hline CO-58 & $4.79 \mathrm{E}-02$ \\
\hline FE-59 & $<7.73 E-03$ \\
\hline $\mathrm{CO}-60$ & $1.30 \mathrm{E}-02$ \\
\hline
\end{tabular}

LICENSEE : FLOR I DA POWER

LICENSED POWER (MWT) :2452.00

INITIAL CRITICALITY: $01 / 14 / 77$

N/A=NOT APPLICABLE

N/D $=$ NOT DETECTED

N/R=NOT REPORTED 
EFFLUENT AND WASTE DISPOSAL ANNUAL REPORT FOR YEAR 1981 AIRBORNE AND LIQUID EFFLUENTS

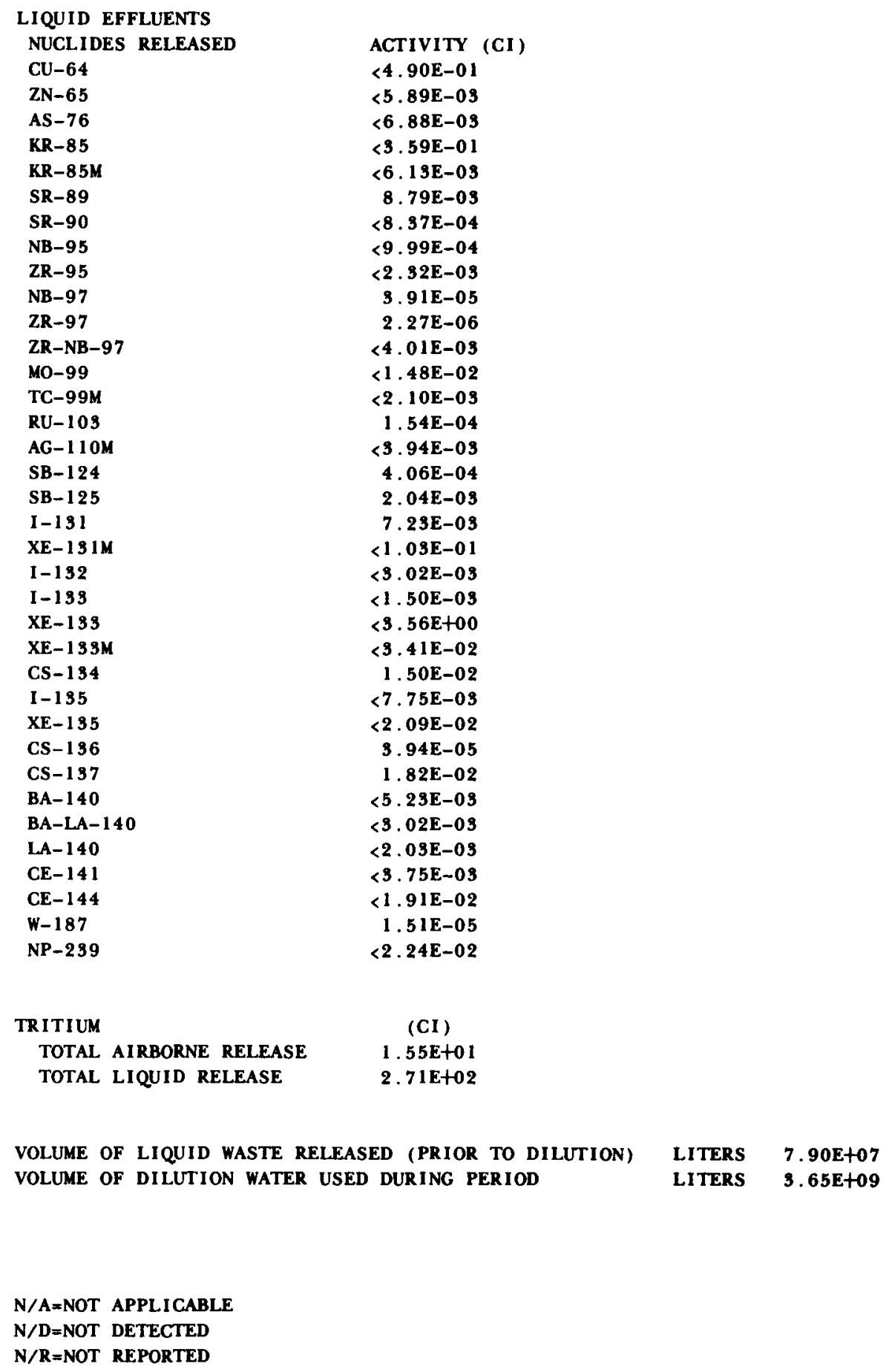

VOLUME OF LIQUID WASTE RELEASED (PRIOR TO DILUTION) VOLUME OF DILUTION WATER USED DURING PERIOD

N/A =NOT APPLICABLE

N/D=NOT DETECTED

$N / R=$ NOT REPORTED 
INSTALLATION : CRYSTAL RIVER

EFFLUENT AND WASTE DISPOSAL ANNUAL REPORT FOR YEAR 1981 SOLID EFFLUENTS

SOLID WASTE DISPOSITION NUMBER OF SHI PMENTS 40 41 MODE OF TRANSPORTATION EXCLUS IVE USE VEHICL TRUCK

IRRADIATED FUEL SHI PMENTS (DI SPOS ITION) NUMBER OF SHIPMENTS MODE OF TRANSPORTATION $\mathbf{0}$

ESTIMATE OF MAJOR NUCLIDE COMPOSITION(BY TYPE OF WASTE) A CO-58 $\mathrm{CO}-60$

Cs-134

CS-137

1-131

B

Co-58

$\mathrm{CO}-60$

CR-5 1

CS-134

CS-137

I-191

I-133
DESTINATION

BARNWELL, S.C.

BARNWELL, S.C.

DESTINATION

JAN-JUNE JULY-DEC
3. $00 \mathrm{E}+00$
4. $69 \mathrm{E}+00$
2. 30E+00
5. 10E+00
2.70E+01 2.63E+01
$5.91 E+01 \quad 5.34 E+01$
$6.50 \mathrm{E}+00$
7. 20E $+00 \quad 1.50 \mathrm{E}+01$
$1.67 \mathrm{E}+01 \quad 3.66 \mathrm{E}+00$
1. $39 E+00$
$1.02 \mathrm{E}+01 \quad 2.94 \mathrm{E}+00$
1.74E+01 4.52E+01
$4.04 \mathrm{E}+01 \quad 1.94 \mathrm{E}+00$

2. $40 E+00$

UNIT YEAR TOTAL

TYPE OF WASTE

A. SPENT RESINS, FILTER SLUDGES, EVAPORATOR

M3 $1.01 \mathrm{E}+03$

BOTTOMS, ETC.

B. DRY COMPRESSIBLE WASTE, CONTAMINATED

EQU I PMENT, ETC.

C. IRRADIATED COMPONENTS, CONTROL RODS, ETC.

CI $1.30 E+03$

M3 2.55E+02

CI $\quad 7.76 \mathrm{E}+01$

MS

CI

M3

CI 
EFFLUENT AND WASTE DISPOSAL ANNUAL REPORT FOR YEAR 1981 AIRBORNE AND LIQUID EFFLUENTS

UNIT NUMBER: 1

TYPE : PWR

DOCKET NO . : 50-346

COOLING WATER SOURCE: LAKE ERIE

AIRBORNE EFFLUENTS

NUCLIDES RELEASED

H-3

$\mathrm{KR}-85$

KR-85M

SR-89

I-13I

$\mathrm{XE}-131 \mathrm{M}$

$1-133$

$\mathrm{XE}-133$

$\mathrm{XE}-133 \mathrm{M}$

$X E-135$

$\mathrm{XE}-135 \mathrm{M}$

CS-137

LIQUID EFFLUENTS

NUCLIDES RELEASED

CR-5 I

MN -54

CO-58

FE-59

C0-60

SR-89

$\mathrm{Y}-91 \mathrm{M}$

NB-95

NB-97

ZR-97

MO-99

TC-99M

AG- $110 \mathrm{M}$

I-131

I-133

CS-134

CS-136

CS-137

BA-139

CE-141

TR ITIUM

TOTAL AIRBORNE RELEASE

TOTAL LIQUID RELEASE
ACTIVITY (CI)

8. $66 \mathrm{E}+00$

$7.04 \mathrm{E}+00$

1. $56 \mathrm{E}+00$

$6.83 \mathrm{E}-05$

5. 38E-02

3. $78 \mathrm{E}+00$

$6.04 \mathrm{E}-03$

9.83E+02

1. $51 \mathrm{E}+00$

1. $60 \mathrm{E}+01$

1. $00 \mathrm{E}+00$

2.97E-13

ACTIVITY (CI)

1. $49 \mathrm{E}-03$

3. 68E-03

3. 37E-01

1. 59E-04

1. $49 \mathrm{E}-02$

5. 28E-04

3. $51 \mathrm{E}-05$

1. 81 E-04

3. 92E-04

5. 40E-05

4. 63E-04

4. 82E-04

1. 26E-03

2. 43E-01

1. 09E-03

5. 95E-02

4. 89E-04

1. 27E-01

3. 27E-05

$1.21 E-05$

(CI)

8. 65E+00

1. $57 E+02$
LICENSEE : TOLEDO EDISON AND CLEVELAND ELECTRIC ILLUMINATING LICENSED POWER (MWT) : 2772.00

INITIAL CRITICALITY: $08 / 12 / 77$

VOLUME OF LIQUID WASTE RELEASED (PRIOR TO DILUTION) LITERS 1.26E+08 VOLUME OF DILUTION WATER USED DURING PERIOD

LITERS $2.77 \mathrm{E}+10$

N/A $=$ NOT APPLICABLE

$N / D=N O T$ DETECTED

N/R=NOT REPORTED 
EFFLUENT AND WASTE DISPOSAL ANNUAL REPORT FOR YEAR 1981 SOLID EFFLUENTS

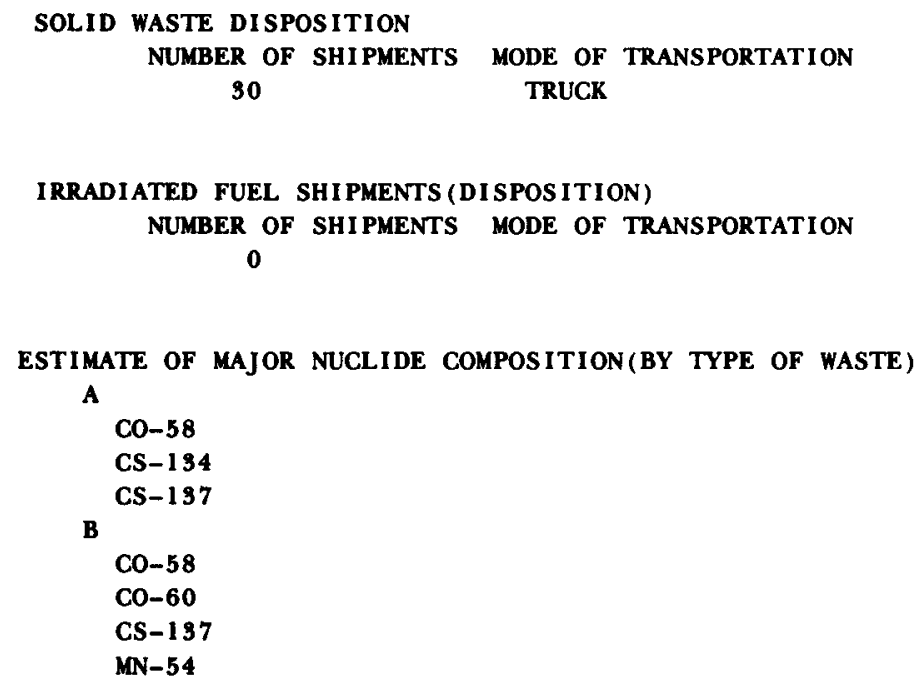

$\begin{array}{ccc} & \text { JAN-JUNE } & \text { JULY-DEC } \\ & & \\ \% & 1.65 E+01 & 6.71 E+00 \\ \% & 1.92 E+01 & 2.58 E+01 \\ \% & 5.04 E+01 & 5.89 E+01 \\ & & \\ \% & 6.22 E+01 & 4.44 E+01 \\ \% & & 3.69 E+01 \\ \% & 1.53 E+01 & \\ \% & 1.04 E+01 & 9.07 E+00\end{array}$

TYPE OF WASTE

A. SPENT RESINS, FILTER SLUDGES, EVAPORATOR BOTTOMS, ETC.

B. DRY COMPRESSIBLE WASTE, CONTAMINATED EQUI PMENT, ETC.

$1.04 \mathrm{E}+01$

$9.07 E+00$

C. IRRADIATED COMPONENTS, CONTROL RODS, ETC.

D. OTHER 
EFFLUENT AND WASTE DISPOSAL ANNUAL REPORT FOR YEAR 1981 AIRBORNE AND LIQUID EFFLUENTS

UNIT NUMBER: 1

TYPE : $\quad$ BWR

DOCKET NO. : 50-10

COOLING WATER SOURCE: RANKAKEE RIVER

\section{AIRBORNE EFFLUENTS}

NUCLIDES RELEASED

MN-54

C0-58

FE-59

C0-60

ZN-65

SR-89

SR-90

NB-95

ZR-95

AG- $110 \mathrm{M}$

I-131

CS-134

CS -137

BA-140

CE- 141

CE-144

TRITIUM

TOTAL AIRBORNE RELEASE
ACTIVITY (CI)

8. 55E-04

1. $55 \mathrm{E}-06$

4. $31 \mathrm{E}-07$

2.82E-03

4. 52E-07

9. 40E-04

6. 20E-05

8.41E-04

4.88E-06

2.87E-07

2.61E-03

3. 28E-05

1.75E-03

1. 29E-06

5. 32E-06

2.87E-06

(CI)
LICENSEE : COMMON WEALTH EDISON LICENSED POWER (MWT) : $\mathbf{7 0 0 . 0 0}$

INITIAL CRITICALITY: 10/15/59

$N / A=N O T$ APPLICABLE

N/D=NOT DETECTED

N/R=NOT REPORTED 
EFFLUENT AND WASTE DISPOSAL ANNUAL REPORT FOR YEAR 1981 AIRBORNE AND LIQUID EFFLUENTS

UNIT NUMBER: 2

TYPE : BWR

DOCKET NO. : 50-237

COOLING WATER SOURCE: KANKAKEE RIVER

UNIT NUMBER: 3

TYPE : $\quad$ BWR

DOCKET NO. : 50-249

COOLING WATER SOURCE:KANKAKEE RIVER

\section{AIRBORNE EFFLUENTS}

NUCLIDES RELEASED

CR-51

MN-54

CO-58

FE-59

CO-60

$\mathrm{ZN}-65$

KR-85M

KR-87

KR-88

SR-89

SR-90

NB-95

ZR-95

RU-103

AG- $110 M$

SB-1 24

I-13|

I-133

$X E-133$

XE-133M

CS- 134

1-135

XE-135

XE-135M

CS-136

CS-137

XE- 138

BA-140

CE-141

CE-144

LIQUID EFFLUENTS

NUCLIDES RELEASED

MN-54

CO-58

CO-60

SR-89

SR-90

ZR-95

|-131

N/A=NOT APPLICABLE

N/D=NOT DETECTED

N/R=NOT REPORTED
LICENSEE : COMONWEALTH EDI SON LICENSED POWER (MWT) : 2527.00 INITIAL GRITICALITY : 06/09/72

LICENSEE : CONONWEALTH EDISON LICENSED POWER (MWT) $: 2527.00$ INITIAL CRITICALITY : $11 / 16 / 71$
ACTIVITY (CI)

1.26E-01

3. $57 \mathrm{E}-02$

$1.01 \mathrm{E}-02$

5. $11 \mathrm{E}-03$

3. 03E-01

3. 95E-03

7. 12E+02

1. $05 \mathrm{E}+03$

1. $75 \mathrm{E}+03$

1 . O3E+00

$5.81 \mathrm{E}-03$

2. 17E-02

1. $01 \mathrm{E}-02$

1. $67 \mathrm{E}-02$

5. 20E-05

3. 12E-04

4. $62 \mathrm{E}+00$

1. $53 \mathrm{E}+01$

3. $26 \mathrm{E}+03$

7. $13 \mathrm{E}+01$

1. 02E-02

2.53E+01

9. $11 \mathrm{E}+03$

$5.48 \mathrm{E}+03$

7. 03E-03

4. 23E-02

$1.81 \mathrm{E}+04$

4. $45 E+00$

1.36E-01

3. 82E-03
ACTIVIFY (CI)

3.89E-03

9.75E-04

2. 84E-02

1. $10 \mathrm{E}-03$

1. 66E-04

2.56E-04

1. 53E-02 
EFFLUENT AND WASTE DISPOSAL ANNUAL REPORT FOR YEAR 1981 AIRBORNE AND LIQUID EFFLUENTS

$\begin{array}{lc}\text { LIQUID EFFLUENTS } & \\ \text { NUCLIDES RELEASED } & \text { ACTIVITY (CIT } \\ \text { CS-134 } & 1.86 E-03 \\ \text { CS-137 } & 8.30 E-03 \\ \text { CE-14I } & 9.76 E-04 \\ & \\ & \\ \text { TRITIUM } & \text { (CI) } \\ \text { TOTAL AIRBORNE RELEASE } & 3.16 E+02 \\ \text { TOTAL LIQUID RELEASE } & 6.05 E+00\end{array}$

VOLUME OF LIQUID WASTE RELEASED (PRIOR TO DILUTION) VOLUME OF DILUTION WATER USED DURING PERIOD $\begin{array}{ll}\text { LITERS } & 4.43 \mathrm{E}+06 \\ \text { LITERS } & 1.63 \mathrm{E}+12\end{array}$

N/A=NOT APPLICABLE

N/D $=$ NOT DETECTED

N/R=NOT REPORTED 
INSTALLATION : DRESDEN $1,2,83$

EFFLUENT AND WASTE DISPOSAL ANNUAL REPORT FOR YEAR 1981 SOLID EFFLUENTS

SOLID WASTE DISPOSITION NUMBER, OF SHI PMENTS

197

MODE OF TRANSPOR'TATION

33

TRUCK

TRUCK

DESTINATION

BARNWELL, S.C.

RICHLAND, WA

TYPE OF WASTE

A. SPENT RESINS, FILTER SLUDGES, EVAPORATOR

UNIT YEAR TOTAL

BOTTOMS, ETC.

B . DRY COMPRESS IBLE WASTE, CONTAMINATED

$4.19 \mathrm{E}+02$

EQUI PMENT, ETC

CI 4.49E+03

M3 $7.19 \mathrm{E}+02$

C. IRRADIATED COMPONENTS, CONTROL

$9.69 \mathrm{E}+01$ RODS , ETC.

MS

D. OTHER 
EFFLUENT AND WASTE DISPOSAL ANNUAL REPORT FOR YEAR 1981 AIRBORNE AND LIQUID EFFLUENTS

UNIT NUMBER : 1

TYPE : $\quad$ BWR

DOCKET NO. : 50-331
LICENSEE : IOWA ELECTRIC LIGHT \& POWER

LICENSED POWER (MWT) : 1658.10

INITIAL CRITICALITY: 03/23/74

AIRBORNE EFFLUENTS

NUCLIDES RELEASED

$\mathrm{N}-13$

AR-4 1

CR-5 1

MN-54

CO-57

C0-58

FE-59

CO-60

KR-85

KR-85M

KR-87

KR-88

SR-89

SR-90

I- 131

I-133

XE- 133

CS-134

I-135

XE-135

$\mathrm{XE}-135 \mathrm{M}$

CS-197

CS- 138

$\mathrm{XE}-138$

BA-140

CE-141

UNI DENT I F I ED

TOTAL LIQUID RELEASE

ACTIVITY (CI)
$4.64 E+00$
$1.10 E+00$
$1.14 E-02$
$8.66 E-04$
$4.00 E-06$
$2.16 E-05$
$2.17 E-05$
$2.12 E-03$
$<4.44 E-01$
$3.06 E+01$
$4.76 E+00$
$2.77 E+01$
$3.36 E-04$
$3.52 E-05$
$1.42 E-02$
$1.64 E-01$
$1.61 E+02$
$1.19 E-05$
$1.49 E-01$
$5.55 E+01$
$<1.14 E+02$
$1.10 E-05$
$3.52 E-04$
$8.79 E+01$
$1.11 E-03$
$2.76 E-04$
$1.76 E-03$
N/A

VOLUME OF LIQUID WASTE RELEASED (PRIOR TO DILUTION) LITERS N/A VOLUME OF DILUTION WATER USED DURING PERIOD

$N / A=$ NOT APPLICABLE

$N / D=$ NOT DETECTED

N/R=NOT REPORTED 
INSTALLATION : DUANE ARNOLD

EFFLUENT AND WASTE DISPOSAL ANNUAL REPORT FOR YEAR 1981 SOLID EFFLUENTS

SOLID WASTE DISPOSITION

NUMBER OF SHIPMENTS MODE OF TRANSPORTATION 114

4

DESTINATION

BARNWELL, S.C.

BEATTY, NV

IRRADIATED FUEL SHI PMENTS (DISPOS ITION)

NUMBER OF SHIPMENTS MODE OF TRANSPORTATION

0

DESTINATION

TYPE OF WASTE

A. SPENT RESINS, FILTER SLUDGES, EVAPORATOR BOTTOMS, ETC.

B. DRY COMPRESSIBLE WASTE, CONTAMINATED EQUI PMENT, ETC.

C. IRRADIATED COMPONENTS, CONTROL RODS, ETC.

D. OTHER

UNIT YEAR TOTAL

M3 2.10E+02

CI $\quad 1.04 \mathrm{E}+09$

M3 4.87E+02

CI $2.58 E+01$

M3

CI

M3

CI

N/A=NOT APPLICABLE

$N / D=N O T$ DETECTED

N/R=NOT REPORTED 
EFFLUENT AND WASTE DISPOSAL ANNUAL REPORT FOR YEAR 1981 AIRBORNE AND LIQUID EFFLUENTS

UNIT NUMBER: 1

TYPE: PWR

DOCKET NO . : 50-348

COOLING WATER SOURCE :
LICENSEE : ALABAMA POWER

LICENSED POWER (MWT) : 2652.00

INITIAL CRITICALITY : 08/09/77

\begin{tabular}{|c|c|}
\hline \multicolumn{2}{|l|}{ AIRBORNE EFFLUENTS } \\
\hline NUCLI DES RELEASED & ACTIVITY (CI) \\
\hline AR-41 & $6.17 \mathrm{E}+01$ \\
\hline CR-51 & 1. $19 \mathrm{E}-05$ \\
\hline MN-54 & $6.40 \mathrm{E}-06$ \\
\hline $\mathrm{CO}-58$ & $5.10 E-01$ \\
\hline FE-59 & $0.00 E+00$ \\
\hline $\mathrm{CO}-60$ & $1.74 \mathrm{E}-04$ \\
\hline $\mathrm{ZN}-65$ & 3.52E-05 \\
\hline $\mathbf{K R}-\mathbf{8 5}$ & $7.08 E+01$ \\
\hline$K R-85 M$ & 1. $70 \mathrm{E}+00$ \\
\hline $\mathbf{K R}-\mathbf{8 7}$ & $4.98 \mathrm{E}-03$ \\
\hline KR-88 & $1.53 \mathrm{E}-02$ \\
\hline $\mathbf{K R}-\mathbf{8 9}$ & $0.00 E+00$ \\
\hline SR-89 & 3. 57E-04 \\
\hline $\mathbf{K R}-\mathbf{9 0}$ & $2.72 \mathrm{E}-07$ \\
\hline SR-90 & $8.97 E-11$ \\
\hline ZR-95 & $7.21 \mathrm{E}-06$ \\
\hline SB-1 24 & $0.00 E+00$ \\
\hline I-1 131 & $3.00 \mathrm{E}-03$ \\
\hline$X E-131 M$ & 8.53E-01 \\
\hline$I-139$ & 1. $39 \mathrm{E}-02$ \\
\hline$X E-133$ & 8.00E +01 \\
\hline $\mathrm{XE}-139 \mathrm{M}$ & $3.21 \mathrm{E}-01$ \\
\hline CS-134 & $2.23 E-02$ \\
\hline$X E-135$ & 5. $28 E+00$ \\
\hline$X E-135 M$ & $2.51 \mathrm{E}-11$ \\
\hline CS-136 & $0.00 E+00$ \\
\hline CS-137 & $8,74 E-02$ \\
\hline$X E-137$ & $2.93 E-02$ \\
\hline $\mathrm{XE}-1 \mathbf{3 8}$ & $5.55 \mathrm{E}-03$ \\
\hline BA-140 & $5.39 E-06$ \\
\hline $\mathrm{CE}-141$ & 1.58E-06 \\
\hline \multicolumn{2}{|l|}{ LIQUID EFFLUENTS } \\
\hline NUCLIDES RELEASED & ACTIVITY (CI) \\
\hline $\mathrm{H}-\mathbf{3}$ & $1.65 \mathrm{E}+02$ \\
\hline NA-24 & 5.32E-06 \\
\hline CR-51 & $9.95 \mathrm{E}-04$ \\
\hline$M N-54$ & $9.38 E-04$ \\
\hline $\mathrm{CO}-57$ & 1. $52 \mathrm{E}-04$ \\
\hline Co-58 & $5.06 \mathrm{E}-03$ \\
\hline FE-59 & $2.49 \mathrm{E}-04$ \\
\hline CO-60 & $1.21 \mathrm{E}-02$ \\
\hline CU-64 & $7.33 E-03$ \\
\hline $\mathrm{ZN}-65$ & $1.78 \mathrm{E}-06$ \\
\hline
\end{tabular}

N/A=NOT APPLICABLE N/D $=$ NOT DETECTED N/R=NOT REPORTED 
EFFLUENT AND WASTE DISPOSAL ANNUAL REPORT FOR YEAR 1981 AIRBORNE AND LIQUID EFFLUENTS

$\begin{array}{lcl}\text { LIQUID EFFLUENTS } & \\ \text { NUCLIDES RELEASED } & \text { ACTIVITY (CI) } \\ \text { SR-89 } & 6.72 E-05 \\ \text { SR-90 } & 2.61 E-06 \\ \text { NB-95 } & 5.76 E-04 \\ \text { ZR-95 } & 2.65 E-05 \\ \text { RU-106 } & 2.22 E-04 \\ \text { AG-110M } & 2.04 E-03 \\ \text { I-131 } & 1.93 E-02 \\ \text { I-133 } & 4.83 E-03 \\ \text { XE-133 } & 3.48 E-02 \\ \text { CS-134 } & 2.32 E-03 \\ \text { I-135 } & 2.98 E-04 \\ \text { XE-135 } & 1.43 E-05 \\ \text { CS-136 } & 6.76 E-05 \\ \text { CS-137 } & 1.04 E+00 \\ \text { LA-140 } & 9.38 E-05 \\ & & \\ \text { TRITIUM } & \text { (CI) } \\ \text { TOTAL AIRBORNE RELEASE } & 6.80 E+01 \\ \text { TOTAL LIQUID RELEASE } & 1.65 E+02 & \\ & & \\ \text { VOLUME OF LIQUID WASTE RELEASED (PRIOR TO DILUTION) } & \text { LITERS } & \\ \text { VOLUME OF DILUTION WATER USED DURING PERIOD } & \text { LITERS } & 6.82 E+10\end{array}$

N/A =NOT APPLICABLE

$N / D=N O T$ DETECTED

$N / R=N O T$ REPORTED 
EFFLUENT AND WASTE DISPOSAL ANNUAL REPORT FOR YEAR 1981 AIRBORNE AND LIQUID EFFLUENTS

UNIT NUMBER : 2

TYPE: PWR

DOCKET NO . : 50-364

COOLING WATER SOURCE:
LICENSEE : ALABAMA POWER

LICENSED POWER (MWT) : 2652.00 INITIAL CRITICALITY:05/05/81

\begin{tabular}{|c|c|}
\hline \multicolumn{2}{|l|}{ AIRBORNE EFFLUENTS } \\
\hline NUCLIDES RELEASED & ACTIVITY (CI) \\
\hline$A R-41$ & $2.82 E-04$ \\
\hline CR-51 & $1.05 E-05$ \\
\hline MN-54 & $5.76 \mathrm{E}-08$ \\
\hline C0-58 & $1.21 E-05$ \\
\hline FE-59 & $4.11 \mathrm{E}-08$ \\
\hline $\mathrm{CO}-60$ & $1.13 \mathrm{E}-04$ \\
\hline $\mathrm{ZN}-65$ & $8.28 E-08$ \\
\hline$K R-85$ & $1.64 \mathrm{E}-02$ \\
\hline $\mathbf{K R}-85 \mathrm{M}$ & 1. $52 \mathrm{E}-03$ \\
\hline KR-87 & $5.28 E-08$ \\
\hline KR-88 & $7.09 \mathrm{E}-08$ \\
\hline KR-89 & $2.37 E-08$ \\
\hline SR-89 & $1.07 E-05$ \\
\hline KR-90 & 1.52E-12 \\
\hline ZR-95 & $6.80 E-08$ \\
\hline SB-124 & $1.53 \mathrm{E}-07$ \\
\hline$I-191$ & $2.67 E-05$ \\
\hline$X E-131 M$ & $5.49 E-03$ \\
\hline I-133 & $6.48 \mathrm{E}-03$ \\
\hline$X E-133$ & $2.51 E+00$ \\
\hline$X E-133 M$ & $6.75 E-02$ \\
\hline CS-134 & $4.16 E-06$ \\
\hline$X E-135$ & $2.49 E-03$ \\
\hline $\mathrm{XE}-135 \mathrm{M}$ & $2.84 E-08$ \\
\hline CS-136 & 3. 39E-08 \\
\hline CS-137 & $1.53 \mathrm{E}-07$ \\
\hline$X E-137$ & $1.79 \mathrm{E}-06$ \\
\hline$X E-138$ & 8.09E-08 \\
\hline BA- 140 & $6.79 \mathrm{E}-06$ \\
\hline CE-141 & $5.21 \mathrm{E}-08$ \\
\hline \multicolumn{2}{|l|}{ LIQUID EFFLUENTS } \\
\hline NUCLIDES RELEASED & ACTIVITY (CI) \\
\hline H-3 & 5. $78 \mathrm{E}+02$ \\
\hline NA-24 & $8.06 \mathrm{E}-03$ \\
\hline P-32 & $6.29 \mathrm{E}-04$ \\
\hline CR-5 1 & $2.43 E-04$ \\
\hline MN-54 & $4.04 \mathrm{E}-04$ \\
\hline FE-55 & $4.64 E-03$ \\
\hline $\mathrm{CO}-57$ & 3.59E-06 \\
\hline Co-58 & 3. 22E-03 \\
\hline FE-59 & $2.99 \mathrm{E}-04$ \\
\hline $\mathrm{CO}-60$ & $4.71 \mathrm{E}-03$ \\
\hline$C U-64$ & $6.17 E-05$ \\
\hline
\end{tabular}

N/A=NOT APPLICABLE $N / D=N O T$ DETECTED N/R=NOT REPORTED 
EFFLUENT AND WASTE DISPOSAL ANNUAL REPORT FOR YEAR 1981 AIRBORNE AND LIQUID EFFLUENTS

\section{LIQUID EFFLUENTS}

NUCLIDES RELEASED

$\mathrm{ZN}-65$

AS-76

SR-89

SR-90

NB-95

ZR-95

MO-99

TC-99M

RU-103

RU-106

AG-110M

I-131

I-132

I -133

$\mathrm{XE}-133$

CS -134

I-135

XE-135

CS-196

CS -137

BA-140

LA -140

CE-141

NP-239

\section{TRITIUM}

TOTAL AIRBORNE RELEASE

TOTAL LIQUID RELEASE
ACTIVITY (CI)

9.11E-07

2.41E-06

2.17E-05

9. $71 \mathrm{E}-07$

5. 35E-04

2.74E-05

8. 15E-06

2. 89E-05

6.02E-04

1. $02 \mathrm{E}-04$

2. 20E-03

5. 69E-04

1. 22E-05

5. 90E-05

4. 27E-02

8. 00E-05

9.66E-06

8. 84E-06

3. 52E-05

1. 10E-04

2.04E-05

6. 49E-05

4. 27E-07

1. 13E-04

(CI)

1. 25E+02

6. 34E+02

VOLUME OF LIQUID WASTE RELEASED (PRIOR TO DILUTION) LITERS 1.66E+08 VOLUME OF DILUTION WATER USED DURING PERIOD

LITERS $5.09 \mathrm{E}+10$

N/A=NOT APPLICABLE

N/D $=$ NOT DETECTED

$N / R=N O T$ REPORTED 
INSTALLATION : JOSEPH M. FARLEY $1 \& 2$

EFFLUENT AND WASTE DISPOSAL ANNUAL REPORT FOR YEAR 1981 SOLID EFFLUENTS

SOLID WASTE DISPOSITION NUMBER OF SHIPMENTS MODE OF TRANSPORTATION 38 TRUCK

DESTINATION

BARNWELL, S.C.

ESTIMATE OF MAJOR NUCLIDE COMPOSITION(BY TYPE OF WASTE)

A

C0-58

CO-60

CR-51

CS-134

CS-137

MN-54

B

C0-58

CO-60

CS-134

CS-197

TYPE OF WASTE

A. SPENT RESINS, FILTER SLUDGES, EVAPORATOR BOTTOMS, ETC.

B. DRY COMPRESS IBLE WASTE, CONTAMINATED EQUI PMENT, ETC.

C. IRRADIATED COMPONENTS, CONTROL RODS, ETC.

D. OTHER

JAN-JUNE JULY-DEC

$\begin{array}{lll}\% & 2.15 E+01 & 1.69 E+01 \\ \% & 1.63 E+01 & 7.21 E+01 \\ \% & 3.36 E+00 & 5.00 E-01 \\ \% & 3.42 E+01 & 2.20 E+00 \\ \% & 9.54 E+00 & 4.00 E-01 \\ \% & 7.89 E+00 & 7.90 E+00 \\ & & \\ \% & 4.75 E+01 & 7.00 E+00 \\ \% & 4.25 E+01 & 8.06 E+01 \\ \% & 5.40 E+00 & 1.34 E+00 \\ \% & 7.00 E+00 & 1.73 E-01\end{array}$

UNIT YEAR TOTAL

M3 $1.49 \mathrm{E}+02$

CI $\quad 7.04 E+02$

M3 4.15E+02

CI $1.56 \mathrm{E}+01$

M3 $0.00 E+00$

CI $\quad 0.00 E+00$

M3 O. OOE +00

CI $\quad 0.00 E+00$

N/A=NOT APPLICABLE

N/D=NOT DETECTED

N/R=NOT REPORTED 
EFFLUENT AND WASTE DISPOSAL ANNUAL REPORT FOR YEAR 1981

AIRBORNE AND LIQUID EFFLUENTS

UNIT NUMBER : 1

TYPE: BWR

DOCKET NO. : 50-383

COOLING WATER SOURCE : LAKE ONTARIO

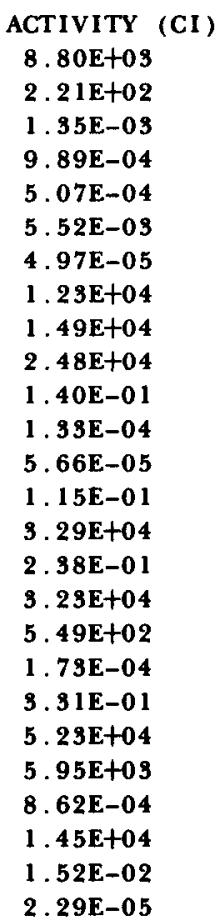

LICENSEE : POWER AUTHORITY OF THE STATE OF NEW YORK LICENSED POWER (MWT):2436.00 INITIAL CRITICALITY: $11 / 17 / 74$

$\begin{array}{lc}\begin{array}{l}\text { LIQUID EFFLUENTS } \\ \text { NUCLIDES RELEASED }\end{array} \\ \text { NA-24 } & \\ \text { CR-5 1 } & 3.77 E-02 \\ \text { MN-54 } & 4.79 E-02 \\ \text { CO-58 } & 3.44 E-01 \\ \text { FE-59 } & 2.08 E-01 \\ \text { CO-60 } & 1.10 E-02 \\ \text { ZN-65 } & 1.40 E+00 \\ \text { AS-76 } & 4.57 E-02 \\ \text { KR-85M } & 2.08 E-04 \\ \text { SR-90 } & 7.28 E-06 \\ \text { ZR-NB-95 } & 1.27 E-04 \\ \text { MO-99 } & 2.54 E-04 \\ \text { TC-99M } & 6.44 E-04 \\ \text { AG-110M } & 1.67 E-03 \\ \text { SB-124 } & 2.21 E-04 \\ \end{array}$

N/A=NOT APPLICABLE $N / D=N O T$ DETECTED N/R=NOT REPORTED 
EFFLUENT AND WASTE DISPOSAL ANNUAL REPORT FOR YEAR 1981 AIRBORNE AND LIQUID EFFLUENTS

$\begin{array}{lr}\text { LIQUID EFFLUENTS } & \\ \text { NUCLIDES RELEASED } & \text { ACTIVITY } \\ \text { I-191 } & 4.50 E-02 \\ \text { I-133 } & 5.35 E-03 \\ \text { XE-133 } & 3.98 E-02 \\ \text { CS-134 } & 8.00 E-02 \\ \text { I-195 } & 2.47 E-03 \\ \text { XE-135 } & 5.85 E-02 \\ \text { CS-137 } & 1.26 E-01 \\ \text { BA-LA-140 } & 1.12 E-03 \\ \text { CE-141 } & 6.99 E-05 \\ \text { CE-144 } & 6.58 E-04 \\ \text { NP-239 } & 4.29 E-02 \\ & \\ \text { TRITIUM } & (C I) \\ \text { TOTAL AIRBORNE RELEASE } & 6.65 E+00 \\ \text { TOTAL LIQUID RELEASE } & 4.11 E+00\end{array}$

VOLUME OF LIQUID WASTE RELEASED (PRIOR TO DILUTION) LITERS $1.81 \mathrm{E}+07$ VOLUME OF DILUTION WATER USED DURING PERIOD

LITERS 6.81E+11

N/AFNOT APPLICABLE

$N / D=N O T$ DETECTED

$N / R=$ NOT REPORTED 
INSTALLATION: JAMES A. FITZPATRICK

EFFLUENT AND WASTE DISPOSAL ANNUAL REPORT FOR YEAR 1981 SOLID EFFLUENTS

SOLID WASTE DISPOSITION NUMBER OF SHI PMENTS 86

16

MODE OF TRANSPORTATION

TRUCK

TRUCK

IRRADIATED FUEL SHIPMENTS (DISPOSITION)

NUMBER OF SHIPMENTS MODE OF TRANSPORTATION

0

ESTIMATE OF MAJOR NUCLIDE COMPOSITION(BY TYPE OF WASTE)

A

CO-58

Co-60

CR-51

CS-134

CS-137

MN-54

$\mathrm{ZN}-65$
DESTINATION

BARNWELL, S.C.

RICHLAND, WA

DESTINATION

JAN-JUNE JULY-DEC
4. $00 \mathrm{E}+00$
3. 10E+00
6. $32 E+01$
3. $90 \mathrm{E}+00$
1. 70E+00
3. 30E+00
1. $20 \mathrm{E}+01$
3. 70E+00
7. $10 \mathrm{E}+00$
1. $02 \mathrm{E}+01$
3. $20 E+00$
3. 30E+00

UNIT YEAR TOTAL

M3 3.99E+02

CI $\quad 1.61 \mathrm{E}+03$

M3 4.62E+02

CI $2.00 \mathrm{E}+01$

M3

CI

M3

CI

N/A $=$ NOT APPLICABLE

N/D=NOT DETECTED

$N / R=N O T$ REPORTED 
EFFLUENT AND WASTE DISPOSAL ANNUAL REPORT FOR YEAR 1981 AIRBORNE AND LIQUID EFFLUENTS

UNIT NUMBER: 1

TYPE : PWR

DOCKET NO . : 50-285

COOLING WATER SOURCE:MISSOURI RIVER

AIRBORNE EFFLUENTS NUCLIDES RELEASED

AR-41

KR-85

KR-85M

KR-87

KR-88

SR-89

SR-90

I - 131

$\mathrm{XE}-131 \mathrm{M}$

I -133

$\mathrm{XE}-133$

$\mathrm{XE}-133 \mathrm{M}$

CS-1 134

I -135

$\mathrm{XE}-135$

XE-135M

CS-197

$\mathrm{XE}-138$

BA-140

LA-140

LIQUID EFFLUENTS

NUCLI DES RELEASED

CR-51

MN-54

CO-57

CO-58

FE-59

CO-60

$\mathrm{ZN}-65$

SR-89

SR-90

NB-95

ZR-95

MO-99

TE-99M

RU-103

SN-117M

SB-1 24

I -131

I-139

XE-133

CS-134

XE-195

N/A=NOT APPLICABLE N/D $=$ NOT DETECTED N/R=NOT REPORTED
LICENSEE : OMAHA PUBLIC POWER LICENSED POWER (MWT) : 1420.00 INITIAL CRITICALITY:08/06/73

$$
\begin{aligned}
& \text { ACTIVITY (CI) } \\
& 3.49 E+00 \\
& 6.15 E+01 \\
& 2.99 E-01 \\
& 3.61 E-01 \\
& 4.71 E-01 \\
& 4.43 E-01 \\
& 1.20 E-07 \\
& 3.53 E-03 \\
& 3.68 E+01 \\
& 3.46 E-06 \\
& 1.11 E+03 \\
& 5.33 E+00 \\
& 4.44 E-06 \\
& 1.65 E-04 \\
& 3.18 E+00 \\
& 4.41 E-01 \\
& \text { 3.88E-06 } \\
& 1.20 E+00 \\
& \text { N/D } \\
& \text { N/D }
\end{aligned}
$$

ACTIVITY (CI)

$2.10 \mathrm{E}-03$

$1.01 \mathrm{E}-03$

3. 38E-04

3. 02E-02

N/D

3.85E-03

N/D

6. 16E-05

3. $98 \mathrm{E}-06$

$5.41 \mathrm{E}-04$

2.95E-04

N/D

N/D

N/D

1.04E-04

3. $18 \mathrm{E}-04$

4. $98 \mathrm{E}-03$

$\mathrm{N} / \mathrm{D}$

3. $23 E+00$

2. $21 \mathrm{E}-02$

1. 72E-02 
EFFLUENT AND WASTE DISPOSAL ANNUAL REPORT FOR YEAR 1981 AIRBORNE AND LIQUID EFFLUENTS

$$
\begin{aligned}
& \text { CS-136 } \\
& \text { CS-137 } \\
& B A-140 \\
& \text { LA-140 } \\
& \text { CE-141 }
\end{aligned}
$$$$
\text { LIQUID EFFLUENTS }
$$$$
\text { NUCLIDES RELEASED }
$$

TRITIUM

TOTAL AIRBORNE RELEASE

TOTAL LIQUID RELEASE

\author{
ACTIVITY (CI) \\ 3. $72 \mathrm{E}-04$ \\ 4. 68E-02 \\ 1. 27E-03 \\ 6. 60E-05 \\ N/D
}

(CI)

8. 15E+01

$2.42 \mathrm{E}+02$

VOLUME OF LIQUID WASTE RELEASED (PRIOR TO DILUTION) LITERS 6.42E+07 VOLUME OF DILUTION WATER USED DURING PERIOD

LITERS

1. $12 \mathrm{E}+12$ 
INSTALLATION : FORT CALHOUN

EFFLUENT AND WASTE DISPOSAL ANNUAL REPORT FOR YEAR 1981 SOLID EFFLUENTS

SOLID WASTE DISPOSITION NUMBER OF SHI PMENTS

$\begin{array}{rr}11 & \text { TRUCK } \\ 8 & \text { TRUCK }\end{array}$

\section{DESTINATION}

BARNWELL, S.C.

RICHLAND, WA

IRRADIATED FUEL SHI PMENTS (DISPOS ITION)

NUMBER OF SHIPMENTS MODE OF TRANSPORTATION

$\mathbf{0}$

N/A

DESTINATION

N/A

ESTIMATE OF MAJOR NUCLIDE COMPOSITION(BY TYPE OF WASTE)

A

Co-58

CO-60

CS-134

CS-137

B

CO-58

CO-60

CS- 134

CS- 137

$\begin{array}{lll} & \text { JAN-JUNE } & \text { JULY-DEC } \\ * & & 1.40 E+01 \\ \% & & 1.00 E+00 \\ * & 3.40 E+01 & 3.00 E+01 \\ * & 6.60 E+01 & 5.50 E+01 \\ & & \\ * & & 1.40 E+01 \\ \% & & 1.00 E+00 \\ * & 3.40 E+01 & 3.00 E+01 \\ * & 6.60 E+01 & 5.50 E+01\end{array}$

TYPE OF WASTE

A. SPENT RESINS, FILTER SLUDGES, EVAPORATOR

UNIT YEAR TOTAL BOTTOMS, ETC.

B. DRY COMPRESS I BLE WASTE, CONTAMINATED

EQUI PMENT, "ETC".

C. IRRADIATED COMPONENTS, CONTROL RODS, ETC

D. OTHER

$\begin{array}{ll}\text { M3 } & 1.29 \mathrm{E}+02 \\ \text { CI } & 9.08 \mathrm{E}+01 \\ \text { M3 } & 1.24 \mathrm{E}+02 \\ \text { CI } & 1.07 \mathrm{E}+01 \\ \text { M3 } & 0.00 \mathrm{E}+00 \\ \text { CI } & 0.00 \mathrm{E}+00 \\ \text { M3 } & \\ \text { CI } & \end{array}$

N/A=NOT APPLICABLE

$N / D=N O T$ DETECTED

N/R=NOT REPORTED 
EFFLUENT AND WASTE DISPOSAL ANNUAL REPORT FOR YEAR 1981 AIRBORNE AND LIQUID EFFLUENTS

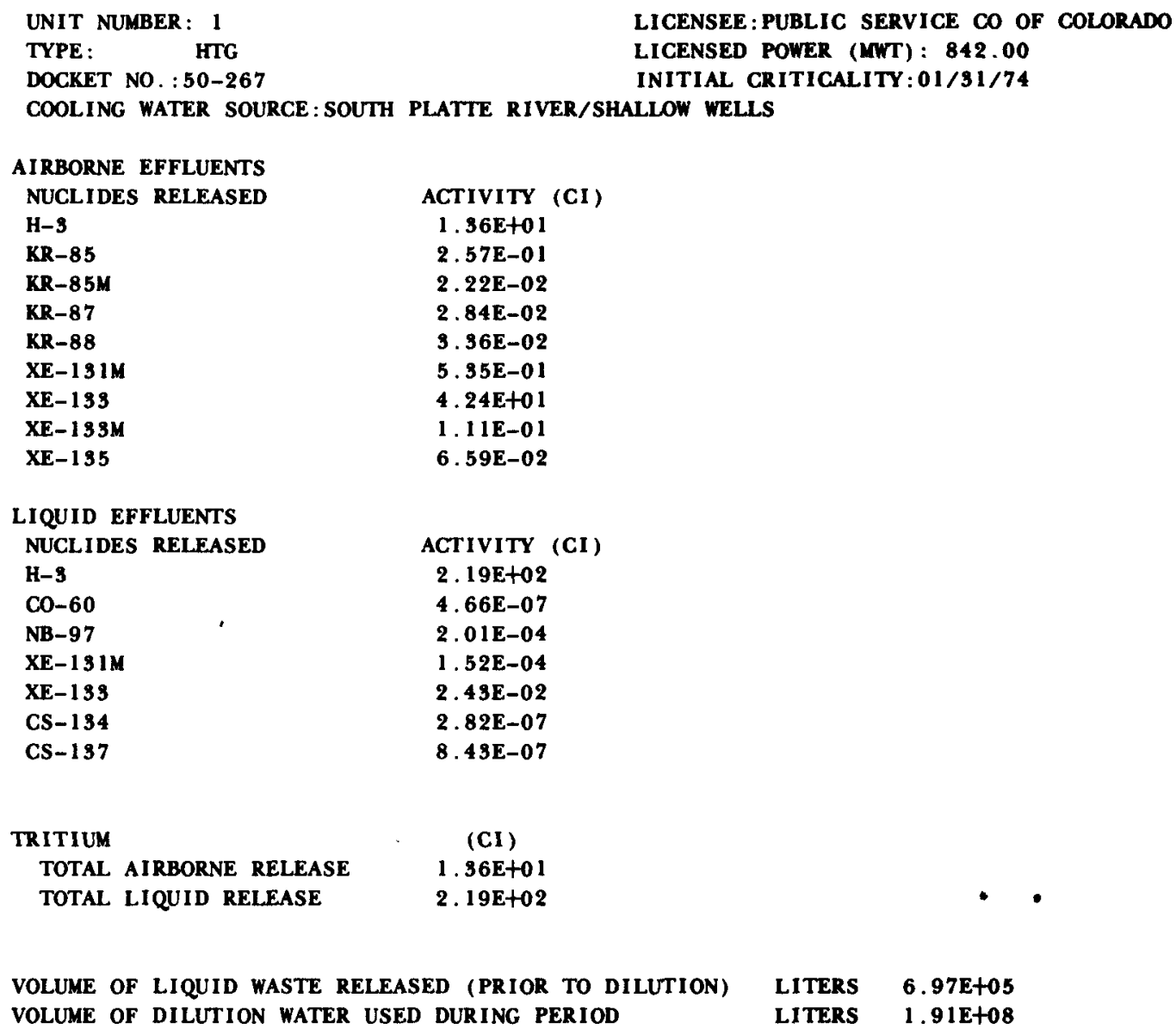

VOLUME OF LIQUID WASTE RELEASED (PRIOR TO DILUTION) LITERS 6.97E+05 VOLUME OF DILUTION WATER USED DURING PERIOD 
EFFLUENT AND WASTE DISPOSAL ANNUAL REPORT FOR YEAR 1981 AIRBORNE AND LIQUID EFFLUENTS

UNIT NUMBER : 1

TYPE : $\quad$ PWR

DOCKET NO. : 50-244

COOLING WATER SOURCE: LAKE ONTARIO

\section{AIRBORNE EFFLUENTS}

NUCLIDES RELEASED

AR-4 1

CR-51

C0-58

CO-60

KR-85

KR-85M

KR-87

KR-88

NB-95

ZR-95

RU-103

AG-110M

SB- 125

I-131

XE-131M

I-132

1-133

$X E-133$

$\mathrm{XE}-133 \mathrm{M}$

CS-134

I-135

XE- 135

XE-135M

CS-137

$\mathrm{XE}-138$

BA-LA- 140

\section{LIQUID EFFLUENTS}

NUCLIDES RELEASED

MN-54

CO-58

CO-60

I-131

CS-134

CS-137

BA-LA-140

CE-141

CE-PR-144

\section{TRITIUM}

TOTAL AIRBORNE RELEASE

TOTAL LIQUID RELEASE
LICENSEE : ROCHESTER GAS\&ELEC

LICENSED POWER (MWT) : 1520.10

INITIAL CRITICALITY: $11 / 08 / 69$
ACTIVITY (CI)

9.67E-01

2.04E-06

5.97E-07

3. $92 \mathrm{E}-06$

$1.30 \mathrm{E}+01$

5. 84E-01

7. 40E-02

9. 20E-02

5. 03E-08

1. 22E-07

1. 14E-07

8. 82E-08

2.57E-07

1. 12E-03

1. $79 \mathrm{E}+01$

3. 83E-03

$1.36 \mathrm{E}-03$

4. 83E+02

7. 22E-01

3. $63 \mathrm{E}-07$

1. 23E-04

$2.60 E+01$

$1.36 \mathrm{E}+00$

2. 75E-06

$1.21 \mathrm{E}+00$

6. 55E-07

ACTIVITY (CI)

1 . 07E-04

8. 18E-04

1. 38E-02

8. 88E-04

7. 18E-03

$1.41 E-02$

8. 83E-05

9.51E-05

1. $43 \mathrm{E}-03$

(CI)

7.01E+01

2. 40E+02

N/A=NOT APPLICABLE

$N / D=N O T$ DETECTED

N/R=NOT REPORTED 
EFFLUENT AND WASTE DISPOSAL ANNUAL REPORT FOR YEAR 1981

AIRBORNE AND LIQUID EFFLUENTS

VOLUME OF LIQUID WASTE RELEASED (PRIOR TO DILUTION)

VOLUME OF DILUTION WATER USED DURING PERIOD $\begin{array}{ll}\text { LITERS } & \text { 3.85E+07 } \\ \text { LITERS } & 6.97 \mathrm{E}+11\end{array}$

N/A=NOT APPLICABLE

$N / D=N O T$ DETECTED

N/R=NOT REPORTED 
INSTALLATION : R.E.GINNA

EFFLUENT AND WASTE DISPOSAL ANNUAL REPORT FOR YEAR 1981 SOLID EFFLUENTS

SOLID WASTE DISPOSITION

NUMBER OF SHIPMENTS MODE OF TRANSPORTATION

DESTINATION

15

5

BARNWELL SC

BEATTY NV

RICHLAND WA

TYPE OF WASTE

UNIT YEAR TOTAL

A. SPENT RESINS, FILTER SLUDGES, EVAPORATOR

M3 7.62E+01 BOTTOMS, ETC.

B . DRY COMPRESSIBLE WASTE, CONTAMINATED EQUI PMENT, ETC

CI $\quad 6.27 E+02$

M3 3.00E+02

CI $\quad 8.48 E+00$

C. IRRADIATED COMPONENTS, CONTROL RODS, ETC

M3 N/A

CI N/A

D. OTHER

M3

CI

N/A=NOT APPLICABLF

$N / D=N O T$ DETECTED

N/R $=$ NOT REPORTED 
EFFLUENT AND WASTE DISPOSAL ANNUAL REPORT FOR YEAR 1981 AIRBORNE AND LIQUID EFFLUENTS

\begin{tabular}{|c|c|c|c|}
\hline \multicolumn{4}{|l|}{ LIQUID EFFLUENTS } \\
\hline NUCLIDES RELEASED & ACTIVITY (CI) & & \\
\hline SR-90 & 8.01E-03 & & \\
\hline ZR-NB-95 & 3.06E-02 & & \\
\hline MO-99 & N/D & & \\
\hline TC-99M & N/D & & \\
\hline RU-103 & 1.26E-03 & & \\
\hline RU-106 & $6.06 \mathrm{E}-02$ & & \\
\hline$A G-110 M$ & $4.35 E-05$ & & \\
\hline SB-125 & $8.47 \mathrm{E}-03$ & & \\
\hline$I-131$ & $5.57 \mathrm{E}-02$ & & \\
\hline $1-132$ & $3.97 \mathrm{E}-02$ & & \\
\hline I - 193 & $1.58 \mathrm{E}-01$ & & \\
\hline XE-193 & $7.21 \mathrm{E}-01$ & & \\
\hline$X E-133 M$ & 1. $17 \mathrm{E}-02$ & & \\
\hline CS-134 & $2.78 \mathrm{E}-02$ & & \\
\hline$I-195$ & $4.16 \mathrm{E}-02$ & & \\
\hline XE-195 & $4.96 \mathrm{E}-02$ & & \\
\hline CS-137 & 8. $58 \mathrm{E}-02$ & & \\
\hline BA-LA- 140 & $2.94 \mathrm{E}-03$ & & \\
\hline$C E-141$ & $N / D$ & & \\
\hline $\mathrm{CE}-144$ & 9.87E-02 & & \\
\hline EU-1 54 & N/D & & \\
\hline TRITIUM & (CI) & & \\
\hline TOTAL AIRBORNE RELEASE & $8.65 E+01$ & & \\
\hline TOTAL LIQUID RELEASE & $5.29 E+03$ & & \\
\hline VOLUME OF LIQUID WASTE RE & ED (PRIOR TO DILUTION) & LITERS & $5.83 E+07$ \\
\hline VOLUME OF DILUTION WATER & DURING PERIOD & LITERS & $7.60 \mathrm{E}+11$ \\
\hline
\end{tabular}

N/A=NOT APPLICABLE

N/D=NOT DETECTED

N/R=NOT REPORTED 
INSTALLATION:HADDAM NECK

EFFLUENT AND WASTE DISPOSAL ANNUAL REPORT FOR YEAR 1981 SOLID EFFLUENTS

SOLID WASTE DISPOSITION NUMBER OF SHIPMENTS MODE OF TRANSPORTATION 48 TRUCK

DESTINATION

BARNWELL, S.C.

IRRADI ATED FUEL SHI PMENTS (DI SPOS ITION) NUMBER OF SHIPMENTS MODE OF TRANSPORTATION

DESTINATION

ESTIMATE OF MAJOR NUCLIDE COMPOSITION(BY TYPE OF WASTE)

A

CE-141

CE-144

CO-57

CO-58

CO-60

CR-51

CS-134

CS -137

MN-54

NB-95

RU- 103

RU-106

ZR-95

B

AG- $110 \mathrm{M}$

CE-141

CE-144

CO-57

CO-58

CO-60

CR-51

CS-134

CS-137

FE-59

I-131

UN-54

NA-22

NB-95

RU-1 03

RU-106

SB-1 25

ZR-95

D

AG-110M

CE-141

CE-144

CO-57

JAN-JUNE JULY-DEC
1. 29E+01
3.31E-01
2.71E+00
1. $44 E+00$
3. $70 \mathrm{E}+0$
3. 14E+0 1
$2.20 \mathrm{E}+01$
2.71E+01
1.52E-01
5. 38E-01
1. $42 \mathrm{E}-01$
$5.21 \mathrm{E}-01$
$4.81 \mathrm{E}+00$
3. $41 E+00$
5.82E-01
1. $82 \mathrm{E}+00$
2.74E+00
4. 19E+01 7.27E+00
3.23E-01 1.19E+00

3. 88E-01

$1.74 E-01 \quad 1.32 E-01$

1.73E-01

1. $69 \mathrm{E}+01 \quad 1.01 \mathrm{E}+01$

4. 28E-01 3.15E-01

$2.48 \mathrm{E}+00 \quad 1.05 \mathrm{E}+01$

2.75E+01 2.82E+01

1. 44E+0 I

5. 18E-02

1. 43E-01 1.94E-0 1

$1.55 \mathrm{E}-01$

$6.02 \mathrm{E}-02$

2.72E+00 2.80E+00

2.60E-01 1.68E-01

8.54E-01 1.21E+00

1.39E+00

4.72E+01 2.85E+01

7.64E-01 7.57E-01.

$4.86 E-01 \quad 7.47 E-01$

$1.74 E-01 \quad 1.32 E-01$

1.79E-01

$1.69 \mathrm{E}+01 \quad 1.01 \mathrm{E}+01$

$4.28 E-01$ 3.15E-01

N/A=NOT APPLICABLE

N/D $=$ NOT DETECTED

N/R=NOT REPORTED 
INSTALLATION : HADDAM NECK

EFFLUENT AND WASTE DISPOSAL ANNUAL REPORT FOR YEAR 1981 SOLID EFFLUENTS

ESTIMATE OF MAJOR NUCLIDE COMPOSITION(BY TYPE OF WASTE) CO-58 C0-60 CR-5 1

CS-134

CS- 137

FE-59

I-131

MN-54

NA-22

NB-95

RU-103

RU-106

SB-125

ZR-95

$\begin{array}{ccc} & \text { JAN-JUNE } & \text { JULY-DEC } \\ \% & 2.48 E+00 & 1.05 E+01 \\ \% & 2.75 E+01 & 2.82 E+01 \\ \% & & 1.44 E+01 \\ \% & & 5.18 E-02 \\ \% & 1.43 E-01 & 1.94 E-01 \\ \% & & 1.55 E-01 \\ \% & & 6.02 E-02 \\ \% & 2.72 E+00 & 2.80 E+00 \\ \% & 2.60 E-01 & 1.68 E-01 \\ \% & 8.54 E-01 & 1.21 E+00 \\ \% & & 1.39 E+00 \\ \% & 4.72 E+01 & 2.85 E+01 \\ \% & 7.64 E-01 & 7.57 E-01 \\ \% & 4.86 E-01 & 7.47 E-01\end{array}$

TYPE OF WASTE

A. SPENT RESINS, FILTER SLUDGES, EVAPORATOR

UNIT YEAR TOTAL BOTTOMS, ETC.

B. DRY COMPRESS IBLE WASTE, CONTAMINATED EQUI PMENT, ETC.

C. IRRADIATED COMPONENTS, CONTROL RODS, ETC.

D. OTHER

DRY WASTE IN DRUMS

$\begin{array}{ll}\text { M3 } & 1.40 E+02 \\ \text { CI } & 6.34 E+02 \\ \text { M3 } & 2.77 E+02 \\ \text { CI } & 1.17 E+01 \\ \text { M3 } & \text { N/A } \\ \text { CI } & \text { N/A } \\ \text { M3 } & 2.03 E+01 \\ \text { CI } & 1.56 E+01\end{array}$

N/A=NOT APPLICABLE

N/D $=$ NOT DETECTED

N/R $=$ NOT REPORTED 
EFFLUENT AND WASTE DISPOSAL ANNUAL REPORT FOR YEAR 1981 AIRBORNE AND LIQUID EFFLUENTS

UNIT NUNBER: 1

TYPE: $\quad$ BWR

DOCKET NO . : 50-321

COOLING WATER SOURCE : ALTAMAHA RIVER

\section{AIRBORNE EFFLUENTS}

NUCLIDES RELEASED

$\mathrm{N}-13$

AR-41

CR-5 1

MN-54

CO-58

FE-59

CO-60

KR-85

KR-85M

KR-87

KR-88

KR-89

SR-89

SR-90

NB-95

ZR-95

$\mathrm{SN}-113$

I-131

XE-131M

I -133

XE-133

XE-139M

CS-134

I - 135

$X E-135$

XE-135M

CS- 137

XE-137

$\mathrm{XE}-138$

BA- 140

LA- 140

CE- 141

CE-144

LIQUID EFFLUENTS

NUCLIDES RELEASED

F-18

NA-24

P-32

CR-51

MN-54

FE-55

MN-56

CO-58
LICENSEE : GEORG IA POWER

LICENSED POWER (MWT) : 2436.00

INITIAL CRITICALITY : 09/12/74

N/A=NOT APPLICABLE $N / D=N O T$ DETECTED N/R=NOT REPORTED

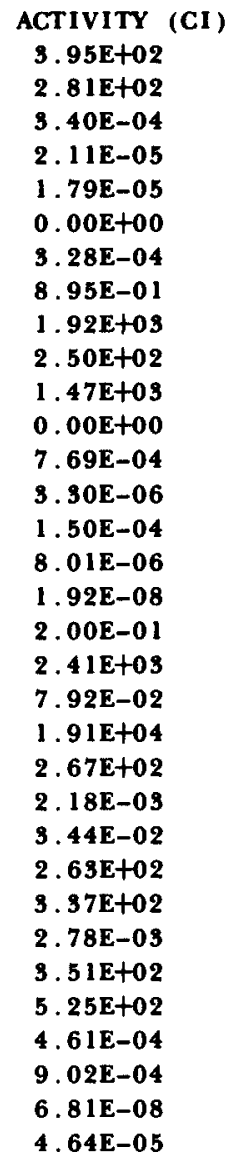


EFFLUENT AND WASTE DISPOSAL ANNUAL REPORT FOR YEAR 1981 AIRBORNE AND LIQUID EFFLUENTS

\begin{tabular}{|c|c|}
\hline LIQUID EFFLUENTS & \\
\hline NUCLIDES RELEASED & ACTIVITY (CI) \\
\hline FE-59 & $1.72 \mathrm{E}-04$ \\
\hline $\mathrm{CO-60}$ & $2.06 \mathrm{E}-02$ \\
\hline CU-64 & $7.37 \mathrm{E}-03$ \\
\hline NI -65 & $4.10 E-06$ \\
\hline$Z N-65$ & $4.07 E-02$ \\
\hline $\mathrm{ZN}-69 \mathrm{M}$ & $0.00 E+00$ \\
\hline AS-76 & $2.95 E-05$ \\
\hline KR-85M & $1.31 \mathrm{E}-05$ \\
\hline KR-87 & $0.00 E+00$ \\
\hline KR-88 & $2.63 \mathrm{E}-06$ \\
\hline RB-88 & $1.90 \mathrm{E}-05$ \\
\hline SR-89 & 1. $42 \mathrm{E}-03$ \\
\hline SR-90 & $0.00 E+00$ \\
\hline NB-95 & 1.07E-03 \\
\hline ZR-95 & $2.65 E-04$ \\
\hline NB-97 & $5.39 E-05$ \\
\hline ZR-97 & $9.50 E-06$ \\
\hline $30-99$ & $2.62 E-03$ \\
\hline TC-99M & 3. $70 \mathrm{E}-03$ \\
\hline$A G-110 M$ & $3.16 E-04$ \\
\hline $\mid-131$ & 4.82E-02 \\
\hline $\mathrm{XE}-131 \mathrm{M}$ & $1.79 \mathrm{E}-02$ \\
\hline$I-192$ & $0.00 E+00$ \\
\hline I-193 & 3.08E-03 \\
\hline$X E-133$ & 1. $10 E-01$ \\
\hline$X E-133 M$ & $2.08 E-03$ \\
\hline CS-134 & $7.36 \mathrm{E}-02$ \\
\hline$I-135$ & $2.77 E-04$ \\
\hline$X E-135$ & 8. 39E-02 \\
\hline$X E-135 M$ & 3.51E-06 \\
\hline CS-136 & $1.65 \mathrm{E}-03$ \\
\hline CS-137 & $8.97 E-02$ \\
\hline CS-138 & $1.00 \mathrm{E}-04$ \\
\hline$X E-138$ & $0.00 E+00$ \\
\hline$B A-140$ & $6.74 E-05$ \\
\hline$L A-140$ & $8.48 E-04$ \\
\hline$C E-141$ & $3.89 E-05$ \\
\hline CE-144 & 3. $44 \mathrm{E}-03$ \\
\hline$W-187$ & 1.33E-05 \\
\hline NP-239 & $6.86 E-04$ \\
\hline TRITIUM & (CI) \\
\hline TOTAL AIRBORNE RELEASE & 3. $52 \mathrm{E}+00$ \\
\hline TOTAL LIQUID RELEASE & $1.16 E+01$ \\
\hline
\end{tabular}

N/A=NOT APPLICABLE

N/D=NOT DETECTED

$N / R=$ NOT REPORTED 
EFFLUENT AND WASTE DISPOSAL ANNUAL REPORT FOR YEAR 1981 AIRBORNE AND LIQUID EFFLUENTS

VOLUME OF LIQUID WASTE RELEASED (PRIOR TO DILUTION) VOLUME OF DILUTION WATER USED DURING PERIOD
LITERS

LITERS
2. 86E+07

5. 73E+09

N/A=NOT APPLICABLE

N/D=NOT DETECTED

N/R=NOT REPORTED 
INSTALLATION: EDWIN I HATCH I

EFFLUENT AND WASTE DISPOSAL ANNUAL REPORT FOR YEAR 1981 SOLID EFFLUENTS

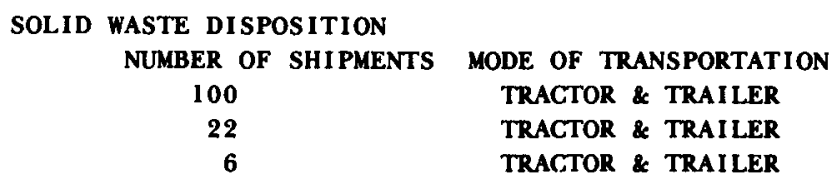

IRRAD I ATED FUEL SHI PMENTS (DI SPOS ITION)

NUMBER OF SHIPMENTS MODE OF TRANSPORTATION

0

ESTIMATE OF MAJOR NUCLIDE COMPOSITION(BY TYPE OF WASTE)

A

ALL OTHERS

CO-60

CS-194

CS-197

OTHER

$\mathrm{ZN}-65$

B

ALL OTHERS

CO-60

CR-51

CS-134

CS-197

OTHER

$\mathrm{ZN}-65$

D

CO-60

CS-137

NB-95

OTHER

ZN-65

ZR-95

TYPE OF WASTE
A. SPENT RESINS, FILTER SLUDGES, EVAPORATOR BOTTOMS, ETC.
B. DRY COMPRESS IBLE WASTE, CONTAMINATED EQU I PMENT, ETC.
C. I RRADIATED COMPONENTS, CONTROL RODS, ETC
D. OTHER

\author{
DESTINATION \\ BARNWELL, S.C. \\ HANFORD, WA \\ RICHLAND, WA
}

DESTINATION

JAN-JUNE JULY-DEC

$\begin{array}{lll}\% & & 2.96 E+01 \\ \% & 2.07 E+00 & 1.89 E+01 \\ \% & 3.53 E+01 & 2.32 E+01 \\ \% & 4.71 E+01 & 2.84 E+01 \\ \% & 7.17 E+00 & \\ \% & 8.36 E+00 & \\ & & \\ \% & & 4.43 E+01 \\ \% & 2.09 E+01 & 1.65 E+01 \\ \% & 2.83 E+00 & \\ \% & 1.88 E+01 & \\ \% & 2.82 E+01 & 1.68 E+01 \\ \% & 1.32 E+01 & \\ \% & 1.62 E+01 & 2.25 E+01\end{array}$

\begin{tabular}{l} 
\% \\
$\% \quad 2.62 \mathrm{E}+01$ \\
$\% \quad 2.45 \mathrm{E}+00$ \\
$\% \quad 6.56 \mathrm{E}+01$ \\
$\%$ \\
$\% \quad 1.75 \mathrm{E}+01$ \\
\hline
\end{tabular}

UNIT YEAR TOTAL

M3 3.04E+02

CI $4.35 \mathrm{E}+03$

M3 6.47E+02

CI $5.88 \mathrm{E}+01$

M3 O.00E+00

CI $0.00 E+00$

M3 3.36E+02

CI $4.63 \mathrm{E}+01$

N/A=NOT APPLICABLE

$N / D=N O T$ DETECTED

N/R=NOT REPORTED 
EFFLUENT AND WASTE DISPOSAL ANNUAL REPORT FOR YEAR 1981 AIRBORNE AND LIQUID EFFLUENTS

UNIT NUMBER : 2

TYPE : $\quad$ BWR

DOCKET NO . : 50-366

COOLING WATER SOURCE : ALTAMAHA RIVER

AIRBORNE EFFLUENTS

NUCLIDES RELEASED

$\mathrm{N}-13$

AR-41

CR-5 1

$\mathrm{MN}-54$

CO-58

FE-59

CO-60

KR-85

KR-85M

KR-87

KR-88

KR-89

SR-89

SR-90

NB-95

ZR-95

SN-113

I-131

XE-131M

I -133

XE-133

XE-133M

CS-134

I-135

XE- 135

$\mathrm{XE}-135 \mathrm{M}$

CS-137

$\mathrm{XE}-137$

$X E-138$

BA-140

LA- 140

CE- 141

CE-144

LIQUID EFFLUENTS

NUCLIDES RELEASED

F-1 8

NA-24

P-32

CR-5 1

MN-54

FE-5 5

MN-56

C0-58
LICENSEE : GEORGIA POWER

LICENSED POWER (MWT) : 2436.00

INITIAL CRITICALITY: 07/04/78

N/A=NOT APPLICABLE

$N / D=N O T$ DETECTED

N/R=NOT REPORTED

\begin{tabular}{|c|}
\hline ACTIVITY (CI) \\
\hline $1.72 E+01$ \\
\hline 5. $96 \mathrm{E}+00$ \\
\hline $2.82 \mathrm{E}-06$ \\
\hline 1. $16 \mathrm{E}-06$ \\
\hline $0.00 E+00$ \\
\hline $0.00 \mathrm{E}+00$ \\
\hline $1.63 \mathrm{E}-05$ \\
\hline $0.00 E+00$ \\
\hline $8.71 E+00$ \\
\hline $5.92 E+00$ \\
\hline $1.19 \mathrm{E}+01$ \\
\hline $0.00 \mathrm{E}+00$ \\
\hline $8.38 \mathrm{E}-05$ \\
\hline $8.66 \mathrm{E}-08$ \\
\hline 1. $55 \mathrm{E}-05$ \\
\hline $2.99 E-07$ \\
\hline $1.65 E-07$ \\
\hline $8.62 \mathrm{E}-03$ \\
\hline $1.25 \mathrm{E}+01$ \\
\hline $6.16 \mathrm{E}-03$ \\
\hline $9.10 E+01$ \\
\hline $1.15 E+00$ \\
\hline $1.21 \mathrm{E}-05$ \\
\hline $2.50 \mathrm{E}-03$ \\
\hline $3.35 E+01$ \\
\hline $2.40 \mathrm{E}+00$ \\
\hline $2.04 \mathrm{E}-05$ \\
\hline $2.72 E+00$ \\
\hline 1. $33 \mathrm{E}+01$ \\
\hline $1.01 \mathrm{E}-04$ \\
\hline 1. $83 \mathrm{E}-04$ \\
\hline $9.69 \mathrm{E}-10$ \\
\hline $1.07 E-07$ \\
\hline ACTIVITY (CI) \\
\hline $1.17 \mathrm{E}-03$ \\
\hline $7.92 \mathrm{E}-03$ \\
\hline $0.00 E+00$ \\
\hline $8.47 E-04$ \\
\hline $6.55 E-04$ \\
\hline $9.09 \mathrm{E}-02$ \\
\hline $0.00 E+00$ \\
\hline $1.18 \mathrm{E}-03$ \\
\hline
\end{tabular}

ACTIVITY (CI)

$5.96 \mathrm{E}+00$

$0.00 \mathrm{E}+0$

1. 63E-0

$0.00 \mathrm{E}+00$

$0.00 \mathrm{E}+00$

$1.25 \mathrm{E}+0$

$6.16 \mathrm{E}-03$

1. 15E+00

$21 \mathrm{E}-05$

. $50 \mathrm{E}-03$

$.04 \mathrm{E}-05$

33E+0

.01E-0

$69 \mathrm{E}-10$ 
EFFLUENT AND WASTE DISPOSAL ANNUAL REPORT FOR YEAR 1981 AIRBORNE AND LIQUID EFFLUENTS

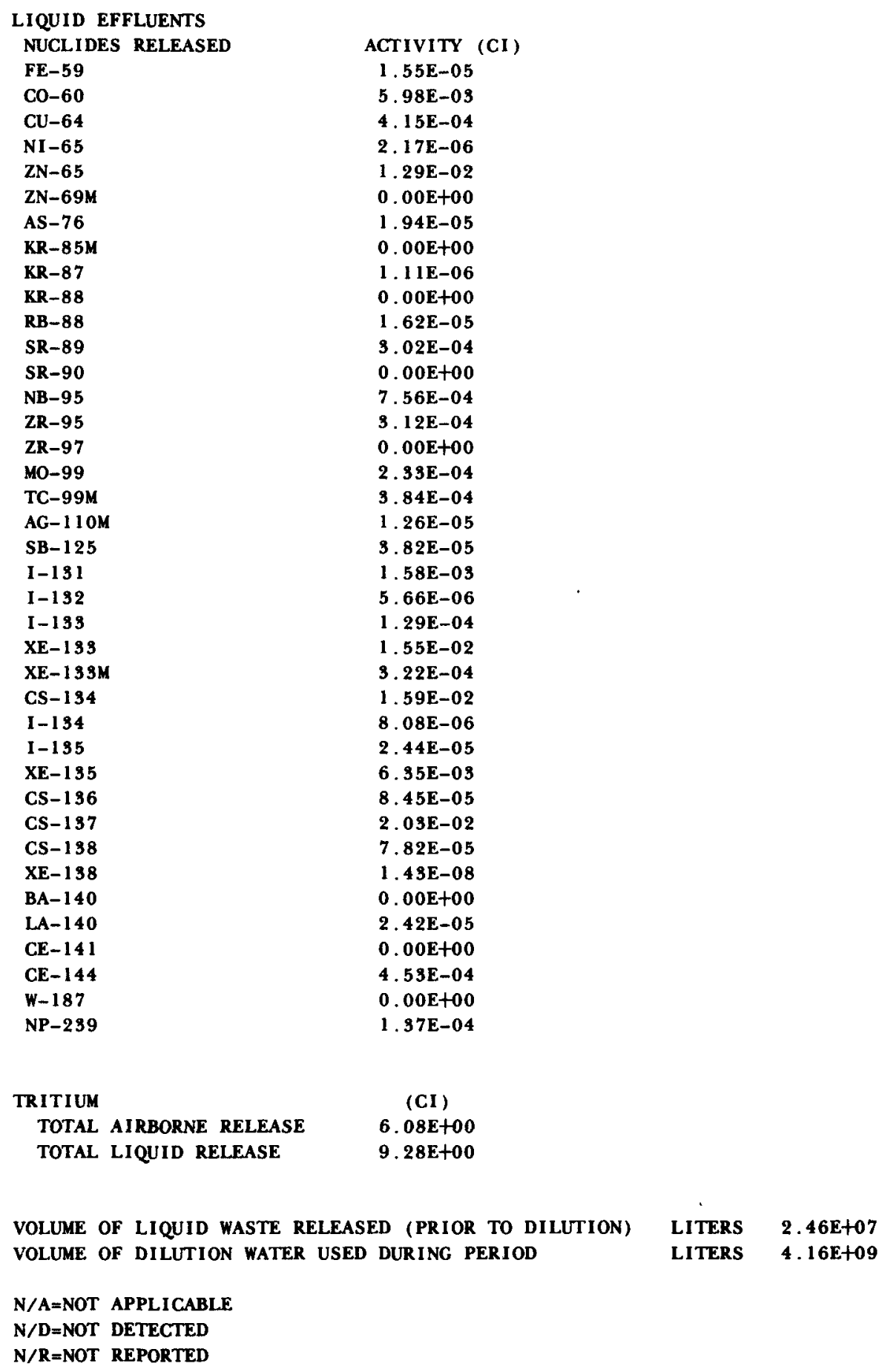


INSTALLATION: EDWIN I. HATCH 2

EFFLUENT AND WASTE DISPOSAL ANNUAL REPORT FOR YEAR 1981 SOLID EFFLUENTS

SOLID WASTE DISPOSITION

NUMBER OF SHIPMENTS MODE OF TRANSPORTATION

$\begin{aligned} 33 & \text { TRACTOR \& TRAILER } \\ 7 & \text { TRACTOR \& TRAILER } \\ 23 & \text { TRUCK OR VAN } \\ 29 & \text { TRUCK OR VAN }\end{aligned}$

DESTINATION

BARNWELL, S.C.

RICHLAND, WA

BARNWELL, S.C.

HANFORD, WA

IRRADIATED FUEL SHI PMENTS (DISPOSITION)

NUMBER OF SHIPMENTS MODE OF TRANSPORTATION

0

DESTINATION

ESTIMATE OF MAJOR NUCLIDE COMPOSITION(BY TYPE OF WASTE)

A

ALL OTHERS

CO-58

$\mathrm{CO}-60$

CR-51

CS-134

CS-137

NB-95

OTHERS

ZN-65

B

ALL OTHERS

CO-60

CR-51

CS-134

CS-137

CS-138

$\mathrm{MN}-54$

OTHERS

SB- 125

$\mathrm{ZN}-65$

TYPE OF WASTE

A. SPENT RESINS, FILTER SLUDGES, EVAPORATOR BOTTOMS, ETC.

B. DRY COMPRESS I BLE WASTE, CONTAMINATED EQUI PMENT, ETC.

C. IRRADIATED COMPONENTS, CONTROL RODS, ETC.

D. OTHER

JAN-JUNE JULY-DEC

*

$\%$

$\%$

$\%$

$\%$

$\%$

$\%$

$\%$

$\%$

8

$\%$

7

$\%$

$\%$

$\%$

$\%$

UNIT YEAR TOTAL

M3 $2,96 \mathrm{E}+02$

CI $2.41 E+02$

M3 $1.11 \mathrm{E}+03$

CI $6.35 \mathrm{E}+01$

MS N/A

CI

M3

CI
$2.24 E+01$

3. $22 \mathrm{E}+01$

8. 12E+00

3. $73 E+01$

4. $43 \mathrm{E}+01$

$1.65 \mathrm{E}+01$

$1.68 \mathrm{E}+01$

1. 65E+00

1. $22 E+00$

.83E+00

$1.61 \mathrm{E}+01$

2. 25E+01

N/A=NOT APPLICABLE

$N / D=N O T$ DETECTED

N/R=NOT REPORTED 
EFFLUENT AND WASTE DISPOSAL ANNUAL REPORT FOR YEAR 1981 AIRBORNE AND LIQUID EFFLUENTS

UNIT NUMBER : 1

TYPE: $\quad$ BWR

DOCKET NO. : 50-139

COOLING WATER SOURCE : HUMBOLDT BAY
LICENSEE : PACIFIC GAS \&ELECTRIC LICENSED POWER (MWT) : 220.00 INITIAL CRITICALITY: $02 / 16 / 63$

\begin{tabular}{|c|c|}
\hline \multicolumn{2}{|l|}{ AI RBORNE EFFLUENTS } \\
\hline MN-54 & N/D \\
\hline $\mathrm{CO}-60$ & $2.96 \mathrm{E}-04$ \\
\hline $\mathbf{K R}-\mathbf{8 3 M}$ & N/D \\
\hline KR-85M & N/D \\
\hline $\mathbf{K R}-87$ & N/D \\
\hline KR-88 & N/D \\
\hline KR-89 & N/D \\
\hline SR-89 & N/D \\
\hline SR-90 & $1.75 E-06$ \\
\hline $\mid-131$ & N/D \\
\hline I-133 & N/D \\
\hline$X E-133$ & $\mathbf{N} / \mathrm{D}$ \\
\hline$X E-133 M$ & N/D \\
\hline $\operatorname{CS}-134$ & N/D \\
\hline $1-135$ & N/D \\
\hline$X E-135$ & N/D \\
\hline XE-1 35M & N/D \\
\hline CS-137 & $1.06 \mathrm{E}-04$ \\
\hline$X E-137$ & N/D \\
\hline$X E-138$ & N/D \\
\hline BA-LA- 140 & N/D \\
\hline CE-144 & N/D \\
\hline \multicolumn{2}{|l|}{ LIQUID EFFLUENTS } \\
\hline NUCLIDES RELEASED & ACTIVITY (CI) \\
\hline CR-51 & N/D \\
\hline MN-54 & $9.68 \mathrm{E}-04$ \\
\hline CO-58 & N/D \\
\hline $\mathrm{CO}-60$ & $5.46 E-02$ \\
\hline $\mathrm{ZN}-65$ & N/D \\
\hline SR-89 & N/D \\
\hline SR-90 & $3.70 E-04$ \\
\hline ZR-NB-95 & N/D \\
\hline MO-99 & N/D \\
\hline TC-99M & N/D \\
\hline$|-13|$ & $\mathrm{N} / \mathrm{D}$ \\
\hline$X E-133$ & $\mathrm{~N} / \mathrm{D}$ \\
\hline CS-134 & $6.25 E-03$ \\
\hline$X E-135$ & N/D \\
\hline CS-137 & $9.17 \mathrm{E}-02$ \\
\hline BA-LA-140 & N/D \\
\hline $\mathrm{CE}-144$ & N/D \\
\hline NP-239 & N/D \\
\hline
\end{tabular}

N/A=NOT APPLICABLE $N / D=$ NOT DETECTED $N / R=$ NOT REPORTED 
EFFLUENT AND WASTE DISPOSAL ANNUAL REPORT FOR YEAR 1981 AIRBORNE AND LIQUID EFFLUENTS

$\begin{array}{lc}\text { TRITIUN } & \text { (CI) } \\ \text { TOTAL AIRBORNE RELEASE } & <4.00 \mathrm{E}-02 \\ \text { TOTAL LIQUID RELEASE } & <1.62 \mathrm{E}-01\end{array}$

VOLUME OF LIQUID WASTE RELEASED (PRIOR TO DILUTION) LITERS 4.49E+05 VOLUME OF DILUTION WATER USED DURING PERIOD

N/A-NOT APPLICABLE N/D=NOT DETECTED N/R-NOT REPORTED 
INSTALLATION : HUMBOLDT BAY

EFFLUENT AND WASTE DISPOSAL ANNUAL REPORT FOR YEAR 1981 SOLID EFFLUENTS

SOLID WASTE DISPOSITION NUMBER OF SHIPMENTS MODE OF TRANSPORTATION

DESTINATION 3

\author{
TRUCK (SOLE USE)
}

IRRADI ATED FUEL SHI PMENTS (DISPOS ITION)

NUMBER OF SHIPMENTS MODE OF TRANSPORTATION 0

ESTIMATE OF MAJOR NUCLIDE COMPOSITION(BY TYPE OF WASTE)

B

CO-60

CS-134

CS-137

MN-54

UNIDENT I FIED
RICHLAND, WA

DESTINATION

JAN-JUNE JULY-DEC

4. 28E+01

2.33E+00

5.12E+01

$9.16 \mathrm{E}-02$

3. $56 \mathrm{E}+00$

UNIT YEAR TOTAL

M3

CI

M3 8.43E+01

CI $5.46 \mathrm{E}-01$

M3

CI

M3

CI 
EFFLUENT AND WASTE DISPOSAL ANNUAL REPORT FOR YEAR 1981 AIRBORNE AND LIQUID EFFLUENTS

UNIT NUMBER: I

TYPE: PWR

DOCKET NO. : 50-3

COOLING WATER SOURCE: HUDSON RIVER

UNIT NUMBER : 2

TYPE: $\quad$ PWR

DOCKET NO. : 50-247

COOLING WATER SOURCE : HUDSON RIVER

AIRBORNE EFFLUENTS

NUCLIDES RELEASED

P-32

CR-5 1

MN-54

FE-55

CO- 57

Co-58

$\mathrm{CO}-60$

$\mathrm{NI}-63$

$\mathrm{ZN}-65$

KR-85

$\mathrm{KR}-85 \mathrm{M}$

KR-87

KR-88

SR-89

SR-90

NB-95

$I-131$

$\mathrm{XE}-191 \mathrm{M}$

I-133

$\mathrm{XE}-133$

XE-133M

CS-134

I -135

XE-1 135

XE-135M

CS-137

XE- 137

XE-1 188

BA-LA-140

LIQUID EFFLUENTS

NUCLIDES RELEASED

P-32

CR-51

MN-54

FE-55

CO-57

CO-58

FE-59

CO-60
LICENSEE : CONSOLIDATED EDISON LICENSED POWER (MWT) : 615.00 INITIAL CRITICALITY:08/02/62

LICENSEE : CONSOLIDATED EDISON LICENSED POWER (MWT) : 2758.00 INITIAL CRITICALITY:05/22/73

N/A=NOT APPLICABLE

N/D $=$ NOT DETECTED

N/R=NOT REPORTED

$$
\begin{aligned}
& \text { ACTIVITY (CI) } \\
& 4.68 E-05 \\
& 7.76 E-05 \\
& 6.14 E-05 \\
& 1.94 E-03 \\
& 1.31 E-05 \\
& 2.67 E-03 \\
& 2.90 E-03 \\
& 2.71 E-04 \\
& 9.82 E-06 \\
& 5.77 E+02 \\
& 5.60 E+01 \\
& 2.49 E+01 \\
& 8.93 E+01 \\
& 5.10 E-05 \\
& 1.45 E-05 \\
& 5.59 E-06 \\
& 3.24 E-02 \\
& 1.33 E+02 \\
& 1.51 E-02 \\
& 7.71 E+03 \\
& 1.05 E+02 \\
& 3.04 E-04 \\
& 4.49 E-02 \\
& 4.57 E+02 \\
& 8.17 E+00 \\
& 2.89 E-03 \\
& 1.07 E+01 \\
& 2.30 E+01 \\
& 5.33 E-04 \\
& \text { ACT1VITY (CI) } \\
& 4.43 E-02 \\
& 2.15 E-01 \\
& 4.98 E-02 \\
& 6.86 E-01 \\
& 6.50 E-03 \\
& 7.86 E-01 \\
& 2.73 E-02 \\
& 1.01 E+00 \\
&
\end{aligned}
$$


EFFLUENT AND WASTE DISPOSAL ANNUAL REPORT FOR YEAR 1981 AIRBORNE AND LIQUID EFFLUENTS

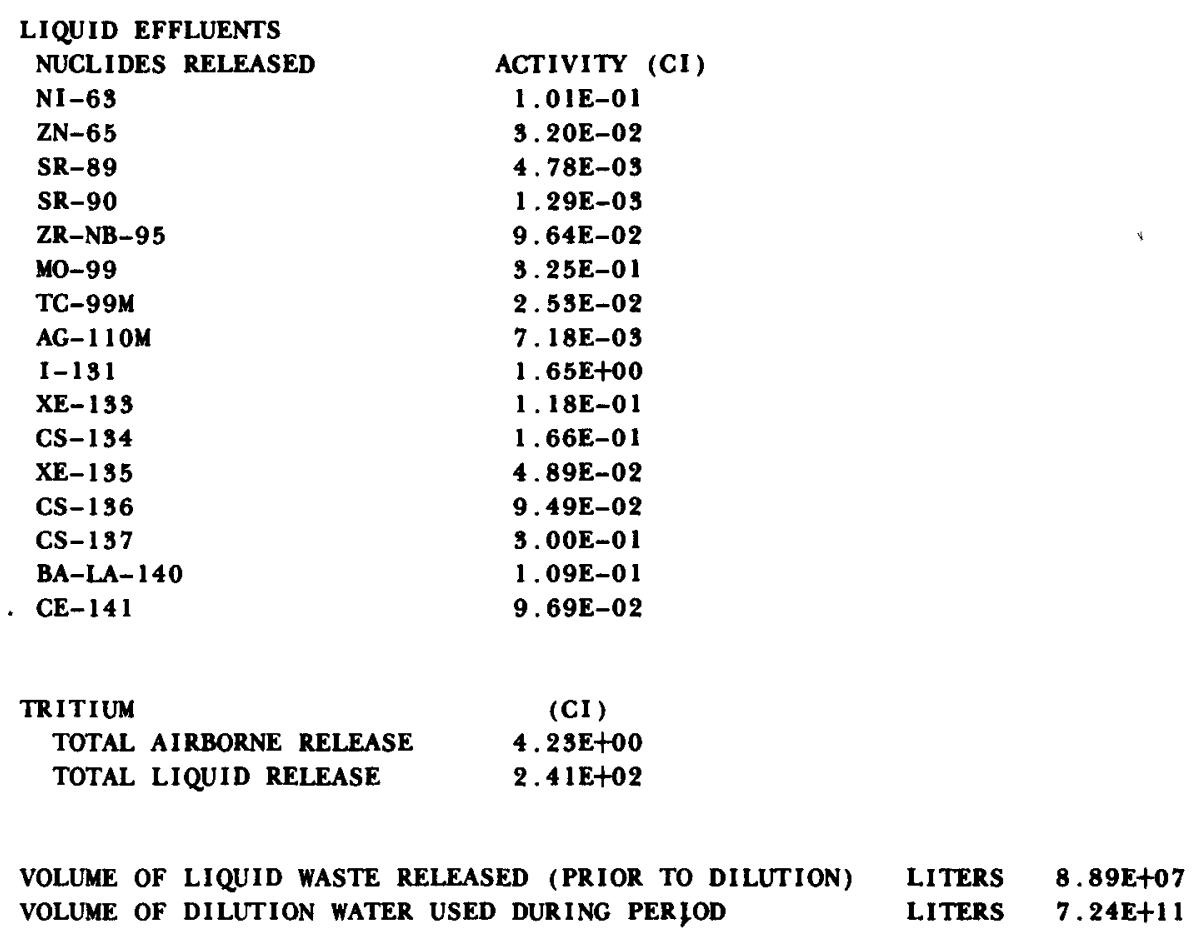

$N / A=N O T$ APPLICABLE

$N / D=N O T$ DETECTED

$N / R=$ NOT REPORTED 
INSTALLATION: INDIAN POINT 1-2

EFFLUENT AND WASTE DISPOSAL ANNUAL REPORT FOR YEAR 1981 SOLID EFFLUENTS

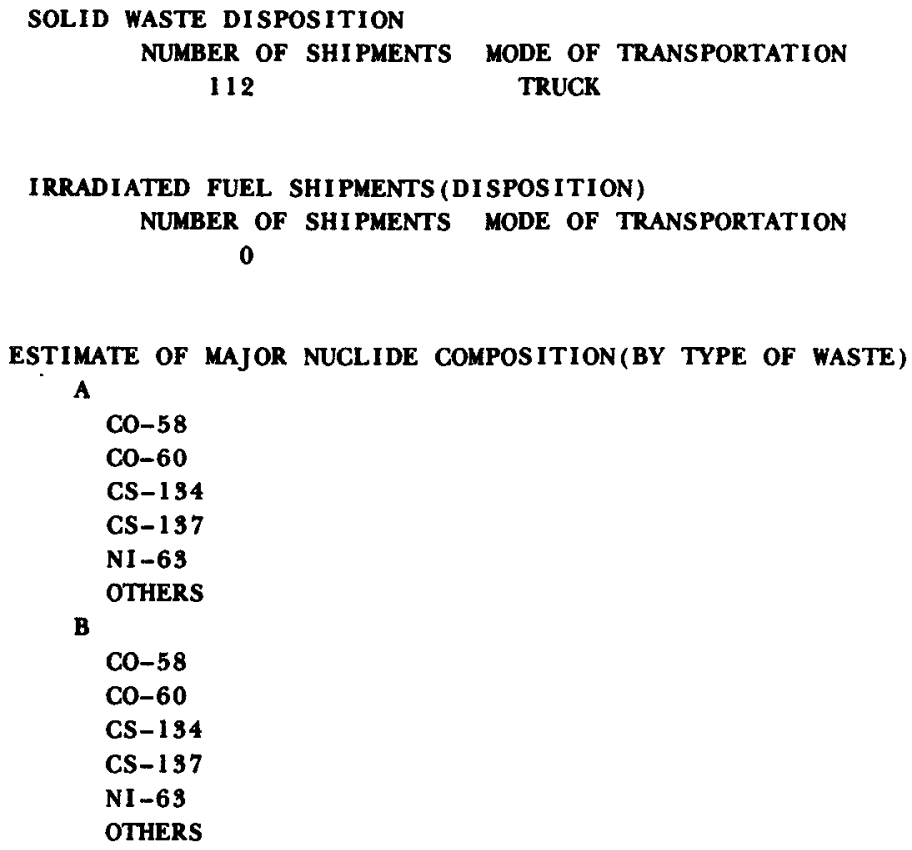

\begin{tabular}{|c|c|c|}
\hline & JAN-JUNE & JULY-DEC \\
\hline$\%$ & $4.60 \mathrm{E}+01$ & $1.90 \mathrm{E}+01$ \\
\hline$\%$ & $1.30 \mathrm{E}+01$ & $3.20 E+01$ \\
\hline$\%$ & $1.20 E+01$ & 1. $.90 E+01$ \\
\hline$\%$ & 1. $70 E+01$ & 3.00E +01 \\
\hline$\%$ & $1.00 E+01$ & \\
\hline$\%$ & $2.00 \mathrm{E}+00$ & \\
\hline$\%$ & $4.60 E+01$ & $2.60 \mathrm{E}+01$ \\
\hline$\%$ & $1.30 \mathrm{E}+01$ & 5.60E+01 \\
\hline * & 1.20E+01 & 5. 70E+00 \\
\hline$\%$ & $1.70 \mathrm{E}+01$ & $1.10 \mathrm{E}+01$ \\
\hline$\%$ & $1.00 E+01$ & \\
\hline$\%$ & $2.00 E+00$ & \\
\hline
\end{tabular}

TYPE OF WASTE
A. SPENT RESINS, FILTER SLUDGES, EVAPORATOR BOTTOMS, ETC.
B. DRY COMPRESSIBLE WASTE, CONTAMINATED EQUI PMENT, ETC.
C. IRRADIATED COMPONENTS, CONTROL RODS, ETC.
D. OTHER

UNIT YEAR TOTAL

$\begin{array}{ll}\text { M3 } & 3.48 E+02 \\ \text { CI } & 1.65 E+03 \\ \text { M3 } & 1.23 E+03 \\ \text { CI } & 5.55 E+01 \\ \text { M3 } & 0.00 E+00 \\ \text { CI } & 0.00 E+00 \\ \text { M3 } & 0.00 E+00 \\ \text { CI } & 0.00 E+00\end{array}$


EFFLUENT AND WASTE DISPOSAL ANNUAL REPORT FOR YEAR 1981 AIRBORNE AND LIQUID EFFLUENTS

UNIT NUMBER: 3

TYPE : PWR

DOCKET NO . : 50-286

COOLING WATER SOURCE:

AIRBORNE EFFLUENTS

NUCLIDES RELEASED

P-32

CR-5 1

MN-54

FE-55

CO-57

CO-58

CO-60

NI -63

ZN-65

KR-85

KR-85M

SR-85

KR-87

KR-88

SR-89

SR-90

NB-95

CD-109

SN-113

I -131

XE- $131 \mathrm{M}$

BA-1 33

I -133

XE-193

$\mathrm{XE}-133 \mathrm{M}$

CS-134

I -195

XE-135

$\mathrm{XE}-195 \mathrm{M}$

CS-1 137

XE-1 138

CE-1 39

BA-L.A- 140

CE-141

CE-144

HG-203

LIQUID EFFLUENTS

NUCLIDES RELEASED

NA-24

P-32

CR-51

MN-54

FE-55

N/A=NOT APPLICABLE

$N / D=N O T$ DETECTED

$N / R=N O T$ REPORTED
LICENSEE : POWER AUTHORITY OF THE STATE OF NEW YORK

LICENSED POWER (MWT) $: 2760.00$

INITIAL CRITICALITY : 04/06/76

$$
\begin{aligned}
& \text { ACTIVITY (CI) } \\
& \text { 3.26E-07 } \\
& 7.38 E-06 \\
& 1.31 E-06 \\
& 8.09 E-05 \\
& 1.07 E-06 \\
& 7.98 E-05 \\
& 3.65 E-05 \\
& 2.29 E-05 \\
& 1.91 E-06 \\
& 2.86 E+02 \\
& 8.20 E+01 \\
& 2.37 E-07 \\
& 2.96 E+00 \\
& 6.22 E+00 \\
& 4.13 E-07 \\
& 5.60 E-08 \\
& 6.52 E-07 \\
& 1.76 E-05 \\
& 2.57 E-07 \\
& 1.66 E-03 \\
& 8.26 E+01 \\
& 1.64 E-06 \\
& 3.75 E-05 \\
& 5.43 E+03 \\
& 5.24 E+01 \\
& 1.79 E-05 \\
& 0.00 E+00 \\
& 6.16 E+02 \\
& 1.31 E+01 \\
& 3.13 E-05 \\
& 2.11 E-02 \\
& 1.22 E-06 \\
& 1.90 E-06 \\
& 4.63 E-07 \\
& 4.08 E-06 \\
& 5.34 E-07 \\
& \text { ACTIVITY (CI) } \\
& 9.94 E-04 \\
& 1.33 E-02 \\
& 5.61 E-02 \\
& 7.43 E-02 \\
& 6.89 E-01 \\
&
\end{aligned}
$$


EFFLUENT AND WASTE DISPOSAL ANNUAL REPORT FOR YEAR 1981 AIRBORNE AND LIQUID EFFLUENTS

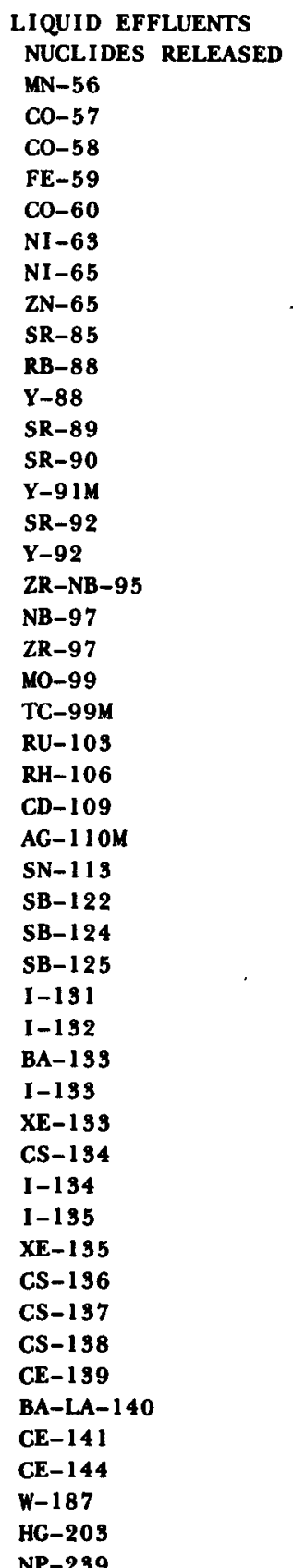

NP-239

N/A =NOT APPLICABLE N/D=NOT DETECTED N/R=NOT REPORTED

ACTIVITY (CI)
$2.67 E-03$
$2.46 E-03$
$8.90 E-01$
$1.26 E-02$
$3.15 E-01$
$1.71 E-01$
$1.93 E-05$
$2.22 E-03$
$1.03 E-04$
$5.52 E-04$
$3.07 E-05$
$4.58 E-04$
$7.40 E-04$
$1.55 E-03$
$1.81 E-03$
$7.31 E-04$
$8.07 E-03$
$3.61 E-02$
$6.71 E-05$
$8.68 E-03$
$1.34 E-03$
$2.52 E-04$
$2.61 E-03$
$2.25 E-03$
$1.60 E-02$
$1.71 E-04$
$5.58 E-03$
$2.45 E-02$
$7.20 E-02$
$1.98 E-02$
$4.27 E-03$
$1.26 E-03$
$1.29 E-02$
$2.84 E+00$
$5.72 E-02$
$2.55 E-03$
$6.45 E-03$
$1.27 E-01$
$1.45 E-04$
$9.19 E-02$
$1.31 E-03$
$3.51 E-04$
$7.19 E-03$
$1.64 E-03$
$1.59 E-04$
$8.43 E-05$
$2.16 E-04$
$3.09 E-04$


EFFLUENT AND WASTE DISPOSAL ANNUAL REPORT FOR YEAR 1981 AIRBORNE AND LIQUID EFFLUENTS

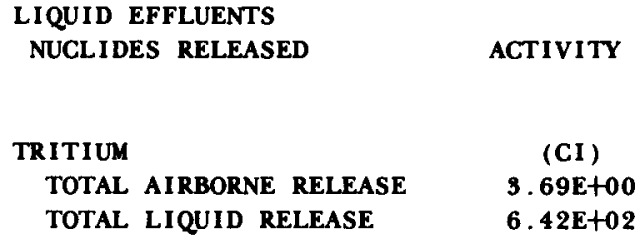

VOLUME OF LIQUID WASTE RELEASED (PRIOR TO DILUTION)

LITERS $3.71 E+07$

VOLUME OF DILUTION WATER USED DURING PERIOD

LITERS 7.25E+11

N/A=NOT APPLICABLE

$N / D=N O T$ DETECTED

N/R=NOT REPORTED 
INSTALLATION: INDIAN POINT 3

EFFLUENT AND WASTE DISPOSAL ANNUAL REPORT FOR YEAR 1981 SOLID EFFLUENTS

SOLID WASTE DISPOSITION NUMBER OF SHI PMENTS 20
MODE OF TRANSPORTATION TRUCK

TRUCK
DESTINATION

BARNWELL, S.C.

RICHLAND, WA

DESTINATION

IRRADIATED FUEL SHI PMENTS (DISPOS ITION)

NUMBER OF SHIPMENTS MODE OF TRANSPORTATION

ESTIMATE OF MAJOR NUCLIDE COMPOSITION(BY TYPE OF WASTE)

$\mathbf{A}$

AG-110M
CO-58
CO-60
CS-134
CS-197
$M N-54$
CO-58
CO-60
$C R-51$
$C S-134$
$C S-197$
$M N-54$

TYPE OF WASTE

A. SPENT RESINS, FILTER SLUDGES, EVAPORATOR BOTTOMS, ETC

B. DRY COMPRESSIBLE WASTE, CONTAMINATED EQU I PMENT, ETC.

C. IRRADIATED COMPONENTS, CONTROL RODS, ETC.

D. OTHER
JAN-JUNE JULY-DEC
4.00E+00
1. 70E+01
4. $21 \mathrm{E}+01$
$6.50 \mathrm{E}+01$
$2.44 \mathrm{E}+01$
$1.17 \mathrm{E}+01$
$7.00 \mathrm{E}+00 \quad 2.05 \mathrm{E}+01$
$7.00 \mathrm{E}+00 \quad 1.30 \mathrm{E}+00$
3. 50E+01 3.21E+01
3. $60 \mathrm{E}+01$ 5. 10E+01
3. $00 \mathrm{E}+00$
8.00E+00 1.90E+00
$1.60 \mathrm{E}+01 \quad 7.70 \mathrm{E}+00$
5. $00 E+00 \quad 4.30 E+00$

UNIT YEAR TOTAL

M3 4.93E+01

CI 5.75E+01

M3 2.68E+02

CI $6.50 E+00$

M3

CI

MS

CI 
EFFLUENT AND WASTE DISPOSAL ANNUAL REPORT FOR YEAR 1981 AIRBORNE AND LIQUID EFFLUENTS

UNIT NUMBER: 1

TYPE : PWR DOCKET NO, : 50-305 COOLING WATER SOURCE : LAKE MICHIGAN

\section{AIRBORNE EFFLUENTS}

NUCLIDES RELEASED

NA-24

AR-41

C0-60

KR-85M

SR-89

SR-90

I-131

I-132

I-133

XE-133

XE-139M

CS-134

I-134

I-135

XE- 135

CS- 137

RN-222

UN I DENT I F IED

\section{LIQUID EFFLUENTS}

NUCLIDES RELEASED

NA-24

CR-51

MN-54

CO-57

CO-58

FE-59

CO-60

KR-85M

SR-89

SR-90

NB-95

ZR-95

AG-110M

SN-113

SN-117M

SB-1 24

SB-1 25

I-131

XE-133

CS-134

CS-137

N/A=NOT APPLICABLE

N/D=NOT DETECTED

N/R=NOT REPORTED
LICENSEE:WISCONSIN PUBLIC SERVICE LICENSED POWER (MWT) : 1650.00 INITIAL CRITICALITY: 03/07/74
ACTIVITY (CI)

$1.07 \mathrm{E}-06$

$1.63 \mathrm{E}+00$

1. 26E-05

2.38E-02

$<5.98 E-06$

$<5.98 E-06$

8. 63E-05

1. 15E-05

6.96E-05

3. 32E+00

1. $78 \mathrm{E}+00$

2.71E-07

$6.25 \mathrm{E}-07$

2.52E-05

8.34E-01

1. $00 \mathrm{E}-05$

6.32E-04

$<1.11 E+02$

ACTIVITY (CI)

3. 11E-03

1. 43E-02

9. 22E-03

2.98E-04

4. 37E-01

4.53E-04

2. $11 \mathrm{E}-01$

2. 40E-05

1. 04E-04

<4. 45E-05

7. 44E-04

3. 84E-04

2.39E-02

8. 80E-05

7. 65E-05

2. 23E-02

1. $72 \mathrm{E}-02$

1. $02 \mathrm{E}-06$

5. 25E-05

2. 09E-02

4. $96 \mathrm{E}-02$ 
EFFLUENT AND WASTE DISPOSAL ANNUAL REPORT FOR YEAR 1981 AIRBORNE AND LIQUID EFFLUENTS

$\begin{array}{lc}\text { TRITIUM } & \text { (CI) } \\ \text { TOTAL AIRBORNE RELEASE } & \mathbf{3 . 8 2 E + 0 0} \\ \text { TOTAL LIQUID RELEASE } & 2.51 \mathrm{E}+02\end{array}$

VOLUME OF LIQUID WASTE RELEASED (PRIOR TO DILUTION) LITERS 6.24E+06 VOLUME OF DILUTION WATER USED DURING PERIOD 
INSTALLATION : KEWAUNEE

EFFLUENT AND WASTE DISPOSAL ANNUAL REPORT FOR YEAR 1981 SOLID EFFLUENTS

SOLID WASTE DISPOSITION

NUMBER OF SHIPMENTS MODE OF TRANSPORTATION

$$
14
$$

SHIELDED VAN

IRRADIATED FUEL SHI PMENTS (DISPOSITION)

NUMBER OF SHIPMENTS MODE OF TRANSPORTATION

$\mathbf{0}$

ESTIMATE OF MAJOR NUCLIDE COMPOSITION(BY TYPE OF WASTE)

A

$\mathrm{CO}-57$

CO-58

C0-60

CS- 134

CS-137

$M N-54$

SB-125

B

CO-58

CO-60

CS-134

CS-137

MN-54

CO-57

CO-58

C0-60

CS-134

CS-137

MN-54

SB-125

D

C0-57

Co-58

C0-60

CS-134

CS-137

MN-54

SB-125
DESTINATION

BARNWELL, S.C.

DESTINATION

JAN-JUNE JULY-DEC
3. 14E+01
5. $16 \mathrm{E}+01$
3. 27E+01
$3.40 E+00$
$4.84 \mathrm{E}+01$
1. $15 \mathrm{E}+01$
$3.81 \mathrm{E}+00$
1. $29 \mathrm{E}+01$
2. 10E+00
$1.68 \mathrm{E}+00$
4. 90E-01

5. 00E-02

1. 08E+01

3. $58 \mathrm{E}+01$

$6.86 \mathrm{E}+01$

4. $78 \mathrm{E}+01$

3. 80E+00

$3.40 E+00$

1. 29E+0I

$1.12 \mathrm{E}+01$

3. 90E +00

1. 80E+00

$1.12 \mathrm{E}+01$

2.00E-01

6. 84E+01

$1.99 \mathrm{E}+01$

3. $70 E+00$

5. $11 \mathrm{E}+01$

5. 70E+00

1. 29E+01

1. $99 \mathrm{E}+01$

3. $80 \mathrm{E}+00$

1. 20E+00

2. $20 \mathrm{E}+00$

$1.56 \mathrm{E}+01$

2.00E-02

$3.42 \mathrm{E}+01$

$6.48 E+01 \quad 4.82 E+01$

3.70E+00 3.63E+00

1. 25E+01 $1.21 \mathrm{E}+01$

$3.40 E+00 \quad 1.77 E+00$

2. 20E-01

N/A $=$ NOT APPLICABLE

$N / D=$ NOT DETECTED

$N / R=$ NOT REPORTED 
INSTALLATION : KEWAUNEE

EFFLUENT AND WASTE DISPOSAL ANNUAL REPORT FOR YEAR 1981 SOLID EFFLUENTS

TYPE OF WASTE

A. SPENT RESINS, FILTER SLUDGES, EVAPORATOR

UNIT YEAR TOTAL

. SPENT RESINS

1.02E+O

B. DRY COMPRESS IBLE WASTE, CONTAMINATED

CI 1.43E+02

M3 2.04E+01

EQUI PMENT, ETC.

CI 4.14E+00

C. IRRADIATED COMPONENTS, CONTROL RODS, ETC.

D. OTHER

CONTAMINATED FILTER

M3 2.99E+0

CI $1.69 \mathrm{E}+00$

M3 1.34E+O 1

CI $4.97 \mathrm{E}+01$

N/A=NOT APPLICABLE

$N / D=N O T$ DETECTED

$N / R=N O T$ REPORTED 
EFFLUENT AND WASTE DISPOSAL ANNUAL REPORT FOR YEAR 1981 AIRBORNE AND LIQUID EFFLUENTS

\begin{tabular}{lc} 
LIQUID EFFLUENTS & \\
NUCLIDES RELEASED & ACTIVITY (CI) \\
CO-57 & $2.36 E-03$ \\
CO-58 & $1.89 E-03$ \\
FE-59 & $2.42 E-02$ \\
CO-60 & $8.33 E-01$ \\
ZN-65 & $2.86 E-02$ \\
KR-85M & $6.95 E-05$ \\
KR-87 & $3.64 E-05$ \\
KR-88 & $4.41 E-05$ \\
RB-88 & $2.53 E-05$ \\
SR-89 & $8.54 E-03$ \\
SR-90 & $4.59 E-03$ \\
SR-91 & $7.54 E-04$ \\
SR-92 & $4.82 E-04$ \\
NB-95 & $1.52 E-02$ \\
ZR-95 & $7.41 E-03$ \\
MO-99 & $2.01 E-03$ \\
TC-99M & $7.89 E-03$ \\
RU-103 & $7.20 E-03$ \\
RU+RH-105 & $9.21 E-04$ \\
RU+RH-106 & $1.69 E-02$ \\
I-131 & $1.83 E-03$ \\
XE-131M & $4.67 E-03$ \\
I-132 & $7.79 E-05$ \\
I-133 & $4.12 E-03$ \\
XE-133 & $1.75 E-03$ \\
XE-133M & $2.86 E-04$ \\
CS-134 & $8.24 E-02$ \\
I-135 & $1.63 E-03$ \\
XE-135 & $1.59 E-03$ \\
XE-135M & $8.86 E-04$ \\
CS-137 & $6.97 E-01$ \\
BA-139 & $9.79 E-05$ \\
BA+LA-140 & $1.44 E-03$ \\
CE-141 & $3.20 E-03$ \\
CE-144 & $3.67 E-02$ \\
NP-239 & $4.68 E-03$ \\
TRITIUM & \\
TOTAL AIRBORNE RELEASE & (CI) \\
TOTAL LIQUID RELEASE & $7.74 E+01$ \\
& \\
\hline & \\
\hline
\end{tabular}

VOLUME OF LIQUID WASTE RELEASED (PRIOR TO DILUTION) LITERS 1.43E+05 VOLUME OF DILUTION WATER USED DURING PERIOD 
INSTALLATION : LACROSSE

EFFLUENT AND WASTE DISPOSAL ANNUAL REPORT FOR YEAR 1981 SOLID EFFLUENTS

SOLID WASTE DISPOSITION NUMBER OF SHIPMENTS MODE OF TRANSPORTATION 2 TRUCK

\section{DESTINATION}

BARNWELL, S.C.

ESTIMATE OF MAJOR NUCLIDE COMPOS ITION(BY TYPE OF WASTE)

A

CE-144

C0-57

C0-58

C0-60

CS-134

CS- 137

MN-54

NB-95

NB-97

ZN-65

JAN-JUNE JULY-DEC

9. 12E-01

2. 12E-01

4. $65 \mathrm{E}+00$

6. $75 E+01$

2.18E+00

1. 27E+01

6. $12 \mathrm{E}+00$

1. 20E-02

1. $33 \mathrm{E}+00$

$4.42 E+00$

TYPE OF WASTE

A. SPENT RESINS, FILTER SLUDGES, EVAPORATOR BOTTOMS, ETC.

B. DRY COMPRESS IBLE WASTE, CONTAMINATED EQU I PMENT, ETC.

C. IRRADIATED COMPONENTS, CONTROL RODS, ETC.

D. OTHER

UNIT YEAR TOTAL

M3 $\quad 4.82 E+00$

CI 6.11E+01

M3

CI

M3

CI

M3

CI

N/A $=$ NOT APPLICABLE

$N / D=N O T$ DETECTED

N/R=NOT REPORTED 
EFFLUENT AND WASTE DISPOSAL ANNUAL REPORT FOR YEAR 1981 AIRBORNE AND LIQUID EFFLUENTS

UNIT NUMBER: 1

TYPE: PWR

DOCKET NO . : 50-309

COOLING WATER SOURCE: BACK RIVER
LICENSEE : MAINE YANKEE ATOMIC POWER

LICENSED POWER (MWT) : 2630.00

INITIAL CRITICALITY : 10/23/72

\begin{tabular}{|c|c|}
\hline \multicolumn{2}{|l|}{ AIRBORNE EFFLUENTS } \\
\hline NUCLIDES RELEASED & ACTIVITY (CI) \\
\hline AR-41 & N/D \\
\hline CO-58 & $3.92 \mathrm{E}-05$ \\
\hline $\mathrm{CO}-60$ & $8.98 E-05$ \\
\hline $\mathbf{K R}-85$ & 1. $17 \mathrm{E}+02$ \\
\hline KR-85M & N/D \\
\hline KR-87 & N/D \\
\hline KR-88 & N/D \\
\hline SR-89 & $1.36 E-06$ \\
\hline SR-90 & $5.83 \mathrm{E}-08$ \\
\hline I-131 & $4.20 E-04$ \\
\hline $\mathrm{XE}-131 \mathrm{M}$ & 3. $80 E+00$ \\
\hline I-132 & N/D \\
\hline $1-133$ & 1. $18 \mathrm{E}-03$ \\
\hline$X E-133$ & 1.99E+02 \\
\hline$X E-133 M$ & $1.53 \mathrm{E}-01$ \\
\hline CS-194 & 5. $19 \mathrm{E}-06$ \\
\hline$I-134$ & N/D \\
\hline$I-135$ & N/D \\
\hline $\mathrm{XE}-135$ & 8. $16 E+00$ \\
\hline $\mathrm{XE}-135 \mathrm{M}$ & N/D \\
\hline CS-137 & 3. $17 \mathrm{E}-05$ \\
\hline$X E-138$ & $\mathrm{~N} / \mathrm{D}$ \\
\hline$B A-140$ & N/D \\
\hline UNIDENT I F IED & 2. $11 \mathrm{E}-04$ \\
\hline \multicolumn{2}{|l|}{ LIQUID EFFLUENTS } \\
\hline NUCLIDES RELEASED & ACTIVITY (CI) \\
\hline$F-18$ & $5.81 \mathrm{E}-03$ \\
\hline NA-24 & $1.62 \mathrm{E}-03$ \\
\hline CR-51 & N/D \\
\hline$M N-54$ & $8.63 E-03$ \\
\hline CO-57 & 1. $16 \mathrm{E}-04$ \\
\hline $\mathrm{CO}-58$ & $9.54 \mathrm{E}-02$ \\
\hline FE-59 & $\mathrm{N} / \mathrm{D}$ \\
\hline $\mathrm{CO}-60$ & $3.65 E-02$ \\
\hline $\mathrm{ZN}-65$ & N/D \\
\hline SE-75 & $1.52 \mathrm{E}-01$ \\
\hline SR-89 & $1.96 \mathrm{E}-04$ \\
\hline SR-90 & $3.92 E-05$ \\
\hline ZR-95 & N/D \\
\hline MO-99 & $4.43 E-05$ \\
\hline TC-99M & N/D \\
\hline$A G-110 M$ & $8.08 E-04$ \\
\hline$S B-124$ & 5.96E-04 \\
\hline \multicolumn{2}{|l|}{ N/A $=$ NOT APPLICABLE } \\
\hline \multicolumn{2}{|l|}{ N/D=NOT DETECTED } \\
\hline$N / R=N O T$ REPORTED & \\
\hline
\end{tabular}


EFFLUENT AND WASTE DISPOSAL ANNUAL REPORT FOR YEAR 1981 AIRBORNE AND LIQUID EFFLUENTS

$\begin{array}{lc}\text { LIQUID EFFLUENTS } & \\ \text { NUCLIDES RELEASED } & \text { ACTIVITY (GI) } \\ \text { SB-125 } & 3.39 E-04 \\ \text { I-131 } & 1.76 E-02 \\ \text { I-133 } & 1.43 E-03 \\ \text { XE-133 } & 5.26 E-03 \\ \text { CS-134 } & 6.22 E-03 \\ \text { XE-135 } & 1.78 E-03 \\ \text { CS-137 } & 5.07 E-02 \\ \text { BA-140 } & \text { N/D } \\ \text { CE-141 } & 1.56 E-05 \\ \text { CE-143 } & 6.64 E-05 \\ \text { HG-203 } & 4.97 E-02 \\ \text { UNIDENTIFIED } & 5.70 E-03 \\ & \\ \text { TRITIUM } & (\mathrm{CI}) \\ \text { TOTAL AIRBORNE RELEASE } & 4.48 E+00 \\ \text { TOTAL LIQUID RELEASE } & 2.16 E+02\end{array}$

VOLUME OF LIQUID WASTE RELEASED (PRIOR TO DILUTION)
VOLUME OF DILUTION WATER USED DURING PERIOD 
EFFLUENT AND WASTE DISPOSAL ANNUAL REPORT FOR YEAR 1981 SOLID EFFLUENTS

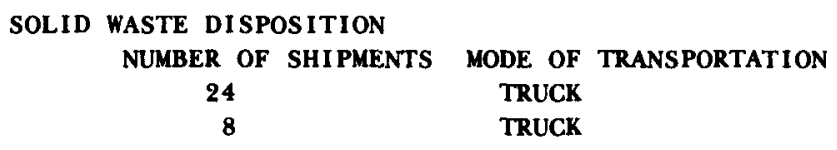

I RRADI ATED FUEL. SHI PMENTS (DI SPOS ITION)

NUMBER OF SHIPMENTS MODE OF TRANSPORTATION 0

ESTIMATE OF MAJOR NUCLIDE COMPOS ITION(BY TYPE OF WASTE)

A

CO-58

$\mathrm{CO}-60$

CS-137

B

CO-58

$\mathrm{CO}-60$

CS-1 197
DESTINATION

BARNWELL, S.C.

RICHLAND, WA

DESTINATION

JAN-JUNE JULY-DEC

$\begin{array}{lll}* & 2.50 E+01 & 2.50 E+01 \\ * & 2.50 E+01 & 2.50 E+01 \\ \text { \% } & 5.00 E+01 & 5.00 E+01 \\ & & \\ \text { \% } & 2.50 E+01 & 2.50 E+01 \\ \text { \% } & 2.50 E+01 & 2.50 E+01 \\ \text { \% } & 5.00 E+01 & 5.00 E+01\end{array}$

TYPE OF WASTE

A. SPENT RESINS, FILTER SLUDGES, EVAPORATOR BOTTOMS, ETC.

B. DRY COMPRESSIBLE WASTE, CONTAMINATED EQUI PMENT, ETC.

C. IRRADIATED COMPONENTS, CONTROL RODS, ETC.

D. OTHER

UNIT YEAR TOTAL.

M3 $1.49 E+02$

CI $\quad 1.65 \mathrm{E}+03$

M3 $\quad 2.65 \mathrm{E}+02$

CI $1.53 E+01$

M3

CI

M3

CI 
EFFLUENT AND WASTE DISPOSAL ANNUAL REPORT FOR YEAR 1981 AIRBORNE AND LIQUID EFFLUENTS

UNIT NUMBER : 1

TYPE : PWR

DOCKET NO. : 50-369

COOLING WATER SOURCE:LAKE NORMAN

\section{AIRBORNE EFFLUENTS}

NUCLIDES RELEASED

AR-41

KR-85

KR-85M

SR-90

$\mathrm{XE}-133$

$\mathrm{XE}-133 \mathrm{M}$

$\mathrm{XE}-135$

\section{LIQUID EFFLUENTS}

NUCL I DES RELEASED

$\mathrm{NA}-24$

AR-4 1

CR-51

MN-54

$\mathrm{CO}-57$

CO-58

FE-59

$\mathrm{CO}-60$

SR-89

SR-92

NB-95

ZR-95

MO-99

TC-99M

AG-110M

SB- 122

SB- 124

I-131

I -133

XE-1 33

$X E-135$

CE-144

W-187

TRITIUM

TOTAL AIRBORNE RELEASE

TOTAL LIQUID RELEASE

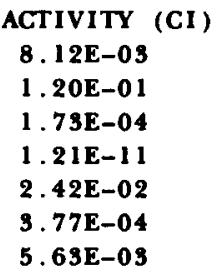

2. 42E-02

3. $77 \mathrm{E}-04$

5.63E-03

LICENSEE : DUKE POWER CO

LICENSED POWER (MWT): 3411.00

INITIAL CRITICALITY:08/08/81

$$
\begin{aligned}
& \text { ACTIVITY (CI) } \\
& \text { 8.83E-03 } \\
& 8.99 E-04 \\
& 4.86 E-02 \\
& 1.24 E-02 \\
& 7.14 E-06 \\
& 2.35 E-01 \\
& 2.17 E-03 \\
& 3.51 E-02 \\
& 9.52 E-04 \\
& 8.00 E-04 \\
& 6.80 E-04 \\
& 5.67 E-04 \\
& 3.13 E-04 \\
& 1.66 E-03 \\
& 1.32 E-03 \\
& 2.87 E-04 \\
& 3.09 E-03 \\
& 8.89 E-04 \\
& 1.26 E-03 \\
& 2.07 E-04 \\
& 2.69 E-04 \\
& \text { 3.42E-03 } \\
& \text { 3.58E-03 }
\end{aligned}
$$

VOLUME OF LIQUID WASTE RELEASED (PRIOR TO DILUTION)

N/A=NOT APPLICABLE

N/D $=$ NOT DETECTED

N/R=NOT REPORTED 
INSTALLATION : MCGUIRE

EFFLUENT AND WASTE DISPOSAL ANNUAL REPORT FOR YEAR 1981 SOLID EFFLUENTS

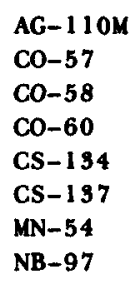

A. SPENT RESINS, FILTER SLUDGES, EVAPORATOR BOTTOMS, ETC.

B. DRY COMPRESS IBLE WASTE, CONTAMINATED EQUI PMENT, ETC.

C. IRRADIATED COMPONENTS, CONTROL RODS, ETC.

D. OTHER

DESTINATION BARNWELL SC

JAN-JUNE JULY-DEC

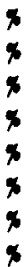

5. 10E-01

1. $74 \mathrm{E}+0 \mathrm{I}$

1. $80 \mathrm{E}+00$

3. 58E+01

1.97E+01

2.37E+01

1. 20E-01

6. 20E-01
UNIT YEAR TOTAL

M3

CI

M3 1.98E+01

CI $1.31 \mathrm{E}-01$

M3

CI

M3

CI 
EFFLUENT AND WASTE DISPOSAL ANNUAL REPORT FOR YEAR 1981 AIRBORNE AND LIQUID EFFLUENTS

UNIT NUMBER: 1
TYPE: $\quad$ BWR

DOCKET NO . :50-245

COOLING WATER SOURCE : LONG ISLAND SOUND

\section{AIRBORNE EFFLUENTS}

NUCLID́ES RELEASED

CR-51

MN -54

C0-58

FE-59

$\mathrm{CO}-60$

KR-83M

KR-85

KR-85M

KR-87

KR-88

KR-89

SR-89

SR-90

I-131

XE-131M

I-133

$\mathrm{XE}-133$

XE-139M

CS- 134

I-135

$X E-135$

XE-135M

CS- 137

XE- 137

XE- 138

BA-LA-140

CE-141

\section{LIQUID EFFLUENTS}

NUCLIDES RELEASED

NA-24

CR-5 1

MN-54

CO-58

CO-60

SR-89

SR-90

MO-99

TC-99M

SB-124

I -131

I-133

$\mathrm{XE}-133$

CS-134
LICENSEE : NORTHEAST NUCLEAR ENERGY

LICENSED POWER (MWT):2011.00

INITIAL CRITICALITY : $10 / 26 / 70$

N/A $=$ NOT APPLICABLE

N/D $=$ NOT DETECTED

$N / R=N O T$ REPORTED

$$
\begin{aligned}
& \text { ACTIVITY (CI) } \\
& 6.39 E-03 \\
& 7.76 E-04 \\
& 1.79 E-04 \\
& 8.02 E-06 \\
& 5.18 E-03 \\
& 4.62 E+02 \\
& 5.94 E-03 \\
& 6.68 E+02 \\
& 2.51 E+03 \\
& 2.44 E+03 \\
& 1.12 E+00 \\
& 6.24 E-03 \\
& 7.17 E-05 \\
& <7.52 E-02 \\
& 2.44 E-01 \\
& 3.88 E-01 \\
& 2.70 E+02 \\
& 1.21 E+01 \\
& 2.90 E-05 \\
& 5.86 E-01 \\
& 2.09 E+03 \\
& 1.10 E+03 \\
& 8.29 E-04 \\
& 7.95 E+00 \\
& 4.76 E+03 \\
& <4.88 E-02 \\
& 1.08 E-04 \\
&
\end{aligned}
$$

ACTIVITY (CI)

$$
\begin{array}{r}
1.14 \mathrm{E}-04 \\
4.10 \mathrm{E}-04 \\
2.77 \mathrm{E}-02 \\
6.08 \mathrm{E}-04 \\
2.00 \mathrm{E}-01 \\
<2.26 \mathrm{E}-03 \\
4.75 \mathrm{E}-04 \\
5.62 \mathrm{E}-05 \\
8.00 \mathrm{E}-05 \\
1.96 \mathrm{E}-02 \\
<6.83 \mathrm{E}-04 \\
9.34 \mathrm{E}-06 \\
8.42 \mathrm{E}-03 \\
2.18 \mathrm{E}-02
\end{array}
$$


EFFLUENT AND WASTE DISPOSAL ANNUAL REPORT FOR YEAR 1981 AIRBORNE AND LIQUID EFFLUENTS

$\begin{array}{lc}\text { LIQUID EFFLUENTS } & \\ \text { NUCLIDES RELEASED } & \text { ACTIVITY (CI) } \\ \text { XE-135 } & <3.16 E-02 \\ \text { CS-137 } & 1.21 E-01 \\ \text { BA-LA-140 } & <8.92 E-04 \\ \text { CE-141 } & 7.57 E-05 \\ \text { CE-144 } & 9.92 E-04 \\ & \\ \text { TRITIUM } & (\mathrm{CI}) \\ \text { TOTAL AIRBORNE RELEASE } & 9.47 E+01 \\ \text { TOTAL LIQUID RELEASE } & 2.62 E+00\end{array}$

VOLUME OF LIQUID WASTE RELEASED (PRIOR TO DILUTION)
VOLUME OF DILUTION WATER USED DURING PERIOD 
INSTALLATION:MILLSTONE 1

EFFLUENT AND WASTE DISPOSAL ANNUAL REPORT FOR YEAR 1981 SOLID EFFLUENTS

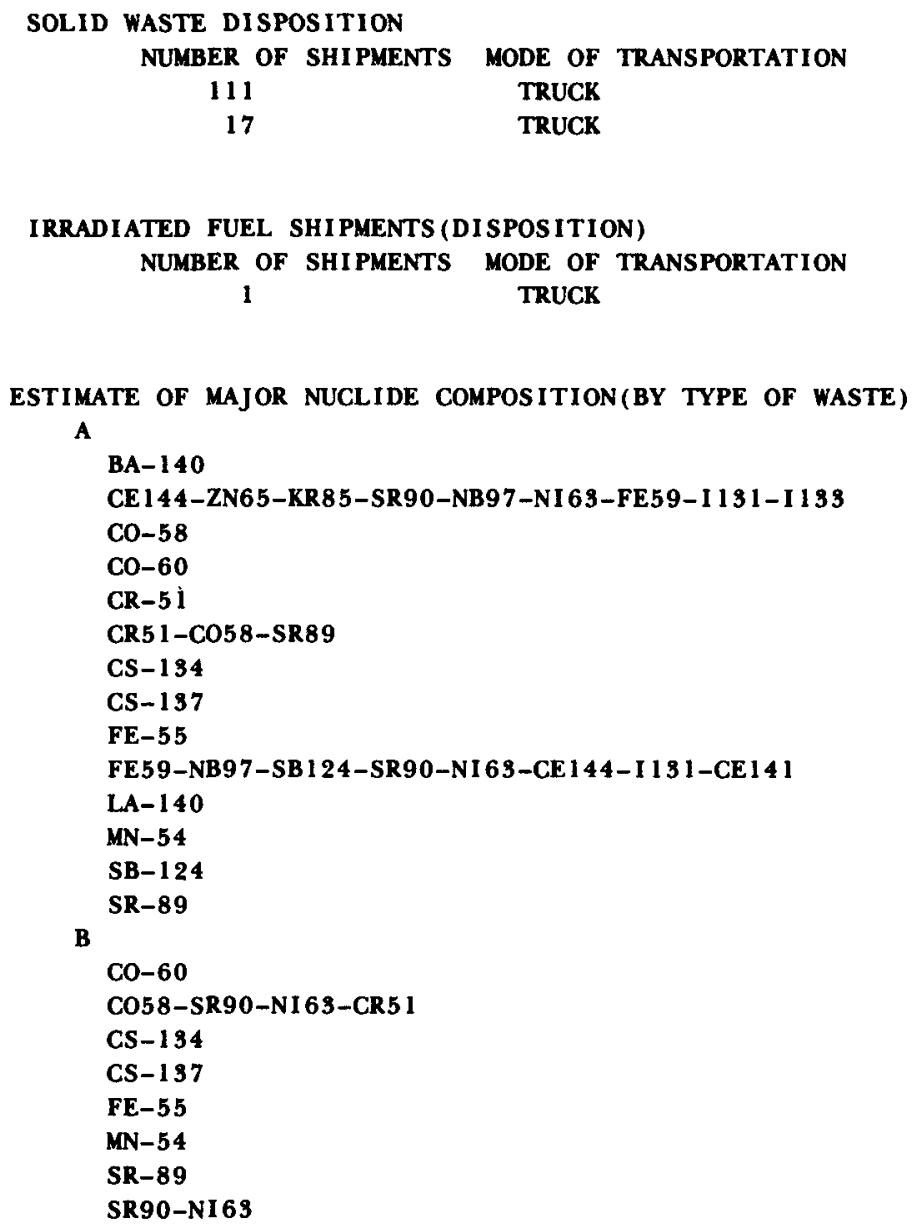

TYPE OF WASTE
A. SPENT RESINS, FILTER SLUDGES, EVAPORATOR BOTTOMS, ETC.
B. DRY COMPRESS IBLE WASTE, CONTAMINATED EQU I PMENT, ETC.
C. IRRADIATED COMPONENTS, CONTROL RODS, ETC.
D. OTHER

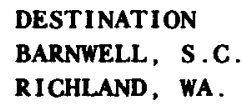

DESTINATION

PLEASANTON, CA

JAN-JUNE JULY-DEC

3. $00 \mathrm{E}+00$

1. $30 \mathrm{E}+00$

3. $00 \mathrm{E}+00$

4. $20 E+01 \quad 4.20 E+01$

3. $90 \mathrm{E}+00$

9.80E+00 4.10E+00

$1.80 E+01 \quad 1.20 E+01$

$9.00 E+00 \quad 1.60 E+01$

4. $70 \mathrm{E}+00$

1. 30E+00

$7.10 \mathrm{E}+00 \quad 8.80 \mathrm{E}+00$

7. 20E+00

3. $60 \mathrm{E}+00$

6. $20 E+01 \quad 4.00 E+01$

4. 70E+00

2.00E +00 3.40E+00

$7.00 \mathrm{E}+00 \quad 2.10 \mathrm{E}+01$

$7.00 \mathrm{E}+00 \quad 2.30 \mathrm{E}+01$

$1.60 \mathrm{E}+01 \quad 6.20 \mathrm{E}+00$

$1.30 E+00$ 3.30E+00

3. 10E+00

N/A=NOT APPLICABLE

$N / D=$ NOT DETECTED

N/R=NOT REPORTED

$\begin{array}{lc}\text { UNIT } & \text { YEAR TOTAL } \\ \text { M3 } & 2.74 E+02 \\ \text { CI } & 1.77 E+03 \\ \text { M3 } & 1.69 E+03 \\ \text { CI } & 5.79 E+01 \\ \text { M3 } & \\ \text { CI } & \\ \text { M3 } & \\ \text { CI } & \end{array}$ 
EFFLUENT AND WASTE DISPOSAL ANNUAL REPORT FOR YEAR 1981 AIRBORNE AND LIQUID EFFLUENTS

UNIT NUMBER: 2

TYPE : PWR

DOCKET NO. : 50-336

COOLING WATER SOURCE:LONG ISLAND SOUND

\section{AIRBORNE EFFLUENTS}

NUCLIDES RELEASED

H-3

AR-41

MN-54

CO-58

CO-60

BR-82

KR-83M

KR-85

KR-85M

KR-87

KR-88

RB-88

Y-88

SR-89

I-131

XE-191M

I-133

XE-133

XE-1 33M

I - 135

XE-195

XE-135M

CS-137

XE-137

CS-138

XE-138

LIQUID EFFLUENTS

NUCLIDES RELEASED

H-3

NA-24

CR-51

MN-54

CO-57

CO-58

FE-59

CO-60

RB-88

$S R-89$

SR-90

SR-92

NB-95

ZR-95

NB-97

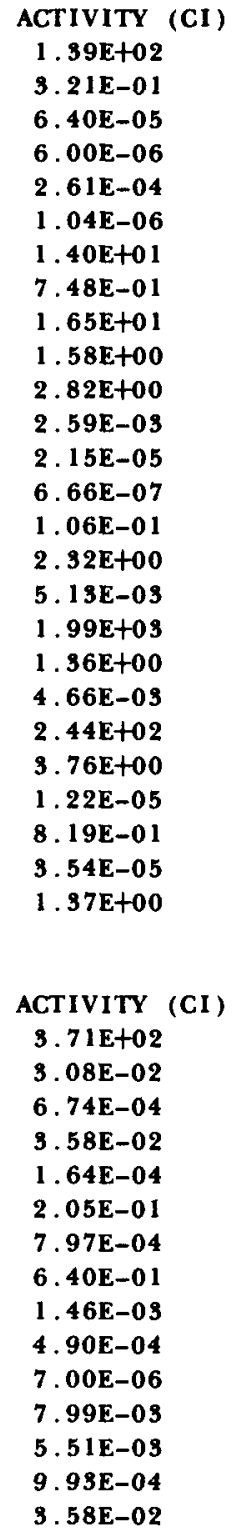

LICENSEE : NORTHEAST NUCLEAR ENERGY LICENSED POWER (MWT) : 2560.00 INITIAL CRITICALITY : $10 / 17 / 75$

N/A=NOT APPLICABLE $N / D=N O T$ DETECTED N/R=NOT REPORTED 
EFFLUENT AND WASTE DISPOSAL ANNUAL REPORT FOR YEAR 1981 AIRBORNE AND LIQUID EFFLUENTS

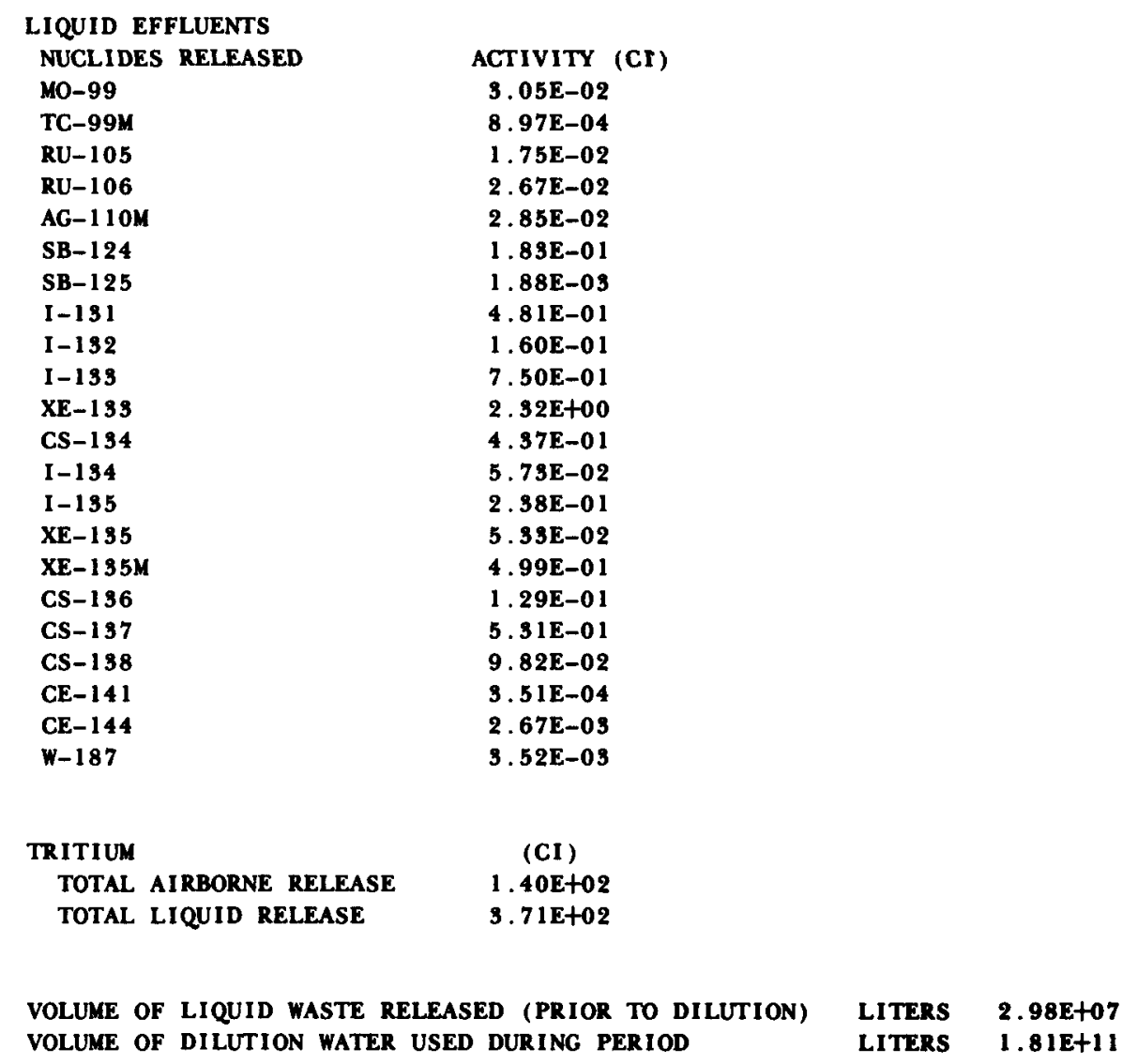

N/A $=$ NOT APPLICABLE

$N / D=N O T$ DETECTED

N/R=NOT REPORTED 
INSTALLATION : MILLSTONE 2

EFFLUENT AND WASTE DISPOSAL ANNUAL REPORT FOR YEAR 1981 SOLID EFFLUENTS

SOLID WASTE DISPOSITION

NUMBER OF SHIPMENTS MODE OF TRANSPORTATION 7

TRUCK

DESTINATION

BARNWELL, S.C.

IRRADI ATED FUEL SHI PMENTS (DI POS ITION)

NUMBER OF SHIPMENTS MODE OF TRANSPORTATION

DESTINATION

0

ESTIMATE OF MAJOR NUCLIDE COMPOS ITION(BY TYPE OF WASTE)

A

CO-58
CO-60
CR-5 1
CS-134
CS-197
FE-55
MN-54
NB-95
SR89-MN54-SR90-N163-FE55
ZR95-NB97-I131-CO57-SR89-SR90-NI63

TYPE OF WASTE

A. SPENT RESINS, FILTER SLUDGES, EVAPORATOR BOTTOMS, ETC.

B. DRY COMPRESS IBLE WASTE, CONTAMINATED EQUI PMENT, ETC.

JAN-JUNE JULY-DEC

. IRRADIATED COMPONENTS, CONTROL

RODS , ETC.

D. OTHER

$\begin{array}{lll}* & & 4.70 E+01 \\ * & 8.00 E+00 & 1.40 E+01 \\ * & & 5.60 E+00 \\ * & 2.80 E+01 & 1.30 E+01 \\ \% & 6.30 E+01 & 1.50 E+01 \\ * & & 1.00 E+00 \\ * & & 1.60 E+00 \\ \% & & 1.00 E+00 \\ \% & 1.00 E+00 & \\ * & & 1.80 E+00\end{array}$

UNIT YEAR TOTAL

M3 1.69E+01

CI $\quad 3.21 \mathrm{E}+02$

M3

CI

M3

CI

M3

CI

N/A=NOT APPLICABLE

$N / D=$ NOT DETECTED

N/R=NOT REPORTED 
EFFLUENT AND WASTE DISPOSAL ANNUAL REPORT FOR YEAR 1981 AIRBORNE AND LIQUID EFFLUENTS

UNIT NUMBER: 1

TYPE : BWR

DOCKET NO . : 50-263

COOLING WATER SOURCE : MISSISS IPPI RIVER

AIRBORNE EFFLUENTS

NUCLIDES RELEASED

CR-5 I

MN-54

CO-58

FE-59

C0-60

$\mathrm{ZN}-65$

KR-83M

KR-85

KR-85M

KR-87,

KR-88

KR -89

SR-89

KR-90

SR-90

NB-95

ZR-95

RU-103

I-131

$\mathrm{XE}-131 \mathrm{M}$

I-133

XE-133

$\mathrm{XE}-133 \mathrm{M}$

CS-134

I-135

XE-135

$\mathrm{XE}-135 \mathrm{M}$

CS-136

CS-137

XE- 137

$\mathrm{XE}-138$

$\mathrm{XE}-139$

BA-140

CE-141

CE-144

ND 147

LIQUID EFFLUENTS

NUCLIDES RELEASED

CO-60

I-131

I-139

CS-197
LICENSEE : NORTHERN STATES POWER

LICENSED POWER (MWT) :1670.00

INITIAL CRITICALITY : 12/10/70

N/A=NOT APPLICABLE

N/D $=$ NOT DETECTED

$N / R=N O T$ REPORTED
ACTIVITY (CI)
4. 24E-04
$2.56 \mathrm{E}-04$
3. $71 \mathrm{E}-05$
5. 28E-05
2. 14E-03
$8.12 \mathrm{E}-04$
7. 74E+00
$2.50 \mathrm{E}+02$
7. 27E+00
4. $12 \mathrm{E}+01$
$2.23 E+01$
7.70E+02
5. 17E-03
2.63E+0 1
1. 83E-04
5. $35 \mathrm{E}-05$
1.77E-05
3.33E-05
1. 55E-02
4. $75 \mathrm{E}+00$
$7.48 \mathrm{E}-02$
6.34E+02
$2.61 \mathrm{E}+00$
7. $18 \mathrm{E}-05$
3. 95E-02
3. 77E+01
5. 86E+01
$1.51 \mathrm{E}-06$
7. 80E-04
$1.01 E+03$
8. 13E+02
6. 30E+01
8. 85E-03
$6.75 \mathrm{E}-05$
1. 40E-05
1 . $17 \mathrm{E}-06$
ACTIVITY (CI)
1. 63E-06
2. 48E-07
1.03E-06
1. 99E-07 
EFFLUENT AND WASTE DISPOSAL ANNUAL REPORT FOR YEAR 1981 AIRBORNE AND LIQUID EFFLUENTS

$\begin{array}{lc}\text { TRITIUM } & \text { (CI) } \\ \text { TOTAL AIRBORNE RELEASE } & 1.10 \mathrm{E}+02 \\ \text { TOTAL LIQUID RELEASE } & 4.17 E-03\end{array}$
$\begin{array}{llll}\text { VOLUME OF LIQUID WASTE RELEASED (PRIOR TO DILUTION) } & \text { LITERS } & \text { 3.79E+02 } \\ \text { VOLUME OF DILUTION WATER USED DURING PERIOD } & \text { LITERS } & 0.00 E+00\end{array}$

N/A=NOT APPLICABLE

$N / D=N O T$ DETECTED

N/R=NOT REPORTED 
INSTALLATION : MONTICELLO

EFFLUENT AND WASTE DISPOSAL ANNUAL REPORT FOR YEAR 1981 SOLID EFFLUENTS

SOLID WASTE DISPOSITION

NUMBER OF SHIPMENTS MODE OF TRANSPORTATION

20 TRUCK

31 TRUCK

DESTINATION

BARNWELL, S.C.

RICHLAND, WA

I RRADI ATED FUEL SHI PMENTS (DISPOS ITION)

NUMBER OF SHIPMENTS MODE OF TRANSPORTATION

DESTINATION TRUCK

VALLECITOS, CA

ESTIMATE OF MAJOR NUGLIDE COMPOSITION(BY TYPE OF WASTE)

A
BA- 140
CE- 141
CO-58
CO-60
CR-5 I
CS-134
CS-137
I-13I
LA- 140
$\mathrm{MN}-54$
NB-95
SR-90
ZN-65
BA- 140
CE- 141
CO- 58
CO-60
CR-5I
CS-134
CS-137
FE-59
I-13I
LA- 140
MN-54
RU- 103
SR-90
ZN-65

B

N/A=NOT APPLICABLE

$N / D=N O T$ DETECTED

$N / R=$ NOT REPORTED

JAN-JUNE JULY-DEC

5. $90 \mathrm{E}+00$

5. $36 \mathrm{E}-01$

2. $61 \mathrm{E}-01$

1. $61 \mathrm{E}+01$

1. 19E+00

$6.81 E+00$

4. $12 \mathrm{E}+01$

5. 74E+00

6. 28E+00

$1.07 \mathrm{E}+00$

4. 32E-03

7.31E-03

1. 13E+0 1

$1.51 \mathrm{E}+01$

7. $90 \mathrm{E}-01$

3. 17E-01

8. 17E-02

1. 54E+00

1. $78 \mathrm{E}+00$

7. $78 \mathrm{E}+01$

5. $06 \mathrm{E}+01$

2. 26E+00

2. 83E+00

2. $28 \mathrm{E}+00$

7. 55E+00

2.37E+01

4. 88E-01

1. $06 \mathrm{E}+00$

8. 56E-01

2. 89E-0 1

$2.66 \mathrm{E}+00$

$7.89 E-01$

1. 45E-02

1. 42E-01

3. 47E+00

1. $27 \mathrm{E}+01$ 
INSTALLATION : MONTICELLO

EFFLUENT AND WASTE DISPOSAL ANNUAL REPORT FOR YEAR 1981 SOLID EFFLUENTS

TYPE OF WASTE

A. SPENT RESINS, FILTER SLUDGES, EVAPORATOR BOTTOMS, ETC.

B. DRY COMPRESS IBLE WASTE, CONTAMINATED EQUI PMENT, ETC

C. IRRADIATED COMPONENTS, CONTROL RODS, ETC.

D. OTHER
UNIT YEAR TOTAL

M3 $\quad 1.40 \mathrm{E}+02$

CI $\quad$ 4.23E+02

M3 4.14E+02

CI $1.94 \mathrm{E}+01$

M3

CI

M3

CI 
EFFLUENT AND WASTE DISPOSAL ANNUAL REPORT FOR YEAR 1981 AIRBORNE AND LIQUID EFFLUENTS

UNIT NUMBER: 1

TYPE: $\quad$ BWR

DOCKET NO . : 50-220

COOLING WATER SOURCE : LAKE ONTARIO

AIRBORNE EFFLUENTS

NUCLI DES RELEASED

CR-51

$\mathrm{MN}-54$

CO-58

CO-60

KR-85M

KR-88

SR-89

SR-90

I-131

I-133

XE- 133

I-135

XE-135

XE-135M

CS- 137

$\mathrm{XE}-138$

BA-LA-140

LIQUID EFFLUENTS

NUCLIDES RELEASED

MN-54

CO-58

CO-60

SR-89

SR-90

$\mathrm{XE}-133$

CS-134

XE- 135

CS-137

TRITIUM

TOTAL AIRBORNE RELEASE TOTAL LIQUID RELEASE
ACTIVITY (CI)

1.94E-04

$1.22 \mathrm{E}+00$

4. 65E-05

6. 10E-03

5.37E+01

6. $89 \mathrm{E}+01$

$<5.60 \mathrm{E}-04$

$<6.87 \mathrm{E}-05$

$6.44 \mathrm{E}-03$

5. 62E-02

$7.49 \mathrm{E}+01$

2.92E-01

1. $58 \mathrm{E}+02$

$2.36 \mathrm{E}+02$

1.35E-03

$1.99 \mathrm{E}+01$

3. 70E-05

ACTIVITY (CI)

9. 32E-02

$2.58 \mathrm{E}-02$

1. $16 E+00$

3.06E-04

2.10E-03

$<1.91 \mathrm{E}-02$

5. 40E-01

$<2.89 \mathrm{E}-01$

3. $53 \mathrm{E}+00$

(CI)

$6.34 \mathrm{E}+01$

$5.05 \mathrm{E}+00$
LICENSEE : NI AGARA MOHAWK POWER

LICENSED POWER (MWT) $: 1850.00$

INITIAL CRITICALITY : 09/05/69

VOLUME OF LIQUID WASTE RELEASED (PRIOR TO DILUTION) LITERS 4.32E+05 VOLUME OF DILUTION WATER USED DURING PERIOD

LITERS $\quad 7.81 \mathrm{E}+08$

N/A=NOT APPLICABLE *

$N / D=N O T$ DETECTED

N/R=NOT REPORTED 
INSTALLATION : NINE MILE POINT

EFFLUENT AND WASTE DISPOSAL ANNUAL REPORT FOR YEAR 1981 SOLID EFFLUENTS

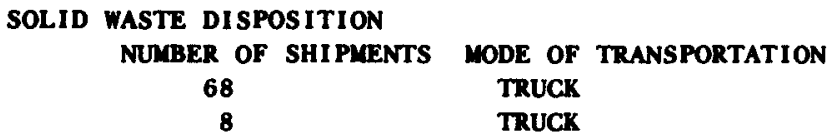

IRRADIATED FUEL SHI PUENTS (DISPOSITION)

NUMBER OF SHIPMENTS MODE OF TRANSPORTATION

ESTIMATE OF MAJOR NUCLIDE COMPOSITION(BY TYPE OF WASTE)

$\mathbf{A}$

$$
\begin{aligned}
& \text { CO-58 } \\
& \text { CO-60 } \\
& \text { CS-134 } \\
& \text { CS-137 } \\
& M N-54 \\
& \text { CO-58 } \\
& \text { CO-60 } \\
& \text { CS-134 } \\
& \text { CS-137 } \\
& M N-54
\end{aligned}
$$

B

TYPE OF WASTE
A. SPENT RESINS, FILTER SLUDGES, EVAPORATOR BOTTOMS, ETC
B. DRY COMPRESSIBLE WASTE, CONTAMINATED EQUI PMENT, ETC.
C. IRRADIATED COMPONENTS, CONTROL RODS, ETC.
D. OTHER

DESTINATION BARNWELL SC HANFORD WA

DESTINATION

JAN-JUNE JULY-DEC
$5.00 \mathrm{E}+01$
$1.00 E+01$
5. $06 \mathrm{E}+01$
$4.00 E+01$
6. 20E+00
3. $86 \mathrm{E}+01$
4. $50 \mathrm{E}+00$
$5.00 E+01$
$1.00 \mathrm{E}-01$
$1.00 E+01$
$5.60 \mathrm{E}+01$
4. 00E+01
$6.20 \mathrm{E}+00$
3. 86E+01
4. 50E+00

1.00E-01

UNIT YEAR TOTAL

M3 $2.37 \mathrm{E}+02$

CI $1.71 \mathrm{E}+03$

M3 2.94E+02

CI $1.14 \mathrm{E}+01$

M3 $0.00 \mathrm{E}+00$

CI

M3

CI

N/A=NOT APPLICABLE

N/D=NOT DETECTED

N/R=NOT REPORTED 
EFFLUENT AND WASTE DISPOSAL ANNUAL REPORT FOR YEAR 1981 AIRBORNE AND LIQUID EFFLUENTS

UNIT NUMBER : 1

TYPE: PWR

DOCKET NO. : 50-338

COOLING WATER SOURCE : LAKE ANNA

UNIT NUMBER : 2

TYPE : PWR

DOCKET NO. : 50-339

COOLING WATER SOURCE: LAKE ANNA
LICENSEE : VIRGINIA ELECTRIC \&POWER

LICENSED POWER (MWT) : 2775.00

INITIAL CRITICALITY : $04 / 05 / 78$

LICENSEE : VIRGINIA ELECTRIC \&POWER

LICENSED POWER (MWT) : 2775.00

INITIAL CRITICALITY: 06/12/80
A IRBORNE EFFLUENTS

NUCLIDES RELEASED

MN-54

CO-58

$\mathrm{CO}-60$

KR-85M

$\mathrm{KR}-88$

SR-89

SR-90

I-131

X'E-13IM

I-133

$\mathrm{XE}-133$

$\mathrm{XE}-139 \mathrm{M}$

CS -134

$1-195$

LIQUID EFFLUENTS

NUCLIDES RELEASED

NA-24

CR-5 1

MN -54

CO-58

FE-59

CO-60

ZR-NB-95

TC-99M

AG-1 $10 \mathrm{M}$

SB-124

I-131

TE- $131 \mathrm{M}$

I-133

XE-133

XE- $133 \mathrm{M}$

CS-134

XE-135

CS-137

BA-LA- 140
ACTIVITY (CI)

1.03E-03

5.02E-03

6 . 19E-03

2.82E-03

2. 26E-03

1. 48E-05

3. 34E-07

4. $66 \mathrm{E}-01$

$5.76 \mathrm{E}+00$

7.03E-02

5. 20E+03

2. 23E+01

1.17E-03

3.06E-03

8.03E+01

3. 94E-03

1. 98E-03

ACTIVITY (CI)

2.62E-02

3. $62 \mathrm{E}-03$

3. 15E-03

1. 93E-01

1. 23E-03

1. 34E-01

1. 55E-03

5. 59E-04

1. 13E-02

3. 30E-03

9. 75E-02

1. 05E-04

5. 71 E-03

4. $28 \mathrm{E}+00$

2. 10E-02

7. 22E-02

4. $51 \mathrm{E}-02$

1. 22E-01

2. 72E-04

N/A =NOT APPLICABLE

$N / D=N O T$ DETECTED

N/R=NOT REPORTED 
EFFLUENT AND WASTE DISPOSAL ANNUAL REPORT FOR YEAR 1981 AIRBORNE AND LIQUID EFFLUENTS
TR ITIUM
TOTAL AIRBORNE RELEASE
TOTAL LIQUID RELEASE
(CI)
3. $14 E+01$
1. 28E+03

VOLUME OF LIQUID WASTE RELEASED (PRIOR TO DILUTION)

VOLUME OF DILUTION WATER USED DURING PERIOD

LITERS

LITERS

$1.85 \mathrm{E}+08$

$1.70 E+12$

N/A=NOT APPLICABLE

N/D $=$ NOT DETECTED

N/R=NOT REPORTED 
INSTALLATION: NORTH ANNA

EFFLUENT AND WASTE DISPOSAL ANNUAL REPORT FOR YEAR 1981 SOLID EFFLUENTS

SOLID WASTE DISPOSITION NUMBER OF SHIPMENTS MODE OF TRANSPORTATION DESTINATION 27

IRRADIATED FUEL SHIPMENTS (DISPOSITION) NUMBER OF SHIPMENTS MODE OF TRANSPORTATION

DESTINATION 0

ESTIMATE OF MAJOR NUCLIDE COMPOSITION(BY TYPE OF WASTE) A

CO-57

C0-58

CO-60

CS-134

CS -137

MN-54

B

Co-58

C0-60

CS-134

CS-137

MN-54

TYPE OF WASTE

A. SPENT RESINS, FILTER SLUDGES, EVAPORATOR BOTTOMS, ETC.

B. DRY COMPRESS IBLE WASTE, CONTAMINATED EQUI PMENT, ETC.

JAN-JUNE JULY-DEC

IRRADIATED COMPONENTS, CONTROL RODS , ETC.

D. OTHER

2.80E+01

$3.80 E+01$

1. $20 \mathrm{E}+01$

1 . 50E+01

$6.90 \mathrm{E}+00$

1. 50E-0 1

$1.52 E+00$

5.57E+01

1. 26E+01

3. 74E+01

2. 68E+00

2.80E+01

$3.80 E+01$

5. 39E+01

1. 20E+01

$9.88 \mathrm{E}+00$

1. $50 \mathrm{E}+01$

1 . $18 \mathrm{E}+01$

$6.90 \mathrm{E}+00$

2. 24E+01

2.08E+00

N/A =NOT APPLICABLE

N/D $=$ NOT DETECTED

N/R=NOT REPORTED

CI 
EFFLUENT AND WASTE DISPOSAL ANNUAL REPORT FOR YEAR 1981 AIRBORNE AND LIQUID EFFLUENTS

UNIT NUMBER: 1

TYPE: $\quad$ PWR

DOCKET NO . : 50-269

COOLING WATER SOURCE:KEOWEE LAKE UNIT NUMBER : 2

TYPE: $\quad$ PWR

DOCKET NO. : 50-270

. COOLING WATER SOURCE : KEOWEE LAKE

UNIT NUMBER: 3

TYPE: PWR

DOCKET NO. : 50-287

COOLING WATER SOURCE : KEOWEE LAKE

\section{AIRBORNE EFFLUENTS}

NUCLIDES RELEASED

AR-41

CR-5 1

MN-54

CO-58

FE-59

CO-60

$\mathrm{ZN}-65$

KR-85

KR-85M

KR-87

KR-88

KR-89

SR-89

SR-90

NB-95

ZR-95

MO-99

RU- 103

RU- 106

AG-1 10M

SB-125

I-131

$\mathrm{XE}-131 \mathrm{M}$

I-1 93

XE- 133

XE-133M

CS-134

I-135

$\mathrm{XE}-135$

XE-135M

CS-136

CS-137

BA-140

CE-144
LICENSEE : DUKE POWER

LICENSED POWER (MWT) : 2568.00 INITIAL CRITICALITY :04/19/73

LICENSEE : DUKE POWER

LICENSED POWER (MWT): 2568.00 INITIAL CRITICALITY : $11 / 11 / 73$

LICENSEE : DUKE POWER

LICENSED POWER (MWT) : 2568.00 INITIAL CRITICALITY :09/05/74

N/A=NOT APPLICABLE

N/D $\approx$ NOT DETECTED

N/R=NOT REPORTED

ACTIVITY (CI)
$1.14 E+01$
$1.40 E-03$
$1.45 E-04$
$6.74 E-03$
$1.18 E-04$
$1.58 E-03$
$1.25 E-04$
$3.02 E+02$
$5.85 E+00$
$1.06 E+01$
$2.58 E+00$
$4.84 E+01$
$2.98 E-08$
$2.97 E-09$
$5.04 E-05$
$5.26 E-04$
$9.19 E-04$
$9.68 E-05$
$1.10 E-03$
$8.84 E-04$
$2.16 E-04$
$2.50 E-01$
$1.85 E+02$
$2.78 E-02$
$1.54 E+04$
$1.18 E+02$
$5.39 E-03$
$1.99 E-02$
$2.07 E+02$
$6.77 E+00$
$2.67 E-04$
$6.15 E-03$
$5.24 E-04$
$7.62 E-04$


EFFLUENT AND WASTE DISPOSAL ANNUAL REPORT FOR YEAR 1981 AIRBORNE AND LIQUID EFFLUENTS

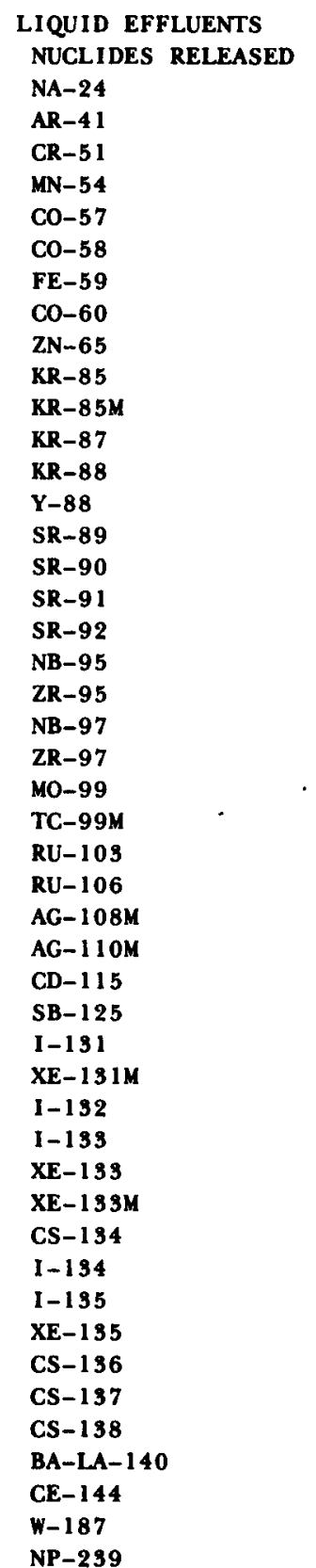

N/A=NOT APPLICABLE N/D=NOT DETECTED N/R=NOT REPORTED

$$
\begin{aligned}
& \text { ACTIVITY (CI) } \\
& 2.29 E-04 \\
& 8.70 E-05 \\
& 3.82 E-02 \\
& 1.05 E-02 \\
& 1.56 E-04 \\
& 1.98 E-01 \\
& 1.13 E-04 \\
& 2.83 E-02 \\
& 2.32 E-05 \\
& 1.15 E+00 \\
& 5.53 E-04 \\
& 8.87 E-06 \\
& 2.95 E-04 \\
& 2.12 E-05 \\
& 1.54 E-02 \\
& 1.74 E-03 \\
& 3.56 E-03 \\
& 7.16 E-04 \\
& 5.11 E-04 \\
& 4.05 E-05 \\
& 1.44 E-02 \\
& 3.73 E-05 \\
& 2.41 E-02 \\
& 3.33 E-05 \\
& 1.63 E-05 \\
& 3.33 E-02 \\
& 2.50 E-06 \\
& 3.08 E-03 \\
& 3.14 E-03 \\
& 7.79 E-03 \\
& 1.16 E-01 \\
& 9.93 E-02 \\
& 1.70 E-04 \\
& 1.91 E-03 \\
& 5.37 E+00 \\
& 5.39 E-02 \\
& 4.85 E-01 \\
& 1.52 E-03 \\
& 2.23 E-03 \\
& 1.67 E-01 \\
& 1.50 E-02 \\
& 6.72 E-01 \\
& 1.97 E-03 \\
& 1.75 E-02 \\
& 2.61 E-02 \\
& 1.37 E-02 \\
& 9.50 E-03 \\
&
\end{aligned}
$$


EFFLUENT AND WASTE DISPOSAL ANNUAL REPORT FOR YEAR 1981 AIRBORNE AND LIQUID EFFLUENTS

TRITIUM

TOTAL AIRBORNE RELEASE

TOTAL LIQUID RELEASE
(CI)

5.77E+01

$5.07 E+02$

VOLUME OF LIQUID WASTE RELEASED (PRIOR TO DILUTION)

LITERS

VOLUME OF DILUTION WATER USED DURING PERIOD

LITERS

1. $56 \mathrm{E}+08$

6. $20 \mathrm{E}+11$

N/A=NOT APPLICABLE

N/D=NOT DETECTED

N/R = NOT REPORTED 
INSTALLATION : OCONEE

EFFLUENT AND WASTE DISPOSAL ANNUAL REPORT FOR YEAR 1981 SOLID EFFLUENTS

SOLID WASTE DISPOSITION

NUMBER OF SHIPMENTS MODE OF TRANSPORTATION 193 TRUCK

DESTINATION

BARNWELL, SC

TYPE OF WASTE

A. SPENT RESINS, FILTER SLUDGES, EVAPORATOR BOTTOMS, ETC.

UNIT YEAR TOTAL'

M3 $2.48 E+03$

CI $1.12 E+04$

B. DRY COMPRESSIBLE WASTE, CONTAMINATED

MS

EQU I PMENT, ETC.

CI

C. IRRADI ATED COMPONENTS, CONTROL RODS, ETC.

D. OTHER 
EFFLUENT AND WASTE DISPOSAL ANNUAL REPORT FOR YEAR 1981 AIRBORNE AND LIQUID EFFLUENTS

\begin{abstract}
UNIT NUNBER : I
TYPE: $\quad$ BWR
\end{abstract}

DOCKET NO. : 50-219

COOLING WATER SOURCE : BARNEGAT BAY

\section{AIRBORNE EFFLUENTS}

NUCLIDES RELEASED

CR-51

MN-54

C0-57

C0-58

C0-60

KR-85M

KR-87

KR-88

KR-89

SR-89

SR-90

SR-9 1

NB-95

ZR-95

M0-99

TC-99M

I-131

1-133

XE-133

XE-133M

I -135

XE-135

$\mathrm{XE}-135 \mathrm{M}$

CS-137

$\mathrm{XE}-137$

$\mathrm{XE}-138$

BA-140

LA-140

CE-141

CE-143

CE-144

PA-233

NP-239

LIQUID EFFLUENTS

NUCLIDES RELEASED

CR-51

$\mathrm{MN}-54$

CO-57

CO-58

CO-60

SR-89

SR-90

SR-91

N/A=NOT APPLICABLE N/D=NOT DETECTED N/R=NOT REPORTED
LICENSEE: JERSEY CENTRAL POWER \& LIGHT LICENSED POWER (MWT) : 1930.00 INITIAL CRITICALITY : 05/03/69

\section{ACTIVITY (CI)}

2.94E-03

1.79E-02

2.40E-04

$1.49 \mathrm{E}-03$

4.50E-03

$1.92 E+03$

$6.99 \mathrm{E}+03$

$5.85 \mathrm{E}+03$

$1.67 \mathrm{E}-01$

4. 72E-01

6.84E-03

2. 98E+00

5. 11E-03

2.12E-04

1. $13 \mathrm{E}-02$

3.03E-01

9. 15E-01

3. 38E+00

$1.03 E+03$

3. 32E+01

$4.83 E+00$

1. $20 E+04$

4. 14E+03

4. $11 \mathrm{E}-02$

3. $68 E+03$

1. $53 \mathrm{E}+04$

7. 35E-01

5. 37E-01

7. 35E-04

1. 40E-03

3. $41 \mathrm{E}-02$

9. 89E-05

1. 20E-02

ACTIVITY (CI)

3. 95E-02

8. 66E-03

8. 40E-04

6. 13E-05

7. 97E-02

6. 68E-03

5.63E-04

8. 14E-04 
EFFLUENT AND WASTE DISPOSAL ANNUAL REPORT FOR YEAR 1981 AIRBORNE AND LIQUID EFFLUENTS

\begin{tabular}{|c|c|}
\hline \multicolumn{2}{|l|}{ LIQUID EFFLUENTS } \\
\hline NUCLIDES RELEASED & ACTIVITY (CI \\
\hline Mo-99 & $9.08 \mathrm{E}-05$ \\
\hline TC-99M & $7.18 \mathrm{E}-03$ \\
\hline RU-103 & $1.26 \mathrm{E}-04$ \\
\hline SB-124 & 1. $65 E-03$ \\
\hline SB-1 25 & 3. $48 \mathrm{E}-04$ \\
\hline $1-191$ & 3. 30E-03 \\
\hline $1-193$ & $8.84 E-03$ \\
\hline$X E-193$ & $1.04 E-01$ \\
\hline XE-1 33M & 3.87E-04 \\
\hline CS-1 34 & $3.01 \mathrm{E}-03$ \\
\hline$I-135$ & $5.16 \mathrm{E}-02$ \\
\hline$X E-195$ & $2.89 \mathrm{E}-01$ \\
\hline CS-137 & 1. 74E-02 \\
\hline$B A-140$ & 4. 36E-03 \\
\hline LA- 140 & $6.32 \mathrm{E}-03$ \\
\hline CE- 141 & $6.16 E-04$ \\
\hline CE- 143 & $1.44 E-04$ \\
\hline CE-144 & $6.18 \mathrm{E}-03$ \\
\hline PA-233 & $4.04 \mathrm{E}-05$ \\
\hline NP-239 & $4.92 E-04$ \\
\hline TRITIUM & (CI) \\
\hline TOTAL AIRBORNE RELEASE & 3. 22E+00 \\
\hline TOTAL LIQUID RELEASE & $2.67 \mathrm{E}+01$ \\
\hline
\end{tabular}

VOLUME OF LIQUID WASTE RELEASED (PRIOR TO DILUTION) LITERS 8.42E+06 VOLUME OF DILUTION WATER USED DURING PERIOD 
EFFLUENT AND WASTE DISPOSAL ANNUAL REPORT FOR YEAR 1981 SOLID EFFLUENTS

SOLID WASTE DISPOSITION NUMBER OF SHIPMENTS 139
1
15 MODE OF TRANSPORTATION MOTOR VEHICLE MOTOR VEHICLE MOTOR VEHICLE

IRRADIATED FUEL SHI PMENTS (DISPOSITION) NUMBER OF SHIPMENTS MODE OF TRANSPORTATION 0

MOTOR VEHICLE

ESTIMATE OF MAJOR NUCLIDE COMPOSITION(BY TYPE OF WASTE)

A

BA- 140

$\mathrm{CO}-60$

CS-137

I-133

LA-140

$M N-54$

SR-89

B

CO-58

CO-60

CS-137

I-133

MN-54

SR-89

TYPE OF WASTE

A. SPENT RESINS, FILTER SLUDGES, EVAPORATOR BOTTOMS, ETC.

B. DRY COMPRESS IBLE WASTE, CONTAMINATED EQUI PMENT, ETC.

C. IRRADIATED COMPONENTS, CONTROL RODS, ETC

D. OTHER
DESTINATION

BARNWELL, S.C.

BEATTY, NV

RICHLAND, WA

DESTINATION

COLUMBUS, OH

JAN-JUNE JULY-DEC

$\begin{array}{ll}5.40 \mathrm{E}+01 & \begin{array}{l}4.00 \mathrm{E}+00 \\ 8.20 \mathrm{E}+01\end{array} \\ \text { 8.20E }+00 & \\ 4.30 \mathrm{E}+00 & 3.60 \mathrm{E}+00 \\ 7.90 \mathrm{E}+00 & 8.40 \mathrm{E}+00 \\ 1.30 \mathrm{E}+01 & 2.90 \mathrm{E}+01 \\ & \\ 5.50 \mathrm{E}+00 & \\ 2.80 \mathrm{E}+01 & 6.30 \mathrm{E}+01 \\ 1.00 \mathrm{E}+01 & 3.70 \mathrm{E}+00 \\ & 2.10 \mathrm{E}+00 \\ 3.40 \mathrm{E}+01 & 1.50 \mathrm{E}+01 \\ 7.20 \mathrm{E}+00 & 4.50 \mathrm{E}+00\end{array}$

UNIT YEAR TOTAI

M3 5.78E+02

CI $2.83 E+02$

M3 $1.20 \mathrm{E}+03$

CI $1.38 \mathrm{E}+02$

M3 $0.00 \mathrm{E}+00$

CI $\quad 0.00 E+00$

M3

CI 
EFFLUENT AND WASTE DISPOSAL ANNUAL REPORT FOR YEAR 1981 AIRBORNE AND LIQUID EFFLUENTS

UNIT NUMBER : 1

TYPE: $\quad$ PWR

DOCKET NO. : 50-255

COOLING WATER SOURCE: LAKE MICHIGAN

AIRBORNE EFFLUENTS

NUCLIDES RELEASED

CR-5 1

MN-54

C0-58

$\mathrm{CO}-60$

SE-75

KR-85

KR-85M

KR-87

KR-88

MO-99

RU-103

I-131

XE-131M

I-132

I-133

XE-133

$\mathrm{XE}-133 \mathrm{M}$

CS-134

I- 135

XE-195

$\mathrm{XE}-135 \mathrm{M}$

CS-137

BA-140

LA-140

UNIDENT I F IED

LIQUID EFFLUENTS

NUCLIDES RELEASED

CR-51

MN -54

CO-57

CO-58

CO-60

NB-95

ZR-95

RU- 103

SB-1 25

1-131

$\mathrm{XE}-133$

CS-134

CS-137

UNIDENT I F IED
LICENSEE : CONSUMERS POWER

LICENSED POWER (MWT) $: 2530.00$

INITIAL CRITICALITY:05/24/71

$$
\begin{aligned}
& \text { ACTIVITY (CI) } \\
& 9.67 E-05 \\
& 7.58 E-05 \\
& 3.15 E-04 \\
& 2.40 E-04 \\
& 1.51 E-05 \\
& 5.21 E+00 \\
& 1.55 E-01 \\
& 2.16 E-01 \\
& 3.29 E-01 \\
& 4.07 E-07 \\
& 1.17 E-06 \\
& 4.05 E-02 \\
& 2.44 E+00 \\
& 1.66 E-03 \\
& 1.15 E-02 \\
& 2.99 E+03 \\
& 2.60 E-01 \\
& 2.63 E-05 \\
& 3.44 E-03 \\
& 1.08 E+00 \\
& 5.02 E-01 \\
& 2.50 E-04 \\
& 1.13 E-06 \\
& 3.00 E-06 \\
& 5.79 E-05
\end{aligned}
$$

ACTIVITY (CI)

5. 12E-04

3. $60 \mathrm{E}-03$

4.04E-06

$1.06 \mathrm{E}-02$

$6.85 \mathrm{E}-03$

1. 59E-04

6. $71 \mathrm{E}-05$

3.87E-05

2. 22E-04

8. 16E-05

2. 45E-03

2. 55E-03

6. 25E-03

2.08E-03

N/A=NOT APPLICABLE

$N / D=N O T$ DETECTED

N/R=NOT REPORTED 
EFFLUENT AND WASTE DISPOSAL ANNUAL REPORT FOR YEAR 1981 AIRBORNE AND LIQUID EFFLUENTS
TRITIUM
TOTAL AIRBORNE RELEASE
TOTAL LIQUID RELEASE
(CI)
6. $42 E+00$
2. 78E+02

VOLUME OF LIQUID WASTE RELEASED (PRIOR TO DILUTION)
VOLUME OF DILUTION WATER USED DURING PERIOD

N/A=NOT APPLICABLE

N/D $=$ NOT DETECTED

N/R=NOT REPORTED 
INSTALLATION : PALI SADES

EFFLUENT AND WASTE DISPOSAL ANNUAL REPORT FOR YEAR 1981 SOLID EFFLUENTS

SOLID WASTE DISPOSITION NUMBER OF SHIPMENTS

MODE OF TRANSPORTATION TRUCK

34

TRUCK

\section{DESTINATION}

BARNWELL, S.C.

RICHLAND, WA

IRRADI ATED FUEL SHI PMENTS (DI SPOS ITION)

NUMBER OF SHIPMENTS MODE OF TRANSPORTATION

DESTINATION

0

TYPE OF WASTE

A. SPENT RESINS, FILTER SLUDGES, EVAPORATOR

UNIT YEAR TOTAL

BOTTOMS, ETC.

B. DRY COMPRESS IBLE WASTE, CONTAMINATED

MS $1.33 E+02$

EQUI PMENT, ETC

C. IRRADIATED COMPONENTS, CONTROL RODS, ETC.

CI $1.02 E+02$

M3 7.20E+02

CI $2.29 \mathrm{E}+01$

M3 3.70E-01

CI $1.56 \mathrm{E}+04$

D. OTHER 
EFFLUENT AND WASTE DISPOSAL ANNUAL REPORT FOR YEAR 1981 AIRBORNE AND LIQUID EFFLUENTS

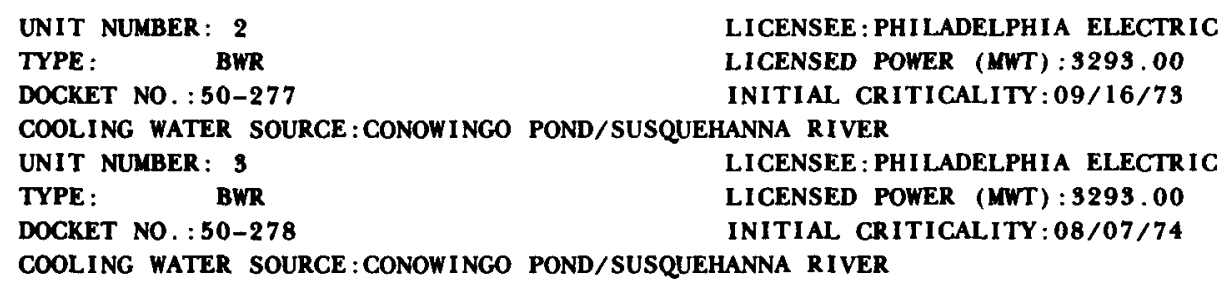

\section{LIQUID EFFLUENTS}

NUCLIDES RELEASED

H-3

NA-24

P-32

CR-5 1

MN-54

ACTIVITY (CI)
2. 49E+01
6. 73E-01
1. 74E-02
2.33E-02
2.57E-03

N/A=NOT APPLICABLE $N / D=N O T$ DETECTED N/R=NOT REPORTED 
EFFLUENT AND WASTE DISPOSAL ANNUAL REPORT FOR YEAR 1981 AIRBORNE AND LIQUID EFFLUENTS

\begin{tabular}{lc} 
LIQUID EFFLUENTS & \\
NUCLIDES RELEASED & ACTIVITY (C $)$ \\
FE-55 & $2.44 E-03$ \\
CO-58 & $9.76 E-03$ \\
CO-60 & $1.30 E-01$ \\
NI-63 & $3.99 E-03$ \\
ZN-65 & $2.90 E-01$ \\
SR-89 & $1.48 E-03$ \\
SR-90 & $1.59 E-03$ \\
SR-91 & $1.46 E-03$ \\
Y-91M & $2.68 E-03$ \\
SR-92 & $1.05 E-03$ \\
NB-95 & $4.98 E-04$ \\
TC-99M & $7.06 E-03$ \\
AG-110M & $1.84 E-03$ \\
I-131 & $5.39 E-02$ \\
XE-131M & $4.14 E-04$ \\
I-132 & $2.76 E-03$ \\
TE-132 & $8.04 E-03$ \\
I-133 & $8.12 E-02$ \\
XE-133 & $5.31 E-01$ \\
XE-133M & $1.25 E-03$ \\
CS-134 & $9.92 E-02$ \\
I-135 & $2.28 E-02$ \\
XE-135 & $2.34 E-01$ \\
XE-135M & $3.46 E-02$ \\
CS-137 & $1.70 E-01$ \\
BA-140 & $3.71 E-04$ \\
LA-140 & $4.73 E-03$ \\
NP-239 & $1.27 E-02$ \\
& \\
TRITIUM & \\
TOTAL AIRBORNE RELEASE & $2.85 E+01$ \\
TOTAL LIQUID RELEASE & $3.68 E+01$ \\
& \\
\hline & \\
&
\end{tabular}

VOLUME OF LIQUID WASTE RELEASED (PRIOR TO DILUTION) LITERS 2.69E+07 VOLUME OF DILUTION WATER USED DURING PERIOD

LITERS $\quad 1.66 \mathrm{E}+11$

N/A $=$ NOT APPLICABLE

$N / D=N O T$ DETECTED

N/R=NOT REPORTED 
INSTALLATION : PEACH BOTTOM

EFFLUENT AND WASTE DISPOSAL ANNUAL REPORT FOR YEAR 1981 SOLID EFFLUENTS

SOLID WASTE DISPOSITION NUMBER OF SHIPMENTS 303

MODE OF TRANSPORTATION TRUCK

32 TRUCK

DESTINATION

BARNWELL SC

RICHLAND WA

TYPE OF WASTE

A. SPENT RESINS, FILTER SLUDGES, EVAPORATOR BOTTOMS, ETC.

UNIT YEAR TOTAL

M3 2.34E+03

CI $5.33 E+03$

B. DRY COMPRESS IBLE WASTE, CONTAMINATED EQUI PMENT , ETC

M3

CI

. IRRADIATED COMPONENTS, CONTROL

M3 RODS, ETC.

D. OTHER

CI

M3

CI

N/A=NOT APPLICABLE

$N / D=N O T$ DETECTED

N/R=NOT REPORTED 
EFFLUENT AND WASTE DISPOSAL ANNUAL REPORT FOR YEAR 1981 AIRBORNE AND LIQUID EFFLUENTS

UNIT NUMBER: 1

TYPE: $\quad$ BWR

DOCKET NO. : 50-293

COOLING WATER SOURCE: CAPE COD BAY
LICENSEE : BOSTON EDISON

LICENSED POWER (MWT) : 1998.00

INITIAL CRITICALITY:06/16/72

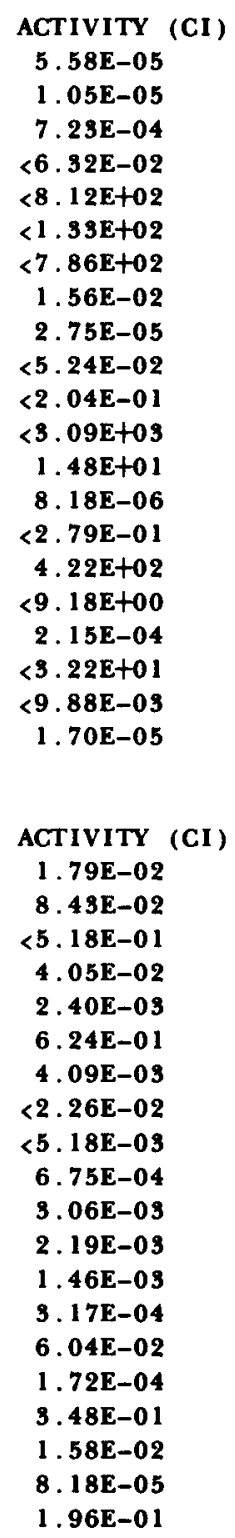

N/A=NOT APPLICABLE $N / D=N O T$ DETECTED N/R=NOT REPORTED 
EFFLUENT AND WASTE DISPOSAL ANNUAL REPORT FOR YEAR 1981 AIRBORNE AND LIQUID EFFLUENTS

LIQUID EFFLUENTS
NUCLIDES RELEAS

TR ITIUM

TOTAL AIRBORNE RELEASE

TOTAL LIQUID RELEASE
ACTIVITY (CI)

(CI)

7. $69 \mathrm{E}+01$

3. $41 \mathrm{E}+01$

VOLUME OF LIQUID WASTE RELEASED (PRIOR TO DILUTION)

VOLUME OF DILUTION WATER USED DURING PERIOD

LITERS 4.68E+06

LITERS $2.15 E+10$

N/A=NOT APPLICABLE

$N / D=$ NOT DETECTED

N/R=NOT REPORTED 
INSTALLATION : PILGR IM

EFFLUENT AND WASTE DISPOSAL ANNUAL REPORT FOR YEAR 1981 SOLID EFFLUENTS

SOLID WASTE DISPOSITION NUMBER OF SHIPMENTS MODE OF TRANSPORTATION 78 TRACTOR TRAILER

IRRADIATED FUEL SHI PMENTS (DISPOS ITION) NUMBER OF SHIPMENTS MODE OF TRANSPORTATION 0

ESTIMATE OF MAJOR NUCLIDE COMPOSITION(BY TYPE OF WASTE)

A

BA- 140

BA-LA- 140

CE-141

CO-58

CO-60

CR-51

CS-1 184

CS- 137

FE-55

FE-59

I-13I

I-133

LA-140

MN-54

MO-99

MO-99/TC-99M

RU- 103

SR-89

SR-90

TC-99M

$\mathrm{XE}-131 \mathrm{M}$

$\mathrm{ZN}-65$

B

BA-140

CO-58

CO-60

CR-51

CS-134

CS-137

FE-55

FE-59

I-131

LA-140

MN-54

NB-97

SB-1 22

SR-89
DESTINATION

BARNWELL, S.C.

DESTINATION

JAN-JUNE JULY-DEC

4. 20E-0 I

$2.00 \mathrm{E}-02 \quad 8.60 \mathrm{E}-02$

$1.47 \mathrm{E}+00 \quad 3.78 \mathrm{E}+00$

4. $16 \mathrm{E}+01$ 3. $65 \mathrm{E}+01$

$1.01 \mathrm{E}+00 \quad 6.16 \mathrm{E}+00$

$1.28 \mathrm{E}+01 \quad 2.54 \mathrm{E}+00$

1.79E+01 1.49E+01

3.88E+00 1.30E+01

1.00E-02 1.01E-01

$1.00 \mathrm{E}-01 \quad 1.20 \mathrm{E}+00$

2.00E-03 2.68E-01

1. 38E+00

9. $32 \mathrm{E}+00 \quad 4.19 \mathrm{E}+00$

6. 15E-01

8. 00E-02

$1.00 \mathrm{E}-03$

$1.07 E+01 \quad 1.03 E+01$

2.00E-01 2.15E-01

8. 63E-01

1.00E-02 4.28E-01

4.60E-01 5.58E-01

2.04E+00

$3.59 E+01 \quad 8.48 E+01$

1. 32E+00

8.99E-01

$1.50 E-01 \quad 4.96 E+00$

1. $70 \mathrm{E}+00 \quad 1.60 \mathrm{E}+00$

2.90E+00 1.70E-01

1.93E-01

1. 10E-02

5.83E+00 2.75E+00

1. 86E-01

7. 20E-02

5. 19E-01

N/A=NOT APPLICABLE

$N / D=N O T$ DETECTED

N/R=NOT REPORTED 
INSTALLATION : PI LGRIM

EFFLUENT AND WASTE DISPOSAL ANNUAL REPORT FOR YEAR 1981 SOLID EFFLUENTS

ESTIMATE OF MAJOR NUCLIDE COMPOSITION(BY TYPE OF WASTE) SR-90 $\mathrm{ZN}-65$ ZR-NB-95

\begin{tabular}{|c|c|c|}
\hline & JAN-JUNE & JULY-DEC \\
\hline 8 & & 1. 20E-02 \\
\hline & $7.35 \mathrm{E}+00$ & $3.01 \mathrm{E}-01$ \\
\hline & $4.00 \mathrm{E}-02$ & \\
\hline
\end{tabular}

TYPE OF WASTE

A. SPENT RESINS, FILTER SLUDGES, EVAPORATOR BOTTOMS, ETC.

B. DRY COMPRESSIBLE WASTE, CONTAMINATED EQUI PMENT, ETC.

4. 00E-02

UNIT YEAR TOTAL

M3 $2.10 \mathrm{E}+02$

CI $\quad 8.59 \mathrm{E}+02$

M3 8.54E+02

CI $\quad 7.88 E+01$

C. IRRADIATED COMPONENTS, CONTROL RODS, ETC

M3

CI

D. OTHER

M3

CI

N/A=NOT APPLICABLE

$N / D=N O T$ DETECTED

N/R =NOT REPORTED 
EFFLUENT AND WASTE DISPOSAL ANNUAL REPORT FOR YEAR 1981 AIRBORNE AND LIQUID EFFLUENTS

UNIT NUMBER: 1

TYPE : PWR

DOCKET NO . : 50-266

COOLING WATER SOURCE: LAKE MICHIGAN UNIT NUMBER : 2

TYPE : PWR

DOCKET NO . : 50-301

COOLING WATER SOURCE: LAKE MICHIGAN

\begin{tabular}{|c|c|}
\hline AIRBORNE EFFLUENTS & \\
\hline NUCLIDES RELEASED & ACTIVITY (CI) \\
\hline$F-18$ & $1.68 E-07$ \\
\hline NA-24 & $3.45 \mathrm{E}-08$ \\
\hline AR-41 & $3.85 E+01$ \\
\hline CR-5 1 & $5.38 E-06$ \\
\hline$M N-54$ & $1.19 \mathrm{E}-06$ \\
\hline $\mathrm{CO}-58$ & $8.20 E-05$ \\
\hline $\mathrm{CO}-60$ & $1.96 \mathrm{E}-04$ \\
\hline KR-85 & $2.00 E+01$ \\
\hline$K R-85 M$ & 4. $15 \mathrm{E}+01$ \\
\hline KR-87 & $2.87 \mathrm{E}+01$ \\
\hline KR-88 & $6.88 \mathrm{E}+01$ \\
\hline RB-88 & $1.76 \mathrm{E}-01$ \\
\hline SR-89 & $9.50 \mathrm{E}-07$ \\
\hline SR-90 & $2.15 \mathrm{E}-07$ \\
\hline NB-95 & $1.18 \mathrm{E}-04$ \\
\hline ZR-95 & $9.77 \mathrm{E}-05$ \\
\hline ZR-97 & 1. $27 E-07$ \\
\hline RU -103 & $5.13 E-06$ \\
\hline CD- 109 & $6.72 E-09$ \\
\hline AG- $110 M$ & $1.24 \mathrm{E}-09$ \\
\hline SN-113 & 3.01E-06 \\
\hline SB-125 & $1.28 \mathrm{E}-08$ \\
\hline I-131 & $4.49 \mathrm{E}-03$ \\
\hline$X E-131 M$ & $1.04 \mathrm{E}-01$ \\
\hline I-132 & $4.40 \mathrm{E}-03$ \\
\hline TE-1 32 & $3.00 \mathrm{E}-06$ \\
\hline BA-133 & $9.34 \mathrm{E}-09$ \\
\hline I - 193 & $1.08 E-03$ \\
\hline$X E-133$ & 1.53E+02 \\
\hline$X E-133 M$ & $6.57 E+00$ \\
\hline CS-134 & $4.01 \mathrm{E}-05$ \\
\hline I-134 & $2.96 \mathrm{E}-04$ \\
\hline $1-135$ & $9.80 E-05$ \\
\hline$X E-135$ & 1. $78 E+02$ \\
\hline$X E-135 M$ & $2.57 E+01$ \\
\hline CS-137 & $6.90 \mathrm{E}-05$ \\
\hline CS-138 & $1.53 \mathrm{E}-02$ \\
\hline XE-138 & 4.94E+01 \\
\hline CE-139 & 1.03E-06 \\
\hline LA- 140 & $1.95 \mathrm{E}-09$ \\
\hline
\end{tabular}

LICENSEE : WISCONS IN ELECTRIC POWER COMPANY LICENSED POWER (MWT) : 1518.50 INITIAL CRITICALITY : $11 / 02 / 70$

LICENSEE : WISCONS IN ELECTRIC POWER COMPANY LICENSED POWER (MWT) : 1518.50 INITIAL CRITICALITY:05/30/72

N/A $=$ NOT APPLICABLE

$N / D=N O T$ DETECTED

N/R=NOT REPORTED 
EFFLUENT AND WASTE DISPOSAL ANNUAL REPORT FOR YEAR 1981 AIRBORNE AND LIQUID EFFLUENTS

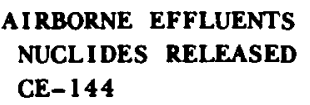

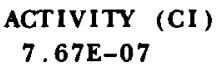

VOLUME OF LIQUID WASTE RELEASED (PRIOR TO DILUTION) LITERS 2.78E+08 VOLUME OF DILUTION WATER USED DURING PERIOD 
INSTALLATION : POINT BEACH

EFFLUENT AND WASTE DISPOSAL ANNUAL REPORT FOR YEAR 1981 SOLID EFFLUENTS

SOLID WASTE DISPOSITION

NUMBER OF SHIPMENTS MODE OF TRANSPORTATION

24

TRUCK

DESTINATION

N/R

IRRADIATED FUEL SHI PMENTS (DISPOS ITION)

NUMBER OF SHIPMENTS MODE OF TRANSPORTATION

DESTINATION

0

TYPE OF WASTE

A. SPENT RESINS, FILTER SLUDGES, EVAPORATOR

UNIT YEAR TOTAI BOTTOMS, ETC.

B. DRY COMPRESS IBLE WASTE, CONTAMINATED

MS 1.77E+02

CI $\quad 4.87 \mathrm{E}+02$

EQUI PMENT, ETC.

M3

CI

M3

C. IRRADIATED COMPONENTS, CONTROL

CI

RODS, ETC.

D. OTHER

M3

CI

N/A=NOT APPLICABLE

N/D=NOT DETECTED

N/R=NOT REPORTED 
EFFLUENT AND WASTE DISPOSAL ANNUAL REPORT FOR YEAR 1981 AIRBORNE AND LIQUID EFFLUENTS

UNIT NUMBER: 1

TYPE : PWR

DOCKET NO. : 50-282

COOLING WATER SOURCE:MISSISSIPPI RIVER UNIT NUMBER : 2

TYPE: $\quad$ PWR

DOCKET NO. : 50-306

COOLING WATER SOURCE:MISSISSIPPI RIVER

AIRBORNE EFFLUENTS

NUCLIDES RELEASED

- AR-41

CO-58

CO-60

$\mathbf{K R}-\mathbf{8 5}$

KR-85M

KR-87

$\mathrm{KR}-88$

RB-88

Y-88

AG-110M

I-131

$1-133$

$X E-133$

$X E-133 M$

$X E-135$

$\mathrm{XE}-135 \mathrm{M}$

CS-138

$\mathrm{XE}-138$

CE-144

LIQUID EFFLUENTS

NUCLIDES RELEASED

MN-54

CO-58

CO-60

ZR-NB-97

SB-124

XE-133

$\mathrm{XE}-133 \mathrm{M}$

XE- 135

CS-137

BA-LA-140

TRITIUM

TOTAL AIRBORNE RELEASE

TOTAL LIQUID RELEASE.
ACTIVITY (CI)

3. $17 \mathrm{E}+00$

4. 20E-05

1. 39E-05

1. $21 \mathrm{E}-01$

3. 49E-01

6. $06 E-01$

1. 88E+00

1. $48 \mathrm{E}+00$

3. 52E-02

3. 06E-05

3. 61 E-04

7. 37E-04

3. 39E+01

1. 69E-01

4. 19E+00

4. 70E-01

1. 70E+00

1. 70E +00

1. 82E-06

ACTIVITY (CI)

5. 31E-05

3. 84E-03

1. 38E-03

1. 13E-05

6. 76E-04

1. 35E-02

9. 70E-05

3. 38E-04

1. 78E-05

2.82E-05

(CI)

7. $35 \mathrm{E}+01$

5. 62E+02
LICENSEE: NORTHERN STATES POWER LICENSED POWER (MWT) : $\mathbf{1 6 5 0 . 0 0}$ INITIAL. CRITICALITY: $12 / 01 / 73$

LICENSEE : NORTHERN STATES POWER LICENSED POWER (MWT) : 1650.00 INITIAL CRITICALITY : $12 / 17 / 74$

N/A=NOT APPLICABLE

N/D $=$ NOT DETECTED

N/R=NOT REPORTED 
EFFLUENT AND WASTE DISPOSAL ANNUAL REPORT FOR YEAR 1981 AIRBORNE AND LIQUID EFFLUENTS

$\begin{array}{llll}\text { VOLUME OF LIQUID WASTE RELEASED (PRIOR TO DILUTION) } & \text { LITERS } & 1.65 E+08 \\ \text { VOLUME OF DILUTION WATER USED DURING PERIOD } & \text { LITERS } & 1.22 E+12\end{array}$


EFFLUENT AND WASTE DISPOSAL ANNUAL REPORT FOR YEAR 1981 SOLID EFFLUENTS

SOLID WASTE DISPOSITION NUMBER OF SHIPMENTS MODE OF TRANSPORTATION 18 TRUCK

IRRADI ATED FUEL SHI PMENTS (DIS POS ITION) NUMBER OF SHIPMENTS MODE OF TRANSPORTATION

ESTIMATE OF MAJOR NUCLIDE COMPOSITION(BY TYPE OF WASTE)

A

CO-58

CO-60

CS-134

CS-137

$M N-54$

CO-58

CO-60

CS-134

CS-137

MISC

MN-54

C

NONE

D

C0-58

Co-60
DESTINATION

RICHLAND, WA

DESTINATION

JAN-JUNE JULY-DEC
2. 40E+00
2.00E+00
$7.96 \mathrm{E}+01$
3. $60 \mathrm{E}+01$
3. $00 \mathrm{E}+00$
1. $50 \mathrm{E}+01$
1. $15 \mathrm{E}+01$
4. $50 \mathrm{E}+01$
3. $50 \mathrm{E}+00$
2. $00 \mathrm{E}+00$
7. $40 \mathrm{E}+00$
1. $50 \mathrm{E}+01$
$6.45 \mathrm{E}+01$
3. $50 \mathrm{E}+01$
2. $00 \mathrm{E}+00$
$1.00 E+01$
1. $95 \mathrm{E}+01$
6. $60 \mathrm{E}+00$
$2.50 E+01$
1. $50 \mathrm{E}+01$
N/A
8. $50 \mathrm{E}+00$
9. $15 E+01$

UNIT YEAR TOTAL

M3 2.07E+01

CI $4.36 \mathrm{E}+01$

M3 $1.82 E+02$

CI $1.27 E+01$

M3

CI

M3 $9.37 \mathrm{E}+01$

CI $\quad 9.60 \mathrm{E}-02$

N/A $=$ NOT APPLICABLE

N/D=NOT DETECTED

N/R=NOT REPORTED 
EFFLUENT AND WASTE DISPOSAL ANNUAL REPORT FOR YEAR 1981 AIRBORNE AND LIQUID EFFLUENTS

UNIT NUMBER : 1

TYPE: BWR

DOCKET NO . : 50-254

COOLING WATER SOURCE :MISS ISS I PPI RIVER

UNIT NUMBER : 2

TYPE: $\quad$ BWR

DOCKET NO. : 50-265

COOLING WATER SOURCE : MISS ISS I PPI RIVER

AIRBORNE EFFLUENTS

NUCLIDES RELEASED

CR-5 1

$\mathrm{MN}-54$

CO-58

$\mathrm{CO}-60$

$\mathrm{ZN}-65$

KR-85

KR-85M

KR-87

KR-88

SR-89

SR-90

RU- 103

AG- $110 \mathrm{M}$

SB-124

I - 131

1-193

$\mathrm{XE}-139$

CS-134

I -135

XE- 135

$\mathrm{XE}-135 \mathrm{M}$

CS-136

CS- 137

$\mathrm{XE}-138$

BA- 140

LA- 140

CE-141

CE-144

UNI DENT IFIED

LIQUID EFFLUENTS

NUCLIDES RELEASED

NA-24

CR-5 1

MN-54

CO-58

FE-59

$\mathrm{CO}-60$

$\mathrm{ZN}-65$

SR-89

N/A=NOT APPLICABLE

$N / D=N O T$ DETECTED

N/R=NOT REPORTED
LICENSEE : COMMONWEALTH EDISON AND IOWA-ILLINOIS GAS \& ELECTRIC LICENSED POWER (MWT) :2511.00

INITIAL CRITICALITY: $10 / 18 / 71$

LICENSEE : COMMONWEALTH EDISON AND IOWA-ILLINOIS GAS \& ELECTRIC LICENSED POWER (MWT) :2511.00

INITIAL CRITICALITY:04/26/72

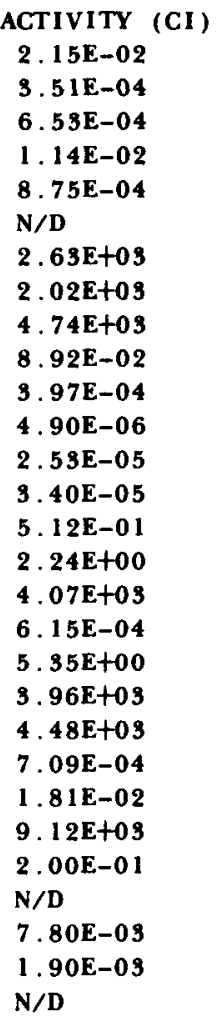

6. $21 \mathrm{E}-03$ 
EFFLUENT AND WASTE DISPOSAL ANNUAL REPORT FOR YEAR 1981

AIRBORNE AND LIQUID EFFLUENTS

$\begin{array}{ll}\text { LIQUID EFFLUENTS } & \\ \text { NUCLIDES RELEASED } & \text { ACTIVITY (CI) } \\ \text { SR-90 } & 1.28 E-03 \\ \text { SR-91 } & 1.85 E-03 \\ \text { SR-92 } & 9.93 E-05 \\ \text { NB-95 } & 2.80 E-05 \\ \text { ZR-95 } & \text { N/D } \\ \text { MO-99 } & 2.07 E-03 \\ \text { TC-99M } & 2.93 E-03 \\ \text { AG-110M } & \text { N/D } \\ \text { I-131 } & 1.22 E-02 \\ \text { I-133 } & 1.14 E-02 \\ \text { XE-133 } & 1.48 E+00 \\ \text { XE-133M } & 1.28 E-02 \\ \text { CS-134 } & 6.98 E-01 \\ \text { I-135 } & 5.62 E-03 \\ \text { XE-135 } & 2.48 E-01 \\ \text { CS-136 } & 1.25 E-04 \\ \text { CS-137 } & 2.29 E+00 \\ \text { CS-138 } & 2.60 E-03 \\ \text { BA-140 } & 5.43 E-03 \\ \text { LA-140 } & 1.44 E-03 \\ \text { CE-141 } & 2.67 E-04 \\ \text { NP-239 } & 1.10 E-04 \\ & \\ \text { TRITIUM } & \\ \text { TOTAL AIRBORNE RELEASE } & 8.59 E+01 \\ \text { TOTAL LIQUID RELEASE } & 1.19 E+01 \\ & \\ & \\ & \end{array}$

VOLUME OF LIQUID WASTE RELEASED (PRIOR TO DILUTION) LITERS 6.11E+06

VOLUME OF DILUTION WATER USED DURING PERIOD LITERS 3.08E+09

N/A=NOT APPLICABLE

$N / D=N O T$ DETECTED

N/R=NOT REPORTED 
EFFLUENT AND WASTE DISPOSAL ANNUAL REPORT FOR YEAR 1981 AIRBORNE AND LIQUID EFFLUENTS

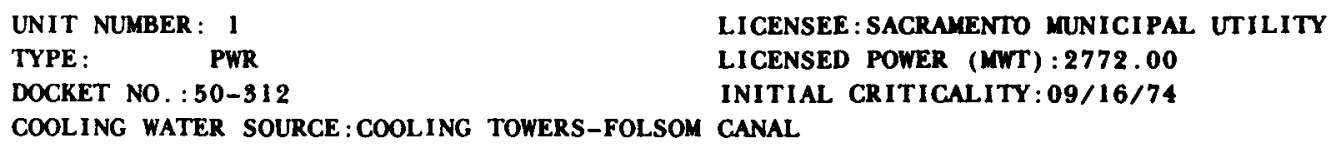

LIQUID EFFLUENTS NUCLIDES RELEASED

$N / A=$ NOT APPLICABLE

$N / D=N O T$ DETECTED

N/R=NOT REPORTED 
EFFLUENT AND WASTE DISPOSAL ANNUAL REPORT FOR YEAR 1981

AIRBORNE AND LIQUID EFFLUENTS

TRITIUM

TOTAL AIRBORNE RELEASE

TOTAL LIQUID RELEASE
(C1)

1. $41 \mathrm{E}+02$

8.35E+01
VOLUME OF LIQUID WASTE RELEASED (PRIOR TO DILUTION) VOLUME OF DILUTION WATER USED DURING PERIOD
LITERS 9.11E+07

LITERS $1.76 E+09$

N/A=NOT APPLICABLE

N/D=NOT DETECTED

N/R=NOT REPORTED 
INSTALLATION : RANCHO SECO

EFFLUENT AND WASTE DISPOSAL ANNUAL REPORT FOR YEAR 1981 SOLID EFFLUENTS

SOLID WASTE DISPOSITION

NUMBER OF SHIPMENTS MODE OF TRANSPORTATION

DESTINATION

2

TRUCK (SOLE USE)

BEATTY, NV

13

TRUCK (SOLE USE)

RICHLAND, WA

IRRADI ATED FUEL SHI PMENTS (DI SPOSITION)

NUMBER OF SHIPMENTS MODE OF TRANSPORTATION

DESTINATION

0

ESTIMATE OF MAJOR NUCLIDE COMPOSITION(BY TYPE OF WASTE)

A

NOT APPLICABLE

B

C0-58

C0-60

CR-5 1/AG- $110 \mathrm{M}$

MN-54

C

NOT APPLICABLE

D

AG-110M

CO-58

$\mathrm{CO}-60$

CR-51/NB-95

CS $-134 / C S-137$

MN-54

\begin{tabular}{|c|c|}
\hline JAN-JUNE & JULY-DEC \\
\hline$\%$ & $\mathrm{~N} / \mathrm{A}$ \\
\hline$\%$ & 3.00E+01 \\
\hline$\%$ & $5.30 E+01$ \\
\hline$\%$ & $5.00 \mathrm{E}+00$ \\
\hline$\%$ & 1. $20 \mathrm{E}+01$ \\
\hline$\%$ & $\mathbf{N} / \mathbf{A}$ \\
\hline$\%$ & $3.00 E+00$ \\
\hline$\%$ & $2.70 E+01$ \\
\hline$\varnothing$ & $5.80 E+01$ \\
\hline$\%$ & $2.00 E+00$ \\
\hline$\%$ & $1.00 \mathrm{E}+00$ \\
\hline$\%$ & 8.00E+00 \\
\hline
\end{tabular}

TYPE OF WASTE

A. SPENT RESINS, FILTER SLUDGES, EVAPORATOR

UNIT YEAR TOTAL BOTTOMS, ETC.

B. DRY COMPRESS IBLE WASTE, CONTAMINATED EQUI PMENT, ETC.

C. IRRADIATED COMPONENTS, CONTROL RODS, ETC.

D. OTHER

$\begin{array}{ll}\text { M3 } & 0.00 E+00 \\ \text { CI } & 0.00 E+00 \\ \text { M3 } & 1.22 E+02 \\ \text { CI } & 6.59 E+00 \\ \text { M3 } & 0.00 E+00 \\ \text { CI } & 0.00 E+00 \\ \text { M3 } & 1.09 E+02 \\ \text { CI } & 1.37 E+02\end{array}$


EFFLUENT AND WASTE DISPOSAL ANNUAL REPORT FOR YEAR 1981 AIRBORNE AND LIQUID EFFLUENTS

UNIT NUMBER : 2

TYPE : PWR

DOCKET NO. : 50-261

COOLING WATER SOURCE: ROBINSON INPOUNDMENT

\section{AIRBORNE EFFLUENTS}

NUCLIDES RELEASED

F-1 8

AR-41

CO-58

CO-60

KR-85M

KR-87

KR-88

NB-97

I -13 |

I-133

XE-133

$\mathrm{XE}-133 \mathrm{M}$

XE-135

XE-135M

CS -137

\section{LIQUID EFFLUENTS}

NUCLIDES RELEASED

F-1 8

NA-24

AR-41

CR-51

MN-54

$\mathrm{CO}-57$

CO-58

FE-59

$\mathrm{CO}-60$

$\mathrm{ZN}-65$

KR-85M

KR-87

SR-89

SR-90

SR-92

NB-95

ZR-95

NB-97

MO-99

TC-99M

AG-110M

SN-113

SB-124

I -131

I -133

$\mathrm{XE}-139$

N/A=NOT APPLICABLE

N/D=NOT DETECTED

N/R=NOT REPORTED

$$
\begin{aligned}
& \text { ACTIVITY }(C I) \\
& 1.10 \mathrm{E}-05 \\
& 4.78 \mathrm{E}+01 \\
& 3.75 \mathrm{E}-05 \\
& 2.74 \mathrm{E}-04 \\
& 2.60 \mathrm{E}+00 \\
& 1.87 \mathrm{E}+00 \\
& 1.35 \mathrm{E}+00 \\
& 1.28 \mathrm{E}-05 \\
& 2.00 \mathrm{E}-05 \\
& 7.86 \mathrm{E}-05 \\
& 3.49 \mathrm{E}+02 \\
& 2.89 \mathrm{E}+00 \\
& 1.04 \mathrm{E}+02 \\
& 4.37 \mathrm{E}+00 \\
& \text { 8.58E-07 }
\end{aligned}
$$

2.56E-03

1. $56 \mathrm{E}+00$

3. $96 \mathrm{E}-02$

1. 29E-03

3. $16 \mathrm{E}-03$

1. $28 \mathrm{E}-07$

3. $94 \mathrm{E}-02$

5. 03E-05

4. $06 \mathrm{E}-02$

4. $57 \mathrm{E}-04$

1. $25 \mathrm{E}-04$

2. $51 \mathrm{E}-06$

1. 06E-03

5. 60E-03

$2.14 \mathrm{E}-06$

$1.40 \mathrm{E}-03$

$6.76 \mathrm{E}-04$

8. 46E-04

8. 73E-04

8. 49E-04

3. $35 \mathrm{E}-04$

2. 36E-05

9.91E-05

3. 90E-03

1. $71 \mathrm{E}-01$

1. 20E-02
ACTIVITY (CI)
LICENSEE: CAROLINA POWER \&LIGHT

LICENSED POWER (MWT) : 2300.00

INITIAL CRITICALITY:09/20/70 
EFFLUENT AND WASTE DISPOSAL ANNUAL REPORT FOR YEAR 1981 AIRBORNE AND LIQUID EFFLUENTS

$\begin{array}{lc}\text { LIQUID EFFLUENTS } & \\ \text { NUCLIDES RELEASED } & \text { ACTIVITY (CI) } \\ \text { CS-134 } & 7.45 E-04 \\ \text { XE-135 } & 9.70 E-03 \\ \text { XE-195M } & 4.67 E-04 \\ \text { CS-137 } & 1.12 E-02 \\ \text { CE-144 } & 7.34 E-06 \\ & \\ \text { TRITIUM } & (\mathrm{CI}) \\ \text { TOTAL AIRBORNE RELEASE } & 1.06 E+01 \\ \text { TOTAL LIQUID RELEASE } & 1.86 E+02\end{array}$

VOLUME OF LIQUID WASTE RELEASED (PRIOR TO DILUTION) VOLUME OF DILUTION WATER USED DURING PERIOD $\begin{array}{ll}\text { LITERS } & 1.09 E+08 \\ \text { LITERS } & 8.42 E+11\end{array}$

N/A =NOT APPLICABLE

$N / D=$ NOT DETECTED

N/R $=$ NOT REPORTED 
INSTALLATION : H.B. ROBINSON

EFFLUENT AND WASTE DISPOSAL ANNUAL REPORT FOR YEAR 1981 SOLID EFFLUENTS

SOLID WASTE DISPOSITION

NUMBER OF SHIPMENTS MODE OF TRANSPORTATION

54

TRUCK

DESTINATION

BARNWELL, S.C.

IRRADIATED FUEL SHI PMENTS (DISPOSITION)

NUMBER OF SHIPMENTS MODE OF TRANSPORTATION

DESTINATION

0

2 RAIL

SOUTHPORT, N.C.

ESTIMATE OF MAJOR NUCLIDE COMPOSITION(BY TYPE OF WASTE)

A

AG-110M

CO-58

$\mathrm{CO}-60$

CR-51

CS-137

$\mathrm{MN}-54$

NB-95

NB-97

OTHERS

B

SB-124

CO-58

Co-60

CR-51

CS-137

MN-54

NB-97

OTHERS

RAIL

JAN-JUNE JULY-DEC

3. 18E-02

1. 08E-01

5. 88E-01

3.93E-0I

4. 99E-01

2. $50 \mathrm{E}-02$

3. 18E-02

$2.50 \mathrm{E}-02$

1. 43E-01

3. 24E-03

3. 18E-02

2.12E-02 3.18E-02

1. 76E-02

7. $95 \mathrm{E}-02$

1. 27E-01

1. 49E-01

1. 30E-01

2. $02 \mathrm{E}+00$

3.07E-01

9. 88E-03

1. 56E-02

3. 40E-02

1. 56E-02

3. 12E-02

2. 08E-02

TYPE OF WASTE
A. SPENT RESINS, FILTER SLUDGES, EVAPORATOR BOTTOMS , ETC.
B , DRY COMPRESS IBLE WASTE, CONTAMINATED EQUI PMENT, ETC.
C. IRRADIATED COMPONENTS, CONTROL RODS, ETC
D. OTHER

$\begin{array}{lc}\text { UNIT } & \text { YEAR TOTAL } \\ \text { M3 } & 2.50 E+02 \\ \text { CI } & 4.99 E+00 \\ \text { M3 } & 6.52 E+02 \\ \text { CI } & 1.38 E+01 \\ \text { M3 } & \\ \text { CI } & \\ \text { M3 } & \\ \text { CI } & \end{array}$

N/A=NOT APPLICABLE

$N / D=N O T$ DETECTED

$N / R=$ NOT REPORTED 
EFFLUENT AND WASTE DISPOSAL ANNUAL REPORT FOR YEAR 1981 AIRBORNE AND LIQUID EFFLUENTS

UNIT NUMBER: 1

TYPE: $\quad$ PWR

DOCKET NO. : 50-272

COOLING WATER SOURCE: DELAWARE RIVER
LICENSEE : PSEAG

LICENSED POWER (MWT) : 3398.00

INITIAL CRITICALITY: $12 / 11 / 76$
AIRBORNE EFFLUENTS

NUCLIDES RELEASED

CS-139

F-1 8

AR-4 1

MN-54

$\mathrm{CO}-58$

CO-60

KR-85

$\mathrm{KR}-85 \mathrm{M}$

KR-87

KR-88

RB-88

Y-88

I-131

I-133

XE-133

XE-1 133M

XE-135

XE-1 135M

CS-137

CS-138

UNIDENTI F IED

LIQUID EFFLUENTS

NUCLIDES RELEASED

H-3

NA-24

CR-5 1

MN -54

CO-58

FE-59

CO-60

NB-95

ZR-95

MO-99

SN-1 13

SB-1 24

SB-1 25

TE-125M

I-191

I-133

XE- 133

$\mathrm{XE}-133 \mathrm{M}$

CS-134

XE-135
ACTIVITY (CI)

6.99E-04

3. 09E-04

2.43E-03

4. 12 E-03

2.12E-07

3. 80E-01

4. 62E-01

2.02E+00

$6.36 \mathrm{E}-03$

2.76E-02

8. 31E-02

4. 44E-03

9.31E-03

$1.64 \mathrm{E}-03$

6. 24E+02

2. 91 E+00

5. 24E+01

2. 99E-01

4 . 63E-05

5. 82E-04

3. $81 \mathrm{E}+02$

ACTIVITY (CI)

4.93E+02

2. 40E-02

5. 92E-02

$7.39 \mathrm{E}-02$

4.51E-01

3. $75 E-03$

3. 20E-01

1. 81E-02

$1.04 \mathrm{E}-02$

2. 52E-04

8.91E-04

6. 15E-04

2. 95E-01

5. 62E-03

1. 10E-01

8. 80E-02

5. 38E+00

5. 24E-02

5. 95E-02

9.64E-02

$N / A=N O T$ APPLICABLE

$N / D=N O T$ DETECTED

N/R=NOT REPORTED 
EFFLUENT AND WASTE DISPOSAL ANNUAL REPORT FOR YEAR 1981 AIRBORNE AND LIQUID EFFLUENTS

$\begin{array}{lc}\text { LIQUID EFFLUENTS } & \\ \text { NUCLIDES RELEASED } & \text { ACTIVITY (CI) } \\ \text { CS-136 } & 1.42 E-03 \\ \text { CS-137 } & 7.60 E-02 \\ \text { CE-144 } & 5.98 E-04 \\ & \\ \text { TRITIUM } & \text { (CI) } \\ \text { TOTAL AIRBORNE RELEASE } & 2.74 E+00 \\ \text { TOTAL LIQUID RELEASE } & 4.93 E+02\end{array}$

VOLUME OF LIQUID WASTE RELEASED (PRIOR TO DILUTION) LITERS 1.29E+07 VOLUME OF DILUTION WATER USED DURING PERIOD LITERS $3.35 \mathrm{E}+10$ 
EFFLUENT AND WASTE DISPOSAL ANNUAL REPORT FOR YEAR 1981 AIRBORNE AND LIQUID EFFLUENTS

UNIT NUMBER: 2

TYPE : PWR

DOCKET NO , :50-311

COOLING WATER SOURCE : DELAWARE RIVER

AIRBORNE EFFLUENTS

NUCLIDES RELEASED

F-18

MN-54

CO-58

CO-60

KR-85

KR-85M

SR-85

KR-88

I-131

I-133

$X E-133$

$\mathrm{XE}-133 \mathrm{M}$

CS-134

$\mathrm{XE}-135$

$\mathrm{XE}-135 \mathrm{M}$

CS-137

ACTIVITY (CI)

2. $96 \mathrm{E}-06$

2.39E-04

2. 12E-07

4. $60 \mathrm{E}-0 \mathrm{3}$

$1.65 \mathrm{E}-01$

1. $99 \mathrm{E}+00$

$6.33 E-04$

1. $69 \mathrm{E}-03$

8. 36E-04

$1.82 \mathrm{E}-05$

5. $42 \mathrm{E}+02$

3. 15E-01

1.05E-06

1. 38E+01

2. 99E-01

8.01E-06

LIQUID EFFLUENTS NUCLIDES RELEASED H-3

NA-24

CR-51

MN-54

CO-58

FE-59

Co-60

NB-95

ZR-95

MO-99

SN-113

SB-1 24

SB- 125

TE-125M

1-131

I-193

$\mathrm{XE}-133$

$\mathrm{XE}-139 \mathrm{M}$

CS-134

$\mathrm{XE}-135$

CS-137

CE-139

W-187

ACTIVITY (CI)
8. 43E+02
2.05E-01
$9.46 \mathrm{E}-02$
4. 43E-02
8. $12 \mathrm{E}-01$
5.84E-03
2.56E-01
1. 50E-02
1. 05E-02
7. $41 \mathrm{E}-0 \mathrm{~S}$
2.65E-04
9.07E-04
8.37E-03
3. $77 \mathrm{E}-02$
2. $57 \mathrm{E}-02$
6. $09 \mathrm{E}-04$
5. 59E+00
$5.65 \mathrm{E}-02$
1. $79 \mathrm{E}-02$
6. $99 \mathrm{E}-02$
2.94E-02
1. 45E-04
$2.49 \mathrm{E}-04$

\author{
LICENSEE : PSE\&G \\ LICENSED POWER (MWT) : 3338.00 \\ INITIAL CRITICALITY: 08/02/80
}

N/A=NOT APPLICABLE

$N / D=$ NOT DETECTED

$N / R=$ NOT REPORTED 
EFFLUENT AND WASTE DISPOSAL ANNUAL REPORT FOR YEAR 1981 AIRBORNE AND LIQUID EFFLUENTS
TRITIUM
(CI)
TOTAL AIRBORNE RELEASE 1.13E+00
TOTAL LIQUID RELEASE
8. 43E+02

VOLUME OF LIQUID WASTE RELEASED (PRIOR TO DILUTION)

LITERS $1.70 \mathrm{E}+07$

VOLUME OF DILUTION WATER USED DURING PERIOD

LITERS

5. $20 E+10$

N/A $=$ NOT APPLICABLE

$N / D=N O T$ DETECTED

N/R $=$ NOT REPORTED 
INSTALLATION: SALEM $1 \& 2$

EFFLUENT AND WASTE DISPOSAL ANNUAL REPORT FOR YEAR 1981 SOLID EFFLUENTS

SOLID WASTE DISPOSITION

NUMBER OF SHIPMENTS MODE OF TRANSPORTATION

86

4

TRUCK

TRUCK

NUMBER OF SHIPMENTS MODE OF TRANSPORTATION

$\mathbf{0}$

ESTIMATE OF MAJOR NUCLIDE COMPOSITION(BY TYPE OF WASTE)

A

$$
\begin{aligned}
& \text { CO-58 } \\
& \text { CO-60 } \\
& \text { FE-55 } \\
& M N-54 \\
& \\
& C O-58 \\
& C O-60 \\
& \text { FE-55 } \\
& M N-54
\end{aligned}
$$

B
DESTINATION

BARNWELL, S.C.

BEATTY, NV

DESTINATION

TYPE OF WASTE

A. SPENT RESINS, FILTER SLUDGES, EVAPORATOR BOTTOMS, ETC.

B . DRY COMPRESS I BLE WASTE, CONTAMINATED EQU I PMENT, ETC.

JAN-JUNE JULY-DEC

C. IRRADIATED COMPONENTS, CONTROL RODS, ETC.

D. OTHER

$\begin{array}{lll}* & 7.30 E+01 & 7.30 E+01 \\ \% & 1.50 E+01 & 1.50 E+01 \\ \% & 9.00 E+00 & 9.00 E+00 \\ \% & 3.00 E+00 & 3.00 E+00 \\ & & \\ \% & 7.30 E+01 & 7.30 E+01 \\ \% & 1.50 E+01 & 1.50 E+01 \\ \% & 9.00 E+00 & 9.00 E+00 \\ \% & 3.00 E+00 & 3.00 E+00\end{array}$

UNIT YEAR TOTAL

M3 2.34E+02

CI $1.10 \mathrm{E}+09$

M3 7.02E+02

CI 3.70E+01

M3

CI

M3

CI 
EFFLUENT AND WASTE DISPOSAL. ANNUAL. REPORT FOR YEAR 1981 AIRBORNE AND LIQUID EFFLUENTS

UNIT NUMBER: 1

TYPE : PWR

DOCKET NO . : 50-206

COOLING WATER SOURCE: PACIFIC OCEAN

AIRBORNE EFFLUENTS

NUCLIDES RELEASED

AR-4 1

CR-5 1

MN-54

CO-57

C0-58

$\mathrm{CO}-60$

KR-85

KR-85M

KR-87

KR-88

SR-89

SR-90

I-131

$\mathrm{XE}-131$

$X E-131 M$

$X E-133$

XE-133M

CS- 134

$\mathrm{XE}-135$

$X E-135 M$

CS-137

BA-LA- 140

LIQUID EFFLUENTS

NUCLIDES RELEASED

H-3

CR-5 I

$M N-54$

CO-57

CO-58

FE-59

C0-60

$\mathrm{ZN}-65$

KR-85

KR-85M

SR-89

SR-90

ZR-NB-95

MO-99

TC-99M

AG-110M

SB- 122

I-131

1-133

N/A =NOT APPLICABLE

$N / D=N O T$ DETECTED

N/R $=$ NOT REPORTED
LICENSEE : SOUTHERN CALIFORNIA EDISON AND SAN DIEGO GAS \& ELECTRI

LICENSED POWER (MWT) : 1347.00

INITIAL CRITICALITY:06/14/67

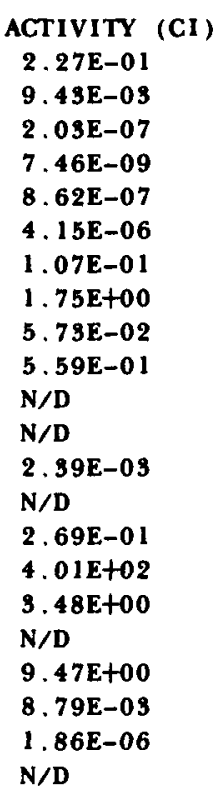

ACTIVITY (CI)
$2.97 \mathrm{E}+02$
$1.74 \mathrm{E}-01$
$1.99 \mathrm{E}-01$
$4.42 \mathrm{E}-03$
$2.67 \mathrm{E}-01$
$2.46 \mathrm{E}-02$
$2.14 \mathrm{E}+00$
$1.03 \mathrm{E}-02$
$2.67 \mathrm{E}-01$
N/D
N/D
N/D
$5.35 \mathrm{E}-03$
N/D
$1.79 \mathrm{E}-03$
$9.70 \mathrm{E}-03$
$1.72 \mathrm{E}-03$
$1.33 \mathrm{E}-02$
N/D


EFFLUENT AND WASTE DISPOSAL ANNUAL REPORT FOR YEAR 1981 AIRBORNE AND LIQUID EFFLUENTS

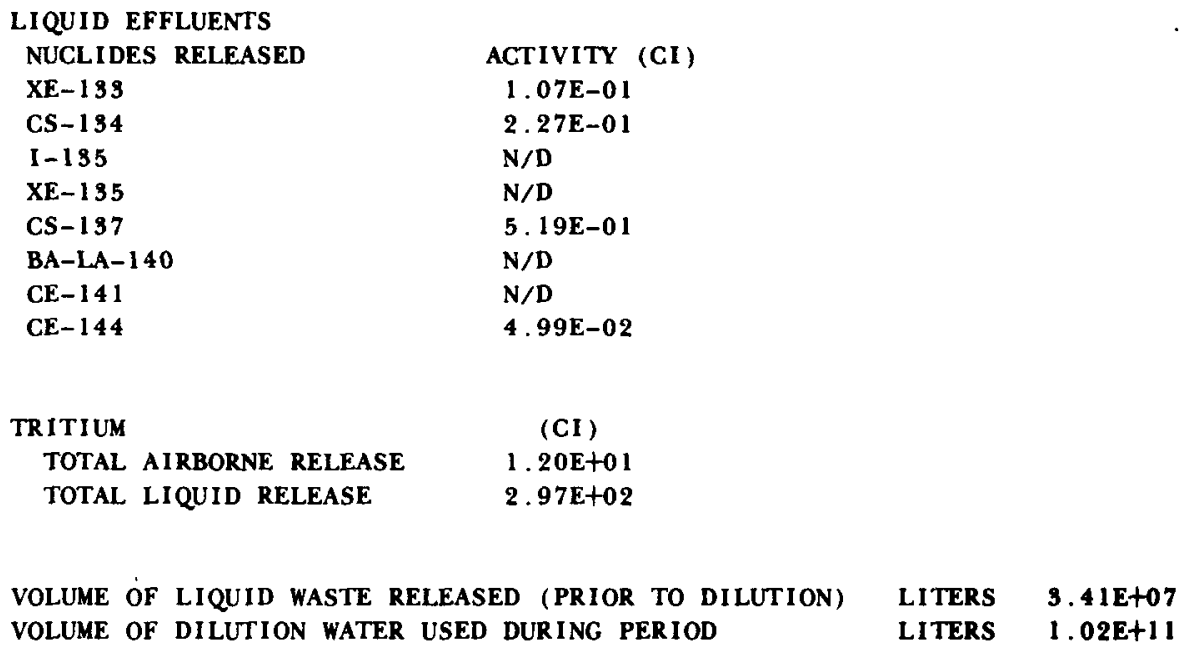

VOLUME OF LIQUID WASTE RELEASED (PRIOR TO DILUTION) LITERS 3.41E+07 
INSTALLATION : SAN ONOFRE

EFFLUENT AND WASTE DISPOSAL ANNUAL REPORT FOR YEAR 1981 SOLID EFFLUENTS

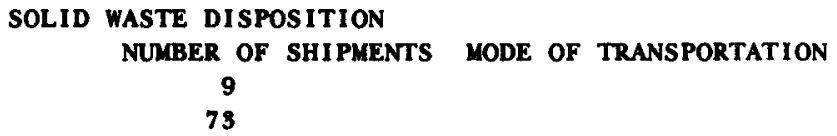

TYPE OF WASTE

A. SPENT RESINS, FILTER SLUDGES, EVAPORATOR BOTTOMS, ETC

B. DRY COMPRESS IBLE WASTE, CONTAMINATED EQUI PMENT, ETC

C. IRRADIATED COMPONENTS, CONTROL RODS, ETC.

D. OTHER
DESTINATION

RICHLAND WA

UNIT YEAR TOTAL

M3 $\quad 1.62 \mathrm{E}+03$

CI $1.26 E+03$

M3

CI

M3

CI

M3

CI 
EFFLUENT AND WASTE DISPOSAL ANNUAL REPORT FOR YEAR 1981 AIRBORNE AND LIQUID EFFLUENTS

\begin{abstract}
UNIT NUMBER: 1
TYPE: PWR
\end{abstract}

DOCKET NO . : 50-327

COOLING WATER SOURCE:TENNESSEE RIVER
LICENSEE : TENNESSEE VALLEY AUTHORITY

LICENSED POWER (MWT) : 2815.00

INITIAL. CRITICALITY: 07/05/80

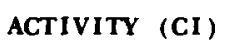

1. 22E-05

$1.75 \mathrm{E}-05$

1. $10 \mathrm{E}+01$

7. $41 \mathrm{E}-01$

5. 39E-04

$2.06 \mathrm{E}-07$

1. 24E-06

$1.96 E-13$

1. 16E-02

7.99E-04

2.31E+01

1. $54 \mathrm{E}-03$

8.87E+03

1. 59E+01

9.74E+01

ACTIVITY (CI)

1.22E-02

2. 63E-01

6. 57E-05

$6.06 \mathrm{E}-01$

3. $52 \mathrm{E}-01$

8. 60E-02

1. $01 \mathrm{E}+00$

3. 86E-02

6. 46E-02

2. 68E-04

8. 40E-04

1. $62 \mathrm{E}-03$

4. 88E-03

7. 89E-02

1. $57 \mathrm{E}-04$

1. $47 \mathrm{E}-04$

1. $63 \mathrm{E}-03$

1. $11 \mathrm{E}-03$

$6.60 \mathrm{E}-02$

6. 26E-02

8. 15E-07

2. 88E-03

2. 44E+00

2.81E-02

1.02E-02

N/A=NOT APPLICABLE

N/D=NOT DETECTED

N/R=NOT REPORTED 
EFFLUENT AND WASTE DISPOSAL ANNUAL REPORT FOR YEAR 1981 AIRBORNE AND LIQUID EFFLUENTS

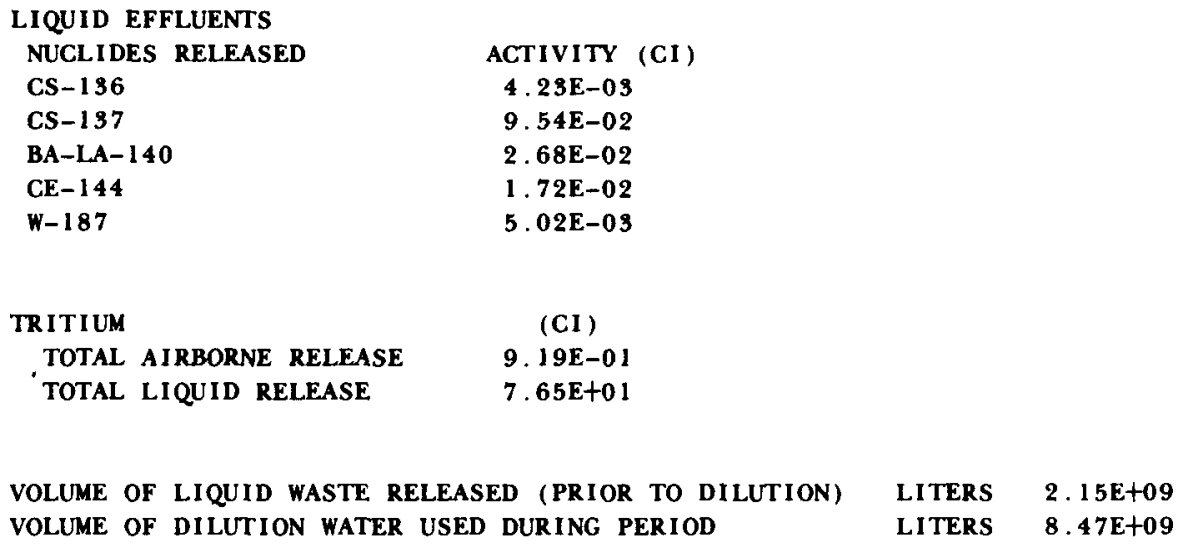


INSTALLATION : SEQUOYAH

EFFLUENT AND WASTE DISPOSAL ANNUAL REPORT FOR YEAR 1981 SOLID EFFLUENTS

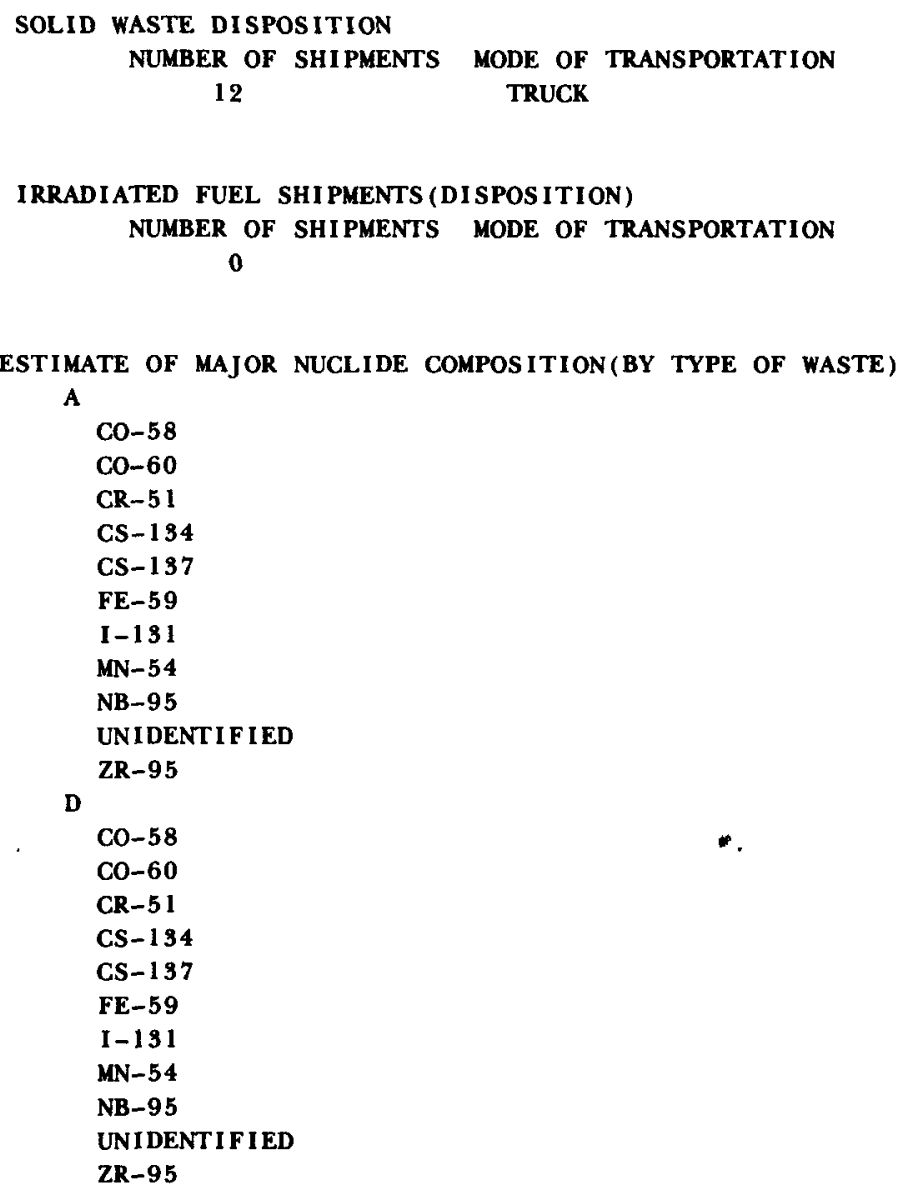

DESTINATION

BARNWELL SC

DESTINATION
JAN-JUNE JULY-DEC

\begin{tabular}{|c|c|c|}
\hline 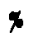 & 3. 15E+01 & $5.52 \mathrm{E}+01$ \\
\hline$\%$ & $3.24 E+00$ & $1.75 E+01$ \\
\hline * & $1.41 \mathrm{E}+01$ & $7.55 \mathrm{E}+00$ \\
\hline$\%$ & $1.51 \mathrm{E}-01$ & $9.59 \mathrm{E}-01$ \\
\hline * & $3.68 \mathrm{E}-01$ & 3. $00 E+00$ \\
\hline$\%$ & $9.57 \mathrm{E}-01$ & $6.24 \mathrm{E}-01$ \\
\hline \% & $4.22 \mathrm{E}+00$ & $4.61 \mathrm{E}+00$ \\
\hline$\%$ & $2.96 \mathrm{E}+01$ & $3.65 \mathrm{E}+00$ \\
\hline$\%$ & $1.34 \mathrm{E}+00$ & $1.98 E+00$ \\
\hline \% & $1.27 \mathrm{E}+01$ & 3. $79 E+00$ \\
\hline$\%$ & $9.40 \mathrm{E}-01$ & $1.08 \mathrm{E}-01$ \\
\hline$\%$ & $6.72 \mathrm{E}+01$ & $4.32 \mathrm{E}+01$ \\
\hline$\%$ & $7.38 E+00$ & $1.39 E+01$ \\
\hline$\%$ & $1.07 E+01$ & $7.17 \mathrm{E}+00$ \\
\hline$\%$ & & 5. $60 \mathrm{E}-01$ \\
\hline$\%$ & $5.96 \mathrm{E}-01$ & $2.21 \mathrm{E}-01$ \\
\hline$\%$ & $9.02 \mathrm{E}-01$ & $1.07 \mathrm{E}+00$ \\
\hline$\%$ & $6.99 \mathrm{E}-01$ & 2. $60 \mathrm{E}+0 \mathrm{I}$ \\
\hline 7 & $1.17 \mathrm{E}+01$ & $2.15 \mathrm{E}+00$ \\
\hline & & 1. $37 \mathrm{E}+00$ \\
\hline & $7.98 \mathrm{E}-01$ & $3.54 E+00$ \\
\hline & & $7.63 \mathrm{E}-01$ \\
\hline
\end{tabular}

TYPE OF WASTE

A. SPENT RESINS, FILTER SLUDGES, EVAPORATOR

UNIT YEAR TOTAL BOTTOMS, ETC.

B. DRY COMPRESS IBLE WASTE, CONTAMINATED EQUI PMENT, ETC.

C. IRRADIATED COMPONENTS, CONTROL RODS , ETC

D. OTHER BOXES AND DRUMS

$\begin{array}{ll}\text { M3 } & 3.81 E+01 \\ \text { CI } & 2.04 E+01 \\ \text { M3 } & \\ \text { CI } & \\ \text { M3 } & \\ \text { CI } & \\ \text { M3 } & 1.23 E+02 \\ \text { CI } & 8.80 E+00\end{array}$

N/A $=$ NOT APPLICABLE

$N / D=N O T$ DETECTED

N/R $=$ NOT REPORTED 
EFFLUENT AND WASTE DISPOSAL ANNUAL REPORT FOR YEAR 1981 AIRBORNE AND LIQUID EFFLUENTS

UNIT NUMBER: 1

TYPE : PWR

DOCKET NO . : 50-335

COOLING WATER SOURCE : ATLANTIC OCEAN

\section{AIRBORNE EFFLUENTS}

NUCLIDES RELEASED

AR-4 1

CO-58

CO-60

$\mathrm{KR}-85$

KR-85M

KR-87

$\mathbf{K R}-\mathbf{8 8}$

RB-88

ZR-95

I-131

$\mathrm{XE}-131 \mathrm{M}$

I-132

I-133

XE-1 133

$\mathrm{XE}-133 \mathrm{M}$

I -135

XE-1 35

XE-1 95M

$\mathrm{XE}-138$

BA-140

LA-140

\section{LIQUID EFFLUENTS}

NUCLIDES RELEASED

NA-24

AR-4 1

CR-5 1

MN-54

MN-56

CO-57

C0-58

FE-59

CO-60

NI -65

ZN-65

BR-82

KR-85

KR-85M

KR-87

KR-88

SR-89

SR-90

NB-95

ZR-95

N/A=NOT APPLICABLE $N / D=N O T$ DETECTED N/R=NOT REPORTED
LICENSEE : FLORIDA POWER \&LIGHT LICENSED POWER (MWT) $: 2560.00$ INITIAL CRITICALITY : 04/22/76

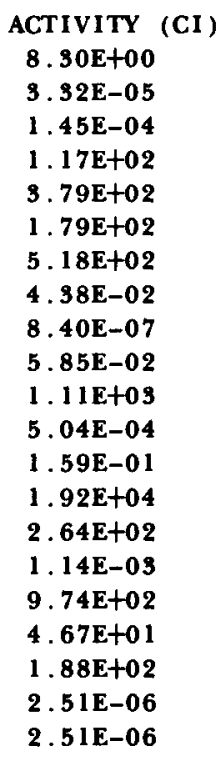

1. 45E-04

1. $17 \mathrm{E}+02$

3. $79 \mathrm{E}+02$

1. $79 E+02$

5. 18E+02

4. 38E-02

8. 40E-07

5. 85E-02

1. $11 \mathrm{E}+03$

$5.04 E-04$

1. 59E-01

$1.92 E+04$

2. $64 \mathrm{E}+02$

1. 14E-03

9. 74E+02

$4.67 \mathrm{E}+01$

1. 88E+02

2. $51 \mathrm{E}-06$

2.51E-06

ACTIVITY (CI)
4. 32E-04
6. 85E-04
2. 58E-01
2. 99E-02
8. 45E-03
$1,47 \mathrm{E}-03$
4. 69E-01
9. 64E-03
1. 19E+00
2. 88E-03
2.57E-03
9. 39E-05
8. $81 \mathrm{E}-02$
9. 68E-05
2.53E-05
6. 19E-05
2.53E-03
3. 68E-04
1. 03E-02
4. 58E-02 
EFFLUENT AND WASTE DISPOSAL ANNUAL REPORT FOR YEAR 1981 AIRBORNE AND LIQUID EFFLUENTS

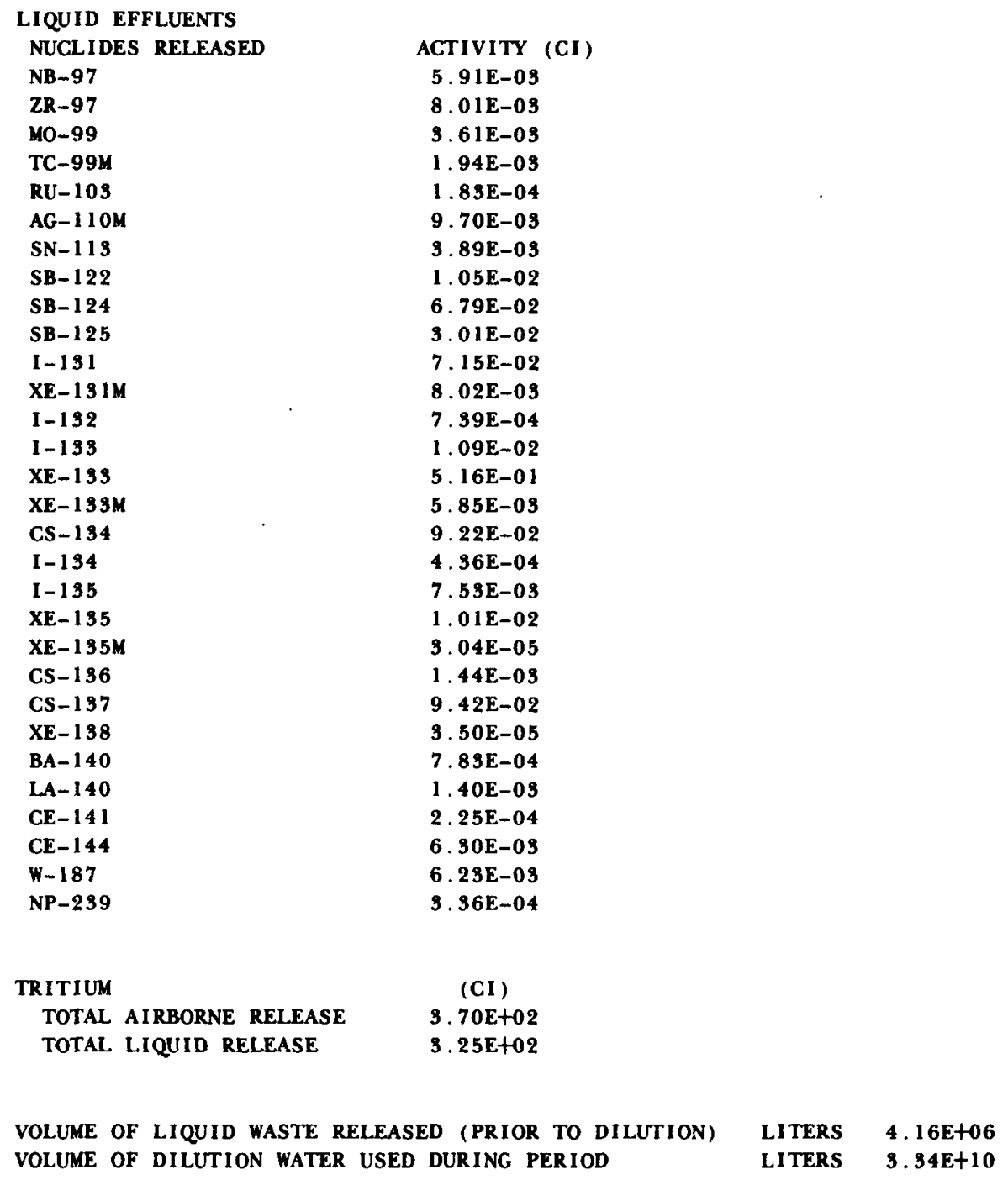


INSTALLATION:ST , LUCIE

EFFLUENT AND WASTE DISPOSAL ANNUAL REPORT FOR YEAR 1981 SOLID EFFLUENTS

SOLID WASTE DISPOSITION NUMBER OF SHIPMEŃNS MODE OF TRANSPORTATION 15 TRUCK

DESTINATION

BARNWELL SC

IRRADI ATED FUEL SHI PMENTS (DISPOSITION) NUMBER OF SHIPMENTS MODE OF TRANSPORTATION

DESTINATION

ESTIMATE OF MAJOR NUCLIDE COMPOSITION(BY TYPE OF WASTE)

A
C0-58
C0-60
CS-134
CS-137
MN- 54
CO-58
CO-60
CS-134
CS- 137
MN-54

JAN-JUNE JULY-DEC

$\begin{array}{ll}7.00 E+00 & 2.40 E+01 \\ 6.70 E+01 & 6.00 E+01 \\ 8.00 E+00 & 6.00 E+00 \\ 1.20 E+01 & 1.00 E+01 \\ 6.00 E+00 & \\ 1.00 E+01 & 1.50 E+01 \\ 6.20 E+01 & 3.50 E+01 \\ 7.00 E+00 & 1.50 E+01 \\ 1.80 E+01 & 3.00 E+01 \\ 3.00 E+00 & 5.00 E+00\end{array}$

TYPE OF WASTE

A. SPENT RESINS, FILTER SLUUDES, EVAPORATOR BOTTOMS, ETC.

B. DRY COMPRESS IBLE WASTE, CONTAMINATED EQUI PMENT, ETC.

C. IRRADIATED COMPONENTS, CONTROL RODS , ETC.

D. OTHER

UNIT YEAR TOTAL

M3 $1.89 \mathrm{E}+01$

CI $\quad 2.90 \mathrm{E}+02$

MS 2.31E+02

CI $6.12 \mathrm{E}+00$

MS

CI

M9

CI

N/A $=$ NOT APPLICABLE

$N / D=N O T$ DETECTED

N/R=NOT REPORTED 
EFFLUENT AND WASTE DISPOSAL ANNUAL REPORT FOR YEAR 1981 AIRBORNE AND LIQUID EFFLUENTS

UNIT NUMBER : 1

TYPE : $\quad$ PWR

DOCKET NO. : 50-280

COOLING WATER SOURCE : JAMES RIVER

UNIT NUMBER : 2

TYPE: $\quad$ PWR

DOCKET NO. : 50-281

COOLING WATER SOURCE: JAMES RIVER

AIRBORNE EFFLUENTS

NUCLIDES RELEASED

AR-41

CR-5 1

MN-54

CO-58

FE-59

CO-60

$\mathrm{ZN}-65$

KR-85

KR-85M

KR-88

RB-88

NB-95

ZR-95

RU-103

I-131

I-132

I-133

$\mathrm{XE}-133$

XE-133M

CS-134

I-135

XE- 135

CS-137

CS-138

\section{LIQUID EFFLUENTS}

NUCLIDES RELEASED

C-14

NA-24

AR-4 1

CR-5 1

MN-54

CO-57

CO-58

FE-59

CO-60

RB-88

SR-89

SR-90

NB-95
ACTIVITY (CI)

6. 85E-01

7. $11 \mathrm{E}-05$

8. 14E-05

7. 80E-04

2.37E-06

1. 90E-03

2.01E-07

6. $49 E+00$

$4.89 E+00$

5. 95E+00

2.77E-01

1. $61 \mathrm{E}-05$

6. 64E-06

1. 03E-06

4. 50E-02

1. 68E-07

1. 65E-02

1. 34E+04

6. 64E+01

1.23E-04

1. 50E-03

5. 67E+02

5. 48E-04

9. 33E-04

ACTIVITY (CI)

4. 69E-03

2. 10E+00

1. 29E-02

5. 25E-02

2. 21 E-02

1. 23E-03

1. 30E+00

7. 69E-04

1. 23E+00

2. 32E-01

2. 47 E-04

1.87E-05

5. 22E-03
LICENSEE: VIRGINIA ELECTRIC \&POWER

LICENSED POWER (MWT) :2441.00

INITIAL CRITICALITY : 07/01/72

LICENSEE : VIRGINIA ELECTRIC \&POWER

LICENSED POWER (MWT) :2441.00

INITIAL CRITICALITY : 03/07/73

N/A=NOT APPLICABLE

N/D=NOT DETECTED

N/R=NOT REPORTED 
EFFLUENT AND WASTE DISPOSAL ANNUAL REPORT FOR YEAR 1981 AIRBORNE AND LIQUID EFFLUENTS

LIQUID EFFLUENTS

NUCLIDES RELEASED

SB-1 24

SB- 125

I-131

XE-131M

$1-132$

$1-133$

$\mathrm{XE}-139$

$\mathrm{XE}-133 \mathrm{M}$

CS-134

$1-134$

$1-135$

XE-135

CS-196

CS-137

BA-LA- 140

CE-141

CE-144

TRITIUM

TOTAL AIRBORNE RELEASE

TOTAL LIQUID RELEASE

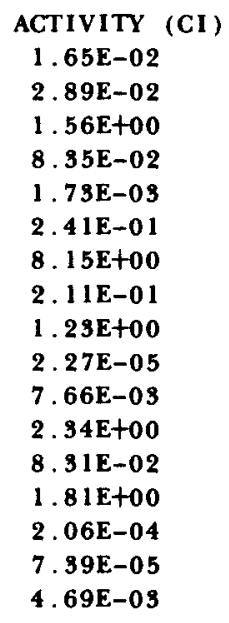

2. 89E-02

1. $56 \mathrm{E}+00$

8. 35E-02

1. 73E-03

2. $41 \mathrm{E}-01$

8. $15 E+00$

2.11E-01

1. $23 E+00$

2. $27 \mathrm{E}-05$

7. 66E-03

2. $94 \mathrm{E}+00$

8. 31E-02

1. $81 \mathrm{E}+00$

$2.06 \mathrm{E}-04$

7. 39E-05

4. 69E-03

(CI)

6. 19E+01

5. $31 E+02$

VOLUME OF LIQUID WASTE RELEASED (PRIOR TO DILUTION) VOLUME OF DILUTION WATER USED DURING PERIOD

LITERS 6.09E+08

LITERS 2.11E+12

N/A=NOT APPLICABLE

N/D =NOT DETECTED

N/R=NOT REPORTED 
INSTALLATION : SURRY

EFFLUENT AND WASTE DISPOSAL ANNUAL REPORT FOR YEAR 1981 SOLID EFFLUENTS

SOLID WASTE DISPOSITION

NUMBER OF SHI PMENTS MODE OF TRANSPORTATION

DESTINATION

126

BARNWELL SC

12

RICHLAND WA

TYPE OF WASTE

A. SPENT RESINS, FILTER SLUDGES, EVAPORATOR

UNIT YEAR TOTAL BOTTOMS, ETC.

B. DRY COMPRESS IBLE WASTE, CONTAMINATED

M3 2.80E+03

EQUI PMENT, ETC.

C. IRRADIATED COMPONENTS, CONTROL RODS, ETC.

CI $1.36 E+03$

CI

D. OTHER 
EFFLUENT AND WASTE DISPOSAL ANNUAL REPORT FOR YEAR 1981 AIRBORNE AND LIQUID EFFLUENTS

UNIT NUMBER: 1

TYPE : PWR

DOCKET NO. : 50-289

COOLING WATER SOURCE: SUSQUEHANNA RIVER

\section{AIRBORNE EFFLUENTS NUCLIDES RELEASED CO-60 \\ $\mathrm{KR}-85$ \\ SR-89 \\ SR-90 \\ CS-137}

\section{LIQUID EFFLUENTS}

NUCLIDES RELEASED

$$
\text { P-32 }
$$

MN-54

FE-55

CO-57

CO-58

C0-60

$\mathrm{ZN}-65$

SR-90

AG- $110 \mathrm{M}$

SB-125

CS-134

CS-137

TRITIUM

TOTAL AIRBORNE RELEASE

TOTAL LIQUID RELEASE
LICENSEE : METROPOLITAN EDISON \& JERSEY CENTRAL POWER LICENSED POWER (MWT) :2535.00 INITIAL CRITICALITY:06/05/74

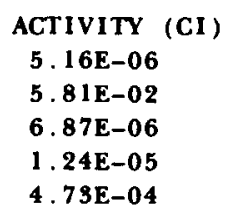

1. 23E-04

2. 22E-03

2.99E-06

3. $40 \mathrm{E}-05$

5.91E-03

4. 45E-06

7. $10 \mathrm{E}-05$

4. 36E-04

2. 10E-03

1. 29E-02

6. 32E-02

(CI )

1. 49E-02

7. $11 \mathrm{E}+00$

VOLUME OF LIQUID WASTE RELEASED (PRIOR TO DILUTION) LITERS 1.89E+09 VOLUME OF DILUTION WATER USED DURING PERIOD

LITERS 5. 58E +10 
INSTALLATION: THREE MILE ISLAND 1

EFFLUENT AND WASTE DISPOSAL ANNUAL REPORT FOR YEAR 1981 SOLID EFFLUENTS

SOLID WASTE DISPOSITION NUMBER OF SHI PMENTS

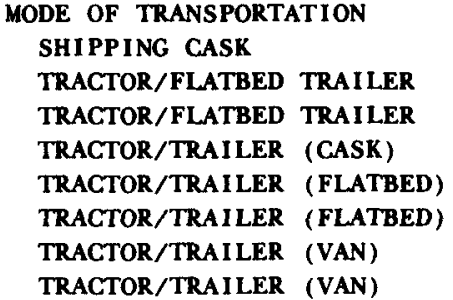

ESTIMATE OF MAJOR NUCLIDE COMPOSITYON(BY TYPE OF WASTE)

BA-137M

CO-60

CS-184

CS -137

B

AG-110M

BA-197M

CO-60

CS-134

CS-137

MN-54

\author{
DESTINATION \\ BARNWELL SC \\ BARNWELL SC \\ WASHINGTON STATE \\ BARNWELL SC \\ BARNWELL SC \\ RICHLAND WA \\ BARNWELL SC \\ WASHINGTON STATE
}

JAN-JUNE JULY-DEC
1. $80 \mathrm{E}+01$
3. $00 \mathrm{E}+01$
6. 00E-01
$1.35 \mathrm{E}+01$
1. $50 \mathrm{E}+01$
8. $50 \mathrm{E}+00$
5. $80 \mathrm{E}+01$
4. 12E+01
3. 10E+01
1. $80 \mathrm{E}+00$
7. $70 E+00$
3. 46E+01
1. $90 E+01$
8. 40E+00
3. $10 \mathrm{E}+0 \mathrm{I}$
$1.82 \mathrm{E}+01$
6. 10E +00
3. $63 \mathrm{E}+01$

UNIT YEAR TOTAL

TYPE OF WASTE

A. SPENT RESINS, FILTER SLUDGES, EVAPORATOR BOTTOMS, ETC.

B. DRY COMPRESSIBLE WASTE, CONTAMINATED EQUI PMENT, ETC.

C. IRRADI ATED COMPONENTS, CONTROL RODS, ETC.

D. OTHER

N/A=NOT APPLICABLE

$N / D=N O T$ DETECTED

$N / R=$ NOT REPORTED 
EFFLUENT AND WASTE DISPOSAL ANNUAL REPORT FOR YEAR 1981 AIRBORNE AND LIQUID EFFLUENTS

UNIT NUMBER: 2

TYPE: $\quad$ PWR

DOCKET NO . : 50-320

COOLING WATER SOURCE : SUSQUEHANNA RIVER

\section{AIRBORNE EFFLUENTS}

NUCLIDES RELEASED

CO-60

$\mathbf{K R}-85$

SR-89

SR-90

NB-95

CS-134

CS-137

UNIDENTIFIED

LIQUID EFFLUENTS

NUCLIDES RELEASED

CS-137

UNIDENTIFIED

TRITIUM

TOTAL AIRBORNE RELEASE

TOTAL LIQUID RELEASE
LICENSEE : METROPOLITAN EDISON \& JERSEY CENTRAL POW LICENSED POWER (MWT):2772.00 INITIAL CRITICALITY:03/28/78

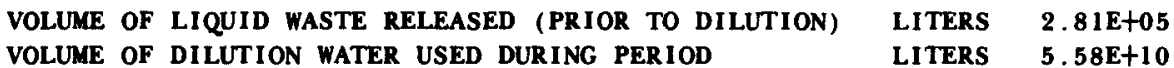

N/A=NOT APPLICABLE

N/D-NOT DETECTED

N/R=NOT REPORTED 
INSTALLATION: THREE MILE ISLAND 2

EFFLUENT AND WASTE DISPOSAL ANNUAL REPORT FOR YEAR 1981 SOLID EFFLUENTS

SOLID WASTE DISPOSITION NUMBER OF SHIPMENTS MODE OF TRANSPORTATION

$\begin{array}{ll}1 & \text { TRACTOR/TRA I LER (VAN) } \\ 2 & \text { TRACTOR/TRAILER (VAN) } \\ 5 & \text { TRACTOR/TRAILER (VAN) }\end{array}$

DESTINATION

BARNWELL SC

RICHLAND WA

WASHINGTON STATE

ESTIMATE OF MAJOR NUCLIDE COMPOS ITION(BY TYPE OF WASTE)

A

CO-58

CO-60

MN-54

B

R-90/Y-90

BA-137M

CS- 134

CS -137

SR-90

Y-90

D

BA- $197 \mathrm{M}$

CS-197

SR-89

SR-90

Y-90

$\begin{array}{lll} & \text { JAN-JUNE } & \text { JULY-DEC } \\ & & \\ \% & & 4.30 E-01 \\ \% & & 8.00 E+01 \\ \% & 1.88 E+01 \\ \% & & 3.50 E-01 \\ & & \\ \% & 4.10 E+01 & 4.39 E+01 \\ \% & 9.00 E+00 & 5.10 E+00 \\ \% & 4.30 E+01 & 4.64 E+01 \\ \% & 3.00 E+00 & 2.32 E+00 \\ \% & 3.00 E+00 & 2.32 E+00 \\ & & \\ \% & & 1.67 E+01 \\ \% & & 1.75 E+01 \\ \% & & 7.95 E+00 \\ \% & & 2.75 E+01 \\ \% & & 2.75 E+01\end{array}$

TYPE OF WASTE

A. SPENT RESINS, FILTER SLUDGES, EVAPORATOR BOTTOMS, ETC.

B. DRY COMPRESS IBLE WASTE, CONTAMINATED EQUI PMENT, ETC

C. IRRADIATED COMPONENTS, CONTROL RODS, ETC.

D. OTHER

$\begin{array}{lc}\text { UNIT } & \text { YEAR TOTAL } \\ \text { M3 } & 4.42 E+00 \\ \text { CI } & 4.00 E-02 \\ \text { MS } & 2.67 E+02 \\ \text { CI } & 4.79 E+01 \\ \text { MS } & \\ \text { CI } & \\ \text { M3 } & 3.16 E+00 \\ \text { CI } & 3.17 E+00\end{array}$

N/A=NOT APPLICABLE

$\mathrm{N} / \mathrm{D}=$ NOT DETECTED

$\mathbf{N} / \mathbf{R}=$ NOT REPORTED 
EFFLUENT AND WASTE DISPOSAL ANNUAL REPORT FOR YEAR 1981

AIRBORNE AND LIQUID EFFLUENTS

UNIT NUMBER : 2

TYPE: $\quad$ PWR

DOCKET NO. : 50-320

COOLING WATER SOURCE : SUSQUEHANNA RIVER

\section{AIRBORNE EFFLUENTS}

NUCLIDES RELEASED

KR-85

ACTIVITY (CI)

CS-134

CS-137

UNIDENTIFIED

TRITIUM

TOTAL AIRBORNE RELEASE

TOTAL LIQUID RELEASE
$8.705-08$
9. $99 \mathrm{E}-07$
1. S3E-06
(CI)
5.59E-02
$0.00 E+00$

LICENSEE : METROPOLITAN EDISON \& JERSEY CENTRAL POWER LICENSED POWER (MWT) :2772.00 INITIAL CRITICALITY : 03/28/78
VOLUME OF LIQUID WASTE RELEASED (PRIOR TO DILUTION) VOLUME OF DILUTION WATER USED DURING PERIOD

N/A=NOT APPLICABLE

N/D=NOT DETECTED

N/R=NOT REPORTED 
INSTALLATION: TMI 2/EPICOR

EFFLUENT AND WASTE DISPOSAL ANNUAL REPORT FOR YEAR 1981 SOLID EFFLUENTS

SOLID WASTE DISPOSITION NUMBER OF SHIPMENTS MODE OF TRANSPORTATION 22 SHIPPING CASK/TRUCK TRACTOR/FLATBED TRAILER

TRACTOR/TRAILER (CASK)

DESTINATION

WASHINGTON STATE

WASHINGTON STATE

23

RICHLAND WA

ESTIMATE OF MAJOR NUCLIDE COMPOSITION(BY TYPE OF WASTE)

$A$

BA-137M

CS-134

CS -137

SB- 125

SR-90

Y-90

JAN-JUNE JULY-DEC
$3.35 \mathrm{E}+01$
7. 47E+00
4. $60 \mathrm{E}+00$
$3.54 \mathrm{E}+01 \quad 6.61 \mathrm{E}+00$
9.90E+00
$1.85 \mathrm{E}+01$
9. 90E+00
2.94E+0
2.94E+01

TYPE OF WASTE

A. SPENT RESINS, FILTER SLUDGES, EVAPORATOR BOTTOMS, ETC.

B. DRY COMPRESSIBLE WASTE, CONTAMINATED EQUI PMENT, ETC.

C. IRRADIATED COMPONENTS, CONTROL RODS, ETC.

D. OTHER

UNIT YEAR TOTAL

M3 1.51E+02

CI $3.50 \mathrm{E}+02$

M3

CI

M3

CI

M3

CI

N/A=NOT APPLICABLE

N/D $=$ NOT DETECTED

N/R=NOT REPORTED 
EFFLUENT AND WASTE DISPOSAL ANNUAL REPORT FOR YEAR 1981 AIRBORNE AND LIQUID EFFLUENTS

UNIT NUMBER: 1

TYPE: $\quad$ PWR

DOCKET NO. : 50-344

COOLING WATER SOURCE: COLUMBIA RIVER

\section{AIRBORNE EFFLUENTS}

NUCLIDES RELEASED

AR-41

MN-54

CO-57

$\mathrm{CO}-58$

C0-60

KR-85

KR-85M

KR-87

KR-88

SR-89

SR-90

NB-95

ZR-95

RU- 103

1-131

$\mathrm{XE}-131 \mathrm{M}$

I-132

I-133

XE-1 133

XE-133M

CS-134

I-134

I-135

XE-135

XE-135M

CS-137

XE-137

XE- 138

BA-140

LA-140

CE-144

UNIDENTIFIED

LIQUID EFFLUENTS

NUCLIDES RELEASED

F-1 8

NA-24

CR-51

MN-54

CO-57

CO-58

FE-59

CO-60

$\mathrm{ZN}-65$
LICENSEE : PORTLAND GENERAL ELECTR IC

LICENSED POWER (MWT) : $\mathbf{3 4 1 1 . 0 0}$

INITIAL CRITICALITY : $12 / 15 / 75$

N/A=NOT APPLICABLE N/D =NOT DETECTED N/R=NOT REPORTED

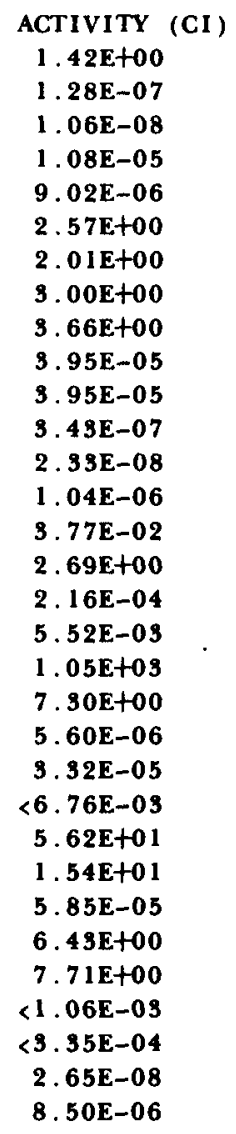


EFFLUENT AND WASTE DISPOSAL ANNUAL REPORT FOR YEAR 1981 AIRBORNE AND LIQUID EFFLUENTS

\begin{tabular}{|c|c|c|c|}
\hline \multicolumn{4}{|l|}{ LIQUID EFFLUENTS } \\
\hline NUCL IDES RELEASED & \multicolumn{3}{|l|}{ ACTIVITY (CI) } \\
\hline KR-85M & \multicolumn{3}{|l|}{$6.34 \mathrm{E}-06$} \\
\hline SR-89 & \multicolumn{3}{|l|}{$<8.29 \mathrm{E}-03$} \\
\hline SR-90 & \multicolumn{3}{|l|}{$<1.67 \mathrm{E}-03$} \\
\hline NB-95 & \multicolumn{3}{|l|}{$2.01 \mathrm{E}-02$} \\
\hline ZR-95 & \multicolumn{3}{|l|}{$1.05 E-02$} \\
\hline м0-99 & \multicolumn{3}{|l|}{ 3.34E-04 } \\
\hline TC-99M & \multicolumn{3}{|l|}{$2.94 E-04$} \\
\hline RU- 103 & \multicolumn{3}{|l|}{$6.34 \mathrm{E}-03$} \\
\hline$A G-110 \mathrm{M}$ & \multicolumn{3}{|l|}{$1.53 \mathrm{E}-03$} \\
\hline SN-113 & \multicolumn{3}{|l|}{$4.13 E-04$} \\
\hline SB-124 & \multicolumn{3}{|l|}{ 1. 14E-02 } \\
\hline$S B-125$ & \multicolumn{3}{|l|}{$6.83 E-02$} \\
\hline $1-191$ & \multicolumn{3}{|l|}{$<5.69 \mathrm{E}-02$} \\
\hline $\mathrm{XE}-131 \mathrm{M}$ & \multicolumn{3}{|l|}{$3.53 \mathrm{E}-03$} \\
\hline$I-132$ & \multicolumn{3}{|l|}{$7.16 \mathrm{E}-05$} \\
\hline TE-132 & \multicolumn{3}{|l|}{$3.63 E-04$} \\
\hline I-133 & \multicolumn{3}{|l|}{$1.24 E-02$} \\
\hline$X E-133$ & \multicolumn{3}{|l|}{$6.93 \mathrm{E}-01$} \\
\hline$X E-133 M$ & \multicolumn{3}{|l|}{$7.85 \mathrm{E}-04$} \\
\hline CS-134 & \multicolumn{3}{|l|}{$6.30 \mathrm{E}-02$} \\
\hline$I-135$ & \multicolumn{3}{|l|}{$2.15 E-03$} \\
\hline$X E-135$ & \multicolumn{3}{|l|}{ 1. $59 \mathrm{E}+00$} \\
\hline$X E-135 M$ & \multicolumn{3}{|l|}{$1.02 E+00$} \\
\hline CS 136 & \multicolumn{3}{|l|}{$1.96 \mathrm{E}-03$} \\
\hline CS-137 & \multicolumn{3}{|l|}{$9.08 E-02$} \\
\hline BA- 140 & \multicolumn{3}{|l|}{$<6.99 \mathrm{E}-02$} \\
\hline LA- 140 & $<1.65 \mathrm{E}-02$ & & \\
\hline CE-141 & $4.36 E-04$ & & \\
\hline CE-144 & $9.14 E-03$ & & \\
\hline NP-239 & 1. $89 \mathrm{E}-03$ & & \\
\hline UNI DENT IF IED & $7.62 \mathrm{E}-04$ & & \\
\hline TRITIUM & (CI) & & \\
\hline TOTAL AIRBORNE RELEASE & $4.03 E+01$ & & \\
\hline TOTAL LIQUID RELEASE & $1.03 E+02$ & & \\
\hline VOLUME OF LIQUID WASTE $R$ & ED (PRIOR TO DILUTION) & LITERS & $5.06 E+07$ \\
\hline VOLUME OF DILUTION WATER & D DURING PERIOD & LITERS & $4.35 E+10$ \\
\hline
\end{tabular}

LITERS $4.35 E+10$

N/A=NOT APPLICABLE

N/D=NOT DETECTED

N/R=NOT REPORTED 
INSTALLATION : TROJAN

EFFLUENT AND WASTE DISPOSAL ANNUAL REPORT FOR YEAR 1981 SOLID EFFLUENTS

SOLID WASTE DISPOSITION NUMBER OF SHI PMENTS MODE OF TRANSPORTATION

TRUCK

DESTINATION 27

RICHLAND WA

IRRADI ATED FUEL SHI PMENTS (DISPOSITION)

NUMBER OF SHI PMENTS MODE OF TRANSPORTATION

DESTINATION

ESTIMATE OF MAJOR NUCLIDE COMPOSITION(BY TYPE OF WASTE)

A

BA- 140

CE- 144

CO-57

CO-58

CO-60

CR-5 1

CS-134

CS- 136

CS-137

FE-55

FE-59

I-131

I - 193

LA- 140

MN-54

NB-95

NI -69

RU-103

SB-1 25

SR-90

ZR-95

B

CE- 141

CE-1 44

CO-58

CO-60

CR-5 1

CS-134

CS-1 36

CS-137

FE-59

I -131

MN-54

NB-95

RU- 103

ZN-65

ZR-95

JAN-JUNE JULY-DEC

\begin{tabular}{|c|c|c|}
\hline$\%$ & $1.98 E-03$ & \\
\hline$\%$ & $9.48 E-01$ & 3.09E-01 \\
\hline$\%$ & $1.99 \mathrm{E}-01$ & 5. 21 E-02 \\
\hline$\%$ & 1. $32 E+01$ & $5.55 \mathrm{E}-01$ \\
\hline$\%$ & $6.56 E+01$ & $3.74 \mathrm{E}+01$ \\
\hline$\%$ & $1.03 \mathrm{E}+00$ & $4.68 \mathrm{E}-03$ \\
\hline$\%$ & $5.31 \mathrm{E}+00$ & 4. $16 \mathrm{E}+00$ \\
\hline$\%$ & $2.97 \mathrm{E}-03$ & \\
\hline$\%$ & 1. $19 \mathrm{E}+01$ & 1. $02 \mathrm{E}+01$ \\
\hline$\%$ & & 3. $19 \mathrm{E}+01$ \\
\hline$\%$ & $4.35 \mathrm{E}-02$ & \\
\hline$\%$ & $2.47 \mathrm{E}-02$ & \\
\hline$\%$ & $9.89 E-04$ & \\
\hline$\%$ & $9.89 E-04$ & \\
\hline$\%$ & $1.14 \mathrm{E}+00$ & $2.30 \mathrm{E}+00$ \\
\hline$\%$ & $2.93 \mathrm{E}-01$ & 3. 19E-03 \\
\hline$\%$ & & $1.28 \mathrm{E}+01$ \\
\hline$\%$ & $8.51 \mathrm{E}-02$ & \\
\hline$\%$ & $8.91 \mathrm{E}-03$ & $2.06 \mathrm{E}-02$ \\
\hline$\%$ & & $2.61 \mathrm{E}-01$ \\
\hline$\%$ & $1.76 \mathrm{E}-01$ & $4.68 E-03$ \\
\hline$\%$ & $1.04 \mathrm{E}-01$ & 1. 22E-01 \\
\hline$\%$ & $2.16 E+01$ & 3. $28 \mathrm{E}+00$ \\
\hline$\%$ & $2.45 \mathrm{E}+01$ & $2.52 \mathrm{E}+01$ \\
\hline$\%$ & $1.86 \mathrm{E}+01$ & $2.90 E+01$ \\
\hline$\%$ & $1.79 E+01$ & 1. $30 \mathrm{E}+01$ \\
\hline * & $1.11 \mathrm{E}+00$ & $5.59 \mathrm{E}+00$ \\
\hline$\%$ & 3. 48E-02 & $1.22 \mathrm{E}-01$ \\
\hline$\%$ & 3. I $7 E+00$ & $7.78 \mathrm{E}+00$ \\
\hline$\%$ & 5.57E-01 & $3.65 \mathrm{E}-01$ \\
\hline$\%$ & $5.57 \mathrm{E}-01$ & 1. $70 \mathrm{E}+00$ \\
\hline$\%$ & $3.17 \mathrm{E}+00$ & 3. $40 \mathrm{E}+00$ \\
\hline$\%$ & $5.22 E+00$ & $5.71 \mathrm{E}+00$ \\
\hline$\%$ & $6.96 \mathrm{E}-01$ & $8.51 \mathrm{E}-01$ \\
\hline 8 & & 1. $22 \mathrm{E}-01$ \\
\hline$\%$ & $2.68 \mathrm{E}+00$ & 3. $65 \mathrm{E}+00$ \\
\hline
\end{tabular}

N/A $=$ NOT APPLICABLE

$N / D=N O T$ DETECTED

$\mathbf{N} / \mathbf{R}=\mathbf{N O T}$ REPORTED 
INSTALLATION : TROJAN

EFFLUENT AND WASTE DISPOSAL ANNUAL REPORT FOR YEAR 1981 SOLID EFFLUENTS

ESTIMATE OF MAJOR NUCLIDE COMPOSITION(BY TYPE OF WASTE)

JAN-JUNE JULY-DEC

TYPE OF WASTE

A. SPENT RESINS, FILTER SLUDGES, EVAPORATOR BOTTOMS , ETC.

B. DRY COMPRESS IBLE WASTE, CONTAMINATED

EQUI PMENT, ETC.

C. IRRADIATED COMPONENTS, CONTROL.

RODS, ETC.

D. OTHER

UNIT YEAR TOTAI

M3 $\quad 1.49 \mathrm{E}+02$

CI $\quad 1.04 \mathrm{E}+03$

M3 $2.26 \mathrm{E}+02$

CI 3.70E+00

M3

CI

M9

CI

$N / A=$ NOT APPLICABLE

$N / D=N O T$ DETECTED

N/R $=$ NOT REPORTED 
EFFLUENT AND WASTE DISPOSAL ANNUAL REPORT FOR YEAR 1981

AIRBORNE AND LIQUID EFFLUENTS

UNIT NUMBER: 3

TYPE : PWR

DOCKET NO. : 50-250

COOLING WATER SOURCE : CLOSED CANAL SYSTEM UNIT NUMBER : 4

TYPE : PWR

DOCKET NO . : 50-251

COOLING WATER SOURCE:CLOSED CANAL SYSTEM

AIRBORNE EFFLUENTS

NUCLIDES RELEASED

AR-41

CR-5 1

$\mathrm{MN}-54$

CO-57

CO-58

CO-60

BR-82

$\mathrm{KR}-85$

KR-85M

KR-87

KR-88

SR-89

SR-90

NB-95

RU- 103

SB-125

$1-131$

XE-131

$X E-131 M$

I -133

$\mathrm{XE}-139$

$X E-133 M$

CS-134

I-135

$\mathrm{XE}-135$

$\mathrm{XE}-135 \mathrm{M}$

CS-136

CS -137

BA-140

LA- 140

LIQUID EFFLUENTS

NUCLIDES RELEASED

F-18

NA-24

CR-51

MN-54

CO-57

CO-58

FE-59

N/A=NOT APPLICABLE

$N / D=N O T$ DETECTED

N/R=NOT REPORTED

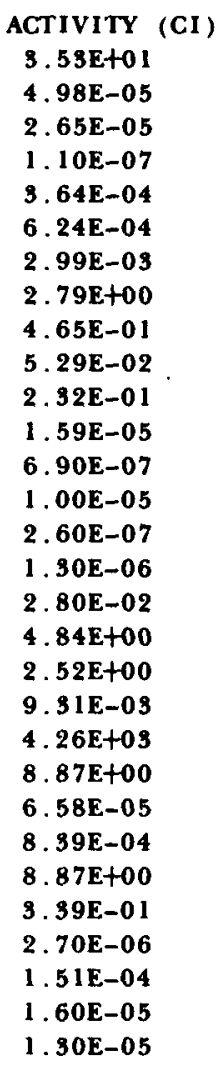

1. 30E-03
LICENSEE : FLORIDA POWER \& LIGHT LICENSED POWER (MWT) $: 2200.00$ INITIAL CRITICALITY : $10 / 20 / 72$

LICENSEE ; FLOR IDA POWER \& LIGHT LICENSED POWER (MWT) :2200.00 INITIAL CRITICALITY:06/11/73 
EFFLUENT AND WASTE DISPOSAL ANNUAL REPORT FOR YEAR 1981

AIRBORNE AND LIQUID EFFLUENTS

$\begin{array}{lc}\text { LIQUID EFFLUENTS } & \\ \text { NUCLIDES RELEASED } & \text { ACTIVITY (CI) } \\ \text { CO-60 } & 6.17 E-02 \\ \text { ZN-65 } & 2.38 E-04 \\ \text { SR-89 } & 3.39 E-03 \\ \text { SR-90 } & 9.49 E-05 \\ \text { NB-95 } & 3.97 E-03 \\ \text { ZR-95 } & 1.69 E-03 \\ \text { ZR-97 } & 1.01 E-03 \\ \text { RU-103 } & 4.01 E-05 \\ \text { AG-110M } & 2.03 E-03 \\ \text { SB-124 } & 4.61 E-03 \\ \text { SB-125 } & 1.13 E-02 \\ \text { I-131 } & 1.63 E-02 \\ \text { I-132 } & 3.28 E-03 \\ \text { I-133 } & 1.54 E-02 \\ \text { CS-134 } & 2.95 E-02 \\ \text { I-134 } & 6.70 E-04 \\ \text { I-135 } & 1.48 E-03 \\ \text { CS-136 } & 1.03 E-04 \\ \text { CS-137 } & 4.18 E-02 \\ \text { LA-140 } & 2.66 E-04 \\ \text { CE-144 } & 1.52 E-04 \\ & \\ \text { TRITIUM } & \\ \text { TOTAL AIRBORNE RELEASE } & 6.86 E-01 \\ \text { TOTAL LIQUID RELEASE } & 1.95 E+02 \\ & \end{array}$

VOLUME OF LIQUID WASTE RELEASED (PRIOR TO DILUTION) LITERS 2.04E+08 VOLUME OF DILUTION WATER USED DURING PERIOD 
INSTALLATION:TURKEY POINT

EFFLUENT AND WASTE DISPOSAL ANNUAL REPORT FOR YEAR 1981 SOLID EFFLUENTS

SOLID WASTE DISPOSITION

NULBER OF SHIPMENTS MODE OF TRANSPORTATION

DESTINATION

55

BARNWELL SC

27

RICHLAND WA

TYPE OF WASTE

A. SPENT RESINS, FILTER SLUDGES, EVAPORATOR BOTTOMS, ETC.

B. DRY COMPRESSIBLE WASTE, CONTAMINATED

EQUI PMENT, ETC.

C. IRRADIATED COMPONENTS, CONTROL

RODS, ETC

UNIT YEAR TOTAL

M3 1.25E+03

CI 1.17E+02

D. OTHER

M9

CI

Mg

CI

M3

CI

N/A=NOT APPLICABLE

N/D=NOT DETECTED

N/R=NOT REPORTED 
EFFLUENT AND WASTE DISPOSAL ANNUAL REPORT FOR YEAR 1981 AIRBORNE AND LIQUID EFFLUENTS

\begin{tabular}{|c|c|}
\hline \multicolumn{2}{|l|}{ UNIT NUMBER: 1} \\
\hline \multicolumn{2}{|l|}{ TYPE : $\quad$ BWR } \\
\hline \multicolumn{2}{|l|}{ DOCKET NO.: :50-271 } \\
\hline \multicolumn{2}{|c|}{ COOLING WATER SOURCE : CONNECTICUT RIVER } \\
\hline \multicolumn{2}{|l|}{ AIRBORNE EFFLUENTS } \\
\hline NUCLIDES RELEASED & ACTIVITY (CI) \\
\hline MN-54 & $<2.96 \mathrm{E}-04$ \\
\hline CO-58 & $5.66 \mathrm{E}-05$ \\
\hline $\mathrm{CO}-60$ & 1. $60 \mathrm{E}-03$ \\
\hline $\mathrm{ZN}-65$ & $<5.52 \mathrm{E}-04$ \\
\hline KR-85 & N/D \\
\hline $\mathbf{K R}-\mathbf{8 5 M}$ & $<2.86 \mathrm{E}+01$ \\
\hline $\mathbf{K R}-\mathbf{8 7}$ & $<8.35 \mathrm{E}+01$ \\
\hline KR-88 & $<7.40 \mathrm{E}+01$ \\
\hline SR-89 & $5.79 \mathrm{E}-05$ \\
\hline SR-90 & 3. 19E-06 \\
\hline I-131 & $1.63 E-03$ \\
\hline I - 133 & $<6.22 \mathrm{E}-03$ \\
\hline $\mathrm{XE}-133$ & $<4.68 \mathrm{E}+02$ \\
\hline CS-134 & $<1.84 \mathrm{E}-04$ \\
\hline$I-135$ & $<3.29 E-02$ \\
\hline $\mathrm{XE}-195$ & $<1.48 \mathrm{E}+02$ \\
\hline$X E-195 M$ & $<5.18 \mathrm{E}+02$ \\
\hline CS-137 & $6.60 \mathrm{E}-04$ \\
\hline $\mathrm{XE}-138$ & $<1.86 \mathrm{E}+03$ \\
\hline BA-LA-140 & $<8.44 \mathrm{E}-04$ \\
\hline \multicolumn{2}{|l|}{ LIQUID EFFLUENTS } \\
\hline NUCLIDES RELEASED & ACTIVITY (CI) \\
\hline NA-24 & 3. $79 \mathrm{E}-04$ \\
\hline CR-51 & N/D \\
\hline MN-54 & N/D \\
\hline $\mathrm{CO}-58$ & N/D \\
\hline FE-59 & N/D \\
\hline $\mathrm{CO}-60$ & $2.13 \mathrm{E}-03$ \\
\hline $\mathrm{ZN}-65$ & $6.54 E-04$ \\
\hline SR-89 & $1.41 \mathrm{E}-05$ \\
\hline SR-90 & $1.35 E-06$ \\
\hline ZR-NB-95 & N/D \\
\hline MO-99 & N/D \\
\hline TC-99M & N/D \\
\hline $\mid-131$ & N/D \\
\hline $\mathrm{XE}-133$ & N/D \\
\hline CS-134 & $1.58 \mathrm{E}-03$ \\
\hline$X E-135$ & $9.65 \mathrm{E}-05$ \\
\hline CS-137 & $5.48 \mathrm{E}-03$ \\
\hline BA-LA- 140 & N/D \\
\hline CE-141 & N/D \\
\hline
\end{tabular}

LICENSEE : VERMONT YANKEE NUCLEAR POWER LICENSED POWER (MWT) $: 1593.00$ INITIAL CRITICALITY:03/24/72

N/A=NOT APPLICABLE $N / D=N O T$ DETECTED N/R=NOT REPORTED 
EFFLUENT AND WASTE DISPOSAL ANNUAL REPORT FOR YEAR 1981 AIRBORNE AND LIQUID EFFLUENTS

TRITIUM

TOTAL AIRBORNE RELEASE

TOTAL LIQUID RELEASE
(CI)

1. $96 \mathrm{E}+01$

3. 70E-01
VOLUME OF LIQUID WASTE RELEASED (PRIOR TO DILUTION) VOLUME OF DILUTION WATER USED DURING PERIOD
LITERS

LITERS
$6.74 \mathrm{E}+04$

1. $11 \mathrm{E}+08$

N/A =NOT APPLICABLE

N/D $=$ NOT DETECTED

N/R=NOT REPORTED 
INSTALLATION : VERMONT YANKEE

EFFLUENT AND WASTE DISPOSAL ANNUAL REPORT FOR YEAR 1981 SOLID EFFLUENTS

SOLID WASTE DISPOSITION NUMBER OF SHIPMENTS

$\begin{array}{rr}36 & \text { TRUCK } \\ 5 & \text { TRUCK } \\ 3 & \text { TRUCK }\end{array}$

DESTINATION

BARNWELL, S.C.

BEATTY, NV

RICHLAND, WA

IRRADIATED FUEL SHI PMENTS (DISPOS ITION)

NUMBER OF SHIPMENTS MODE OF TRANSPORTATION

$\mathbf{0}$

DESTINATION

ESTIMATE OF MAJOR NUCLIDE COMPOSITION(BY TYPE OF WASTE)

A

BA- 140

CO-58

C0-60

CS- 134

CS-137

I-131

MN-54

SB- 124

$\mathrm{ZN}-65$

ZR-95

B

C0-58

C0-60

CS-134

CS-137

MN-54

$\mathrm{ZN}-65$

C

Co-58

C0-60

CS-134

CS-137

MN-54

ZN-65

\begin{tabular}{|c|c|c|}
\hline & J AN-J UNE & JULY-DEC \\
\hline$\%$ & $6.70 \mathrm{E}-0 \mathrm{~S}$ & \\
\hline$\%$ & $1.90 \mathrm{E}+00$ & $2.00 \mathrm{E}+00$ \\
\hline$\%$ & $1.60 \mathrm{E}+01$ & $1.60 \mathrm{E}+01$ \\
\hline$x$ & 1. $.90 \mathrm{E}+01$ & 2.00E+01 \\
\hline$\%$ & $5.30 E+01$ & 5.30E+01 \\
\hline$x$ & $1.30 \mathrm{E}-01$ & \\
\hline$\%$ & $2.10 E+00$ & $2.00 E+00$ \\
\hline$\%$ & $2.40 E-01$ & \\
\hline$\%$ & $7.30 E+00$ & $7.00 \mathrm{E}+00$ \\
\hline$x$ & 3.20E-01 & \\
\hline$\%$ & $9.80 \mathrm{E}-01$ & $9.80 \mathrm{E}-01$ \\
\hline ж & $2.40 E+01$ & $2.40 \mathrm{E}+01$ \\
\hline$\%$ & $2.30 \mathrm{E}+01$ & $2.30 \mathrm{E}+01$ \\
\hline$\%$ & $4.10 E+01$ & $4.10 \mathrm{E}+01$ \\
\hline \% & $2.50 \mathrm{E}+00$ & 2.50E+00 \\
\hline$\%$ & $8.40 E+00$ & $8.40 \mathrm{E}+00$ \\
\hline$\%$ & & $9.80 \mathrm{E}-01$ \\
\hline$\%$ & & $2.40 \mathrm{E}+01$ \\
\hline$\%$ & & $2.30 \mathrm{E}+01$ \\
\hline$\%$ & & $4.10 \mathrm{E}+01$ \\
\hline$\%$ & & 2.50E+00 \\
\hline$\%$ & & $8.40 E+00$ \\
\hline
\end{tabular}

TYPE OF WASTE

A. SPENT RESINS, FILTER SLUDGES, EVAPORATOR

UNIT YEAR TOTAL BOTTOMS, ETC

B. DRY COMPRESS I BLE WASTE, CONTAMINATED EQUI PMENT, ETC.

C. IRRADIATED COMPONENTS, CONTROL RODS , ETC

D. OTHER

$\begin{array}{ll}\text { M3 } & 1.72 E+02 \\ \text { CI } & 1.02 E+03 \\ \text { M3 } & 2.65 E+02 \\ \text { CI } & 8.03 E+00 \\ \text { M3 } & 1.20 E+00 \\ \text { CI } & 7.90 E+01 \\ \text { M3 } & \\ \text { CI } & \end{array}$

N/A $=$ NOT APPLICABLE

$N / D=N O T$ DETECTED

N/R $=$ NOT REPORTED 
EFFLUENT AND WASTE DISPOSAL ANNUAL REPORT FOR YEAR 1981 AIRBORNE AND LIQUID EFFLUENTS

UNIT NUMBER : 1

TYPE: $\quad$ PWR

DOCKET NO . : 50-29

COOLING WATER SOURCE: DEERFIELD RIVER

\section{AIRBORNE EFFLUENTS \\ NUCLIDES RELEASED}

$\mathrm{H}-3$

C- 14

AR-37

AR-41

CR-5 1

MN-54

CO-58

FE-59

C0-60

KR-85

KR-85M

KR-87

KR-88

SR-89

SR-90

ZR-NB-95

AG- $110 \mathrm{M}$

SB-1 24

SB-1 25

$|-13|$

XE-13 IM

I-133

XE-1 33

XE-133M

CS-134

I-135

XE-1 35

XE-195M

CS-137

XE-1 38

BA-LA- 140

CE- 141

CE-144

LIQUID EFFLUENTS

NUCLIDES RELEASED

H-3

C- 14

CR-51

MN-54

CO-58

FE-59

CO-60

ZN-65
LICENSEE : YANKEE ATOMIC ELECTRIC

LICENSED POWER (MWT) : 600.00

INITIAL CRITICALITY : 08/19/60

N/A=NOT APPLICABLE

$N / D=N O T$ DETECTED
$N / R=N O T$ REPORTED

$N / D=$ NOT DETECTED
$N / R=$ NOT REPORTED

$$
\begin{gathered}
\text { ACTIVITY (CI) } \\
3.07 E+00 \\
4.71 E-01 \\
5.96 E-01 \\
1.33 E+00 \\
<1.54 E-05 \\
9.61 E-06 \\
<8.90 E-06 \\
<3.28 E-06 \\
<1.10 E-05 \\
6.53 E+00 \\
1.44 E+00 \\
1.52 E+00 \\
2.48 E+00 \\
<1.50 E-06 \\
<1.11 E-06 \\
<3.41 E-06 \\
<3.14 E-06 \\
<3.12 E-06 \\
<1.18 E-05 \\
1.68 E-04 \\
1.18 E+00 \\
<5.76 E-05 \\
8.03 E+01 \\
<1.19 E+00 \\
<1.93 E-06 \\
<1.09 E-04 \\
2.90 E+01 \\
4.55 E+01 \\
<3.18 E-06 \\
1.40 E+00 \\
<7.25 E-06 \\
<2.63 E-06 \\
<1.23 E-05 \\
\text { ACTIVITY }(C I) \\
1.03 E+02 \\
5.25 E-03 \\
<1.64 E-04 \\
3.44 E-04 \\
2.09 E-04 \\
4.60 E-05 \\
4.14 E-04 \\
<7.40 E-05 \\
\end{gathered}
$$


EFFLUENT AND WASTE DISPOSAL ANNUAL REPORT FOR YEAR 1981 AIRBORNE AND LIQUID EFFLUENTS

$\begin{array}{lc}\text { LIQUID EFFLUENTS } & \\ \text { NUCLIDES RELEASED } & \text { ACTIVITY (CI) } \\ \text { SE-75 } & <3.57 E-05 \\ \text { SR-89 } & 3.19 E-04 \\ \text { SR-90 } & 1.88 E-05 \\ \text { ZR-NB-95 } & <.86 E-05 \\ \text { MO-99 } & <1.68 E-04 \\ \text { TC-99M } & <1.93 E-05 \\ \text { RU-103 } & <1.40 E-05 \\ \text { RU-106 } & <1.93 E-05 \\ \text { AG-11OM } & <4.91 E-05 \\ \text { SB-124 } & <3.00 E-05 \\ \text { SB-125 } & 6.71 E-06 \\ \text { I-131 } & 3.32 E-03 \\ \text { TE-132 } & 8.15 E-06 \\ \text { I-193 } & 4.07 E-04 \\ \text { XE-133 } & 9.01 E-01 \\ \text { CS-134 } & 1.14 E-03 \\ \text { XE-135 } & <4.10 E-03 \\ \text { CS-137 } & 2.47 E-03 \\ \text { BA-LA-140 } & <1.65 E-04 \\ \text { CE-141 } & <1.10 E-05 \\ \text { CE-144 } & <7.80 E-05 \\ \text { UNIDENTIFIED } & <9.62 E-05 \\ & \\ \text { TRITIUM } & \\ \text { TOTAL AIRBORNE RELEASE } & 3.07 E+00 \\ \text { TOTAL LIQUID RELEASE } & 1.03 E+02 \\ & \\ \text { VOLUME OF LIQUID WASTE RELEASED (PRIOR TO DILUTION) } \\ \text { VOLUME OF DILUTION WATER USED DURING PERIOD } \\ \end{array}$

N/A $=$ NOT APPLICABLE

$N / D=N O T$ DETECTED

N/R=NOT REPORTED 
INSTALLATION: YANKEE ROWE

EFFLUENT AND WASTE DISPOSAL ANNUAL REPORT FOR YEAR 1981 SOLID EFFLUENTS

SOLID WASTE DISPOSITION

NUMBER OF SHI PMENTS MODE OF TRANSPORTATION

23

TRUCK

TRUCK

DESTINATION

BARNWELL, S.C.

RICHLAND, WA

IRRADIATED FUEL SHI PMENTS (DISPOS ITION)

NUMBER OF SHIPMENTS MODE OF TRANSPORTATION

DEST LNATION

TYPE OF WASTE

A. SPENT RESINS, FILTER SLUDGES, EVAPORATOR BOTTOMS, ETC.

B. DRY COMPRESS IBLE WASTE, CONTAMINATED

EQUI PMENT, ETC

C. IRRADIATED COMPONENTS, CONTROL

RODS, ETC.

D. OTHER

UNIT YEAR TOTAL

M3 T.95E+01

CI $\quad 5.47 \mathrm{E}+00$

M3 $2.49 E+02$

CI 6.24E+01

M3 $0.00 E+00$

CI $\quad 0.00 E+00$

M3 $0.00 \mathrm{E}+00$

CI $\quad 0.00 E+00$

N/A=NOT APPLICABLE

N/D=NOT DETECTED

N/R=NOT REPORTED 
EFFLUENT AND WASTE DISPOSAL ANNUAL REPORT FOR YEAR 1981 AIRBORNE AND LIQUID EFFLUENTS

UNIT NUMBER : 1

TYPE : PWR

DOCKET NO. : 50-295

COOLING WATER SOURCE: LAKE MICHIGAN

UNIT NUMBER: 2

TYPE :

PWR

DOCKET NO . : 50-304

COOLING WATER SOURCE:LAKE MICHIGAN

\section{AIRBORNE EFFLUENTS}

NUCLIDES RELEASED

AR-41

CO-58

CO-60

KR-85

KR-85M

KR-87

KR-88

SR-89

SR-90

NB-95

I-131

XE-131M

1-132

I-133

XE-133

$\mathrm{XE}-133 \mathrm{M}$

CS-134

I-134

I-135

$\mathrm{XE}-135$

$\mathrm{XE}-135 \mathrm{M}$

CS-137

CS-138

$\mathrm{XE}-138$

TRITIUM

TOTAL AIRBORNE RELEASE
LICENSEE : COMMONWEALTH EDISON LICENSED POWER (MWT) : $\mathbf{3 2 5 0 . 0 0}$ INITIAL CRITICALITY: 06/19/73

LICENSEE : COMMONWEALTH EDISON LICENSED POWER (MWT) :3250.00 INITIAL CRITICALITY: $12 / 24 / 73$
ACTIVITY (CI)

2. 69E+0 1

1. 08E-03

3. 33E-04

9. 42E-01

$1.70 \mathrm{E}+01$

9.31E+00

3. $05 \mathrm{E}+01$

1. $31 \mathrm{E}-04$

1. 86E-05

1. $05 \mathrm{E}-04$

5. 15E-03

4. $09 \mathrm{E}+00$

1. 23E-05

9. 22E-04

$6.37 \mathrm{E}+03$

$7.60 \mathrm{E}+00$

1. $56 \mathrm{E}-08^{\circ}$

$4.66 \mathrm{E}-06$

6. 58E-04

2. $92 \mathrm{E}+02$

2. $64 \mathrm{E}+01$

2. 45E-05

1. $53 \mathrm{E}-05$

1. 14E+00

(CI)

N/A $=$ NOT APPLICABLE

$N / D=N O T$ DETECTED

N/R=NOT REPORTED 
EFFLUENT AND WASTE DISPOSAL ANNUAL REPORT FOR YEAR 1981 AIRBORNE AND LIQUID EFFLUENTS

LIQUID EFFLUENTS
NUCL IDES RELEASED
CR-5 1
MN-54
CO-58
FE-59
CO-60
KR-85
SR-89
SR-90
SR-92
NB-95
ZR-95
AG-110M
SB-124
I-131
XE-133
XE-133M
CS-134
XE-135
CS-136
CS-137
BA-LA-140
W-187
TOTAL LIQUID RELEAS

VOLUME OF LIQUID WASTE RELEASED (PRIOR TO DILUTION) LITERS 1.14E+08 VOLUME OF DILUTION WATER USED DURING PERIOD 
EFFLUENT AND WASTE DISPOSAL ANNUAL REPORT FOR YEAR 1981 AIRBORNE AND LIQUID EFFLUENTS

\begin{tabular}{l|l} 
LIQUID EFFLUENTS & \\
NUCLIDES RELEASED & ACTIVITY (CI) \\
CR-5 I & $1.48 E-01$ \\
MN-54 & $1.43 E-03$ \\
CO-58 & $3.48 E-01$ \\
FE-59 & $5.05 E-04$ \\
CO-60 & $2.66 E-01$ \\
KR-85 & $4.20 E-01$ \\
SR-92 & $8.04 E-03$ \\
NB-95 & $3.17 E-02$ \\
ZR-95 & $8.54 E-03$ \\
AG-110M & $7.44 E-02$ \\
SB-124 & $4.03 E-02$ \\
I-131 & $1.82 E-03$ \\
I-133 & $1.92 E-04$ \\
XE-133 & $1.66 E+00$ \\
XE-133M & $1.82 E-03$ \\
CS-134 & $9.93 E-03$ \\
XE-135 & $2.99 E-03$ \\
CS-136 & $1.15 E-03$ \\
CS-137 & $3.96 E-02$ \\
BA-LA-140 & $1.32 E-04$ \\
CE-144 & $9.27 E-04$ \\
W-187 & $4.64 E-02$ \\
TOTAL LIQUID RELEASE & $2.66 E+02$ \\
& \\
VOLUME OF LIQUID WASTE RELEASED (PRIOR TO DILUTION) \\
VOLUME OF DILUTION WATER USED DURING PERIOD \\
& \\
\hline
\end{tabular}

N/A=NOT APPLICABLE

N/D $=$ NOT DETECTED

N/R=NOT REPORTED 
EFFLUENT AND WASTE DISPOSAL ANNUAL REPORT FOR YEAR 1981 SOLID EFFLUENTS

\section{SOLID WASTE DISPOSITION \\ NUMBER OF SHIPMENTS MODE OF TRANSPORTATION 167}

DESTINATION

TYPE OF WASTE

A. SPENT RESINS, FILTER SLUDGES, EVAPORATOR

UNIT YEAR TOTAL BOTTOMS, ETC.

B . DRY COMPRESS IBLE WASTE, CONTAMINATED

1. 53E+03

EQU I PMENT, ETC.

CI $3.44 E+03$

C. IRRADIATED COMPONENTS, CONTROL

RODS, ETC

D. OTHER

M3

CI

M3

CI

MS

CI

$N / A=$ NOT APPLICABLE

$N / D=N O T$ DETECTED

$N / R=$ NOT REPORTED 